\title{
Thermal Hydraulic Characteristics Study of Prototype NET and CEA Cable-in-Conduit Conductors (CICCs)
}

by

Ryuji Maekawa

A dissertation submitted in partial fulfillment of the requirements for the degree of

Doctor of Philosophy

(Nuclear Engineering and Engineering Physics)

at the

UNIVERSITY OF WISCONSIN-MADISON

1995 


\section{DISCLAIMER}

This report was prepared as an account of work sponsored by an agency of the United States Government. Neither the United States Government nor any agency thereof, nor any of their employees, make any warranty, express or implied, or assumes any legal liability or responsibility for the accuracy, completeness, or usefulness of any information, apparatus, product, or process disclosed, or represents that its use would not infringe privately owned rights. Reference herein to any specific commercial product, process, or service by trade name, trademark, manufacturer, or otherwise does not necessarily constitute or imply its endorsement, recommendation, or favoring by the United States Government or any agency thereof. The views and opinions of authors expressed herein do not necessarily state or reflect those of the United States Government or any agency thereof. 


\section{DISCLAIMER}

Portions of this document may be illegible in electronic image products. Images are produced from the best available original document. 


\begin{abstract}
Thermal Hydraulic Characteristics Study of Prototype NET and CEA Cable-in-Conduit Conductors (CICCs)
\end{abstract}

\author{
Ryuji Maekawa \\ Under the supervision of Professor Steven W. Van Sciver \\ at the University of Wisconsin-Madison
}

The thermal hydraulic characteristics of low temperature helium in a Cable-in-Conduit Conductor (CICC) significantly affects the overall design and performance of the associated - large scale superconducting magnet system. It is essential to understand the transient and steady state behavior of the helium in the conductor. Throughout the development of CICCs, the reduction of flow impedance has been one of the key factors to improving the overall pressure drop. The newly developed CICC for the ITER project has a hybrid cooling scheme: a central channel that is surrounded by bundles, for which the thermal hydraulic characteristics are not well understood.

This thesis describes an experimental and analytical investigation of thermal hydraulic characteristics of low temperature helium in conventional and hybrid CICCs. Pressure drop measurements for both NET and CEA conductors have been conducted, using low temperature helium and liquid nitrogen to obtain a range of Reynolds numbers. The results are correlated with classical friction factor and Reynolds number analysis. The flow 
-impedance-reduction-of-the-EEA-conductor-is-deseribed-by-means-of-a-developed-flow-model.

Thermally induced flow in the CEA conductor has been studied with an inductive heating method. The induced velocity in the central channel is measured by a Pitot tube with steady state Reynolds number up to $\sim 7000$. The transient pressure wave propagation has been recorded with pressure transducers placed equally along the conductor. The supercritical helium temperature in the central channel has been measured with the thermometer probe. However, the reduction of the central channel area significantly affects the overall thermal hydraulic characteristics of the conductor. The results suggest the importance of the central channel.

$\mathrm{A}$ transient heat transfer experiment studied the transverse heat transfer mechanism in the CEA conductor. The temperatures in the central channel and bundle region were measured with different sized heat pulses. The results are discussed in terms of a heat transfer model which is based upon the communication of the helium in the central channel and bundle.

Approved:

Date / /

Steven W. Van Sciver, Professor

Nuclear Engineering and Engineering Physics 


\section{Acknowledgments}

First of all, I would like to thank my advisor, Professor Steven W. Van Sciver. I am so fortunate to have the benefit of your precious guidance, expertise, encouragement and support. I learned so much from you. Both of us have a back problem and I really hope your back is not getting worse. Hopefully, I can provide you some new treatments after I go back to Japan.

I also would like to thank my committee members: Professor, Robert Witt, John Pfotenhauer, Michael Corradini and David Larbalestier, for taking time to review my thesis and come to my defense.

Since I joined our group as a last student at University of Wisconsin-Madison, I have to be a rookie for three long years. Fortunately, our group has such great graduate students, I learned a lot from them: Mark Daugherty, Dave Pearson, Xiaodong Huang and Yuenian Huang. I have grown so much both working with them and knowing them. Thank you very much. I also thank to Dr. Dwight Kingsbury to check my prelim.

My last three years of study was taken place at National High Magnetic Field Lab. I would like to thank Dr. Michael R. Smith for his support. I would like to express my appreciation to John Panek for helping me to cool down the LHFF. (Thanks for carrying a heavy liquid nitrogen dewar.) Thanks to Philip Shoaff for giving me an opportunity to join the Sailing club. Also thanks to Bertrand Baudouy who was my mid-night discussion buddy while I was writing my thesis. Thanks to Scott Welton, my occasional tennis partner, who was always blaming me about new tennis balls which I bought. I think the real problem is something else. Also thanks to Keith Bartholomew who helped me to prepare for my experiment. Thank you to drive a folk lift for me.

I also express my gratitude to Alfred Kleinhammes who has been a great hitting partner/ ; beer drinker/psychiatrist, etc. Thanks for everything. I would like to thank my team mates: 
Leo Dean and Gary Mower, who supported me on the tennis court. However, I am not sure what we were trying to prove to play tennis at eleven o'clock in the strong Florida sunshine.

Thank to Keiko Onoda who supported me in my early graduate study which turned out to be the hardest time except writing my thesis. Thank you so much.

Many thanks to Professor Yoich Matsubara at Nihon University, who encouraged me to work for my Ph.D as well as finding me an opportunity to work at National Institute for Fusion Science. I am truly indebted to you.

Finally, I would like to thank my parents, Tokiko and Norio Maekawa, for letting me study in the United States. Thanks so much for believing in me. I also thanks to my brother, Katsunori, and his wife, Reiko, for their warm supports. 
Dedicated to my Mother and Father

Tokiko and Norio Maekawa 
Table of Contents

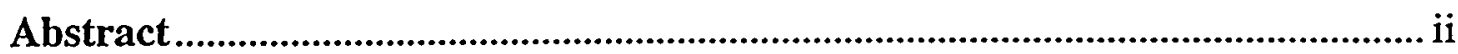

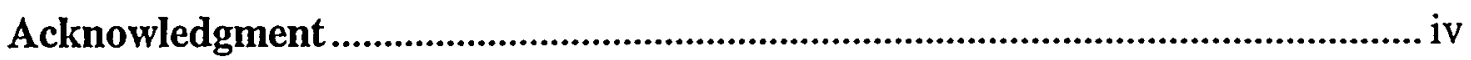

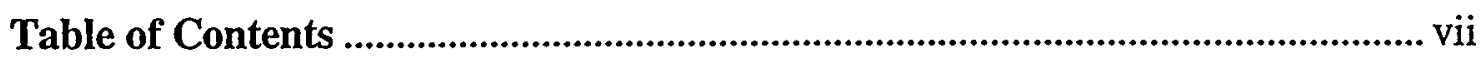

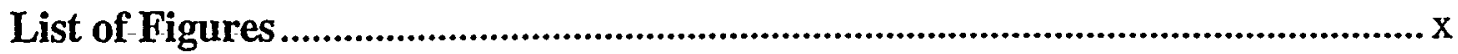

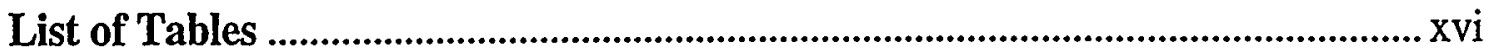

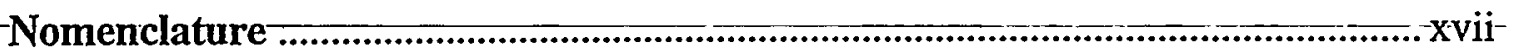

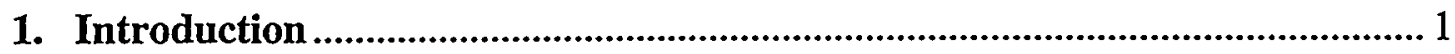

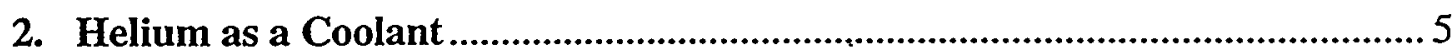

2.1 Fundamentals of Helium ..................................................... 5

2.2 Helium II ................................................................................. 7

2.2.1 The Two Fluid Model ....................................................... 7

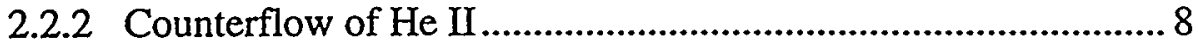

2.2.3 Steady State Heat Transfer ............................................ 10

2.2.4 Transient Heat Transfer .................................................... 12

2.2.5 Kapitza Conductance ....................................................... 13

2.3 Supercritical Helium as a Coolant ...................................................... 16

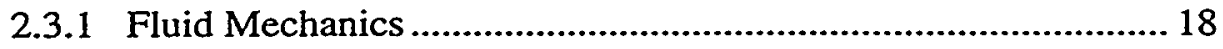

2.3.2 Forced Convection Heat Transfer ..................................... 19

2.3.3 Transient Heat Transfer ................................................. 21

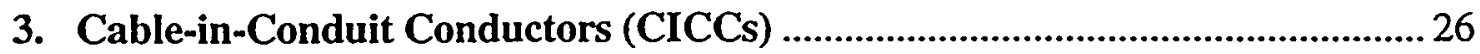

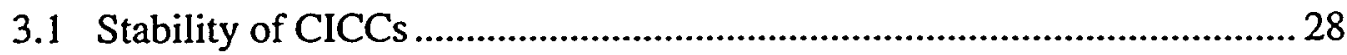

3.1.1 Induced Flow of Supercritical Helium ...................................29

3.1.2 Multivalued Stability ........................................................ 33 


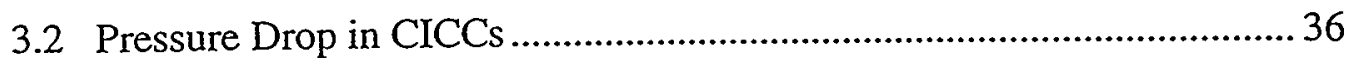

3.3 New Type Cable-in-Conduit Conductor …………................................... 44

4. Description of Experiments .............................................................................. 48

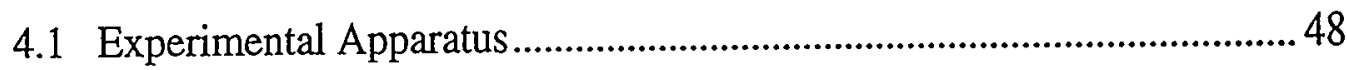

4.1.1 Liquid Helium Flow Facility .................................................... 48

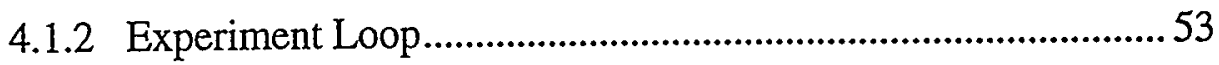

4.1.3 Transient Heat Transfer Experiment............................................55

4.1.4 Counterflow Heat Transport Experiment.....................................58

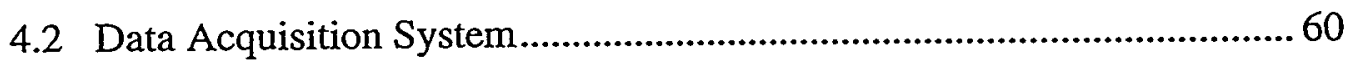

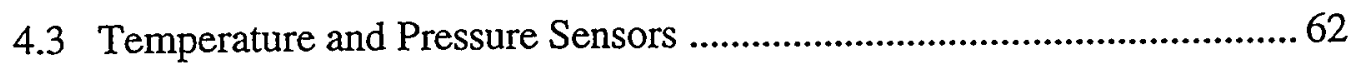

4.3.1 Germanium Resistance Thermometer.......................................... 62

4.3.2 Allen Bradley Thermometer …………………........................... 63

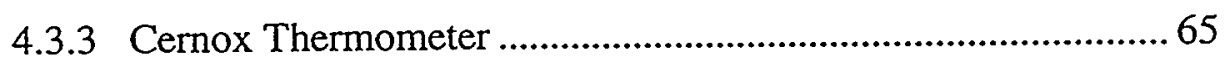

4.3.4 Calibration of Temperature Sensors ..........................................66

4.3.5 Variable Reluctance Pressure Transducer.................................... 68

4.3.6 Cryogenic Pressure Transducer ................................................... 70

4.3.7 Siemens KPY Pressure Transducer.............................................. 70

4.4 Experimental Procedure .......................................................................... 71

4.4.1 Counterflow Heat Transport Experiment .................................... 72

4.4.2 Pressure Drop Measurement ....................................................... 73

4.4.3 Inductive Heater Transient Pressure Rise Experiment .............. 73

4.4.4 Transient Heat Transfer Experiment ............................................ 73

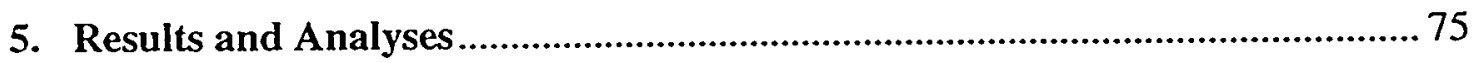

5.1 Characterization of the NET and CEA Cable-in-Conduit Conductors .... 75

5.1.1 Image and Geometrical Component Analyses ............................. 76

5.1.2 Counterflow Heat Transport Analysis ......................................... 79 


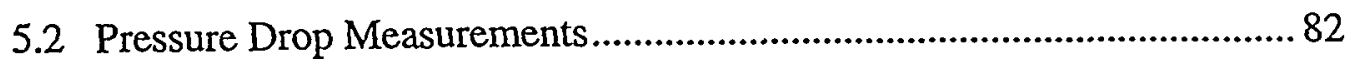

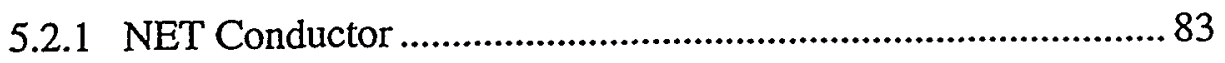

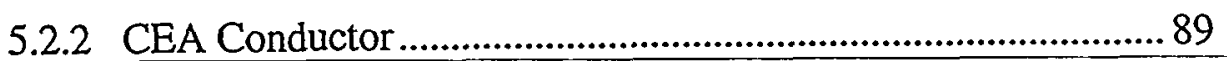

5.2.3 Modeling Flow in the CEA Conductor .................................. 99

5.2.4 Comparison of the NET and the CEA conductor .................... 107

5.3 Inductive Heater Experiment ......................................................... 110

5.3.1 Calibration of the Inductive Heater ...................................... 110

5.3.2 Thermally Induced Flow Experiment ..................................... 114

5.3.2.1 Stagnant Supercritical Helium and He II ................... 115

5.3.2.2 Forced Flow Supercritical Helium ........................... 124

5.3.3 Induced Flow with the Thermometer Probe in the Central Channel......................................................... 133

5.4 Transient Heat Transfer Experiment ................................................... 142

5.4.1 Saturated He II .............................................................. 142

5.4.2 Supercritical Helium .......................................................... 144

5.4.3 Transverse Heat Transfer in the CEA conductor ...................... 147

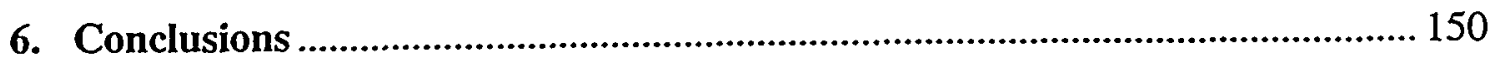

6.1 Hydraulic Characteristics of CICCs ..................................................... 150

6.2 Thermally Induced Flow in the CEA Conductor ................................... 151

6.3 Transient Heat Transfer ...................................................................... 153

6.4 Suggested Future Work..................................................................... 153

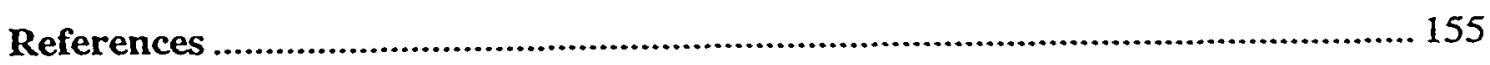




\section{List of Figures}

1.1 Upper critical field, temperature and current density for commercial superconducting materials: $\mathrm{NbTi}$ and $\mathrm{Nb}_{3} \mathrm{Sn}$....................................... 2

1.2 Cross section of a typical monolith conductor ......................................... 3

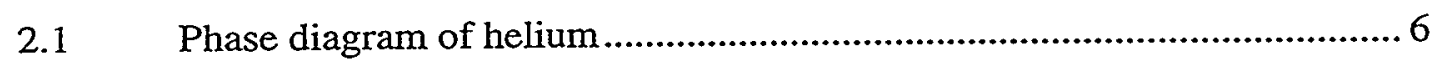

2.2 Steady state heat transport in He II ....................................................... 10

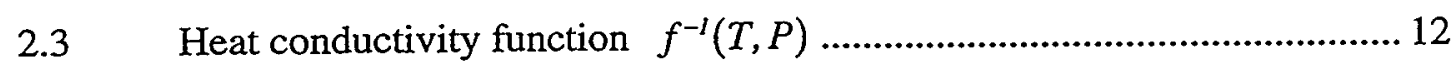

2.4 Comparison of Kapitza conductance with the theory and experimental results ...................................................................... 15

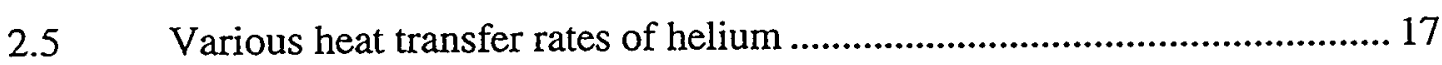

2.6 Pressure drop and Temperature gradient of supercritical helium

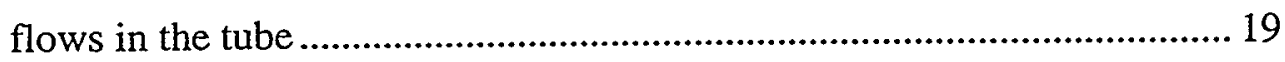

2.7 Comparison of experimental results and an empirical correlation ............20

2.8 Comparison of transient heat transfer coefficient ...................................22

2.9 Comparison of experimental results with Equation (2.31) .......................25

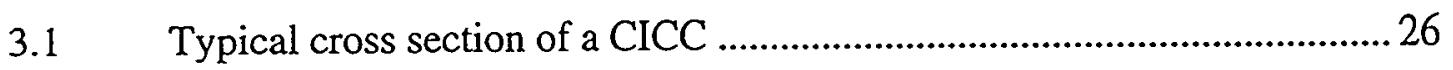

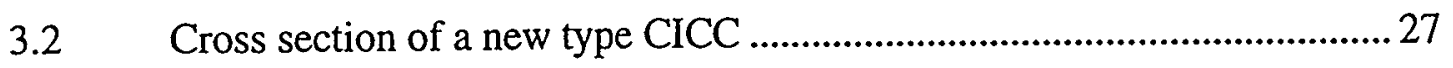

3.3 Heat transfer coefficient from Equation (3.9) and

experimental results ........................................................................ 31

3.4 Numerical calculation of the induced mass flow rate

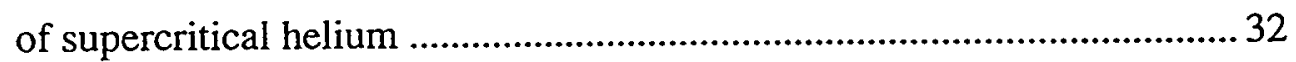

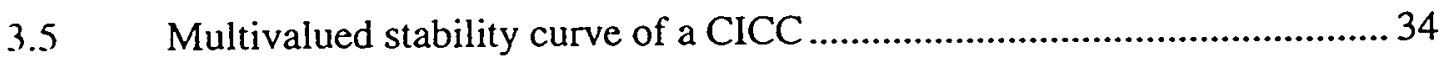

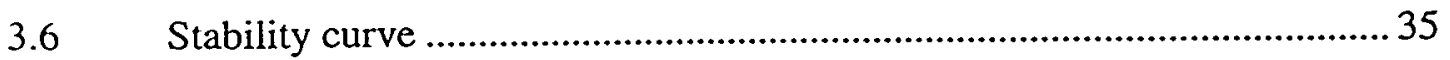

Imposed heat transfer coefficient .......................................................... 35 
3.8 The origin of multivalued stability curve

3.9 Friction factor vs. Reynolds number correlation from Lue et al. ................ 38

3.10 Friction factor vs. Reynolds number for DPC-TJ ........................................ 39

3.11 Katheder's correlation compared with experimental results........................40

3.12 f vs. Re for the CICCs with different twist pitch .......................................... 41

3.13 Friction factor vs. Reynolds number for CICCs with apparent

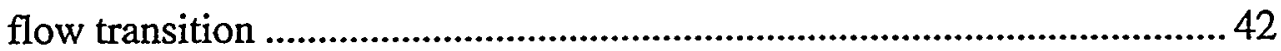

3.13 Friction factor vs. Reynolds number for Toki-PF....................................... 43

3.14 Friction factor vs. excitation current of the coil .......................................... 43

3.15 Friction factor vs. excitation number of the coil ...........................................4 43

3.16 Train effects ..........................................................................................4 46

3.17 Mass flow rate vs. time after the quench ………......................................... 47

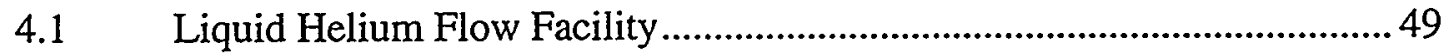

4.2 A centrifugal liquid helium pump …….....................................................5

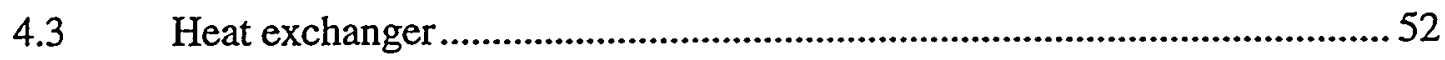

4.4 Schematic view of a test loop ...............................................................5

4.5 Transient heat transfer experimental apparatus ...........................................56

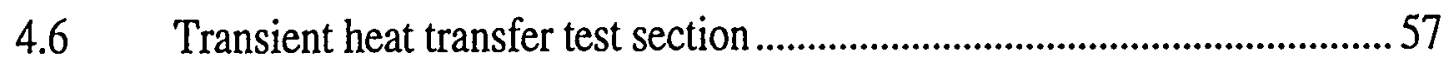

4.7 Test section of a counterflow heat transport experiment ...........................59

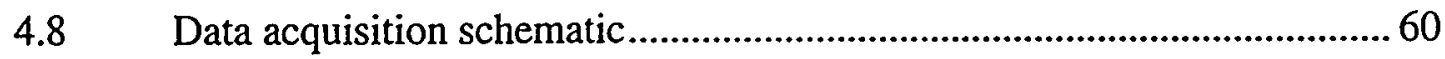

4.9 Typical wiring scheme for analog data input ...............................................61

4.10 Calibration curve and data points for a germanium thermometer ...............63

4.11 Typical calibration curve for an Allen Bradley thermometer ......................64 64

4.12 Dimension of cernox thin film thermometer..............................................65

4.13 Calibration curve for the Cernox thermometer ..............................................6

4.14 Diagram of a pressure sensor calibration ....................................................69 
5.1 Binary image of NET conductor .............................................................. 77

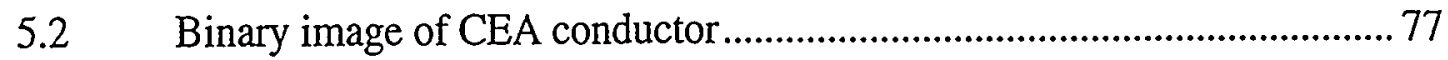

5.3a The heat flux vs. temperatuire difference fot $\mathrm{Tb}=1.8 \mathrm{~K} \ldots \ldots \ldots \ldots \ldots \ldots \ldots \ldots \ldots . . . . . . . . . . . .80$

5.3b The heat flux vs. temperatuire difference fot $\mathrm{Tb}=1.9 \mathrm{~K}$............................. 80

5.3c The heat flux vs. temperatuire difference fot $\mathrm{Tb}=2.05 \mathrm{~K}$.......................... 81

5.4 The effective heat transfer area as a function of $\mathrm{He}$ II temperature ............ 81

5.5 He II temperature vs. mass flow rate .......................................................... 84

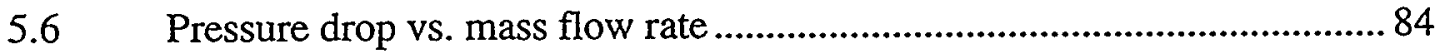

5.7 Pressure drop vs. average He II velocity ..................................................... 85

5.8 Friction factor vs. Reynolds number for the NET conductor ......................8 87

5.9 Comparison of friction factor variation with different flow area analyses .................................................................................... 88

5.10 Supercritical helium temperature vs. mass flow rate …................................ 89

5.11 Static pressure in the conductor vs. mass flow rate ................................... 90

5.12 Pressure drop vs. mass flow rate for different runs...................................... 91

5.13 Pressure drop vs. supercritical helium velocity ........................................92

5.14 Fraction of mass flow rate in the central channel vs.

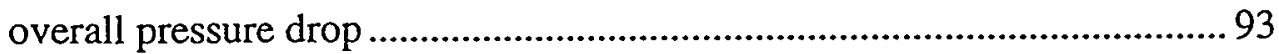

5.15 Comparison of supercritical helium velocity in the central channel and the rest of the conductor .......................................................................... 94

5.16 Hydraulic diameter of the central channel and the conductor ...................95

5.17 Six point star duct with a hydraulic diameter …...........................................96

5.18 Friction factor vs. Reynolds number for the CEA conductor .................... 98

5.19 Comparison of different flow area analysis on friction factor variation .... 99

5.20 Cross section for the flow modeling in the CEA conductor ....................... 101

5.21 Comparison of two models (pressure drop vs. mass flow rate) ................... 104 
5.22 Pressure drop vs. mass flow rate (model and experimental results) .......... 104

5.23 Comparison of a flow model with experimental results in

the frction factor vs. Reynolds number ................................................... 106

5.24 Comparison of flow velocity in the central channel ................................ 107

5.25 Friction factor vs. Reynolds number for NET and CEA conductor........... 109

5.26 Equivalent electrical circuit for the inductive heating ............................. 110

$5.27 \quad$ Typical calibration curve with stored energy $~ 560 \mathrm{~J}$............................. 111

Inductive heater calibration results ................................................. 112

5.29 Estimated $\mathrm{Q}(\mathrm{t})$ and $\mathrm{H}(\mathrm{t})$ from measured voltage across the resistor .......... 114

5.30a Mass flow rate and v_central vs. time (8 J) ......................................... 115

5.30b Mass flow rate and v_central vs. time (19.5 J) ................................. 116

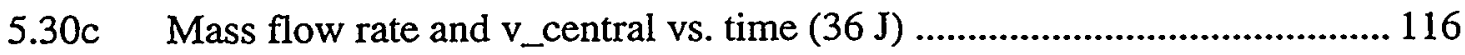

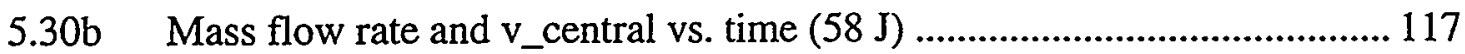

5.31a Static pressure vs. time for shot \#16 .................................................... 117

5.31b Static pressure vs. time for shot \#17 .............................................. 118

5.32a Mass flow rate vs. time for shot \#14 ................................................... 119

5.32b Mass flow rate vs. time for shot \#15 ...................................................... 119

5.32c Mass flow rate vs. time for shot \#16 …............................................... 120

5.33 Thermal hydraulic behavior of supercritical helium in the conductor ....... 120

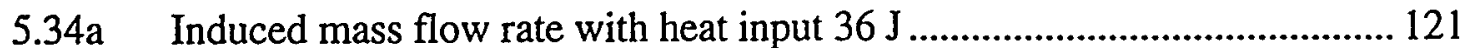

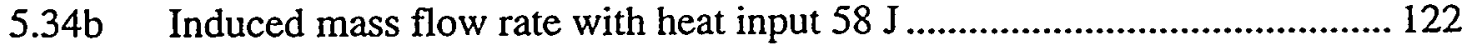

5.35 Induced flow velocity in He II with different energy input ....................... 122

5.36 Comparison of thermally induced flow velocity with

Dresner's solution

5.37a Induced total mass flow rate and velocity in the central channel $(8 \mathrm{~J}) \ldots 125$

$5.37 \mathrm{~b}$ Induced total mass flow rate and velocity in the central channel 
$(19.5 \mathrm{~J})$

5.37c Induced total mass flow rate and velocity in the central channel

$(36.5 \mathrm{~J})$ 126

5.38a Induced total mass flow rate and velocity in the central channel (8 J) ...... 126

5.38b Induced total mass flow rate and velocity in the central channel (19.5 J) 127

$5.38 \mathrm{c}$ Induced total mass flow rate and velocity in the central channel ( $36.5 \mathrm{~J}) .127$

5.39a Induced flow velocity in the central channel $(\operatorname{Re} ~ 5100)$............................. 128

$5.39 \mathrm{~b}$ Induced flow velocity in the central channel $(\operatorname{Re} ~ 7050)$............................ 128

5.40 The fractional increase of induced flow velocity vs. energy input ............. 129

5.41a Comparison of mass flow rate in each region (8 J) .................................... 130

5.41b Comparison of mass flow rate in each region (19.5 J) ................................. 130

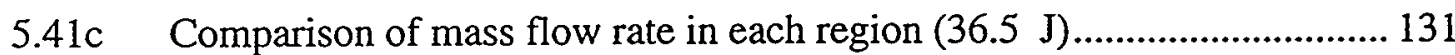

5.42 Comparison of velocity in each channel (36.5 J) ........................................ 131

5.43a Static pressure change along the conductor after the heat pulse (19.5 J)... 132

$5.43 \mathrm{~b}$ Static pressure change along the conductor after the heat pulse ( $36.5 \mathrm{~J}) \ldots 132$

5.44 Induced mass flow with different heat input $(\operatorname{Re} \sim 6000)$........................... 134

5.45 Induced mass flow with different heat input $(\operatorname{Re} \sim 17000)$

5.46 The relationship between induced flow and mass flow rate ........................ 136

5.47 The relation of induced velocity to steady state velocity

as a function of heat input and imposed mass flow rate

5.48 Fraction of flow rate reduction with different

mass flow rate and heat input

5.49a Temperature profile in the central channel (36.5 J) ................................... 138

$5.49 \mathrm{~b}$ Temperature profile in the central channel $(58.3 \mathrm{~J})$.................................... 138

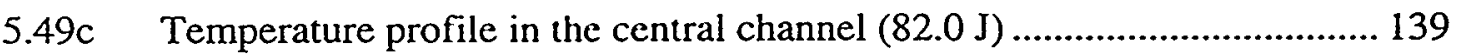

5.50 Induced flow velocity calculated by time of flight ..................................... 140 
5.51 Velocity variation along the conductor calculated by time of flight........... 141

5.52 Temperature profile after the heat pulse (He II) .................................. 143

5.53 Heat transfer coefficient (He II) ......................................................... 144

5.54 Strands temperature profile after heat pulse ...................................... 145

5.55 Supercritical helium temperature in the central channel......................... 146

5.56 Heat transfer coefficient (supercritical helium) ............................... 147

Comparison of heat transfer coefficient .............................................. 149 


\section{List of Tables}

$5.1 \quad$ The image analysis results with some other parameters ......................... 78

$5.2 \quad$ Nominal geometric component calculation results ............................... 78

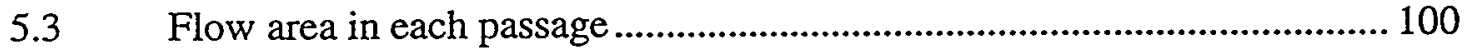




\section{Nomenclature}

\begin{tabular}{ll}
$A$ & Relative roughness constant \\
$A_{\text {flow }}$ & Helium flow area \\
$A_{G-M}$ & Gorter-Mellink mutual friction coefficient \\
$C$ & Numerical constant \\
$C_{p}$ & Specific heat capacity at constant pressure \\
$D$ & Diameter \\
$D_{l}$ & Hydraulic diameter \\
$f$ & Friction factor \\
$H$ & Enthalpy \\
$h$ & Heat transfer coefficient \\
$\bar{h}$ & Average heat transfer coefficient \\
$h$ & Specific enthalpy \\
$h_{K}$ & Kapitza conductance \\
$j$ & Momentum density \\
$k$ & Thermal conductivity \\
$\dot{m}$ & Mass flow rate \\
$N u$ & Nusselt number \\
$P_{c}$ & Critical pressure \\
$P r$ & Prandtl number \\
$\Delta P$ & Pressure drop \\
$P_{c u l l c d}$ & Cooled perimeter \\
$Q$ & Total heat \\
$q$ & Heat flux \\
\hline$P$ & \\
\hline &
\end{tabular}


$q^{*} \quad$ Maximum heat flux

$q_{f} \quad$ Heat flux to the fluid

$q_{s} \quad$ Heat flux to the substrate

$R \quad$ Electrical resistance

$r \quad$ Radius

Re Reynolds number

$s \quad$ Entropy

$T_{b} \quad$ Bath temperature

$T_{c} \quad$ Critical temperature

$\Delta T_{s} \quad$ Temperature difference at the interface

$T_{w} \quad$ Wall temperature

$T_{\lambda} \quad$ Lambda temperature

u Velocity

V Voltage

$v_{n} \quad$ Normal component velocity

$v_{s} \quad$ Superfluid component velocity

$v_{\text {void }} \quad$ Void fraction

$\alpha \quad$ Thermal Diffusivity

$\beta \quad$ Bulk expansivity

$\eta \quad$ Viscosity

$\rho \quad$ Density

Bold type indicates vector quantities. 


\section{Introduction}

This thesis presents an experimental and analytical study of the thermal hydraulic characteristics of two prototype Cable-in-Conduit Conductors (CICCs) cooled by the forced flow low temperature helium. The investigation includes: flow area characterization of conductors; pressure drop measurements and flow modeling; thermally-induced flow and transient pressure rise experiment; and transient heat transfer experiment. The goal is to improve understanding of the thermal-hydraulic behavior of CICCs.

CICCs are widely used for the large scale superconducting magnet systems because of their mechanical strength and high transport current (above $10 \mathrm{kA}$ ). The primary application of these conductors includes: poloidal, toroidal field coils and central solenoid for Tokamak fusion machines such as ITER (International Thermonuclear Experimental Reactor), PF coils for a heliotron/torsatron type experimental fusion reactor, LHD (Large Helical Device) at NIFS (National Institute for Fusion Science), SMES (Superconducting Magnetic Energy Storage) and high field magnet facilities; e.g. 45-T Hybrid at NHMFL(National High Magnetic Field Laboratory). Understanding the stability and quench characteristics of CICCs is an important issue for reliable superconducting magnet design and operation.

Superconductors have the ability to transport very high current without dissipating any heat because of their near zero electrical resistivity below the critical temperature, $\mathrm{T}_{\mathrm{c}}$. The superconducting state depends on the temperature, magnetic field and current density as shown in Figure 1.1. Under the surface area represents superconducting state and outside is normal state. Note that the helium is the only practical fluid to provide a refrigeration for commercially available superconductors; $\mathrm{NbTi}$ and $\mathrm{Nb}_{3} \mathrm{Sn}$. Understanding the thermal fluid interaction between a superconducting cable and the helium coolant is crucial to the proper design of a superconducting magnet. 


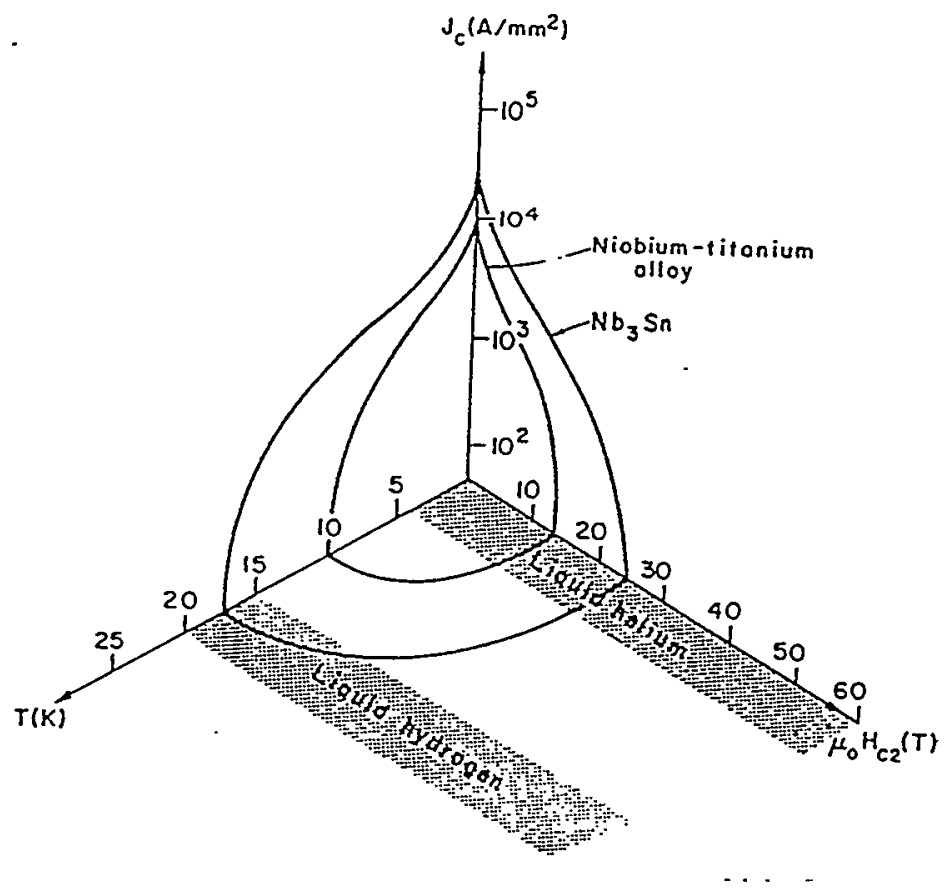

Figure 1.1. Upper critical field, temperature and current density for commercial superconducting materials: $\mathrm{NbTi}_{\text {and }} \mathrm{Nb}_{3} \mathrm{Sn} .{ }^{1}$

Superconductors have high electrical resistivities in the normal state. This requires that either copper or high purity aluminum be used as a stabilizer in a monolithic type conductor, with the superconductor distributed in filaments as shown in Figure 1.2. The CICC is comprised of many monolithic conductor strands encased in the tight fitting conduit that is made of high strength alloy, such as stainless steel. The forced flow supercritical helium circulates in the conduit to provide the refrigeration to the strands. 


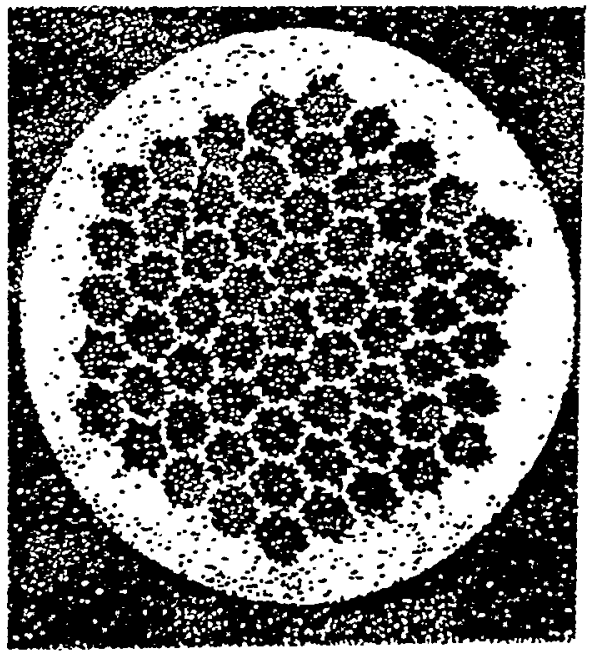

Figure 1.2. Cross section of a typical monolith conductor. ${ }^{2}$

Superconductor current density is a very important factor in the design of superconducting magnets; since it affects the cost and the size of the magnet. High current density is essential for the large scale magnets. At the same time, the proper protection of the magnet is essential for magnet quench during which the whole conductor may become normal.

Since the specific heat of the metal is very small at helium temperature $\sim 4.2 \mathrm{~K}$, strand movement due to the Lorentz force may raise the superconductor temperature close to the current sharing temperature, at which a portion of the current starts to flow in the stabilizer. Joule heat is generated at this point. If the available helium enthalpy is not sufficient, the superconductor temperature will increase and a part of superconductor will become normal, this is called a normal zone. The minimum propagating zone (MPZ), i.e. the smallest stable expanding normal zone developed within the conductor, is used to quantify the stability of the conductor against such a disturbance. If the point disturbance creates a normal zone 
which is larger than the MPZ, the normal zone will propagate along the conductor and quench the magnet. This is an irreversible process.

In the case of sufficient cooling of the strands, the normal zone will collapse and the superconductor will recover its superconducting state. This process primary depends on the transient heat transfer to the helium and the associated pressure rise in the CICCs. When the transient thermal disturbance is locally generated, the helium will absorb it and expand, which in turn will induce helium flow within the CICC. This thermally induced flow of the helium enhances the heat transfer between the strands and the helium, which can aid the stability of the conductor.

However, the helium motion within the conductor is not easy to define, since the induced flow and compression of helium vary along the CICC cooling path. In addition, thermallyinduced flow of helium and the normal zone growth are coupled processes with complicated spatial and temporal dependence. The process requires fairly complex numerical analysis to describe the transient thermal hydraulic behavior of supercritical helium in the CICC. In addition, solution of the associated hydrodynamic equations is a challenge since the characteristics of supercritical helium are strongly dependent on both temperature and pressure.

Throughout the development of CICCs, it has been essential to benchmark codes with experimental results. Thus, the present work is focused on providing the experimental data to further understanding of the thermal-hydraulic characteristics of CICCs. 


\section{Helium as a Coolant}

Liquid helium is widely used as a coolant to operate superconducting magnets. Frequently, a superconducting magnet is immersed in a liquid helium bath under atmospheric pressure. This approach is called the pool boiling method. Another type of a cooling method, forced flow cooling, is mainly used in modern large scale superconducting magnets. In this case, the forced flow supercritical helium is inside a thick-wall stainless steel tube which contains a number of superconducting strands. This method requires much more complex refrigeration systems than does the pool boiling because of circulation of low temperature helium within a very long cooling path of a superconducting magnet. Therefore, it is crucial to understand the fluid dynamics as well as the heat transfer mechanisms of low temperature helium to design cryogenic systems for the forced flow cooling type magnets. The present chapter is a summary of the fundamental characteristics: fluid mechanics; steady-state heat transfer; forcedconvection heat transfer and transient heat transfer mechanism of low temperature helium.

\subsection{Fundamentals of Helium}

Many characteristics of helium $\left({ }^{4} \mathrm{He}\right)$ can be described by near ideal gas or fluid models; a fact resulting from the weak inter-atomic potential and a spherical symmetric molecular configuration. In fact, helium has the lowest critical point, $\mathrm{Tc}=5.2 \mathrm{~K}$ at $\mathrm{Pc}=0.226 \mathrm{MPa}$, of

all other cryogenic fluids (except the rare isotope ${ }^{3} \mathrm{He}$ which has $\mathrm{Tc}=3.324 \mathrm{~K}$ at $\mathrm{Pc}=0.116$ MPa). Figure 2.1 shows the phase diagram of helium. As shown in the figure, liquid helium can cxist in either of two states: He I or He II. Normal liquid helium, or He I, is not very different from the gaseous state because of a low density and low viscosity. The density of He I is approximately 7.4 times larger than its coexisting vapor at normal boiling point $(\mathrm{T}=4.2$ 
$\mathrm{K}, \mathrm{P} \sim 100 \mathrm{kPa}$ ). In addition, the viscosity of liquid helium is comparable to that of air at room temperature.

On the other hand, superfluid helium or He II, shows totally different characteristics to those of all other ordinary fluids. The intersection line between $\mathrm{He} \mathrm{I}$ and $\mathrm{He}$ II is called lambda $(\lambda)$ line since the shape of the specific heat curve at transition temperature region looks like a Greek letter $\lambda$. The phase transition at the $\lambda$-point is second order and there is no latent heat. Therefore, He I and He II can not co-exist in equilibrium. The important thing to note is that the lowest energy state is liquid and helium can only become solid if applied pressure exceeds 2.5 MPa. This implies that helium also possesses quantum characteristics; such as a large zero point energy. ${ }^{3}$

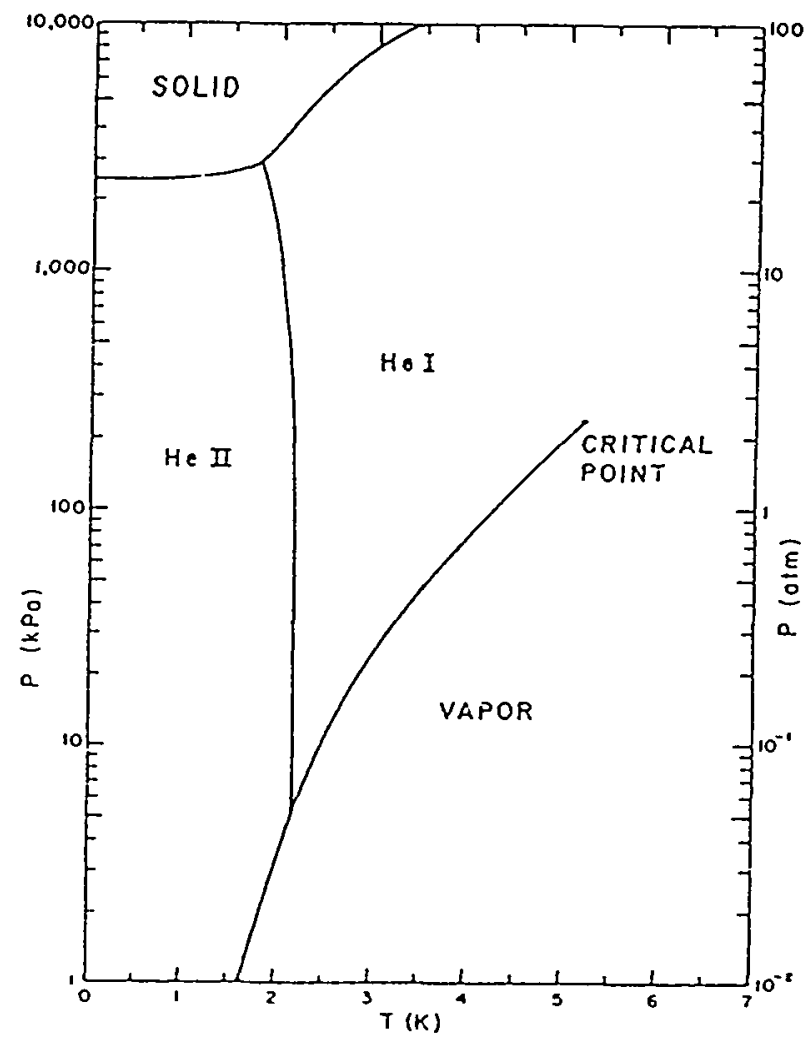

Figure 2.1. Phase diagram of helium. ${ }^{3}$ 


\subsection{Helium II}

Unlike other ordinary fluids, He II possess quite unique characteristics. Some of those distinct characteristics are as follows. First, He II can flow through narrow capillary tubes with very small pressure differences which implies that $\mathrm{He} I$ has extremely small viscosity. 4,5 Second, He II has extremely high effective thermal conductivity, in other words, He II can transport a large heat flux with a very small temperature gradient. ${ }^{6}$ Third, He II can transmit second sound, which is the propagation of thermal waves caused by the fluctuation of the local entropy. ${ }^{7}$

\subsubsection{The Two Fluid Model}

The two fluid model ${ }^{8}$ provides good insight about transport properties of $\mathrm{He}$ II, which suggests that the helium consists of intimate mixture of two fluids: normal fluid and superfluid. The normal component has density $\rho_{n}$, viscosity $\eta_{n}$ and specific entropy $s_{n}$ as similar to that of ordinary fluids. The superfluid component, however, has density $\rho_{s}$ but has neither a viscosity nor an entropy. The density of $\mathrm{He}$ II is expressed as the sum of two components,

$$
\rho=\rho_{n}+\rho_{s}
$$

Concentrations of both fluid components are strongly temperature dependent. Experiments have shown that the ratio of normal fluid and superfluid densities to the total may be expressed as,

$$
\frac{\rho_{n}}{\rho}=\left(\frac{T}{T_{\lambda}}\right)^{5.6}
$$




$$
\frac{\rho_{n}}{\rho}=\left(\frac{T}{T_{\lambda}}\right)^{5.6}
$$

and

$$
\frac{\rho_{s}}{\rho}=1-\left(\frac{T}{T_{\lambda}}\right)^{5.6}
$$

At temperature is lower than $1 \mathrm{~K}$, the concentration of normal fluid components is negligibly small.

\subsubsection{Counterflow of $\mathrm{He}$ II}

The two-fluid model explains that the extremely high thermal conductivity of $\mathrm{He}$ II in terms of counterflow or internal convection of normal fluid and superfluid components. If a heat source is present within stagnant He II, the normal fluid flows away from the heat source with a velocity $v_{n}$. Therefore, the velocity $v_{n}$ can be expressed as a function of a heat flux density $q$,

$$
v_{n}=\frac{q}{\rho s T}
$$

Since there is no net mass flow, the total or net momentum density is zero.

$$
j=\rho v=\rho_{\mathrm{n}} v_{n}+\rho_{s} v_{s}=0
$$

Consequently, superfluid flows towards the heat source with a velocity,

$$
v_{s}=-\frac{\rho_{n}}{\rho_{s}} v_{n}
$$


There are two different frictional processes of the heat transport of He II. The first is the viscous interaction of normal fluid component with the channel walls, which is the only dissipation term at very small heat flux $\left(\sim 1 \mathrm{~W} / \mathrm{m}^{2}\right)$. In this case, the heat flux in a tube of radius $r$ is expressed as a linear function of the temperature gradients,

$$
q=-\frac{r^{2} \rho^{2} s^{2} T}{8 \eta_{n}} \nabla T
$$

Another process is the mutual friction of normal fluid and superfluid components. Once a heat flux $q$ increases above a critical value $q_{c}$, the superfluid component develops a tangled mass of quantized vortex lines which interact with normal fluid. The turbulence in He II is developed above the critical relative velocity of two components $\left|v_{s}-v_{n}\right|_{c}$. This process gives the Gorter-Mellink mutual friction, ${ }^{9}$

$$
f=A \rho_{n} \rho_{s}\left|v_{s}-v_{n}\right|^{3}
$$

where $A$ is an empirical numerical coefficient.

An expression for the temperature gradient under steady state conditions can be expressed as the velocities of each component;

$$
\nabla T=-\frac{8 \eta_{n}\left|v_{n}\right|}{r^{2} \rho s}-\frac{A \rho_{n}}{s}\left|v_{s}-v_{n}\right|^{3}
$$

Using Equation (2.3), the general expression of above equation in terms of a heat flux $q$ is given by,

$$
-\nabla T=\frac{8 \eta_{n} q}{r^{2} \rho^{2} s^{2} T}+\frac{A \rho_{n}}{\rho_{s}^{3} s^{4} T^{3}} q^{3}
$$


This is the heat transport equation of He II under steady-state condition. Note that for large heat flux $q$, the second term of the right hand side of Equation (2.9) dominates.

\subsubsection{Steady State Heat Transfer}

Although He II has extremely high effective thermal conductivity, a maximum heat flux exists since He II must be maintained below the $\lambda$-temperature. To understand steady-state heat transport in He II, let us assume a channel of length $L$ containing $\mathrm{He}$ II with one end connected to He II bath. A constant heat flux $q$ is applied to the other end of a channel as shown in Figure 2.2.

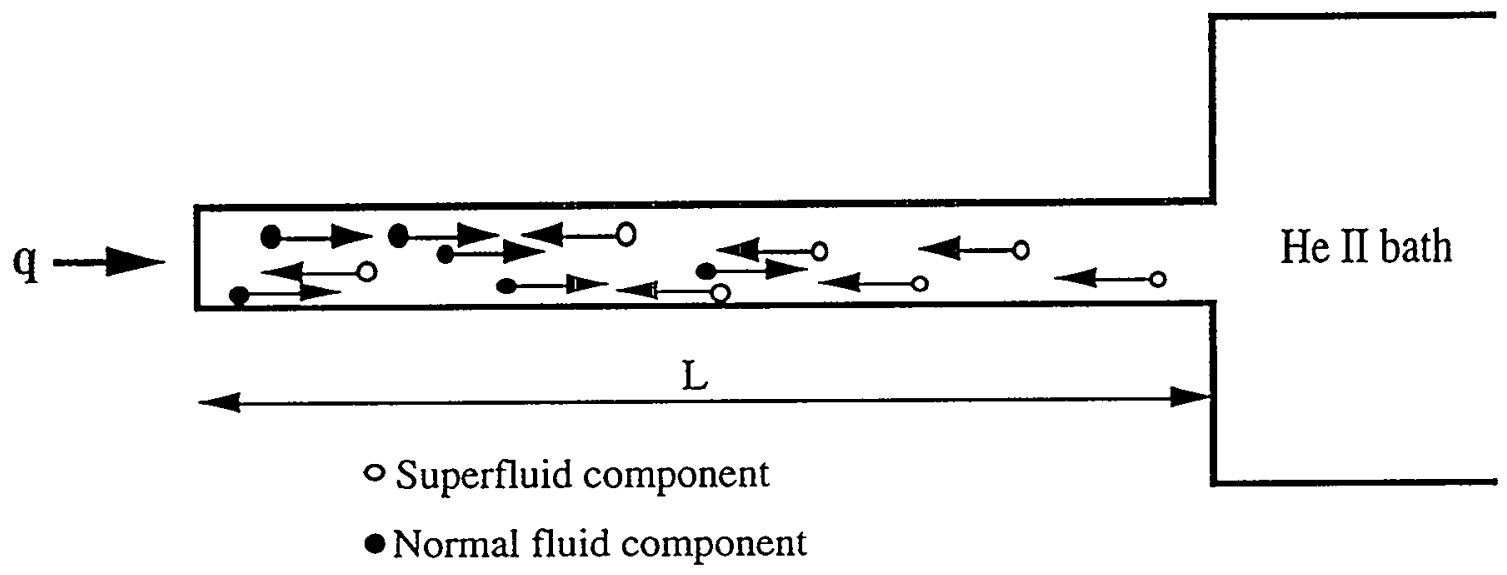

Figure 2.2. Steady state heat transport in He II.

As explained at the previous section, a temperature gradient is established along the channel by the normal fluid viscous interaction with a channel wall and the mutual friction between normal fluid and superfluid component. However, in case of this interest to most application, the mutual friction term dominates since the heat flux is large enough to generate 
turbulence in He II. Therefore, the one-dimensional turbulent heat transport equation (2.9) becomes;

$$
-\frac{d T}{d x}=f(T) q^{3}
$$

where the heat conductivity function $f^{-1}(T, P)$ is expressed as

$$
f^{-1}(T, P)=\frac{\rho^{2} s \lambda^{4} T \lambda^{3}}{A_{\lambda}}\left\{t^{5.7}\left(1-t^{5.7}\right)\right\}^{3}
$$

with $t=T / T_{\lambda}$. Figure 2.3 shows $f^{-1}(T, P)$ curves at different pressures. It is important to realize the analytic expression for the heat conductivity function is only accurate to $\pm 10 \%$ at saturated vapor pressure.

It is possible to estimate the maximum heat flux $q^{*}$ within a channel length $L$, using equations (2.10) and (2.11). Integrating Equation (2.10) over the channel length, one obtains,

$$
q^{*}=\left(\frac{1}{L} \int_{T_{b}}^{T_{2}} \frac{d T}{f(T)}\right)^{1 / 3}
$$

where $T_{b}$ is a He II bath temperature. 


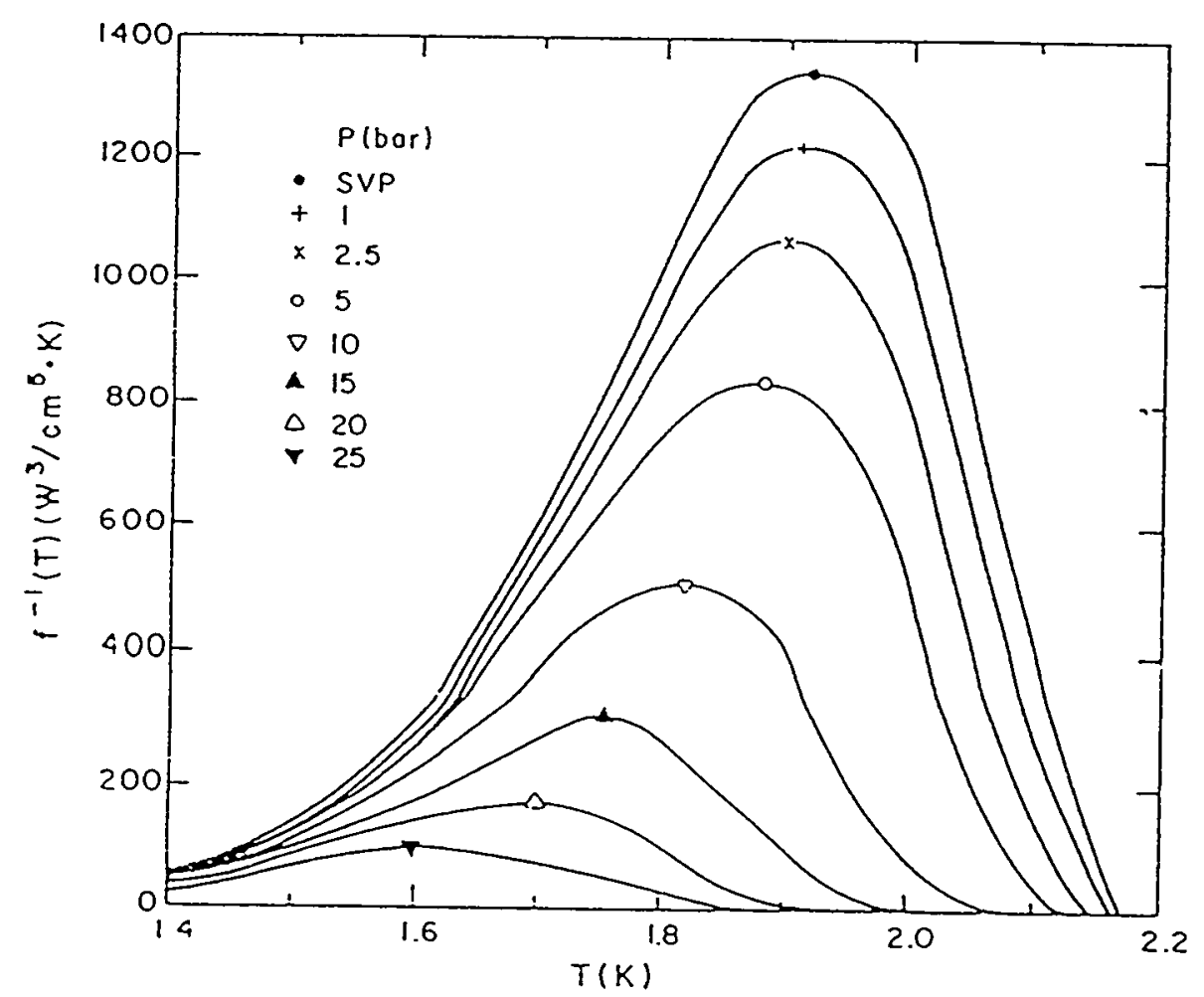

Figure 2.3. Heat conductivity function $f^{-1}(T, P) .^{3}$

\subsubsection{Transient Heat Transfer}

Transient heat transpor mechanisms in He II are important for understanding the stability of superconducting magnet systems since thermal disturbances in the magnets are principally of 
short duration. As discussed in a previous section, if the heat flux $q$ is applied to one end of a channel as shown in Figure 2.2, the temperature distribution within a channel changes as a function of time until it reaches a steady-state condition.

Transient temperature profile in He II is considered to depend on the heat transport and enthalpy. It is possible to use a heat diffusion like model to describe the transient heat transport mechanism in $\mathrm{He}$ II. According to the conservation of energy equation,

$$
\nabla q+\rho C_{p} \frac{\partial T}{\partial t}=0
$$

with a Gorter-Mellink relation, Equation (2.10), one can obtain a non-linear partial differential equation,

$$
\rho C_{p} \frac{\partial T}{\partial t}=\nabla\left[\left(\frac{1}{f(T)} \nabla T\right)^{1 / 3}\right]
$$

where $\rho$ is density and $C_{p}$ is specific heat capacity at a constant pressure.

Generally, Equation (2.14) is very difficult to solve. However, there exists a number of special cases where analytic solutions are possible. ${ }^{10}$ Further, numerous groups have developed numerical solutions to Equation (2.14) to explain specific results.

\subsubsection{Kapitza Conductance}

The heat transfer mechanism between the metal and He II is controlled by a phenomenon, known as Kapitza conductance, at the low heat flux region (without boiling of $\mathrm{He}$ II). It is necessary to understand Kapitza conductance since it is an important heat transfer mechanism in applications such as superconducting magnet systems cooled by He II. Although the Kapitza conductance is determined by experimental measurements, some theories have been 
developed to understand the phenomenon. This section reviews some of the experimental data as well as theoretical understanding of Kapitza conductance.

In 1941, Kapitzall found that a thermal boundary resistance exists at the interface between He II and a solid, when heat flux $q$ flows from the metal to the liquid. In other words, temperature difference $\Delta T_{s}$ is established between the metal and the liquid at the boundary, which is proportional to the heat flux $q$ when $q$ is small. The general expression for the Kapitza conductance is,

$$
h_{K}=\frac{q}{\Delta T_{s}}
$$

Kapitza also found that $h_{K}$ is approximately proportional to $T^{3}$ at He II temperature.

Theoretical explanation for this phenomenon was first developed by Khalatnikov. ${ }^{12}$ The idea behind his acoustic mismatch theory is that Kapitza conductance results from a large impedance to the thermal phonons which attempt to across the interface between a solid and liquid helium. The main factor which contributes to the reflection as well as transmission of phonons at the interface is the acoustic impedance of both media. The product of density and sound velocity in a solid is much larger than that of liquid helium, phonons can not travel freely across the interface because of the conservation of energy and momentum. As a result, the transfer of phonon energy is hindered which induces discontinuity in the temperature profile at the interface, whenever thermal energy is transported from one media to the adjacent media.

The heat flux that is transmitted from the solid to the liquid helium is estimated based upon finite transmission coefficient of phonons which enters the boundary and the fluid can only sustain a longitudinal component of waves. An expression for the Kapitza conductance in terms of a Debye temperature with thermal properties of liquid helium at saturated vapor pressure gives, 


$$
h_{K}=5.5 \times 10^{7}\left(\frac{T^{3}}{M \Theta_{D}^{3}}\right) \quad\left(\mathrm{kW} / \mathrm{m}^{2} \cdot \mathrm{K}\right)
$$

where $M$ is expressed as $(\mathrm{g} / \mathrm{mol}), \Theta_{D}$ is the Debye temperature.

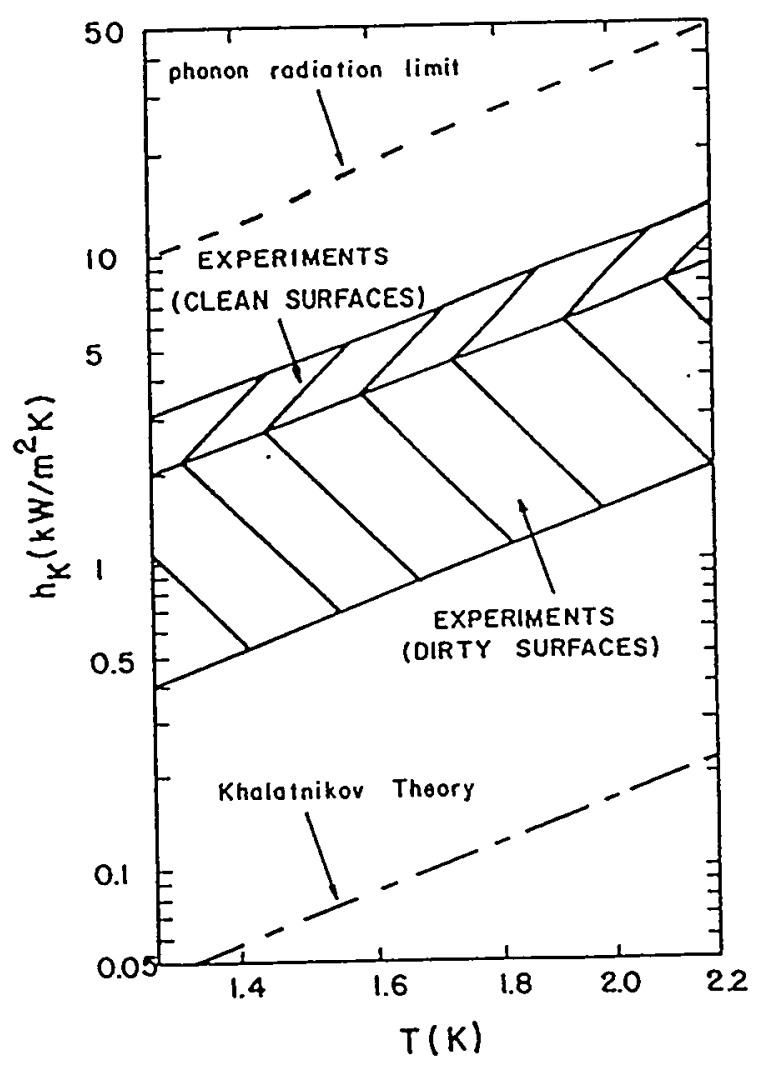

Figure 2.4 Comparison of the theory and experimental results.

Figure 2.4 shows the comparison between the Kapitza conductance calculated by the acoustic mismatch theory, the phonon radiation limit which ignored any impedance of energy transfer and experimental data of copper with temperature from $1.3 \mathrm{~K}$ to $\mathrm{T}_{\lambda}$. The Kapitza conductance of copper is most extensively investigated among solids since it has numerous technical applications at low temperature, also its high thermal conductivity gives relatively easy extrapolation of the surface temperature which contacts with He II. However, the data 
show large variation of $h_{K}$ at the same temperature of He II. The scatter of experimental data imply that the surface condition of the structure of a metal has significant effects on Kapitza conductance. The surface conditions which assumed to be affect $h_{K}$ are: roughness, crystal structure adsorption and oxidation. In general, the surface coating of the material reduce $h_{K}$. As shown in Figure 2.4, the copper with a clean surface possesses higher Kapitza conductance than that with a dirty surface.

\subsection{Supercritical Helium as a Coolant}

Low temperature helium pressurized above the critical pressure (0.226 MPa) enters the supercritical state and becomes a single phase compressible fluid. The thermodynamic properties of supercritical helium are strongly dependent on temperature and pressure, particularly around the critical point $(5.2 \mathrm{~K}, 0.226 \mathrm{MPa})$. Normally, supercritical helium is pressurized to approximately $1 \mathrm{MPa}$ when used as a coolant for a typical forced flow type superconducting magnet. This is to avoid drastic changes of the thermodynamic properties of supercritical helium.

The advantages of using supercritical helium as a coolant are as follows. First, heat transfer rates can be controlled with different flow rates, which is actually comparable or even better than that of pool boiling method. Second, there is no sudden decrease of heat transfer when the heat flux exceeds the peak nucleate boiling point. Third, flow instabilities that are caused by vapor locking of a cooling path or choke flow can be minimized. Finally, superconducting magnet can have wider operating temperature than that of a pool boiling type. Figure 2.5 shows typical heat transfer rates for low temperature helium.

This approach also has several disadvantages, the cooling paths for a superconducting magnet has to be vacuum leak tight and a pump or compressor is necessary to circulate the supercritical helium. Further, the cooling path is very long so that the pressure drop across it 
has to be taken into the consideration. Otherwise, the pressure within a cooling path may be lower than the critical pressure and this induce two-phase flow with associated instabilities and uncertainties of flow regime. ${ }^{13}$

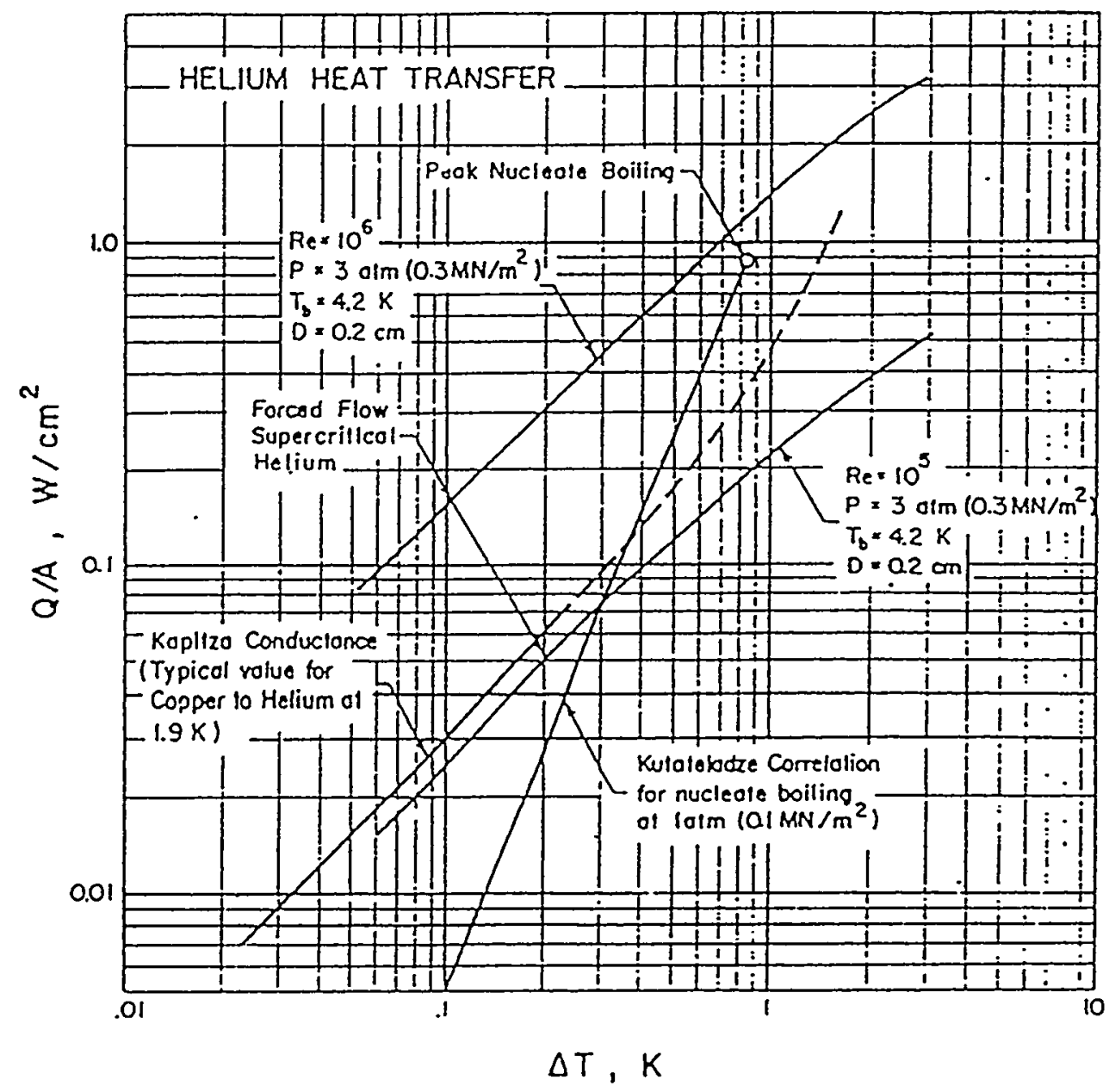

Figure 2.5. Various heat transfer rates of helium.

Therefore, it is necessary to understand the fluid mechanics as well as heat transfer mechanisms appropriate to supercritical helium as a coolant for forced-flow superconducting magnet systems. This section reviews basic fluid mechanism and two heat transfer mechanisms; forced convection heat transfer and transient heat transfer. 


\subsubsection{Fluid Mechanics}

Arp ${ }^{14}$ studied steady state supercritical helium flow in a tube, including the acceleration of the helium due to density variation along the tube. He approximated the pressure and temperature gradient along the tube for the case of nearly ideal gas and flow velocity is lower than $10 \mathrm{~m} / \mathrm{s}$. The equations that describe this process are,

$$
\begin{aligned}
& \frac{d p}{d x}=-\frac{2 f G^{2}}{\rho D}+\frac{4 q G \beta}{\rho D C_{p}} \\
& \frac{d T}{d x}=\frac{4 q}{G D C_{p}}-\frac{2 f G^{2}}{\rho D}\left(\Phi+v^{2} \frac{\kappa}{C_{p}}\right)
\end{aligned}
$$

where $G=\dot{m} / A$ and $\phi=\left(\mu_{j} C_{p}+v^{2} \kappa\right) /\left(C_{p}-v^{2} \beta\right)$. Also $\kappa$ is the bulk compressibility, $\beta$ is the bulk expansivity and $\mu_{j}$ is the Joule-Thomson coefficient.

For the forced-flow cooling systems with Reynolds number approximately $10^{5}$, the second term in Equation (2.17) dominates as heat flux becomes larger than $1 \mathrm{~W} / \mathrm{cm}^{2}$. Similarly, the second term in equation (2.18) becomes significant only if the heat flux is less than a few $\mathrm{mW} / \mathrm{cm}^{2}$.

Dean et al. ${ }^{15}$ compared Equation (2.17) and (2.18) with their experiment which supercritical helium flows in a $500 \mathrm{~m}$ long tube with a length diameter ratio $\sim 10^{5}$ as shown in Figure 2.5. The input parameters for equations are $\dot{m}=0.928 \mathrm{~g} / \mathrm{s}, D=4.8 \mathrm{~mm}, Q=0.074$ $\mathrm{W} / \mathrm{m}, P_{i n}=4.2$ bars, $T_{i n}=9 \mathrm{~K}, f=0.007$ and $R e=10^{5}$. Measured mass flow rate was $0.93 \mathrm{~g} / \mathrm{s}$.

The pressure drop and temperature gradient of supercritical helium can be described with a compressible fluid flow. Therefore, a simple compressible fluid dynamics model may give a good insight about the hydrodynamic characteristics of supercritical helium. 


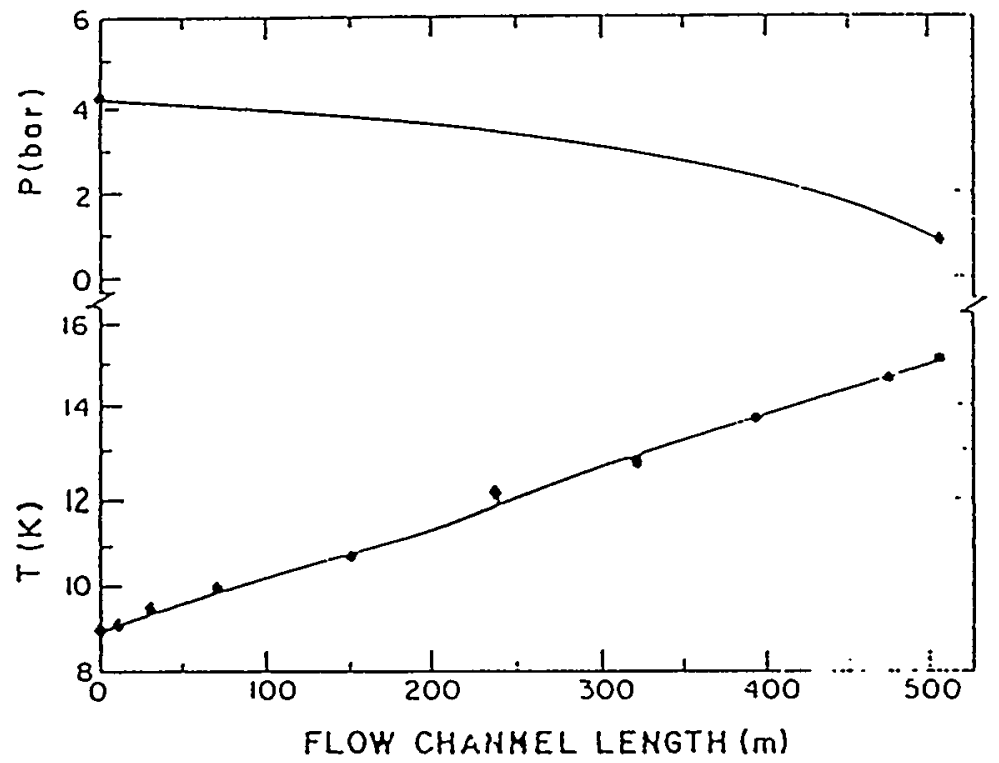

Figure 2.6 Pressure drop and temperature gradient of supercritical helium flows in a tube.

\subsubsection{Forced Convection Heat Transfer}

Forced flow convection heat transfer to supercritical helium was extensively studied by Giarratano, Arp and Smith ${ }^{16}$. They measured forced convection heat transfer coefficients over a wide range of operating conditions; Reynolds number varies from $1 \times 10^{4}$ to $3.8 \times 10^{5}$ with pressure from 3 to $20 \mathrm{~atm}$ and temperature of supercritical helium was between $4.4 \mathrm{~K}$ to $30 \mathrm{~K}$. The test section consisted of a $0.1 \mathrm{~m}$ long $\$ 2.08 \mathrm{~mm}$ stainless steel tube which is a part of a closed flow loop. Giarratano et al. successfully correlate their data with a modification of the classical constant property Dittus-Boelter correlation,

$$
N u=C_{1} \operatorname{Re}^{0.8} \operatorname{Pr}^{0.4}\left(\frac{T_{w}}{T_{b}}\right)^{C_{2}}
$$


where $\operatorname{Re}$ is a Reynolds number, $\operatorname{Pr}$ is a Prandtl number, $T_{w}$ is temperature of a stainless steel wall and $T_{b}$ is a bulk temperature. When $C_{I}=0.023$ and $C_{2}=0$, Equation (2.19) becomes a classical Dittus-Boelter correlation.

Giarratano et al. found that experimental results best fit the equation,

$$
N u=0.0259 \operatorname{Re}^{0.8} \operatorname{Pr}^{0.4}\left(\frac{T_{w}}{T_{b}}\right)^{-0.716}
$$

The standard deviation of the empirical correlation is $8.5 \%$.

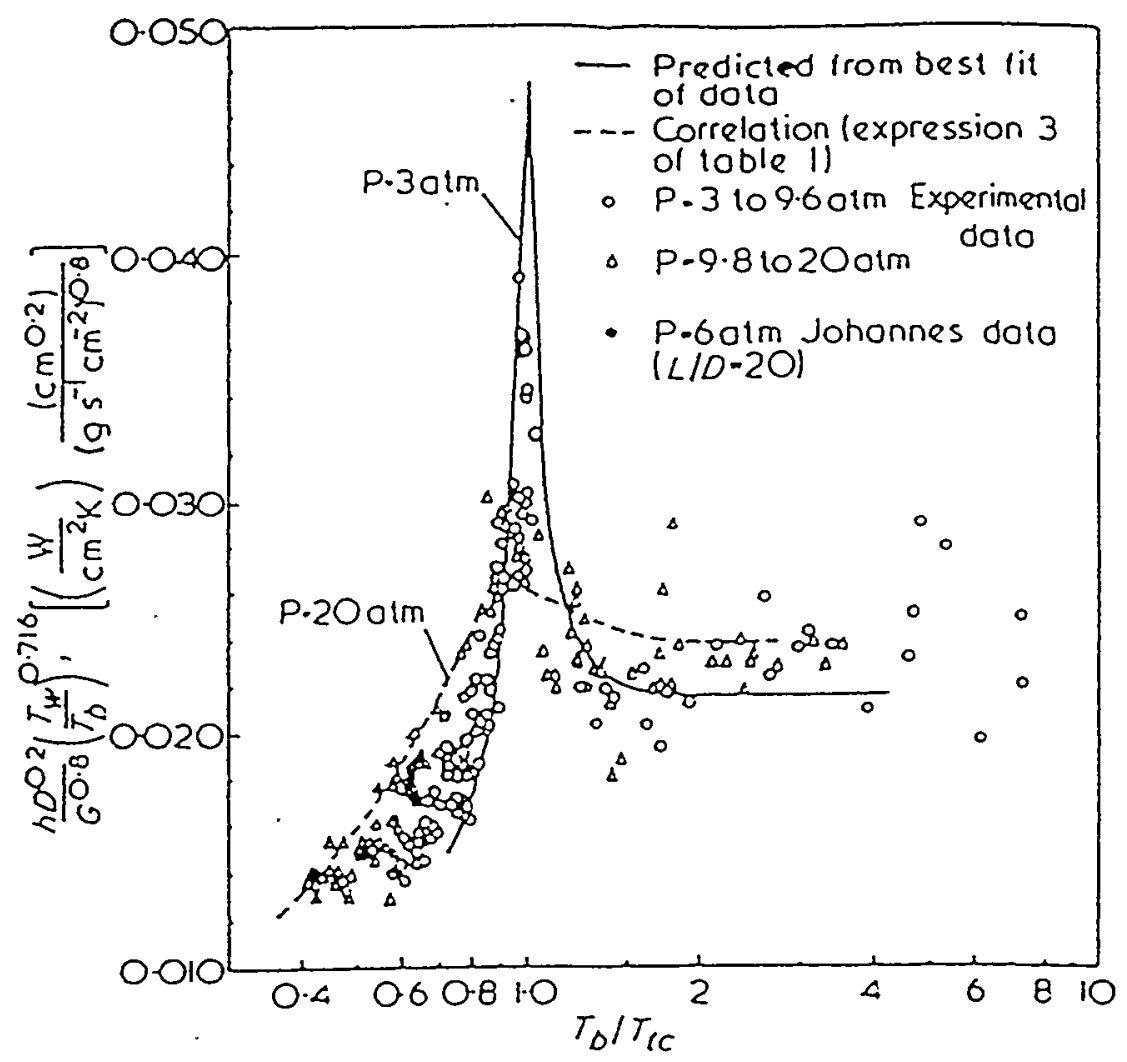

Figure 2.7. Comparison of experimental results and an empirical correlation (2.20). ${ }^{15}$

Figure 2.7 shows the experimental results as well as Equation (2.20). The vertical axis $h D^{0.2} G^{0.06}\left(T_{w} / T_{b}\right)^{0.716}$ is proportional to heat transfer coefficient and the horizontal axis is the ratio of a bulk temperature to a transposed critical temperature at which a specific heat $C_{p}$ has 
its maximum value. According to the figure, optimum heat transfer occurs near $T_{b} / T_{t c}=1$. This shows good agreement with a classical correlation which suggests that as the thermal conductivity and specific heat increase, heat transfer is improved. Consequently, forced convection heat transfer to supercritical helium is somehow related to other fluids.

\subsubsection{Transient Heat Transfer}

Transient heat transfer to both static and forced flow supercritical helium was extensively studied by Giarratano and Steward. ${ }^{17}$ The results indicate that the time to reach steady state $t_{s s}$, at which the heat transfer coefficient changes less than $3 \%$, increases with increasing applied heat flux and increasing supercritical helium temperature. On the other hand, $t_{s s}$ decreases with increasing flow rate.

Steward claimed that Kapitza resistance and development of thermal boundary layer due to the transient conduction dominates at the early stage of the heat transfer. This is the primary mechanism of heat transfer before turbulent convection effects become significant. Thus, the ratio of heat flux to the fluid to the substrate proportional to,

$$
\frac{q_{f}}{q_{s}} \propto \frac{\left.\frac{k}{\sqrt{\alpha}}\right)_{f}}{\left.\frac{k}{\sqrt{\alpha}}\right)_{s}}
$$

where $k$ is thermal conductivity and $\alpha$ is thermal diffusivity, subscript $s$ and $f$ shows the substrate and the fluid, respectively. The correlation of their experimental data was expressed by, 


$$
h_{p r e d}(t)=h_{e f f}(t)+h_{s s}
$$

where

$$
h_{e f f}=\frac{q_{f}}{T_{\text {surf }}-T_{b}}
$$

The surface temperature can be calculated in terms of Kapitza conductance,

$$
T_{\text {surf }}=T_{f . \text { surf }}+\frac{q_{f}}{h_{K}}
$$

where

$$
T_{f, \text { surf }}=T_{b}+\frac{2 q \sqrt{t}}{\sqrt{\pi}\left(\frac{k_{s}}{\sqrt{\alpha_{s .}}}+\frac{k_{f}}{\sqrt{\alpha_{f}}}\right)}
$$

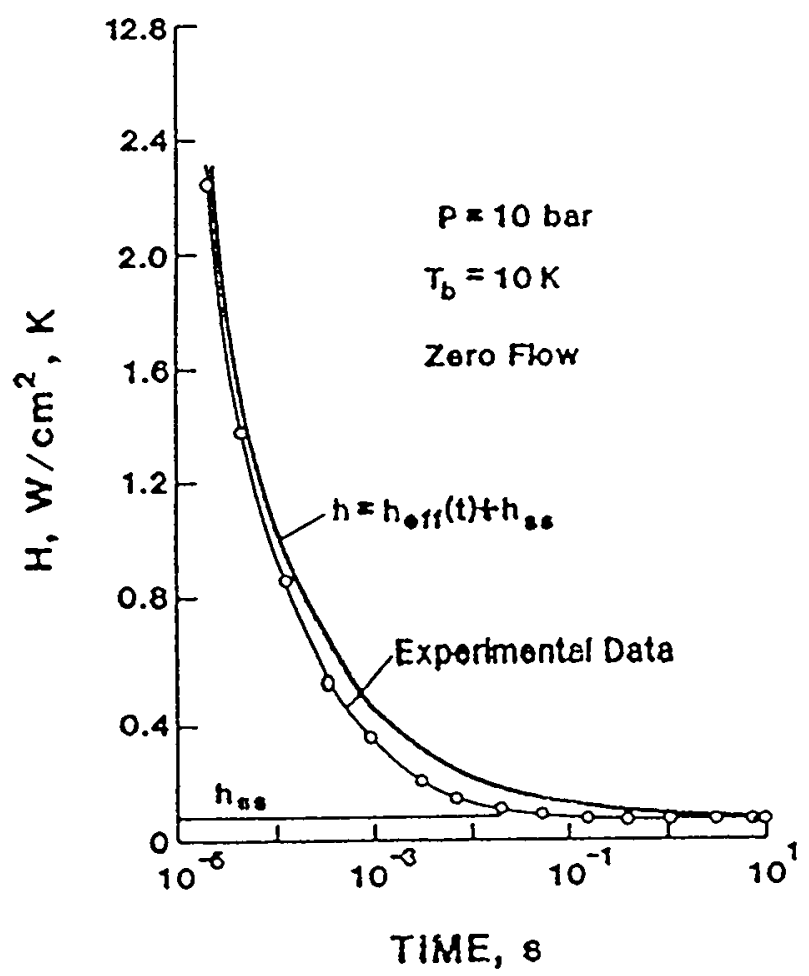

Figure 2.8. Comparison of transient heat transfer coefficient. 
As shown in Figure 2.8, the predicted heat transfer coefficient (2.22) agrees fairly well with experimental results. Note that the early stage of heat transfer, the heat transfer coefficients for both static and forced flow helium have approximately the same value. In addition, the increasing flow rate clearly reduces the time to reach the steady state, in other words, can increase the heat transfer coefficients. Although Giarratano and Steward qualitatively explained the transient heat transfer mechanism of the early stage, they did not correlate their data in terms of mass flow rates of helium.

Bloem ${ }^{18}$ also studied the transient heat transfer to a forced flow supercritical helium, using a rectangular OFHC copper tube about $155 \mathrm{~mm}$ in length and $5 \mathrm{~mm}$ hydraulic diameter. The conditions of his experiments were; inlet supercritical helium temperature was $4.2 \mathrm{~K}$, pressure between 3 and 10 bar and Reynolds number from $1.5 \times 10^{4}$ to $2 \times 10^{5}$. Heat flux up to 9800 $\mathrm{W} / \mathrm{m}^{2}$ were applied for a duration of $10 \mathrm{~ms}$ or $20 \mathrm{~ms}$.

The heat transfer coefficient was determined by;

$$
h(t)=\frac{Q_{\text {ootal }}(t)-\left.m C_{p} \frac{d T}{d t}\right|_{\text {copper }}}{A\left(T_{\text {copper }}-T_{\text {bulk }}\right)}
$$

where $Q_{\text {total }}$ is an energy applied to the heater. $T_{\text {bulk }}$ is a temperature of supercritical helium just before the heat pulse. So, Bloem assumed that the temperature of supercritical helium does not change during or after the heat pulse. The results indicate that the heat transfer coefficients do not change much with a different heat flux at the early stage of the transient heat transfer. Blocm used a transient diffusion model from a flat plate to a semi-infinite medium to describe the early stages heat transfer mechanism. According to this model, the heat transfer coefficient can be expressed as,

$$
h=\frac{1}{2}\left(\frac{\pi k \rho C_{p}}{t}\right)^{1 / 2}
$$


where $k$ is thermal conductivity, $\rho$ is density and $C_{p}$ is specific heat. All properties are that of supercritical helium at the local temperature and pressure.

The penetration depth of temperature $\delta_{T}$ to the supercritical helium in terms of conduction heat transfer can be described as,

$$
\delta_{T}=\left(\frac{4 \alpha t}{\pi}\right)^{1 / 2}
$$

where $\alpha$ is the thermal diffusivity. As time proceeds, $\delta_{T}$ increases and at a certain point $\delta_{T}$ reaches the same value as an effective layer $\delta_{e f f}$ at which turbulent heat convection dominates molecular conduction. The time to reach the transition from conduction to convection heat transfer is calculated from,

$$
t_{t}=378.6 \frac{D^{3.5}}{\dot{m}^{1.5}} \frac{\eta^{1.5}}{\alpha}
$$

Combining Equations (2.27) and (2.29), the heat transfer coefficient $h_{t t}$ at the point where conduction heat transfer dominates; may be written,

$$
h_{t t}=4.556 \times 10^{-6} \mathrm{k} \frac{\left(\frac{\dot{m}}{\eta}\right)^{0.75}}{D^{1.75}}
$$

Using Equations (2.29) and (2.30), Bloem finally derived an empirical formula for the transient heat transfer coefficient,

$$
h_{\text {sran }}=b \dot{m}^{0.75}\left(\frac{t_{t}}{t}\right)^{1 / n}
$$


where $b\left(\mathrm{~kg}^{0.25} \mathrm{~s}^{-2.25} \mathrm{~K}^{-1}\right)$ is the constant which depends on pressure and $n=500 \dot{m}+2.5$ where $\dot{m}$ in $\mathrm{kg} / \mathrm{s}$. Equation (2.31) fits the experimental data from $2 \mathrm{~ms}$ to $100 \mathrm{~ms}$ within 10 $\%$ of error. Figure 2.9 shows the experimental data as well as Equation (2.31). After $100 \mathrm{ms,}$ the heat transfer coefficient can be estimated with a modified Dittus -Boelter Equation,

$$
N u=c R e^{0.8} \operatorname{Pr}^{0.4}
$$

where $c$ is in the range 0.02 to 0.027 .

In spite of these discrepancies, the transient heat transfer is first determined by the molecular conduction with thin layer of helium, which eventually becomes dominated by turbulent convection heat transfer. Bloem recommended using high pressure supercritical helium as a coolant for superconducting magnet systems since it eliminates probability of pressure oscillations and products of density, specific heat capacity and thermal conductivity increases; that relates to the volumetric heat absorption coefficients.

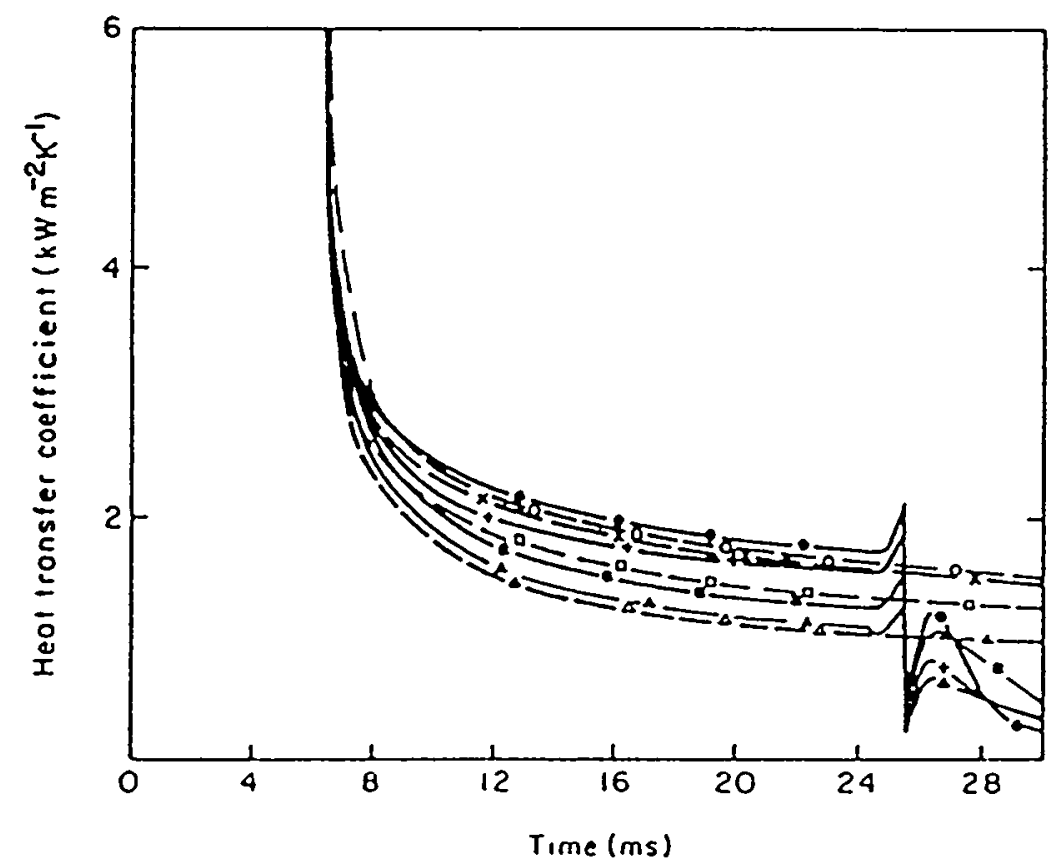

Figure 2.9. Comparison of experimental results with Equation (2.31). ${ }^{16}$ 


\section{Cable-in-Conduit Conductors (CICCs)}

The present chapter reviews the transient stability and pressure drop for CICCs. Stability issues that relate specifically to CICCs include thermally induced flow of supercritical helium and multivalued stability. Pressure drop issues are discussed with the hydraulic characterization of the conductor and some empirical fit to the friction factor variation with Reynolds number. Some numerical analyses for the new type CICCs are also discussed.

Figure 3.1 shows a cross section of the conventional Cable-in-Conduit Conductor. This type of conductor consists of the number of superconducting strands tightly encased in a stainless steel sheath with supercritical helium $\sim 4 \mathrm{~K}$ flowing through the spaces between strands. The stainless steel jacket provides a structural case and maintains the temperature of superconducting strands at their operating point. The void fraction of the conductor is typically about $40 \%$ and the strand diameter is approximately $1 \mathrm{~mm}$. Because of their mechanical strength and high overall current density, CICCs have been proposed to use in large scale superconducting magnet systems; such as the Tokamak experimental fusion reactors (ITER, TPX) and Superconducting Magnetic Energy Storage (SMES).

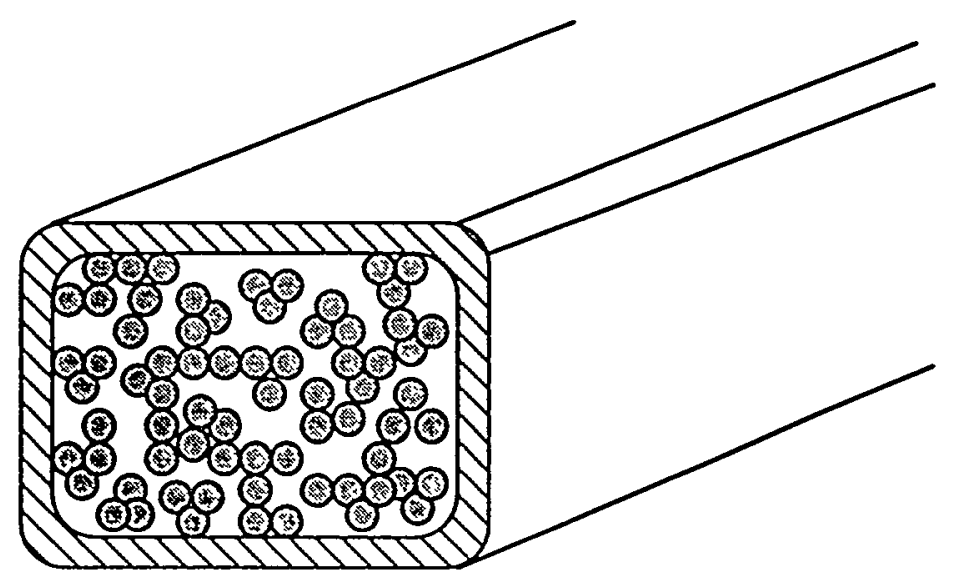

Figure 3.1. Typical cross section of a CICC. 
There are several advantages that CICCs have over pool-boiling type conductors: a continuous electrical insulation scheme; a wider operating temperature range for the superconducting magnet; and a magnet construction which provides better mechanical strength because of its distributed structure. However, CICC type superconducting magnets need more complex refrigeration systems than conventional pool-boiling type magnets and are not fully cryostable since the available enthalpy of helium is limited. ${ }^{19}$

A new type of CICC has been developed especially for the central solenoid and the toroidal field coil for ITER (International Thermonuclear Experimental Reactor) project. ${ }^{20}$ The most distinct feature is that the conductor has a hybrid cooling channel; conventional cooling to the strands and a central cooling hole as shown in Figure 3.2. In some designs, the central channels has a perforated tube or a wire/strip spiral to maintain its space through the whole windings of the magnet. The conductor consists of six bundles, each containing the same number of superconducting strands, which is wrapped with a thin stainless steel or inconel foil.

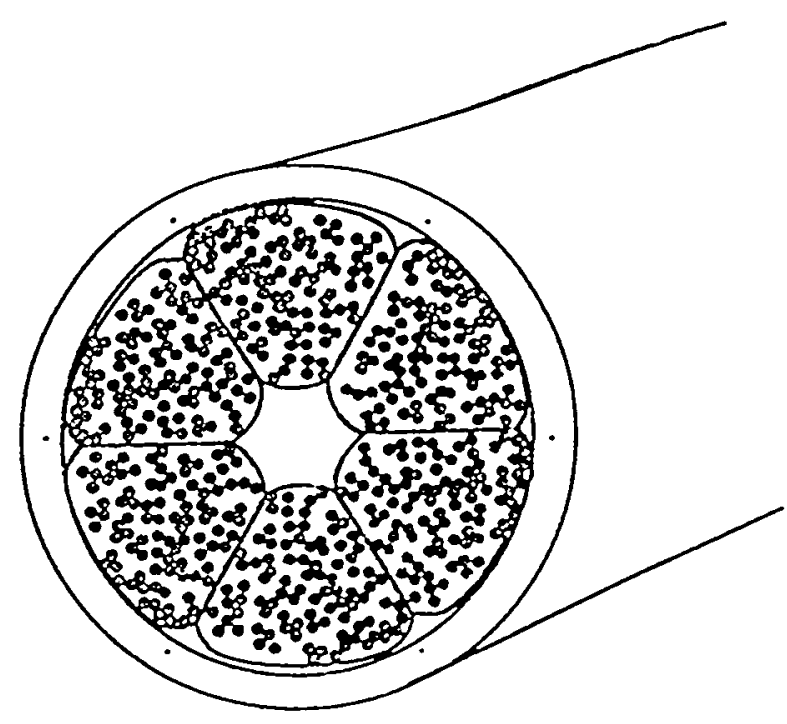

Figure 3.2. Cross section of a new type CICC. 
Typically, the foil provides $90 \%$ coverage to allow not only the helium communication between the bundle space and the central channel but also to reduce the electrical coupling among the bundles. The central channel of the conductor largely reduces the flow impedance. Therefore, a smaller overall pressure drop or an increasing mass flow rate are possible in this conductor design.

\subsection{Stability of CICCs}

In general, the stability test of a CICC is conducted by applying a thermal disturbance to a short section or entire length of a conductor to initiate a normal zone to the superconducting wire. This is accomplished by either a resistive heating or an inductive heating method. For the former method, a resistive wire is inserted within the conductor and a heat pulse is applied that increases the temperature of helium over the heated section of the conductor. However, this method distorts the geometry of the CICC, it is not a practical way to test the conductor. On the other hand, the inductive heating method uses a solenoid, which is wrapped around the conductor jacket to provide the transient magnetic field and induce eddy current to the strands. Although this technique does not modify the geometry of the CICC, a calibration of the inductive heater is necessary in order to estimate the energy input to the conductor.

The stability margin of a $\mathrm{CICC}, \Delta \mathrm{H}$, is defined as the maximum heat input at which the $\mathrm{CICC}$ can sustain or recover to its superconducting state with a given mass flow rate of the helium and the transport current. ${ }^{21}$ A number of transient stability measurements on CICCs have been reported. According to those experiments, the primary factors affecting the stability of the CICCs include: the transient heat transfer coefficient between the strands and supercritical helium; temperature and velocity of the helium; the wetted surface area and configuration of the conductor, duration and amount of the heat pulse input to the conductor. ${ }^{21}$ The overall stability of the forced-cooled magnet increases significantly as the inlet temperature 
of the helium decreases. Further the stable transport current can be increased by increasing the imposed mass flow rate of the helium. 22,23

For low helium mass flow rate, thermally induced flow is the most important factor affecting the CICC stability in supercritical helium. Once the temperature of the strands reaches the current sharing temperature, Joule heating is generated since some of the transport currents flow in a stabilizer of the strand. As a result, the helium absorbs the heat and expands longitudinally because of the local increasing the helium temperature and its pressure. This steep pressure gradient induces relatively high flow velocities, which can exceed $1 \mathrm{~m} / \mathrm{s}$. Further, the induced flow of helium assumed to develop the turbulent boundary layer that enhances the heat transfer between the strands and the helium. ${ }^{24}$

\subsubsection{Induced Flow of Supercritical Helium}

It is very important to understand thermally induced-flow of supercritical helium since it is key to providing the excellent transient stability of the conductor. An analytical solution for induced flow of supercritical helium was established by Dresner ${ }^{25}$ who used the linearized continuity and momentum equations for the compressible flow. These equations assume that induced flow velocity is much smaller than the velocity of sound and that the induced transient pressure rise is much smaller than product of density and square of the velocity of sound. The energy transfer to the helium is assumed to be in the range $\sim 50$ to $200 \mathrm{~mJ} / \mathrm{cm}^{3}$ over period of 10-20 ms, which is the typical stability margin for the CICCs. The linearized equations can be written as,

$$
\frac{\partial \rho}{\partial t}+\rho \frac{\partial u}{\partial x}+u \frac{\partial \rho}{\partial x}=0
$$




$$
\rho \frac{\partial u}{\partial t}+\rho u \frac{\partial u}{\partial x}+\frac{\partial p}{\partial x}=0
$$

where $\rho$ is density of the helium, $u$ is its flow velocity and $p$ is pressure. Similarly, the energy equation can be expressed as,

$$
\rho \frac{d}{d t}\left(\frac{1}{2} u^{2}+e\right)=-\frac{\partial}{\partial x}(\rho u)+\rho \frac{\partial q}{\partial t}
$$

where $e$ is the specific internal energy of helium, $q$ is the heat input per unit mass of helium. Linearizing equation (3.3) and combining equations (3.1) and (3.2), leads to a characteristic equation;

$$
\frac{\partial}{\partial t}\left(u \pm \frac{p}{\rho_{o} C_{o}}\right) \pm C_{o} \frac{\partial}{\partial x}\left(u \pm \frac{p}{\rho_{o} C_{o}}\right)= \pm \frac{C_{o}}{v_{o}}\left(\frac{\partial v}{\partial s}\right)_{p} \frac{1}{T} \frac{d q}{d t}
$$

where the subscript zero refers to the properties of unperturbed helium. One can see that the quantity $u \pm \frac{p}{\rho_{o} C_{o}}$ is the function of $\dot{q}$. The maximum induced velocity and pressure can be expressed as,

$$
\begin{aligned}
& u_{\max }=\frac{a_{o} l}{C_{o}} \\
& p_{\max }=\rho_{o} a_{o}\left(\frac{l}{2}\right)
\end{aligned}
$$

where

$$
a_{o} \equiv \frac{C_{o} \dot{q}}{v_{o}\left(\frac{\partial h}{\partial v_{o}}\right)_{p}}
$$


and $l$ is the heated length of a tube, $v$ is the specific volume, $h$ is specific enthalpy.

Although above equations were developed for the thermally induced flow of stagnant helium in a tube, they can also be applied to the forced flow CICC to roughly estimate the order of the thermally induced flow velocity. 26

$$
u_{\text {induced }} \approx \frac{\dot{q} P_{\text {cooled }} l}{\left(1-f_{m}\right) A_{1}} \frac{\beta}{\rho C_{p}}
$$

where $f_{m}$ is the volume fraction of the metal in the conductor, $A_{\uparrow}$ is the total area within a conduit and $\beta$ is the bulk expansivity of helium.

Shanfield et al. ${ }^{27}$ studied transient heat transfer to stagnant supercritical helium within a 2 m long OFHC copper capillary tube. 'This work simulated transient heat transfer in a CICC, using both the hydraulic characteristics of the conductor as well as the metal to the helium ratio similar to that of the conductor. As shown in Figure 3.3, the measured heat transfer coefficients were higher than the transient diffusion heat transfer coefficients,

$$
h=k(\pi \alpha t)^{-1 / 2}
$$

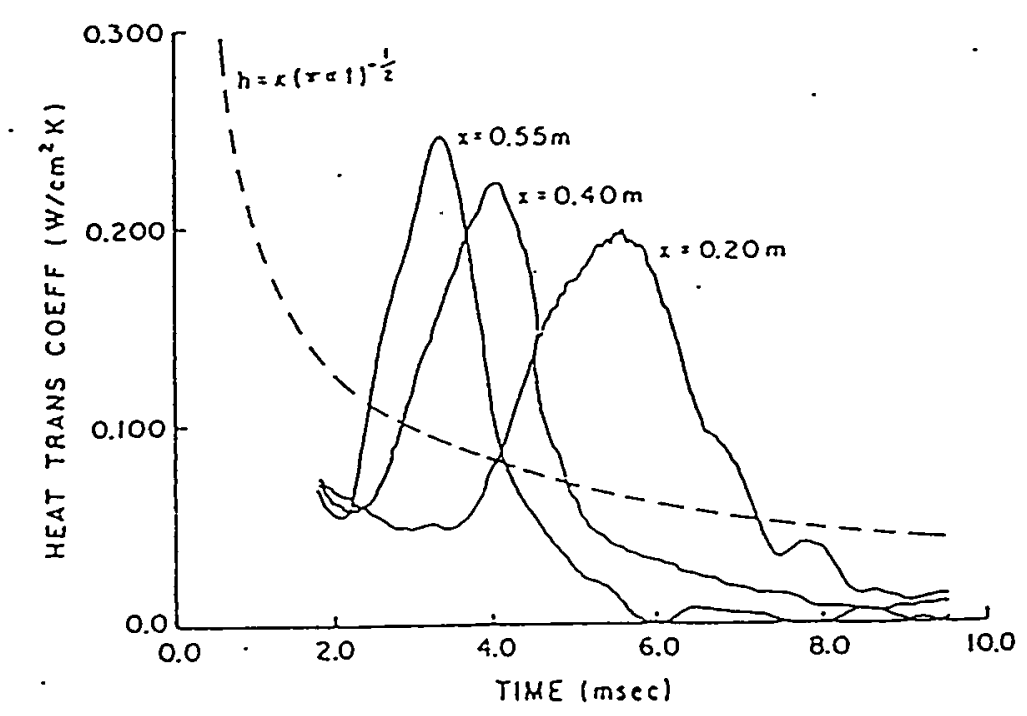

Figure 3.3. Heat transfer coefficient from Equation (3.9) and experimental results. ${ }^{27}$ 
They also compare the experimental results with numerical calculations by modifying Arp's computer code for the study of CICC appropriate to their experiment. ${ }^{28}$ This computer code simultaneously solves four partial differential equations: capillary energy balance; conservation of helium mass; momentum; and energy equations. The empirical heat transfer coefficient used for the calculation was defined as,

$$
h(x, t)=\frac{q(x, t)}{T_{w}-T_{b}}
$$

where $T_{w}$ is temperature of a capillary, $T_{b}$ is the helium temperature. The numerical calculation also shows a high heat transfer coefficient caused by the thermally induced flow of the helium, which starts at the edges of the heated section and increases rapidly with time, Figure 3.4.

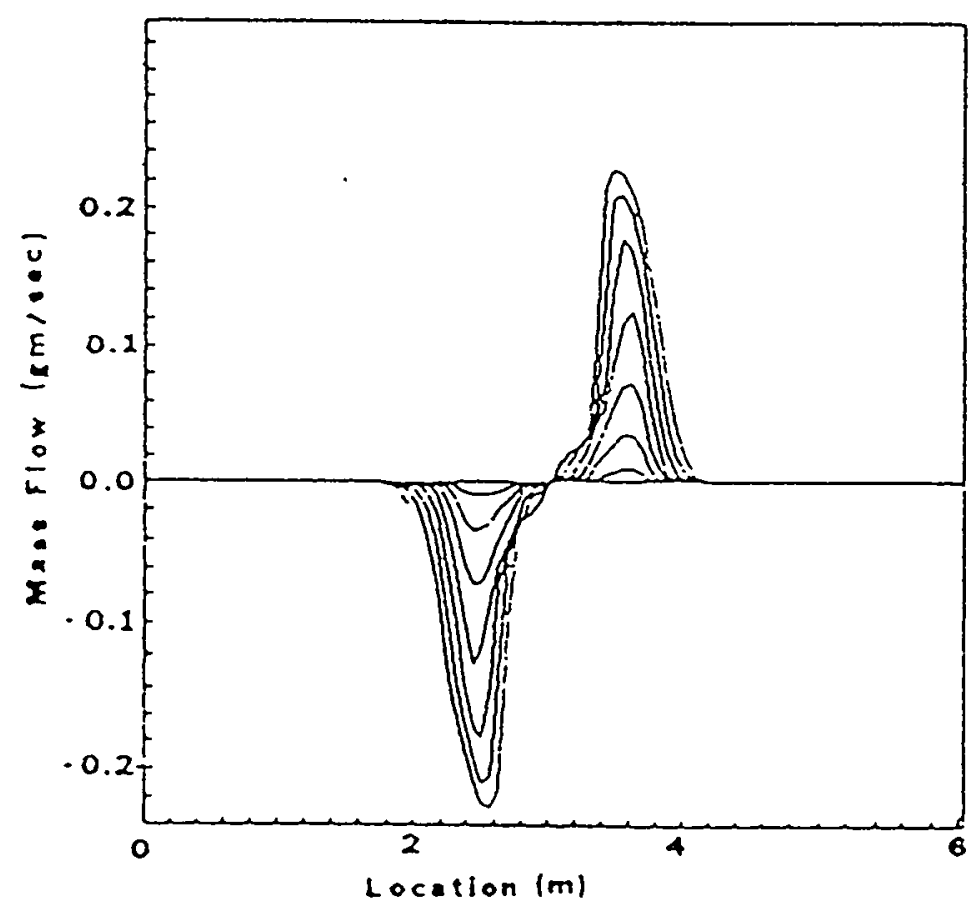

Figure 3.4. Numerical calculation of the induced mass flow rate of supercritical helium. ${ }^{27}$ 
These experimental and numerical results strongly suggest that the transient stability of CICCs is the result of thermally induced flow of supercritical helium. Other numerical and experimental results have since supported the important role of thermally-induced helium flow to the stability of CICC in the general case $\dot{m} \neq 0.29$

\subsubsection{Multivalued Stability of CICCs}

Even though CICCs show excellent thermal stability, sometimes the stability margin becomes multivalued when the helium flows slowly or is stationary. This phenomenon was first reported by Lue, Miller and Dresner ${ }^{30}$, in an experiment where the conductor was seen to be stable against small heat pulses, quenched against larger heat pulses, again recovered against even larger heat pulses and finally quenched at the largest heat pulses. Thus, the conductor has two distinct regions of stability margin: the well-cooled regime where the conductor has only the upper stability regime; and ill-cooled regime where the conductor has dual (the upper and the lower) stability margin.

Figure 3.5 shows the typical multivalued stability margin as a function of the transport current and imposed mass flow rate of the helium. The region under the surface ABEGCDF shows the recovery and above it corresponds to the quench. The transport current which corresponds at B is called the limiting current. In general, superconducting magnets are designed to operate at the transport current level which is lower than the limiting current. Therefore, it is essential to know the limiting current of the particular conductor at the early stage of a magnet design. Lue et al. suggested that the multivalued stability region ABKF is strongly dependent on a coupling between a flow-dependent heat transfer from metal to the helium and a thermally induced-flow of the helium. 


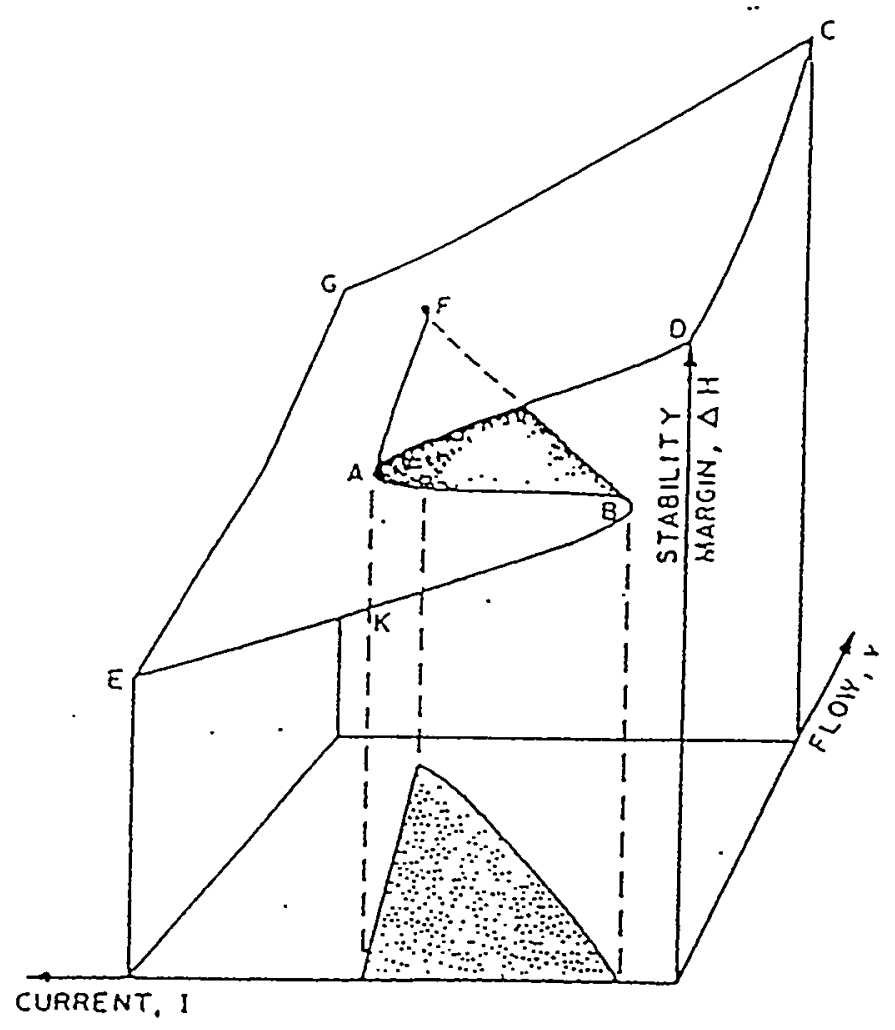

Figure 3.5. Multivalued stability curve of a CICC. 30

Dresner ${ }^{31}$ qualitatively explained the theory of multivalued stability in terms of a relationship between a flow dependent heat transfer and thermally induced flow of helium; however, the relationship is very complex since it is coupled in space and time. Dresner uncoupled the relationship and considered it as two independent phenomena. First, he assumed that the heat transfer coefficient $\mathrm{h}$ is a function of the stability margin $\Delta H$ as shown in Figure 3.6 with the constant thermophysical properties. Second, the heat transfer coefficient can be estimated by Dittus-Boelter-Giarratano correlation (equation 2.20) since the velocities of thermally induced flow of helium are of order tens of meter per second or more, which is the line OAB in Figure 
3.7. However, the correlation is basically for the steady state heat transfer. Therefore, another estimate is needed for the transient heat transfer coefficient. As discussed in section 2.3.3, the transient heat transfer coefficient is higher than that of the steady state and its mechanism is dominated by diffusion and Kapitza conductance. According to the transient heat transfer experiment by Schmidt ${ }^{32}$, the time to reach the film boiling in liquid helium is inversely proportional to the square of the interfacial heat flux. The time is related to the generation of a low density helium vapor film at the surface of the metal. Once the low density helium covers the surface, the heat transfer coefficient drops; this is corresponding to the curve $\mathrm{AB}$ of Figure 3.7. Finally, the heat transfer coefficient curve becomes curve $\mathrm{CDAB}$.

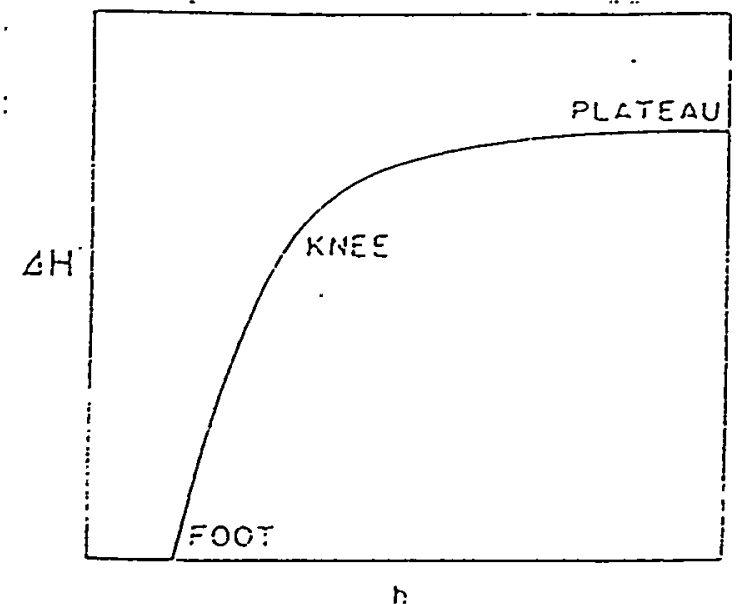

Figure 3.6. Stability curve. ${ }^{31}$

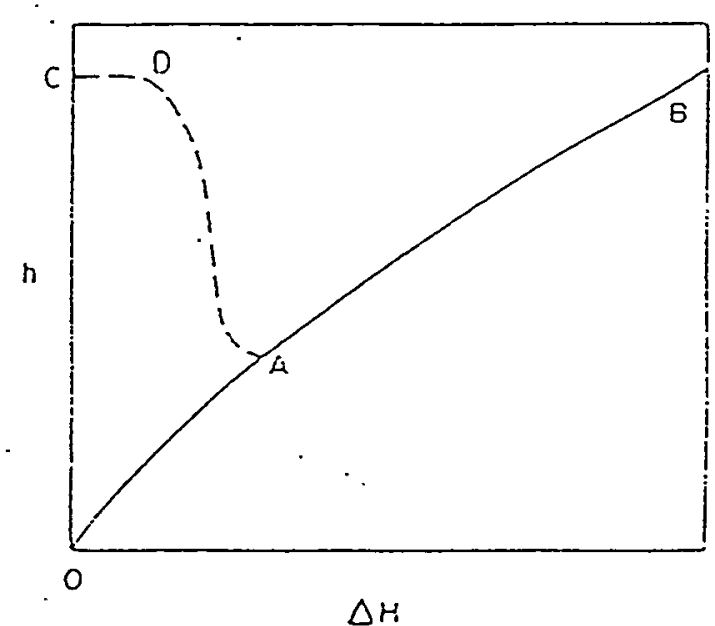

Figure 3.7. Imposed heat transfer coefficient. 31

Combining Figure 3.6 and 3.7 gives Figure 3.8. The region, at which the stability curve is lower than the heat transfer coefficient curve $\mathrm{P}<\Delta H<\mathrm{Q}$ and $\Delta \mathrm{H}>\mathrm{R}$, shows that the conductor is unstable. Thus, the origin of the multivalued stability can be understood. The transport current also affects the stability curve; the lower the transport current, the larger the area under the stability curve. In other words, the lowering the transport current provides the stability curve at $\mathrm{Q}$ and $\mathrm{R}$ becomes larger than that of the heat transfer coefficient curve. This 
corresponds to the point B of Figure 3.5 so that the conductor only has an upper band of stability.

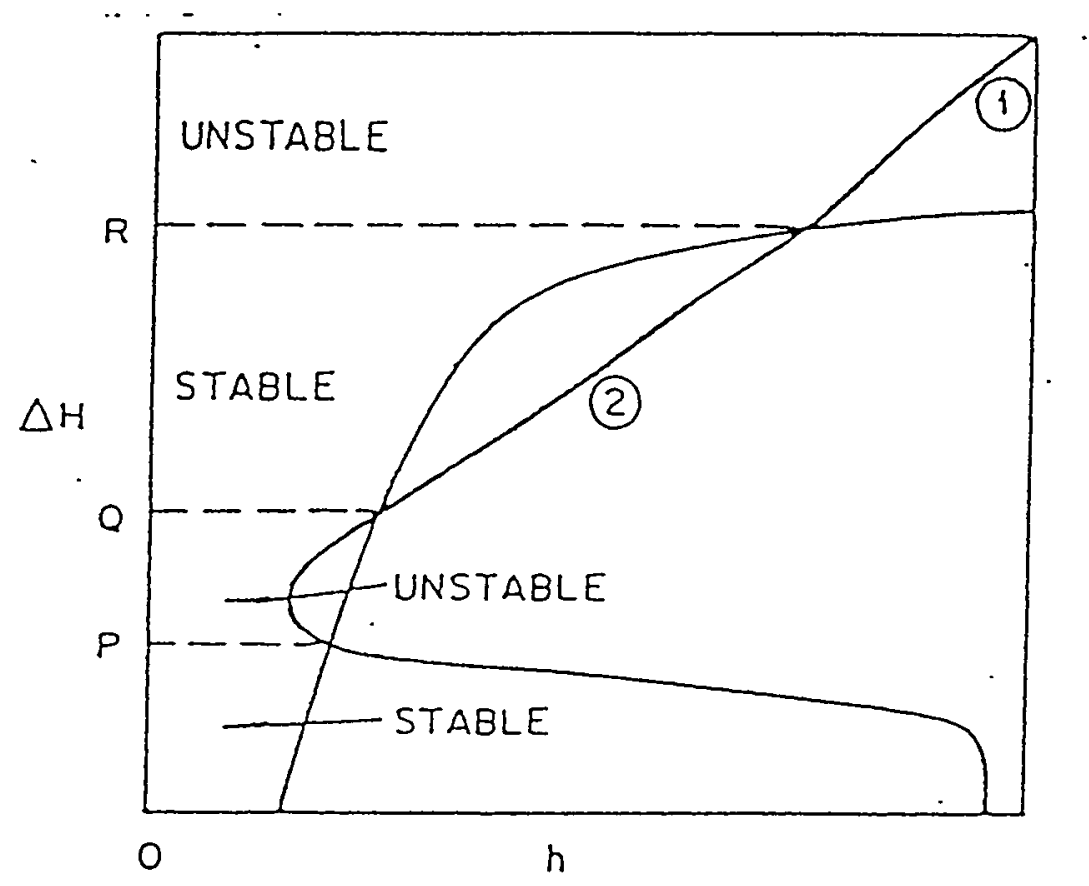

Figure 3.8. The origin of multivalued stability curve. ${ }^{31}$

\subsection{Pressure Drop in CICCs}

To design a cryogenic system for a CICC type superconducting magnet system, it is very important to estimate the pumping requirements. Certain limits exist for the operating parameters of the system such as inlet pressure and temperature of the helium, pressure drop, and mass flow rate. ${ }^{33}$ The goal of the cryogenic system is to provide an overall system that has the ability to extract the maximum heat load from the conductor with the minimum work. In general, one would expect a higher mass flow rate would absorb a higher heat load. However, as discussed in 2.3.1, frictional flow can increase the helium temperature since the 
cooling path of the system is long. In addition, the pressure drop across the total system has to be limited by the requirement that the minimum pressure of the system not drop below the critical pressure of the helium. Therefore, understanding the pressure drop associated with mass flow is important for designing CICC type superconducting magnet systems.

Lue et al. ${ }^{34}$ extensively studied the pressure drop in nine different CICCs with different conduit shapes and sizes, cable patterns, void fractions and strand surface conditions. The conduit shapes were either round or square and inner diameter of the conduit varied from 2.41 $\mathrm{mm}$ to $16.2 \mathrm{~mm}$ and square dimensions varied from $11.4 \mathrm{~mm}$ to $12.6 \mathrm{~mm}$. The void fraction was between $37.6 \%$ and $59 \%$ and the strand diameter varied from $0.73 \mathrm{~mm}$ to $1.06 \mathrm{~mm}$. The measurements were conducted with helium gas in the temperature range from $77 \mathrm{~K}$ to $300 \mathrm{~K}$, with a mass flow rate up to $4 \mathrm{~g} / \mathrm{s}$ and inlet pressure up to $1.7 \mathrm{MPa}$.

The experimental data were correlated with the following equations:

$$
\operatorname{Re}=\frac{4 \dot{m}}{\eta P_{\text {cooled }}}
$$

and

$$
f=\frac{2 \rho}{P_{\text {cooled }}} \frac{A_{\text {flow }}^{3}}{\dot{m}^{2}} \frac{\Delta P}{L}
$$

where $\dot{m}$ is the measured mass flow rate and $\eta$ is viscosity of the helium gas. $P_{\text {cooled }}$ is the cooled perimeter that is defined by an inner conduit circumference plus $5 / 6$ of a wire circumference times the number of wires. Since the cable pattern consists of a number of strand triplex, the interstices inside the triplex are considered to be inaccessible to the helium. $A_{f l o w}$ is the flow area of the helium and $\Delta P / L$ is the pressure drop per unit length of the conductor. Figure 3.9 shows a summary of the experimental results. 


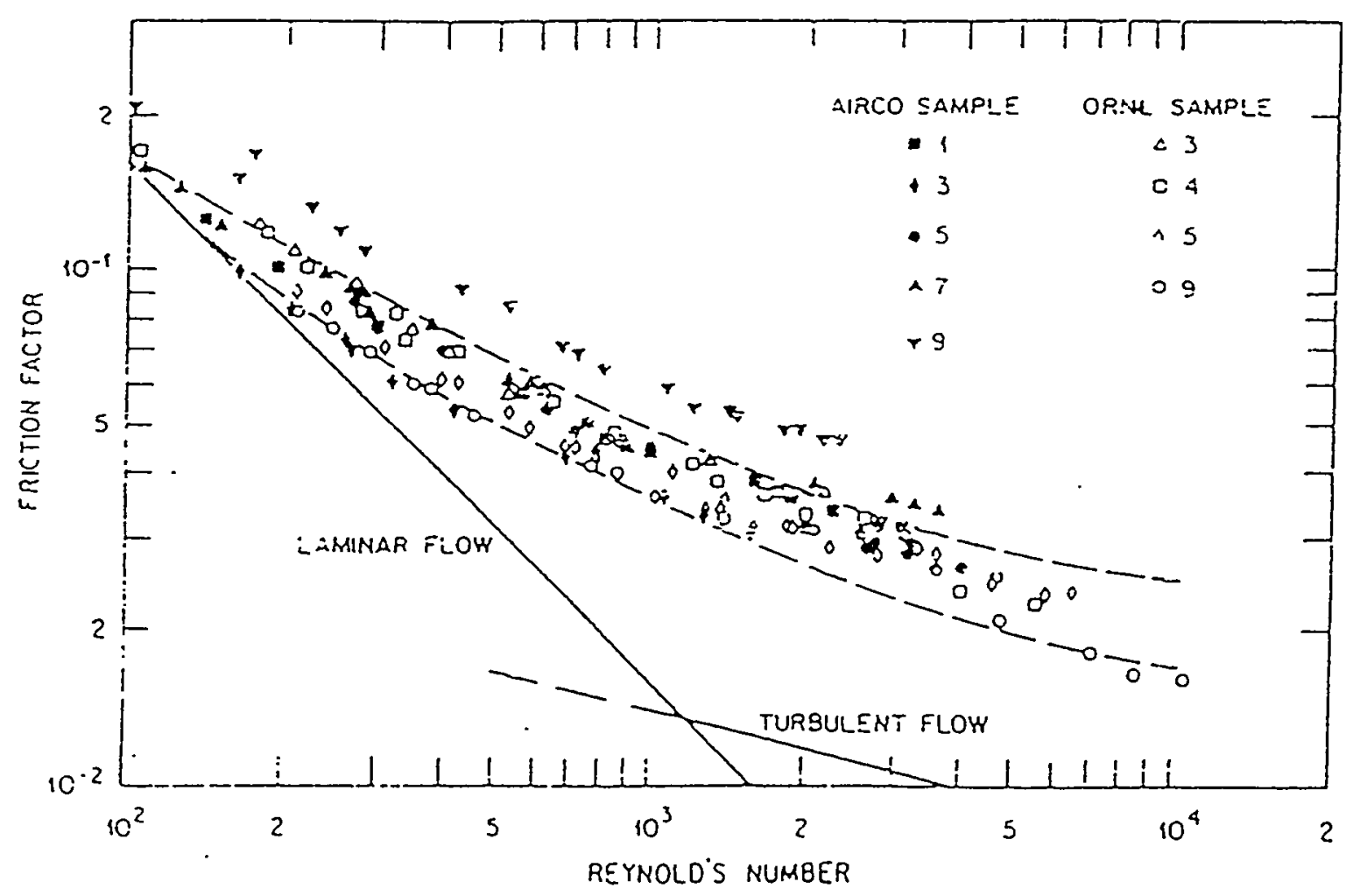

Figure 3.9. Friction factor vs. Reynolds number correlation from Lue et al.

According to Figure 3.9, all data (except Airco sample \#9) fall between the two boundary curves which shows $40 \%$ difference of the friction factor at the highest Reynolds number. However, the observed friction factor did not show any systematic trends with geometry of the conductor, such as, twist pitch, void fraction wire surface conditions or conduit shape. In addition, there is non apparent transition from laminar flow to turbulent flow.

Both Takahashi et al. ${ }^{35}$ and Shimamoto et al. ${ }^{36}$ reported the pressure drop measurements for the superconducting pulsed poloidal coil for the experimental fusion reactor in Japan Atomic Energy Research Institute (JAERI). They correlated the experimental results with an equation for turbulent flow in a tube that includes a relative roughness factor $A$. The equation can extended to fit in the low Reynolds number region. 


$$
\frac{1}{\sqrt{f}}=0.87 \ln (\operatorname{Re} \sqrt{f})-A
$$

where $\mathrm{A}$ is a numerical constant that depends on different conductors. The equation (3.4) reduces to the Nikuradze correlation for a smooth tube when $\mathrm{A}=0.8$. Takahashi et al. also found that Lue et al pressure drop measurements data can fit Equation (3.13) with $\mathrm{A}=3.0$. Other pressure drop measurements on CICCs data can be well described with Equation (3.13).

Figure 3.10 shows the variation of friction factor measurement for a Demo Poloidal Coil (DPC-TJ) which was performed by Sugimoto et al. ${ }^{37}$ in JAERI.

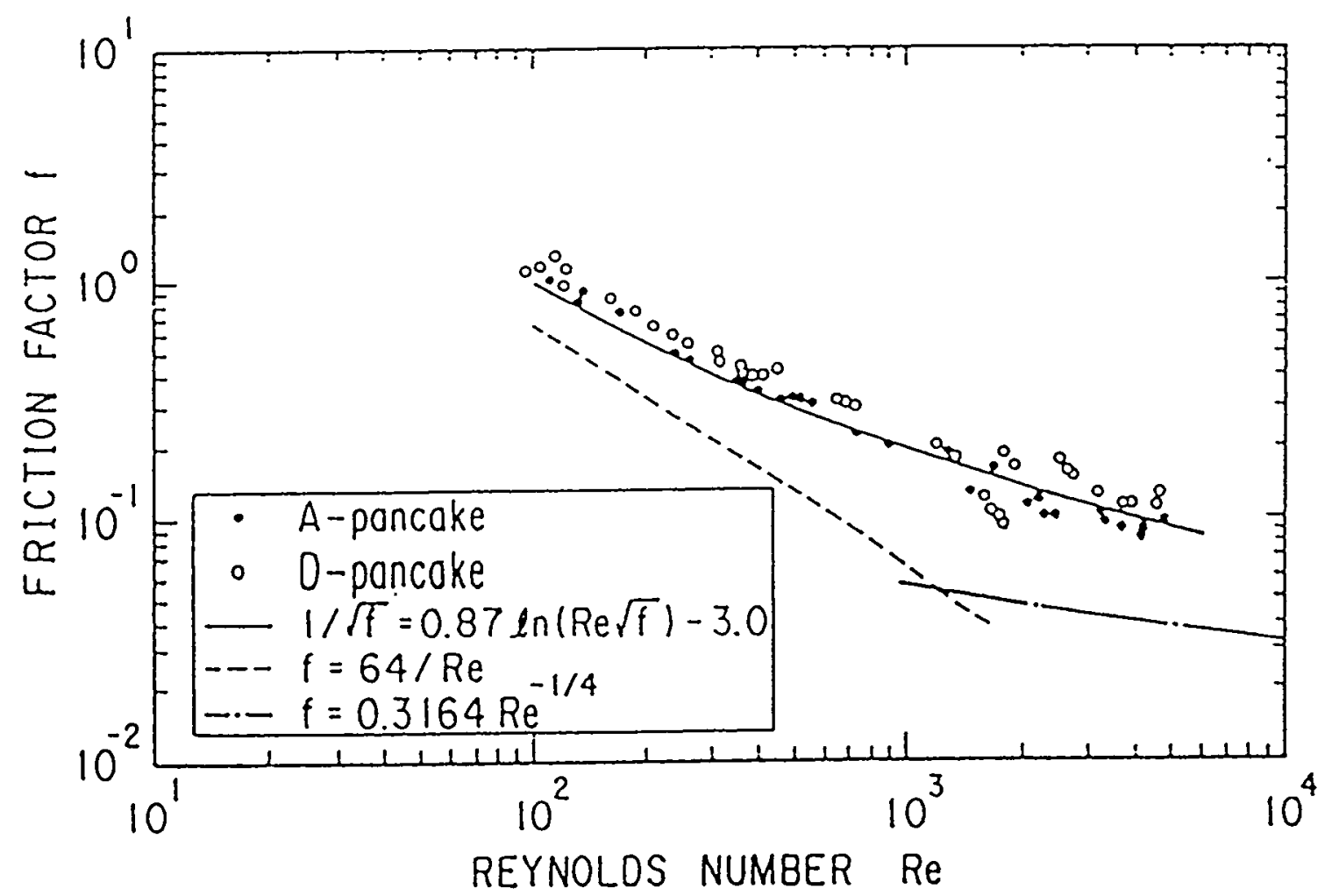

Figure 3.10. Friction factor vs. Reynolds number for the DPC-TJ. 37 
Although the relationship for friction factor versus Reynolds number for a variety of CICCs can be described with Equation (3.13), this expression does not include the information about the conductor itself: such as, void fraction, twist pitch and etc. Recently, Katheder ${ }^{38}$ derived a general correlation formula to calculate the friction factor for $\mathrm{CICCs}$ by modifying the standard formula for flow through pebble beds,

$$
f=\frac{I}{4 v_{\text {void }}^{0.72}}\left[\frac{19.5}{R e^{0.88}}+0.051\right]
$$

where $v_{\text {void }}$ is void fraction of the conductor. As shown in Figure 3.11, the formula can fit those data within a mean square error of $2 \times 10^{-3}$.

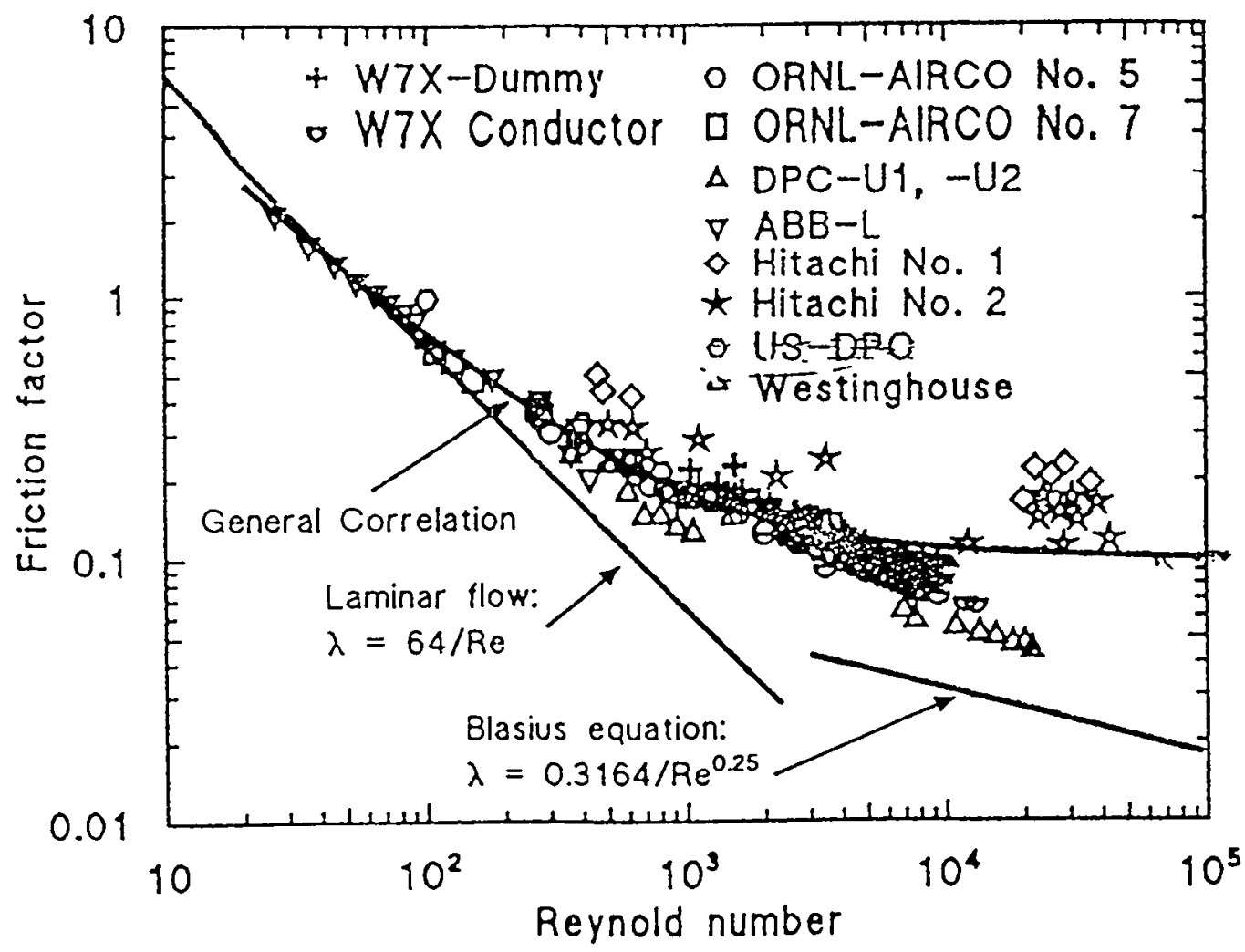

Figure 3.11. Katheder's correlation compared with experimental results. 
Daugherty 39 also reported results of the pressure drop measurements with helium temperature from $1.8 \mathrm{~K}$ to $5.7 \mathrm{~K}$ for ten different sub scale CICCs. This was the first time that He II was used as a working fluid. He did not observe clear indication of systematic trends, however, he suggested that the twist pitch of a CICC has some influence on friction factor variation; such as the longer the twist pitch, the lower the friction factor, see Figure 3.12. The temperature of the helium also influences the friction factor. An apparent transition from laminar flow to turbulent flow for two of their samples was also seen in Figure 3.13. Further, the variation of friction factor is much smaller than in a smooth tube. This may be related to the determination of cooled perimeters as well as helium flow area since they used a computer image analysis to determine those values. The associated hydraulic diameter was considerably different from that obtained by other workers.

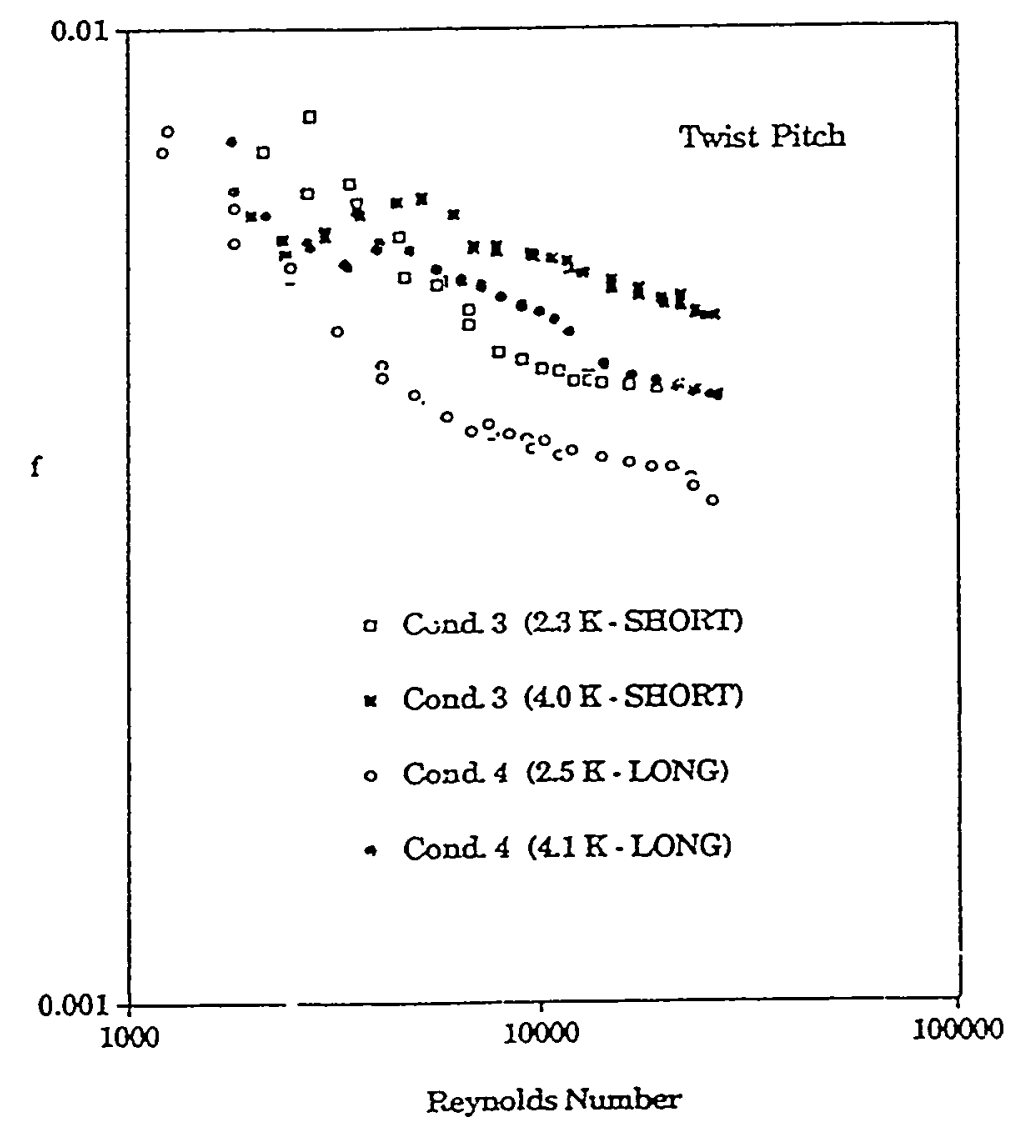

Figure 3.12. f vs. Re for the CICCs with different twist pitch. 


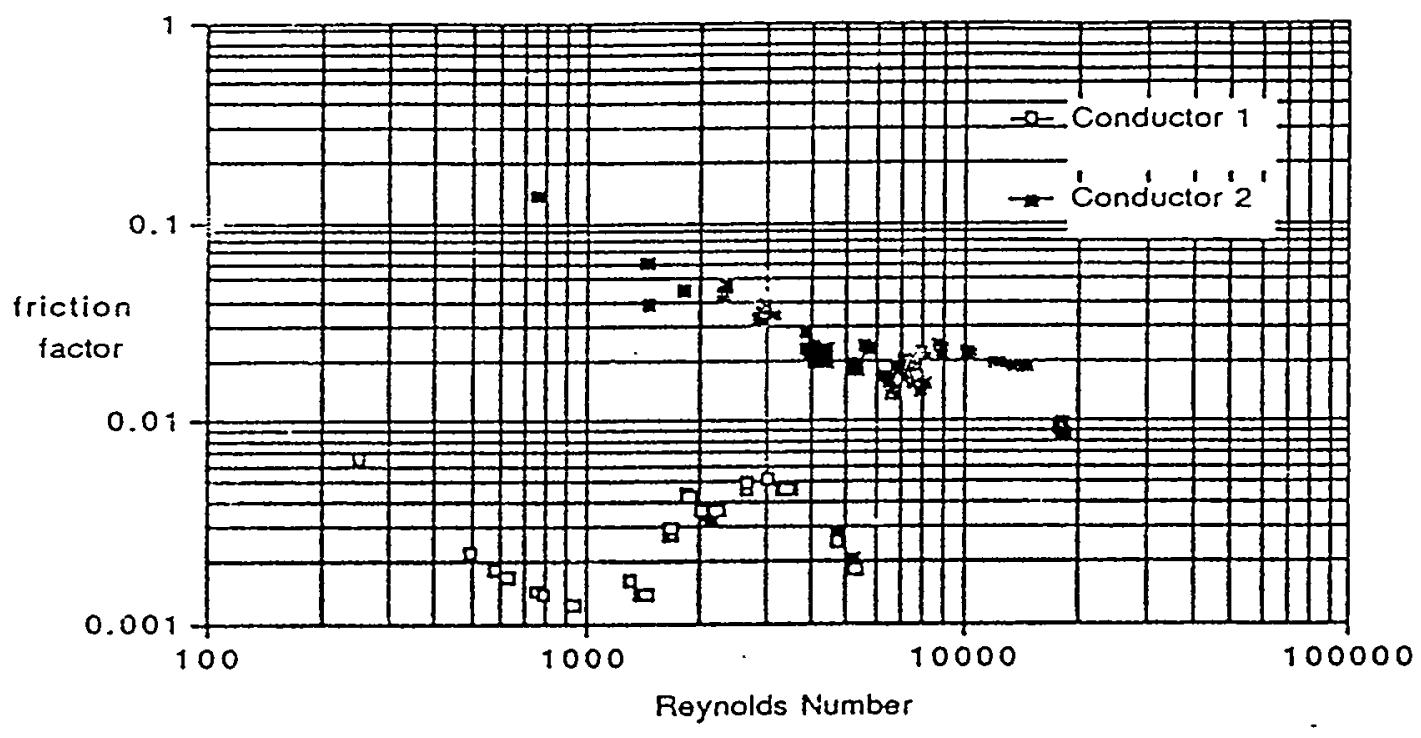

Figure 3.13 Friction factor vs. Reynolds number for CICCs with apparent flow transition. ${ }^{39}$

An interesting variation of friction factor measurements were reported by Takahata et al. ${ }^{41}$ who tested a CICC type NbTi coil for the Large Helical Device (LHD) that is a heliotron/torsatron-type experimental fusion reactor. Figure 3.14 shows the friction factor and Reynolds number measurements during the cool down of the coil, where the results were fit with Equation (3.13) with $A=3.3$. They also measured the pressure drop while charging the magnet. As the excitation number, which is defined as a charging magnet from $0 \mathrm{kA}$ to about $25 \mathrm{kA}$, increased the friction factor decreased as shown in Figure 3.15. Apparently, the Lorentz force pushes the strands to one side of the conduit, even though the strands were tightly packed ( void fraction $\sim 40 \%$ ). Consequently, a small gap appears in a conduit which significantly reduces the flow impedance of the conductor. These tendencies are clearly seen in Figure 3.16, which is the plot of friction factor versus the number of the coil excitations. It appears that the Lorentz force moves the strands.

According to the friction factor measurements of Takahata $e t$ al., the void fraction, cooled perimeter of the conductor do not represent the hydraulic characteristics of conductor. In 
addition, the small gap, which is developed in the conductor because of Lorentz forces, appears to reduce the flow impedance of the conductor.

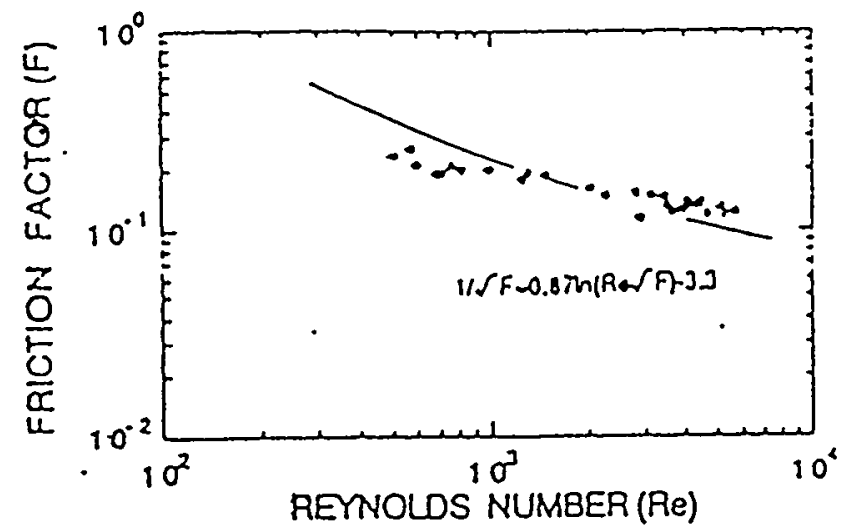

Figure 3.14. Friction factor vs. Reynolds number for Toki-PF.

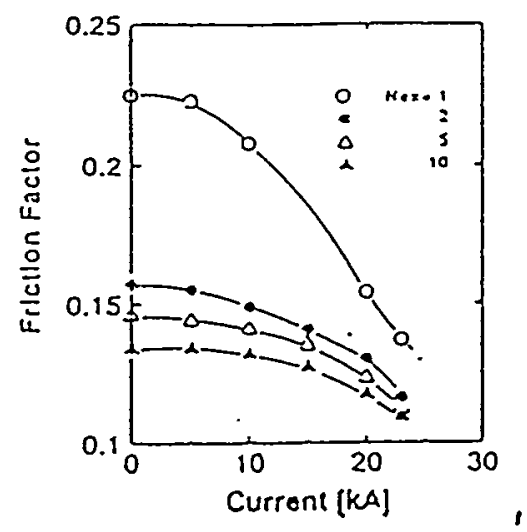

Figure 3.15 Friction factor vs. excitation current of the coil.

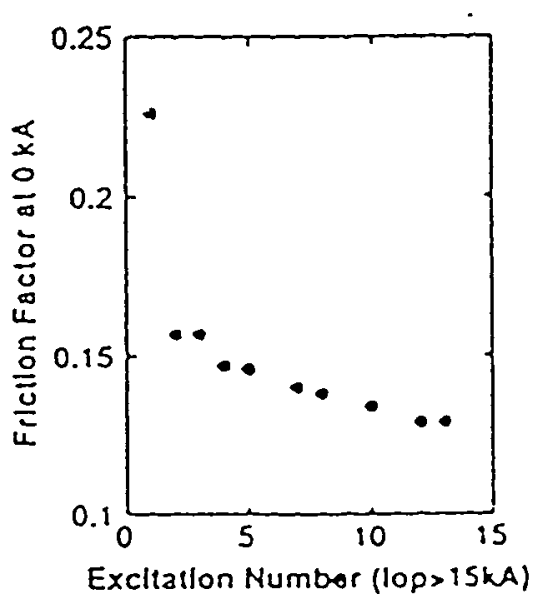

Figure 3.16. Friction factor vs. excitation number of the coil. 


\subsection{New Type Cable-in-Conduit Conductor}

Some numerical studies have been done for hybrid CICCs consisting of cabled strands surrounding a central channel. Martinez and Turk ${ }^{42}$ developed a numerical code to study thermal characteristics of the CICC with a central channel consisting of a tube. They focused on the time required to re-cool the bundle to the initial temperature after a heat pulse to the 800 $\mathrm{m}$ in length conductor. Pressure changes associated with this process were ignored. Since the hybrid CICC consists of parallel independent channels, the temperature profiles of supercritical helium in each channel strongly depends on the heat transfer between them. The effective heat transfer coefficient may be written as,

$$
\frac{l}{H}=\frac{1}{h_{b}}+\frac{e}{k}+\frac{1}{h_{c}}
$$

where $h_{b}$ is the heat transfer coefficient between supercritical helium in the bundle space and the tube, $h_{c}$ is the heat transfer coefficient between supercritical helium in the central channel and the tube and $e$ and $k$ are the wall thickness and thermal conductivity of the tube respectively.

The heat is only applied to the bundle and governing equations are,

$$
\begin{aligned}
& \frac{\partial T_{b u}}{\partial t}+U_{b u} \frac{\partial T_{b u}}{\partial x}+\beta_{b u}\left(T_{b u}-T_{c e}\right)=Q \\
& \frac{\partial T_{c e}}{\partial t}+U_{c e} \frac{\partial T_{c e}}{\partial x}-\beta_{c e}\left(T_{b u}-T_{c e}\right)=0
\end{aligned}
$$

for the bundle and central channel, respectively, where 


$$
\begin{aligned}
& \beta_{b u} \equiv \frac{H P_{c o o l e d, b u}}{\rho_{b u} C_{p, b u} A_{f l o w, b u}} \\
& \beta_{c e} \equiv \frac{H P_{c o o l e d, c e}}{\rho_{c e} C_{p, c e} A_{\text {flow }, c e}}
\end{aligned}
$$

$U$ is the velocity of the helium, subscripts bu and $c e$ refer to the parameters in the bundle and the central channel regions, respectively. The initial conditions are,

$$
\begin{aligned}
& \text { at } t=0, T_{b u}=T_{c e} \text { and } Q=\frac{q}{\rho_{b u} C_{p, b u}} \\
& \text { for } t>0, T_{b u}=T_{c e}=T_{o} \text { at } x=0
\end{aligned}
$$

where $T_{o}$ is the unperturbed helium temperature.

Equations (3.16) and (3.17) were solved with various heat transfer coefficients $H$ and the helium velocity in the bundle. According to the results, the authors defined the 'Train Effect' which is the trailing of the helium temperature profile as a function of time, see Figure 3.16. The results indicate the increasing $H$ and $U_{b u}$ reduces the Train Effect $\Delta t$. As Train Effect becomes significant, the warm helium stays in the conductor for longer periods of time. Consequently, the effect deteriorates the cooling effect of the conductor which will increase the possibility to quench the magnet. They concluded that either the thickness of wall of the tube has to be thin enough or the conductor should not have a tube to obtain maximum heat transfer between two cooling channels and it reduces the Train Effect $\Delta t$. 


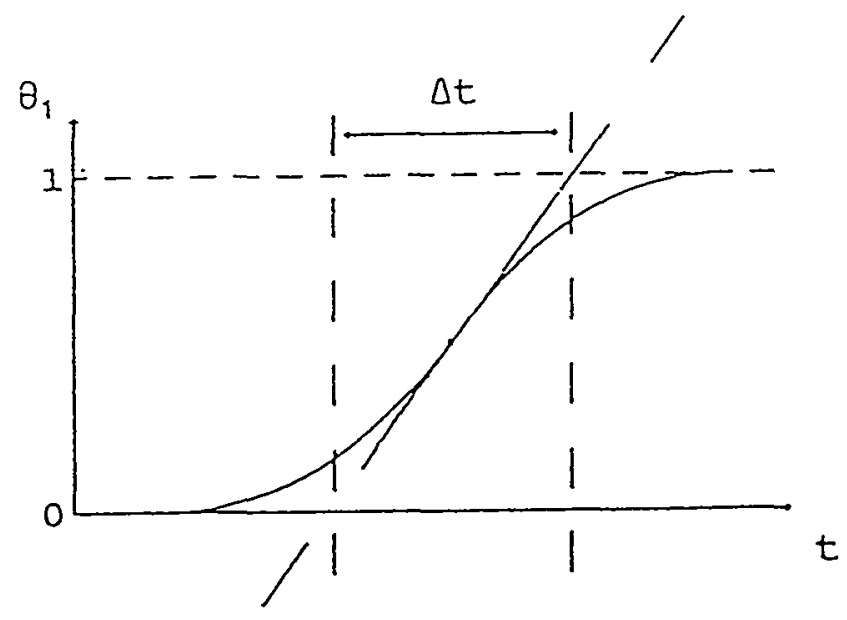

Figure 3.16. Train Effect $\Delta t$, normalized temperature profile as a function of time.

Bottura $^{43}$ developed the numerical code which describes the thermal hydraulic behavior of supercritical helium in a central channel and in a bundle space for a central solenoid of ITER (ITER-CS): one layer length is approximately $800 \mathrm{~m}$. The mass, momentum and energy balance equations for each channel were solved with coupling equations which describe communication of supercritical helium in the bundle with the central channel.

A code assumes that the flow is one dimensional and there are no pressure or temperature differences in transverse direction of the conductor. Further, momentum transfer between the channels is neglected. Since each channel has distinct geometry, different turbulent correlations were used for each channel. The code was used to simulate the quench of the central solenoid and found that the propagation of a normal front velocity is about the same as the average helium velocity. The fraction increases of induced mass flow rate is the same for both channels as shown in Figure 3.17.

Since the flow impedance of the central channel is much smaller than the bundle regime, one would expect the induced mass flow rate to be higher in the central channel than in the bundle space. Experimental verifications are necessary to confirm these results. 


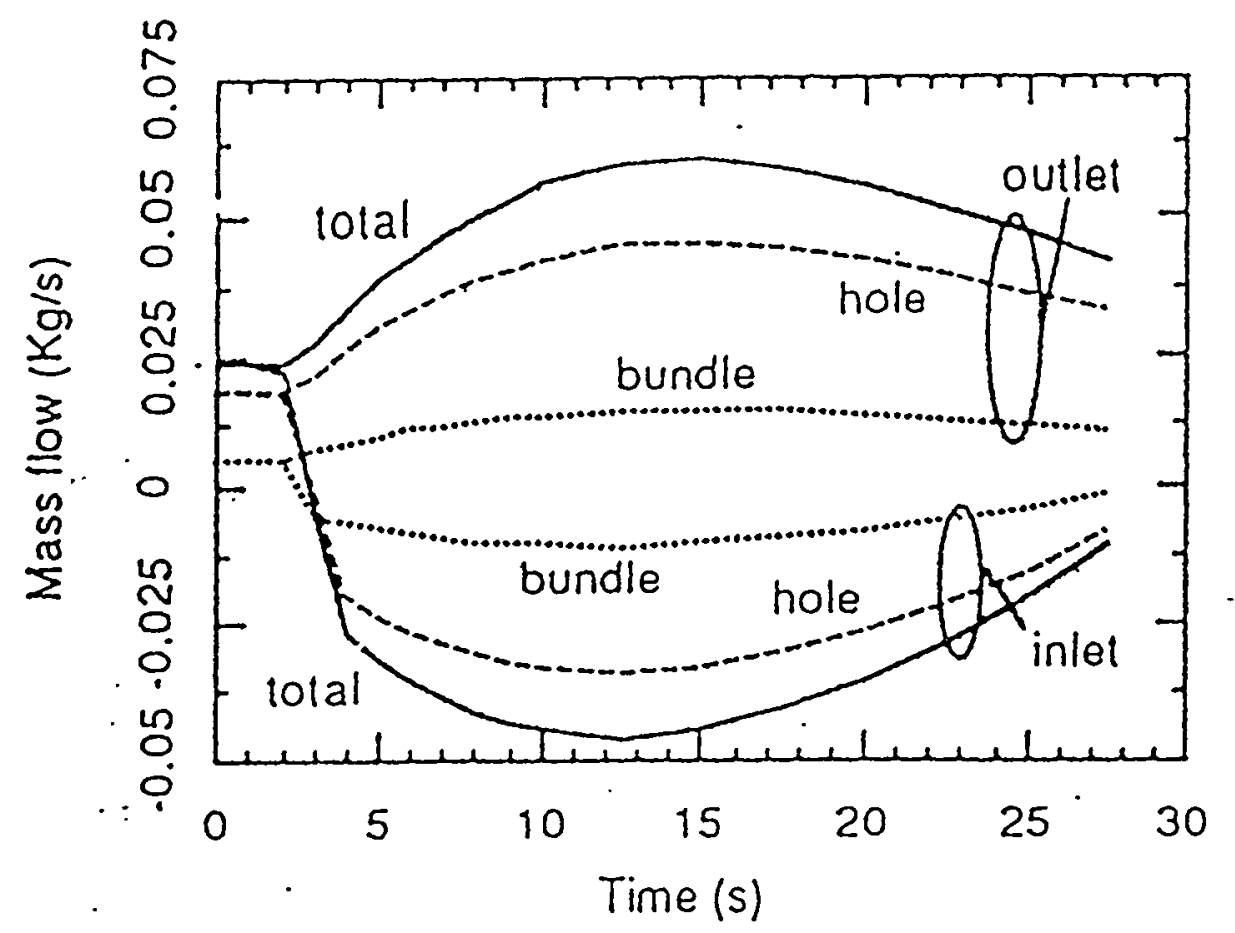

Figure 3.17. Mass flow rate vs. time after the quench.

Although many studies have been done with conventional CICCs, characterizing the variation of friction factor are still unclear. The study of some factors affecting the friction factor are necessary to obtain the hydraulic characteristics of CICCs. For the new type CICCs, experimental data are necessary to study thermal hydraulic characteristics. The variation of friction factor is especially interesting since the conductors are developed to reduce the flow impedance (overall pressure drop). Further, the distribution of supercritical helium velocity in the conductor provides us with an understanding of the communication between the supercritical helium in the bundle and the central channel regime. Characterizing the thermallyinduced flow in the conductor also provides more effective modeling of heat transfer mechanisms, which can confirm or modify some of the existing numerical models. 


\section{Description of Experiments}

Four different experiments were conducted to study thermal-hydraulic characteristics of two prototype cable-in-conduit conductors (CICCs): 1) a counterflow heat transport experiment; 2) pressure drop measurements; 3 ) inductive heater transient pressure rise experiment and; 4) transient heat transfer experiments. The present chapter describes the experimental apparatus used in this work and the corresponding procedures. The data acquisition system for the experiment is also discussed in detail. Temperature and pressure sensors, which were used to calculate the thermophysical properties of the helium and/or flow measurements, are described with their calibration procedures.

\subsection{Experimental Apparatus}

\subsubsection{Liquid Helium Flow Facility}

The Liquid Helium Flow Facility (LHFF) is a versatile experimental apparatus to study fluid dynamics as well as heat transfer mechanisms of low temperature helium. As shown in Figure 4.1, the LHFF consists of an approximately $5 \mathrm{~m}$ long, $0.2 \mathrm{~m}$ diameter horizontal cryostat which connects with two end boxes. Two vertical cryostats, $0.91 \mathrm{~m}$ long and $0.43 \mathrm{~m}$ diameter, are also mounted at each end box. In addition, a centrifugal liquid helium pump and a heat exchanger occupy each vertical cryostat, respectively. There are two access ports for each end box to install the test section inside the horizontal cryostat.

The LHFF has two radiation shields, an inner shield and a outer shield, to minimize the radiation heat leak from room temperature. The inner shield temperature is maintained around $14 \mathrm{~K}$ by a refrigerated forced flow of low temperature helium gas. Circulation of the helium 


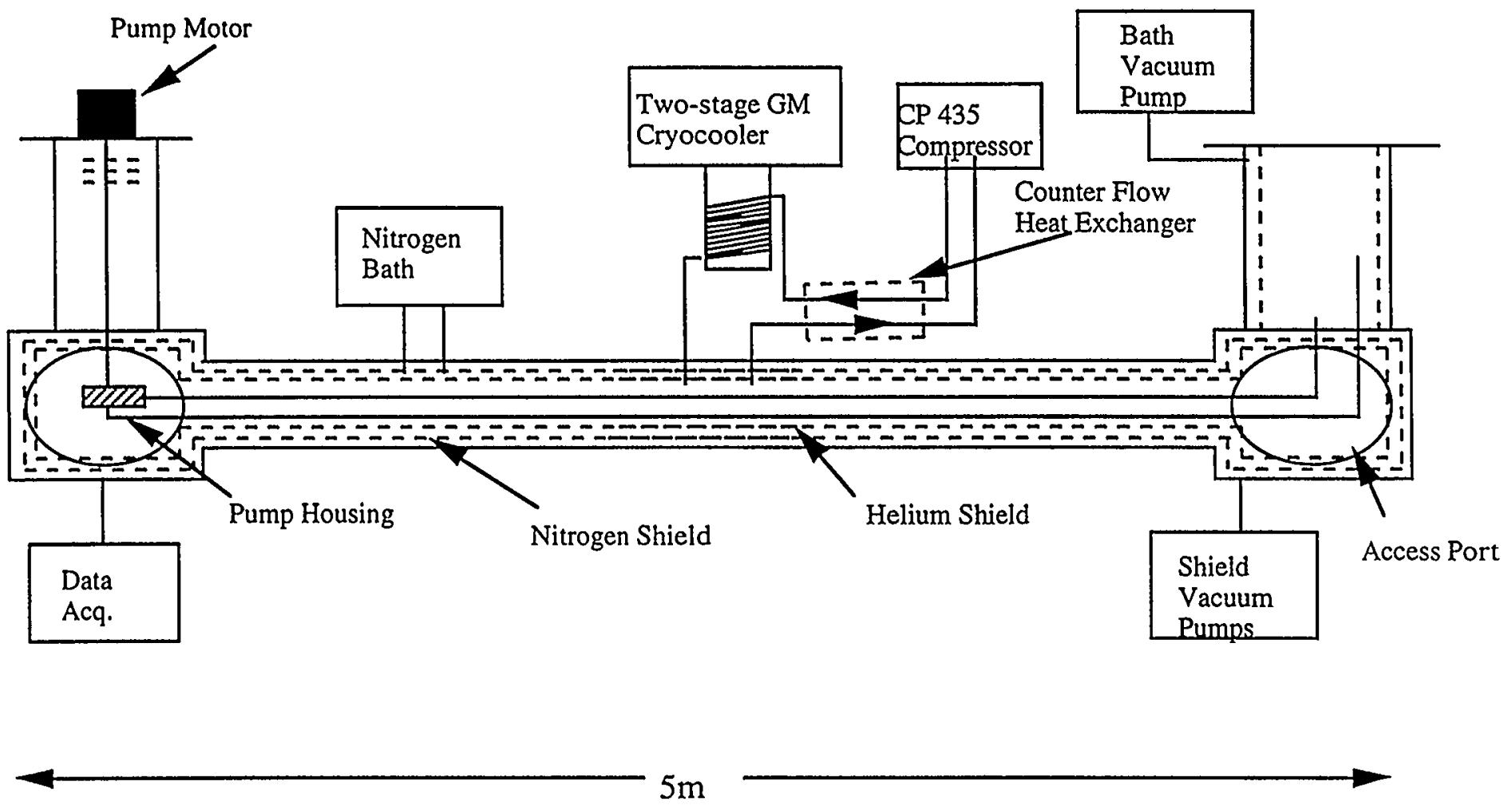

Figure 4.1 The Liquid Helium Flow Facility 
gas is achieved with a CP 435 compressor system. The helium gas is cooled with a Cryomech GB 37 cryorefrigerator/CP 25 compressor system which has a refrigeration capacity of $30 \mathrm{~W}$ at $20 \mathrm{~K}$. The temperature of the outer shield is maintained about $100 \mathrm{~K}$ during the experiment by a natural circulation loop containing liquid nitrogen.

There are two independent vacuum spaces; an inner space and a outer space, to prevent conduction heat leak from room temperature. The inner space is evacuated to $10^{-6}$ Torr by a Blazers TPS turbo-molecular pump which has a capacity of $50 \mathrm{l} / \mathrm{s}$, while the outer space is evacuated to $10^{-7}$ Torr by a Edwards Cryo-cooled Diffstak MK 2 series Model 100/300 ( a water cooled diffusion pump with a liquid nitrogen cold trap ) backed with a rotary vacuum pump Model E2M5. Two spaces are thermally isolated in an aluminum alloy housing with flanges which are connected via indium o-ring seals.

The centrifugal liquid helium pump produces a forced flow environment with mass flow rate up to $100 \mathrm{~g} / \mathrm{s}$. The pump head consists of a $68.8 \mathrm{~mm}$ diameter impeller with six straight blades in a stainless steel housing as shown in Figure 4.2.

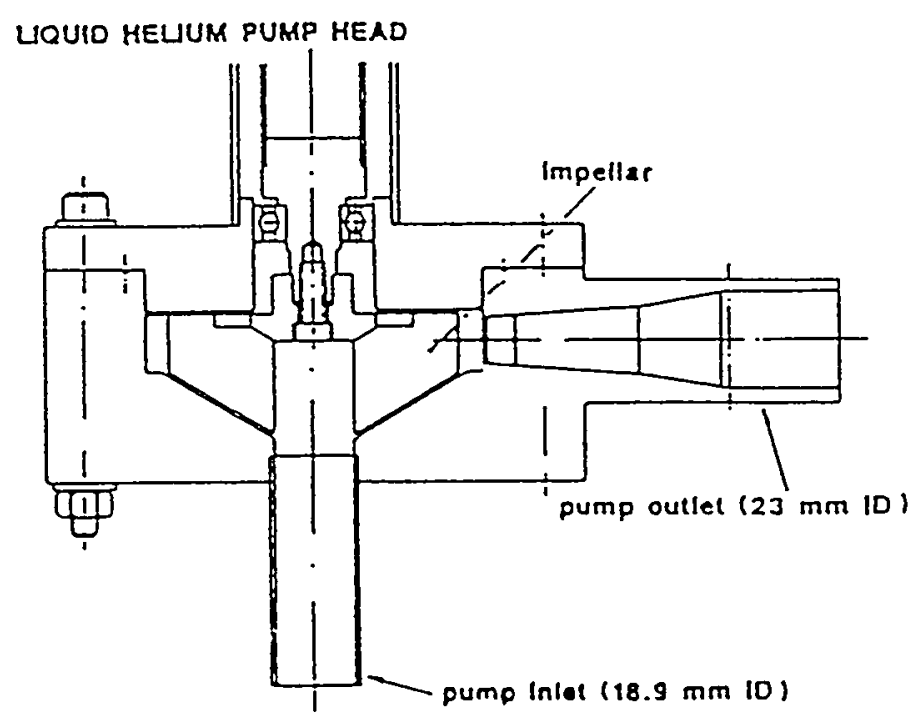

Figure 4.2. A centrifugal liquid helium pump head 
The variable speed motor, located at room temperature, is connected to the impeller through a $1.14 \mathrm{~m}$ long, $51 \mathrm{~mm}$ diameter tubular shaft that is supported by gas-lubricated ball bearings. The rotation of the motor can be varied from $0 \mathrm{~Hz}$ to $70 \mathrm{~Hz}$. To minimize the heat leak from room temperature to liquid helium environment, the tubular shaft is located inside a separate liquid helium reservoir.

The liquid heat exchanger was developed in 1989 by Weisend, Huang and Van Sciver. ${ }^{44}$ A brief description of this heat exchanger follows. As shown in Figure 4.3, it consists of a 2 $\mathrm{m}$ long, $22.2 \mathrm{~mm}$ outer diameter copper tube which is coiled and immersed in a liquid helium bath. The loop transfer control valve allows an initial liquid helium transfer to the helium bath through the test loop and the centrifugal pump. A bath control valve connects or isolates the test loop from the bath. Low temperature helium pressure in the loop can be controlled by a pressurization line which is connected to a helium gas cylinder with a pressure regulator. The pressurization line is immersed in a liquid nitrogen cold trap to eliminate any impurities in the helium gas, otherwise it may induce a blockage of the test loop. The helium bath temperature is controlled by regulating the bath pressure through a pumping port. An automatic control valve, located between the pumping port and vacuum pump, is controlled by MKS Type 651 pressure controller for the bath pressure regulation. 


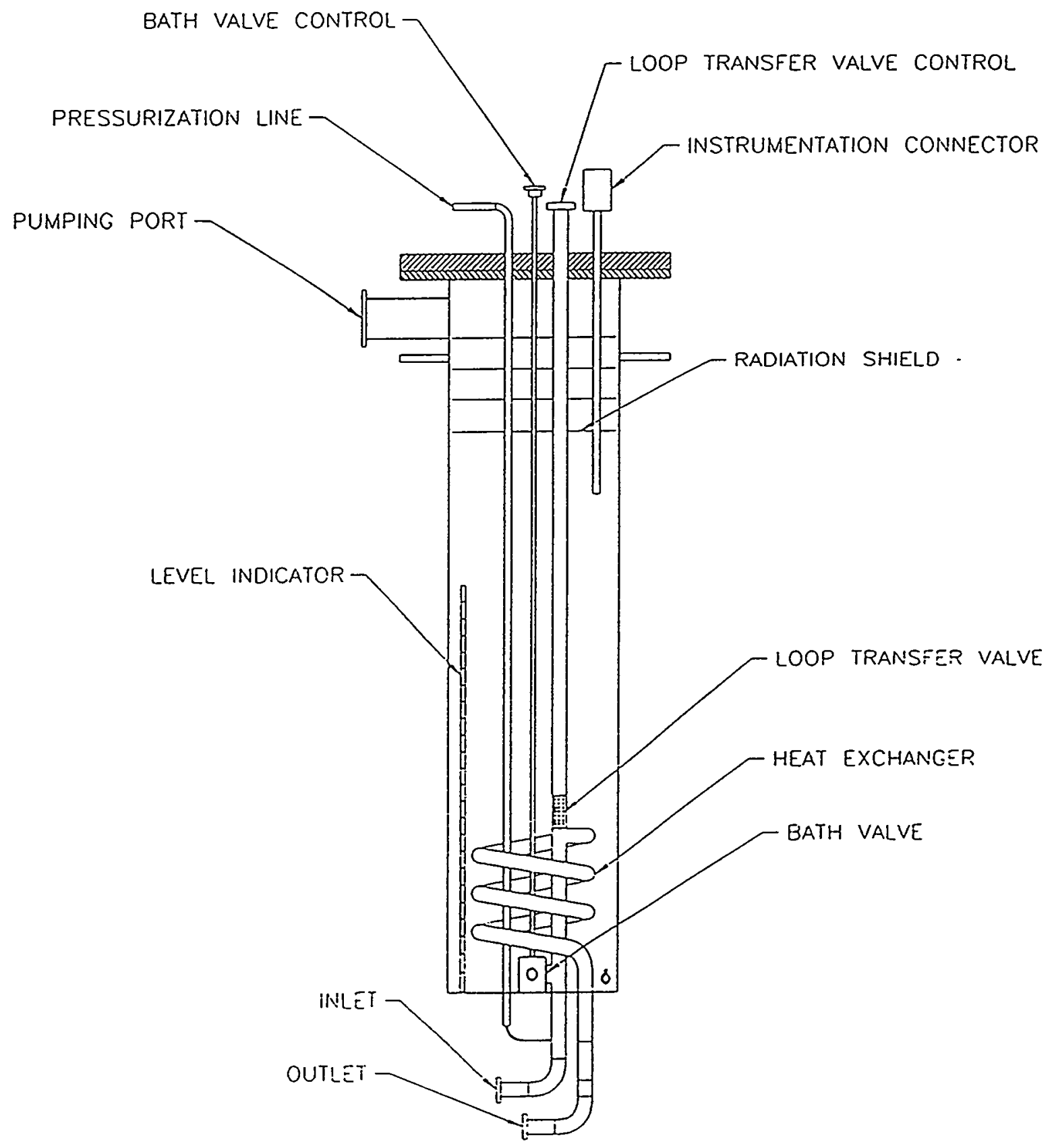

Figure 4.3. Schematic of the heat exchanger. 


\subsubsection{Experiment Loop}

Figure 4.4 shows the schematic view of the test loop for both the pressure drop measurement and the inductive heater transient pressure rise experiment. The loop consists of a prototype CICC, a venturi flow meter and a return loop. Each component is connected via indium o-ring seals for easy replacement of parts. The inductive heater is installed at the center of the conductor for the transient heat transfer and pressure rise experiments. A Pitot tube can be installed at the down stream end of a CEA conductor to measure low temperature helium velocity in a central channel of the conductor. Four stainless steel bellows are attached at the both ends of test section and return loop to accommodate the contraction of a system and provide some flexibility to connect the loop to the helium pump and the heat exchanger.

Three variable reluctance pressure transducers measure pressure drop across the CICC, the venturi flow meter and the Pitot tube. Each transducer is connected by a VCR metal gasket face seal fitting with an indium coated washer to insure vacuum tightness. Six differential pressure transducers (referenced of the vacuum space) measure the static pressure of the low temperature helium. The helium temperature in the loop was measured with germanium and/or Allen Bradley carbon resistance thermometers. Each thermometer was placed in an Oxygen Free High Conductivity (OFHC) copper tap. To mount the copper tap, a $3 \mathrm{~mm}$ diameter hole was drilled through the stainless steel jacket. To establish a good contact between the copper tap and the low temperature helium, all copper taps were silver soldered in place. Data acquisition wires were placed along the return loop and heat sunk with GE varnish at small copper tubes soldered in intervals along the loop. 


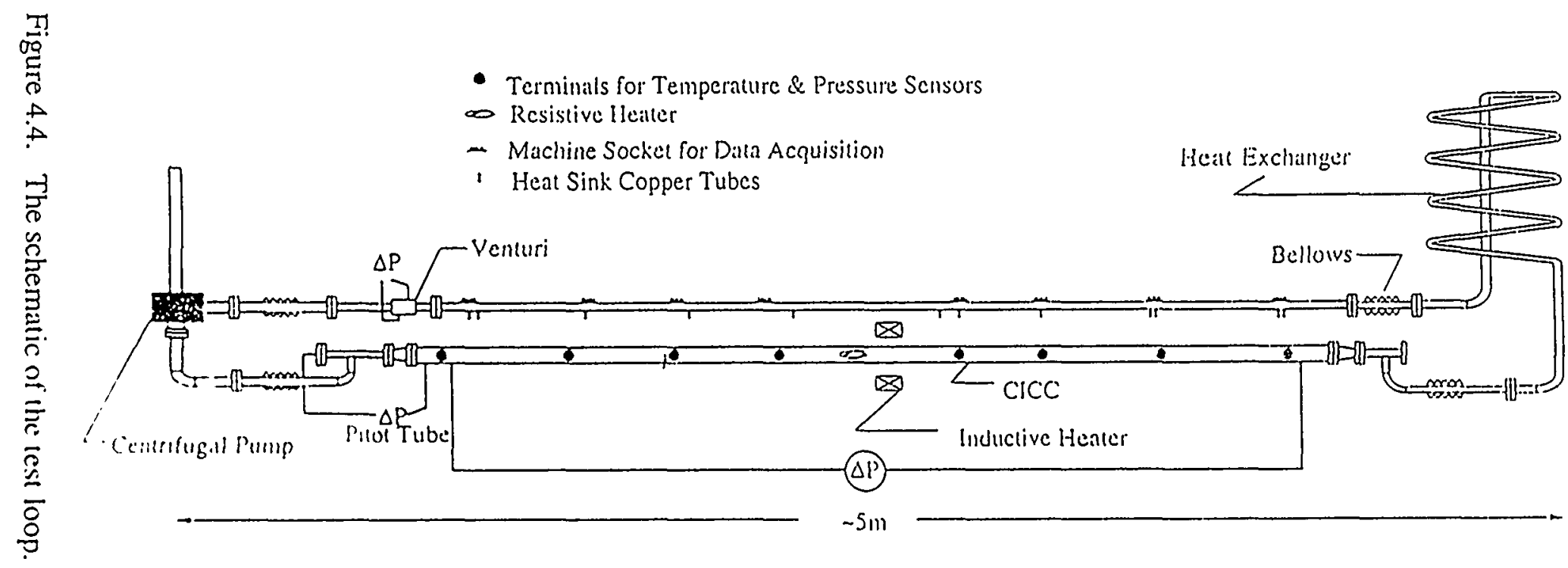




\subsubsection{Transient Heat Transfer Apparatus}

The transient heat transfer mechanisms within the prototype CEA conductor were studied with the experimental apparatus shown in Figure 4.5. A $0.32 \mathrm{~m}$ length of CICC was used with one end plugged with a copper block soft soldered to the stainless steel jacket. The other end was welded to stainless steel flange for installation in a vacuum can. This approach was chosen to avoid the complexity of using the LHFF.

The inductive heater was mounted with a micarta support at the bottom end of the conductor. Current leads for the inductive heater were cooled by a helium bath to minimize heat leaks from room temperature to the test section. The conductor can be connected or isolated from the helium bath through a BW series all welded bellows sealed Nupro valve. A pressurization line, $1 \mathrm{~m}$ long and $3 \mathrm{~mm}$ diameter copper tube, was coiled and soft soldered to the top flange. To regulate helium pressure within the CICC, the pressurization line was connected with a high purity helium gas cylinder through a pressure regulator. A liquid nitrogen cold trap was used to eliminate any impurities in the helium gas as it enters the test section. A Siemens KPY-45R pressure transducer located at room temperature, monitored pressure within the CICC during the experiment.

Both Allen Bradley carbon resistance thermometers and CERNOX thin film resistance thermometers were used for the experiment. The temperature of the helium in the central channel was measured with four Allen Bradley thermometers spaced along a $3 \mathrm{~mm}$ diameter stainless steel tube in the central channel. In order to get a fast response, the Allen Bradley thermometers were ground with sandpaper to expose the carbon. To measure the copper

strands temperatures, $3 \mathrm{~mm}$ diameter, $4 \mathrm{~mm}$ depth holes were drilled into the conductor and an OFHC copper rod was silver soldered in place. After that, a part of stainless steel jacket and OFHC copper was milled off to minimize eddy current generation. 


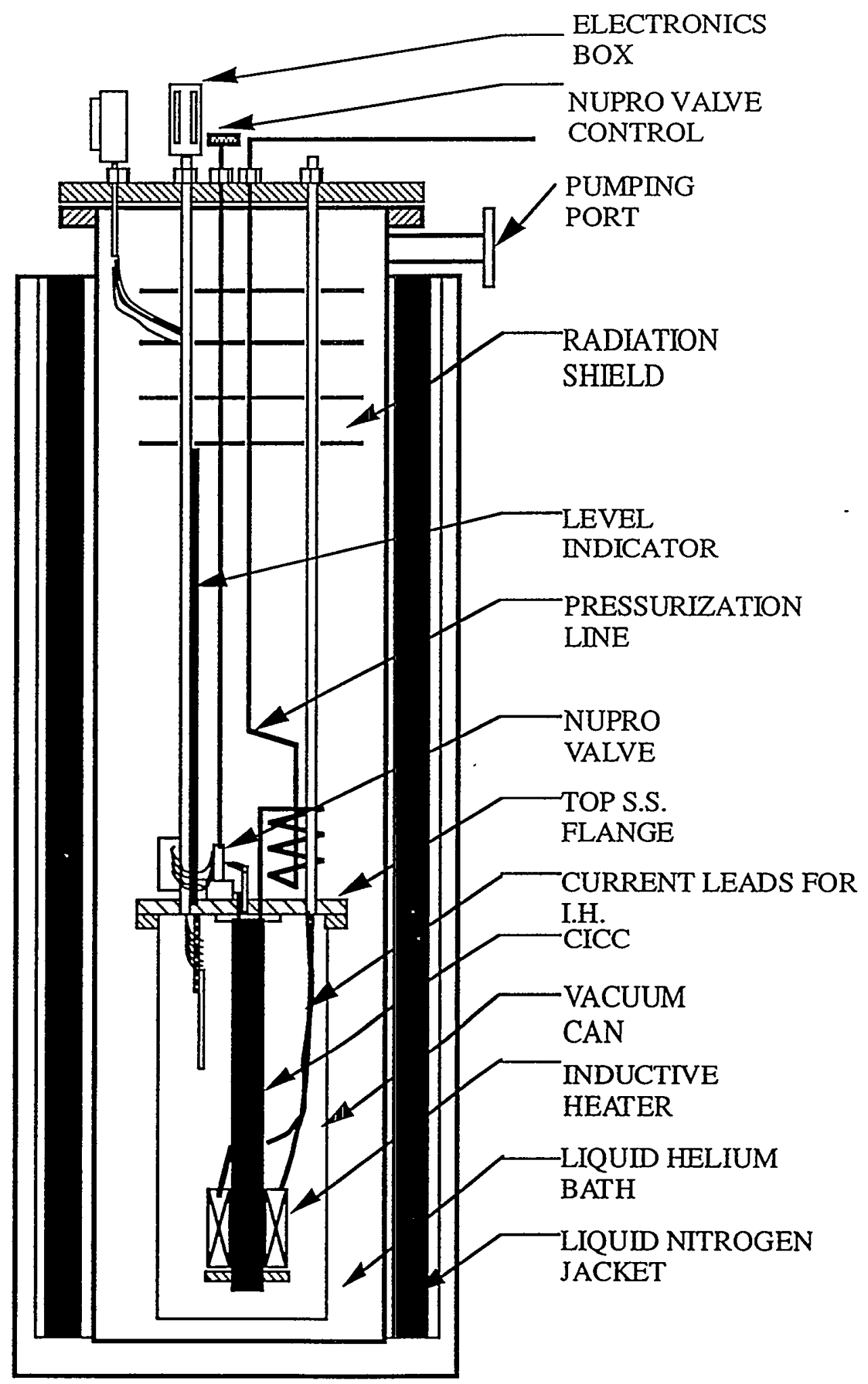

Figure 4.5. The schematic of transient heat transfer apparatus. 


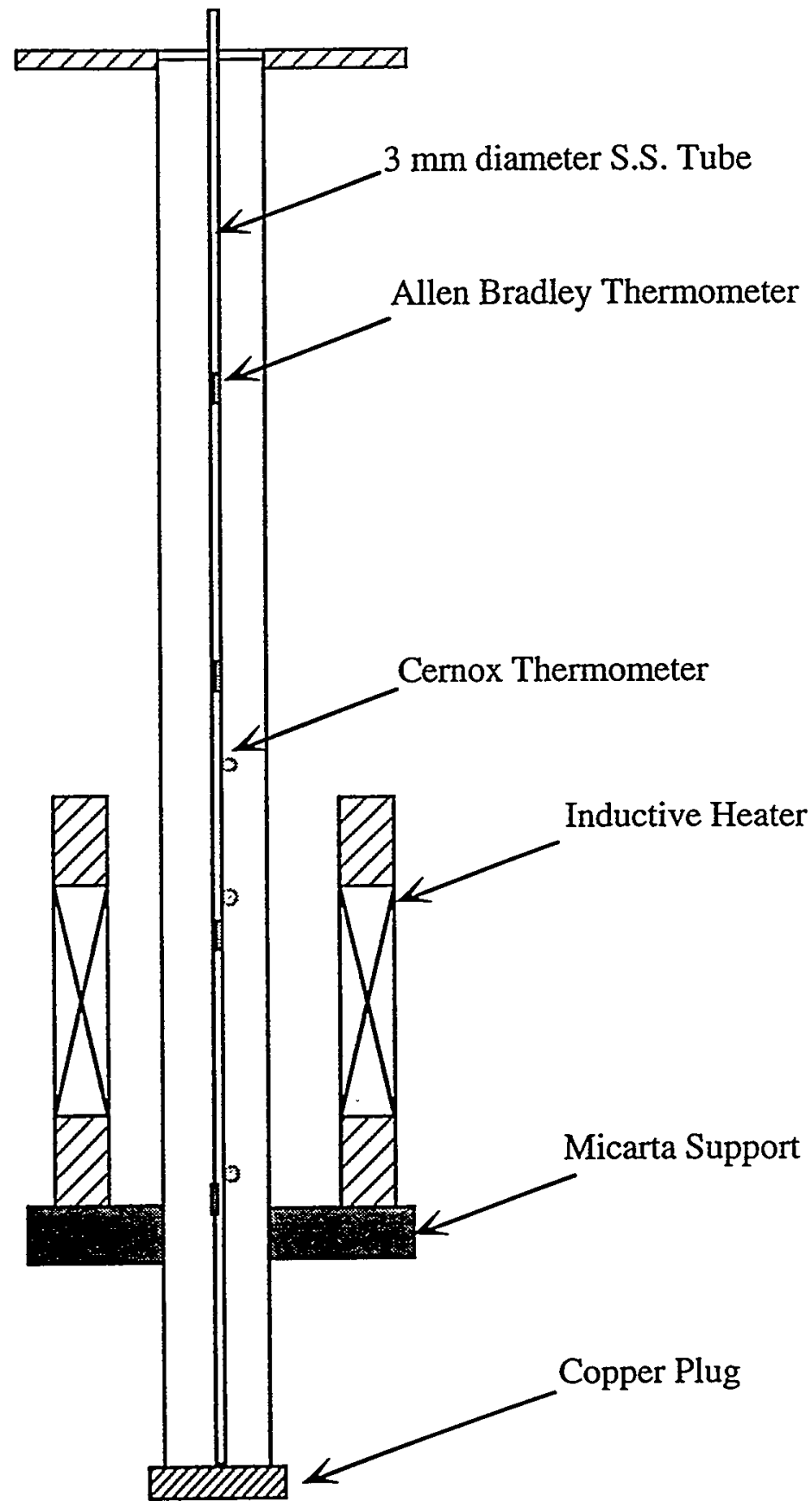

Figure 4.6. Transient heat transfer test section.

Figure 4.6 shows a test section for the transient heat transfer experiment with installation of the inductive heater and thermometers. Three CERNOX resistance thermometers were mounted on the surface of a copper post with GE varnish or indium solder. Two pairs of 
manganin wires measured the voltage and supplied constant current to thermometers. Those wires were anchored to the conductor jacket with GE varnish to insure good thermal contact and heat sinking. To minimize the self-heating effects of these thermometers, $0.5 \mu \mathrm{A}$ constant current was supplied with a battery type constant current power supply. The typical thermal response time of these thermometers is approximately $10 \mathrm{~ms}$ at $4.2 \mathrm{~K}$.

\subsubsection{Counterflow Heat Transport Experiment}

The apparatus for the counterflow experiment is almost the same as that used in the transient heat transfer experiment. The schematic of the test section is shown in Figure 4.7. The length of a conductor was approximately $0.32 \mathrm{~m}$ and bottom end was plugged with a copper block which was soft soldered to the conductor jacket. A $30 \mathrm{ohm}$ Minco film resistance heater was attached to a copper plug for various heat input to the He II in the short sample. Two $3 \mathrm{~mm}$ diameter holes were carefully drilled, without damaging the strands, to the both ends of the conduit so as to expose the inner space to measure the He II temperature. Two OFHC copper taps were silver soldered in the holes of the conduit. Each tap has a $1.5 \mathrm{~mm}$ diameter hole for mounting Allen Bradley carbon resistance thermometers.

The thermometers were ground with sandpaper to fit in the mounting hole since the contact between thermometer and the hole should be tight to insure a good thermal contact. Two pairs of twisted 36 gage manganin wires were soldered to the thermometer and wounded around the copper tap for the heat sink. Both thermometer and wires were covered with GE varnish to achieve a good thermal contact. Four wire measurement scheme is used for both thermometers and a heater. 


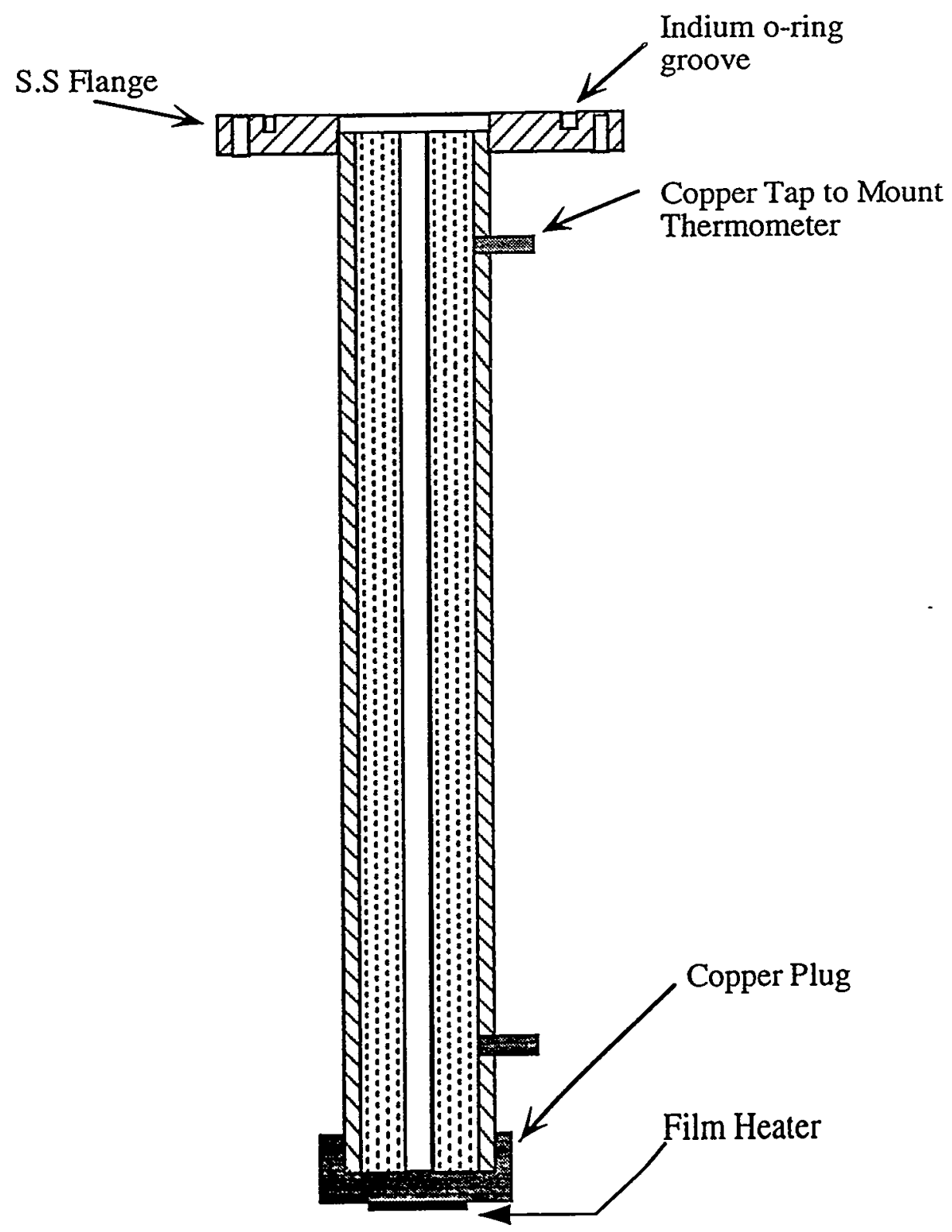

Figure 4.7. Test section of a counterflow experiment. 


\subsubsection{Data Acquisition System}

A data acquisition system for all experiments consists of a Macintosh Quadra 650 computer with a GW Instruments' MacADIOS II ${ }^{\mathrm{TM}}$ data acquisition board and a SuperScope $\mathrm{II}^{\mathrm{TM}}$ data acquisition program. A MacADIOS II has two Junction Field Effect Transistor (JFET) input multiplexers which can provide up to 8 differential analog input channels. In addition to this, an input multiplexer daughterboard ( Model \#GWI-MUX) can supply 16 differential analog input channels. Therefore, a total of 24 analog input channels can be used for data acquisition. Analog-to-digital conversion is performed by a 12-bit converter which is built in a MacADIOS II. The range of analog input is between $-10 \mathrm{~V}$ and $10 \mathrm{~V}$. The least significant bit value turns out to be $4.88 \mathrm{mV}$. MacADIOS also has two digital-to-analog output channels. It is possible for the data acquisition system to send some analog signals while working with analog-to-digital conversions. General signal path configuration for the data acquisition system is shown in Figure 4.8.

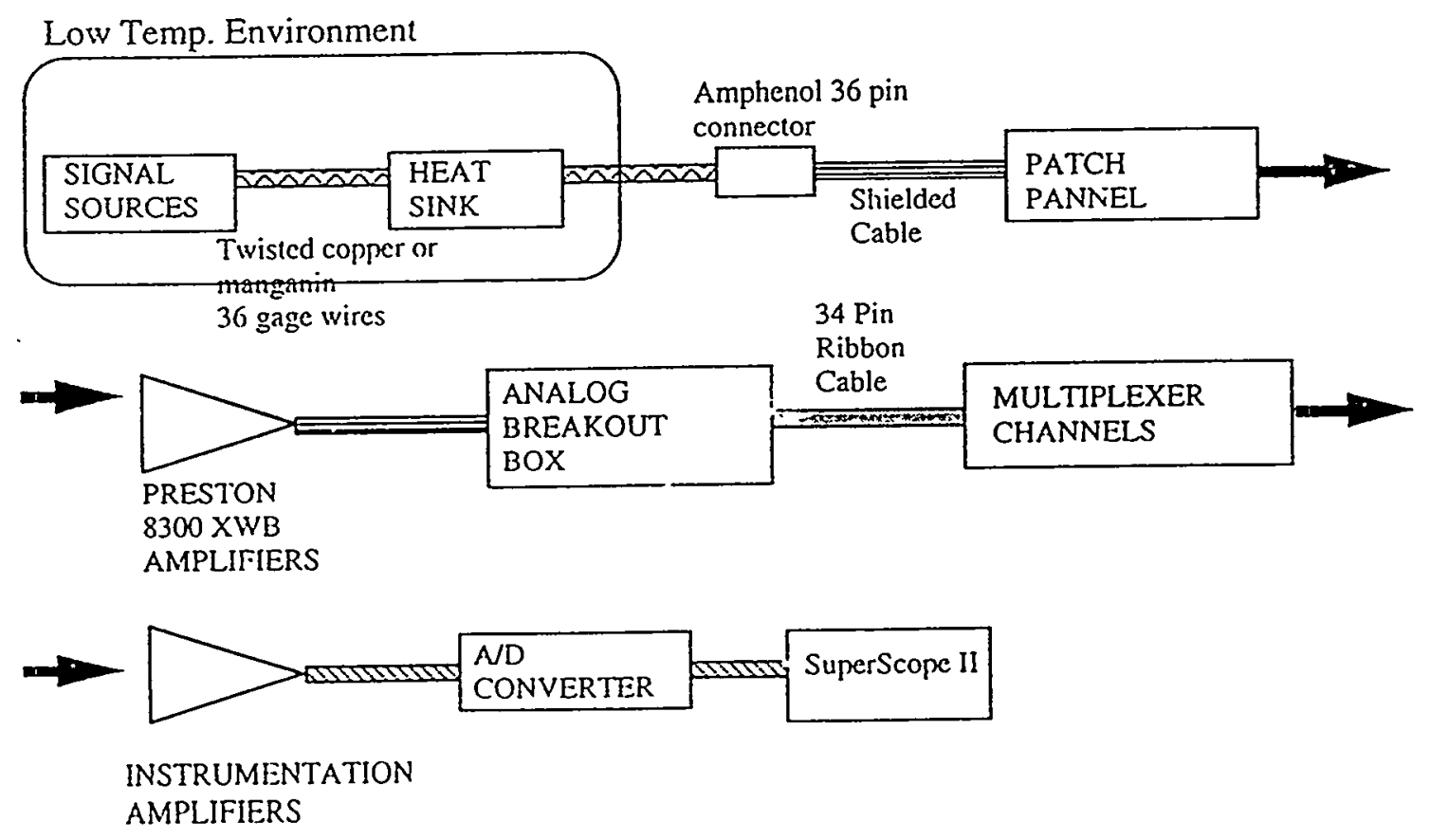

Figure 4.8 Data acquisition schematic. 
The wiring connection of MacADIOS with analog inputs and/or outputs is through a 34pin connector with a 34-pin ribbon cable which has two analog ground wires. The basic wiring scheme for the analog input is shown in Figure 4.9. $\mathrm{Rb}$ is a bias return resistance, typically $50 \mathrm{k} \Omega$, to prevent floating of the input signal.

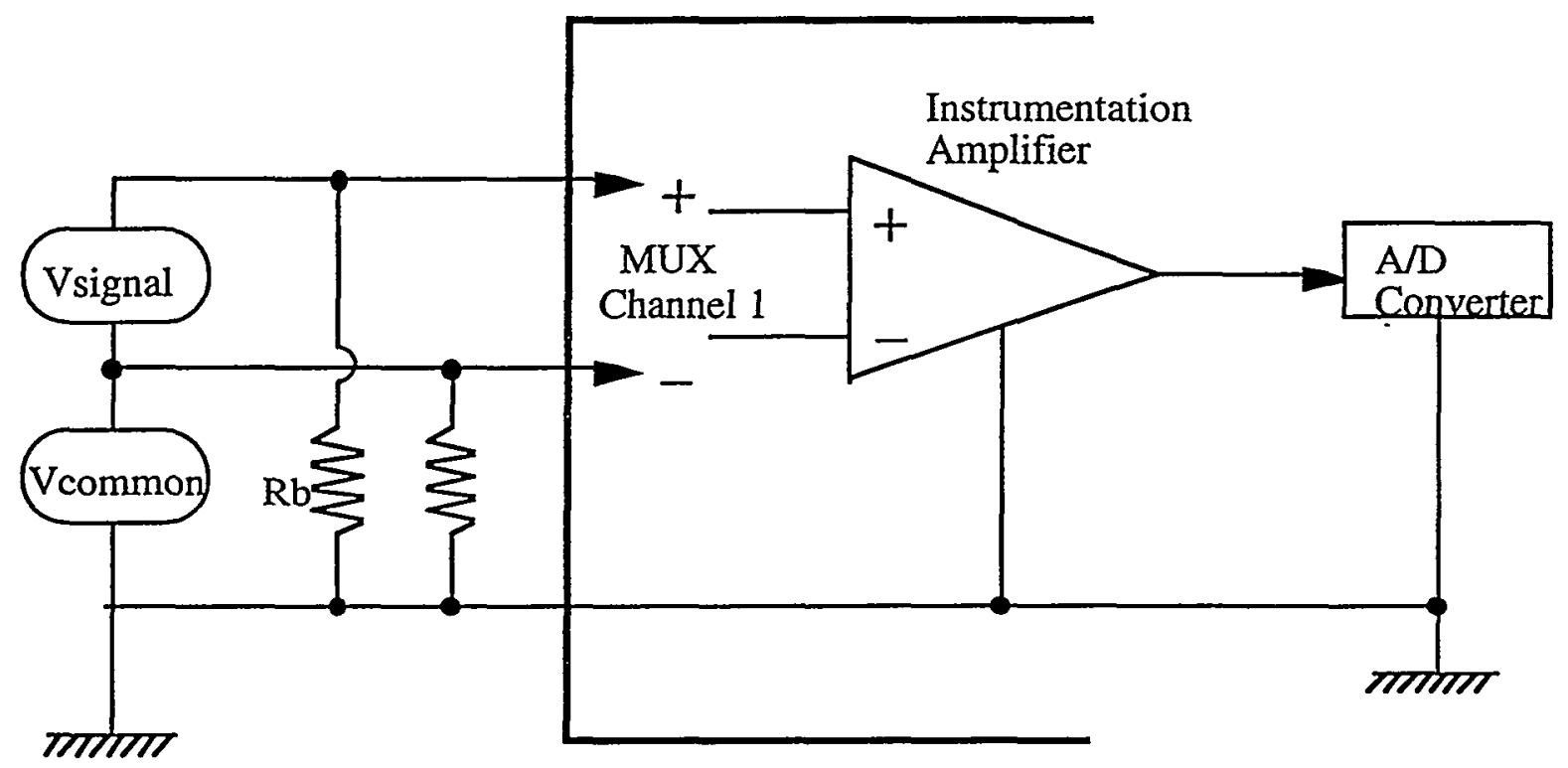

Figure 4.9. Typical wiring scheme for analog data input.

SuperScope II is a user friendly data acquisition software that can control MacADIOS II without an additional software program. SuperScope II consists of a number of software instruments which are a combination of word processor, data base and spreadsheet program for waveforms. These waveforms are mainly created by digitizing analog inputs as a function of time, such as readings from temperature sensors or pressure sensors. SuperScope II analyzes those wave forms and outputs analog signals via a digital-to-analog converter. 


\subsection{Temperature and Pressure Sensors}

Temperature sensors were chosen based upon the following criteria: repeatability with thermal cycles; sensitivity around a specific temperature range; and thermal time constant. Similarly, pressure transducers were selected for differential pressure measurements as well as absolute pressure measurements. The most important factor in selecting a pressure transducer is helium leak tightness at low temperature. Unfortunately, there are not many commercially available pressure transducers for this application.

\subsubsection{Germanium Resistance Thermometers}

Germanium resistance thermometers (GRT) are very popular for low temperature measurements, especially from $0.05 \mathrm{~K}$ to $30 \mathrm{~K}$. The sensor consists of a doped germanium chip on a strain free mounting and placed in a gold plated cylindrical cooper can. The space between a germanium chip and the can is filled with helium gas. Dimensions of the thermometer are $2.36 \mathrm{~mm}$ diameter and $6.1 \mathrm{~mm}$ long cylinder. Since its sensitivity increases rapidly with decreasing temperature, GRTs are useful below $5 \mathrm{~K}$. The remarkable characteristic of this sensor is its stability that has repeatability of $\pm 0.5 \mathrm{mK}$ at $4.2 \mathrm{~K}$. However, GRTs are expensive and must be carefully handled; particularly at a wiring connection to the sensor. Further, GRT response time is approximately $200 \mathrm{~ms}$ at $4.2 \mathrm{~K}$ so that it is not suitable to use in transient heat transfer experiments.

Equation (4.1) is an interpolation fit for a GRT. The fit to experimental data and obtain the coefficients: a0, a2,...

$$
\frac{1}{T}=a_{0}+a_{1} \ln (R)+a_{2}[\ln (R)]^{2}+a_{3}[\ln (R)]^{3}+\cdots
$$


where $a$ 's are numerical coefficients and $\mathrm{R}$ is sensor resistance. Figure 4.10 shows a typical calibration curve.

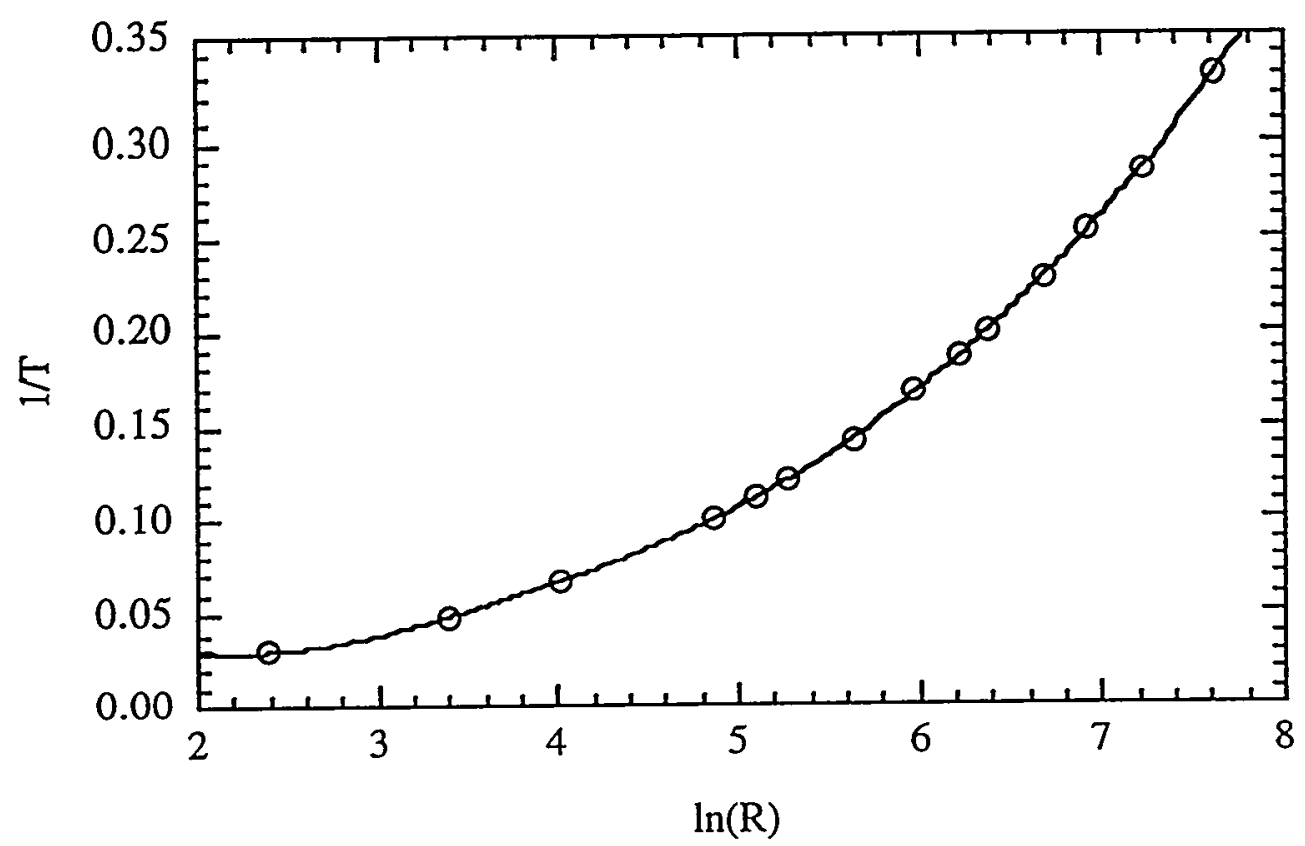

Figure 4.10 Calibration curve and data points for a germanium thermometer.

\subsubsection{Allen Bradley Carbon Resistance Thermometers}

Carbon resistance thermometers are also widely used below $20 \mathrm{~K}$, particularly in the He II temperature region. These thermometers are easy to handle and modify in size by simply grinding off some parts with sandpaper. The time response of this thermometer can be order of a few tens of milliseconds, depending on mounting arrangement. CRTs are generally inexpensive; however, calibration is necessary in situ since their calibration shifts with thermal cycling. Power dissipation is approximately $5 \mathrm{nW}$ at $1.8 \mathrm{~K}$ and $0.8 \mathrm{nW}$ at $4.2 \mathrm{~K}$ with I $\mu \mathrm{A}$ 
nominal current. Calibration data can be fit with Equation (4.1) for temperatures below $T_{\lambda}$; however, the equation used to fit between $2 \mathrm{~K}$ and $20 \mathrm{~K}$ is given by,

$$
\left[\frac{\log R}{T}\right]^{1 / 2}=A+B \log R
$$

where $\mathrm{A}$ and $\mathrm{B}$ are numerical coefficients. Figure 4.11 shows data points fit with Equation (4.2).

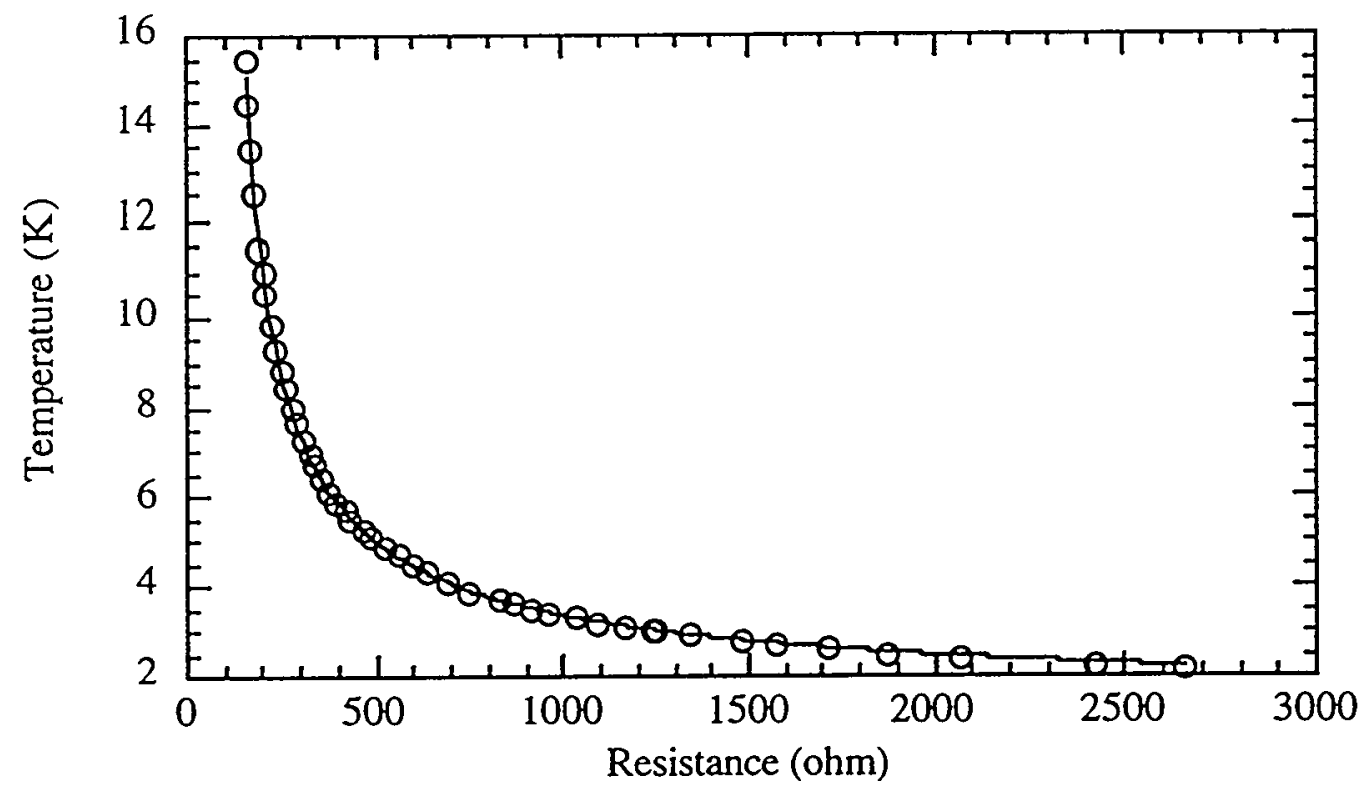

Figure 4.11. Typical calibration curve for an Allen Bradley thermometer. 


\subsubsection{CERNOX Resistance Thermometers}

Lake Shore Cryotronics CERNOX ${ }^{\mathrm{TM}}$ thin film resistance thermometers are used to study transient heat transfer of a CEA conductor because of its fairly quick time response $\sim 10 \mathrm{~ms}$ at $4.2 \mathrm{~K}$ and high sensitivity at low temperature region $\mathrm{dR} / \mathrm{dT} \sim 10^{5} \mathrm{ohms} / \mathrm{K}$ at $4.2 \mathrm{~K}$. This sensor consists of a ceramic oxynitride chip with gold pads mounted on a sapphire substrate. The dimension of a sensor is shown in Figure 4.12. CERNOX sensors have excellent stability against thermal cycling.

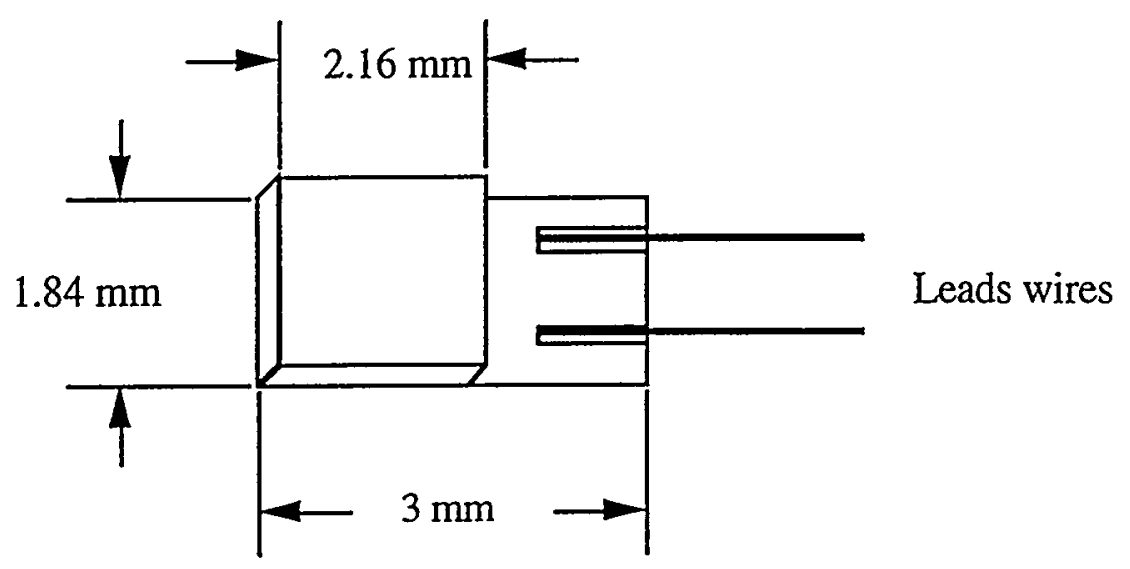

Thickness is approximately $0.98 \mathrm{~mm}$

Sensor mass is $0.03 \mathrm{~g}$

Figure 4.12. Dimension of cernox thin film thermometer.

Figure 13 shows typical calibration curve for the CERNOX thermometer with Equation (4.1). 


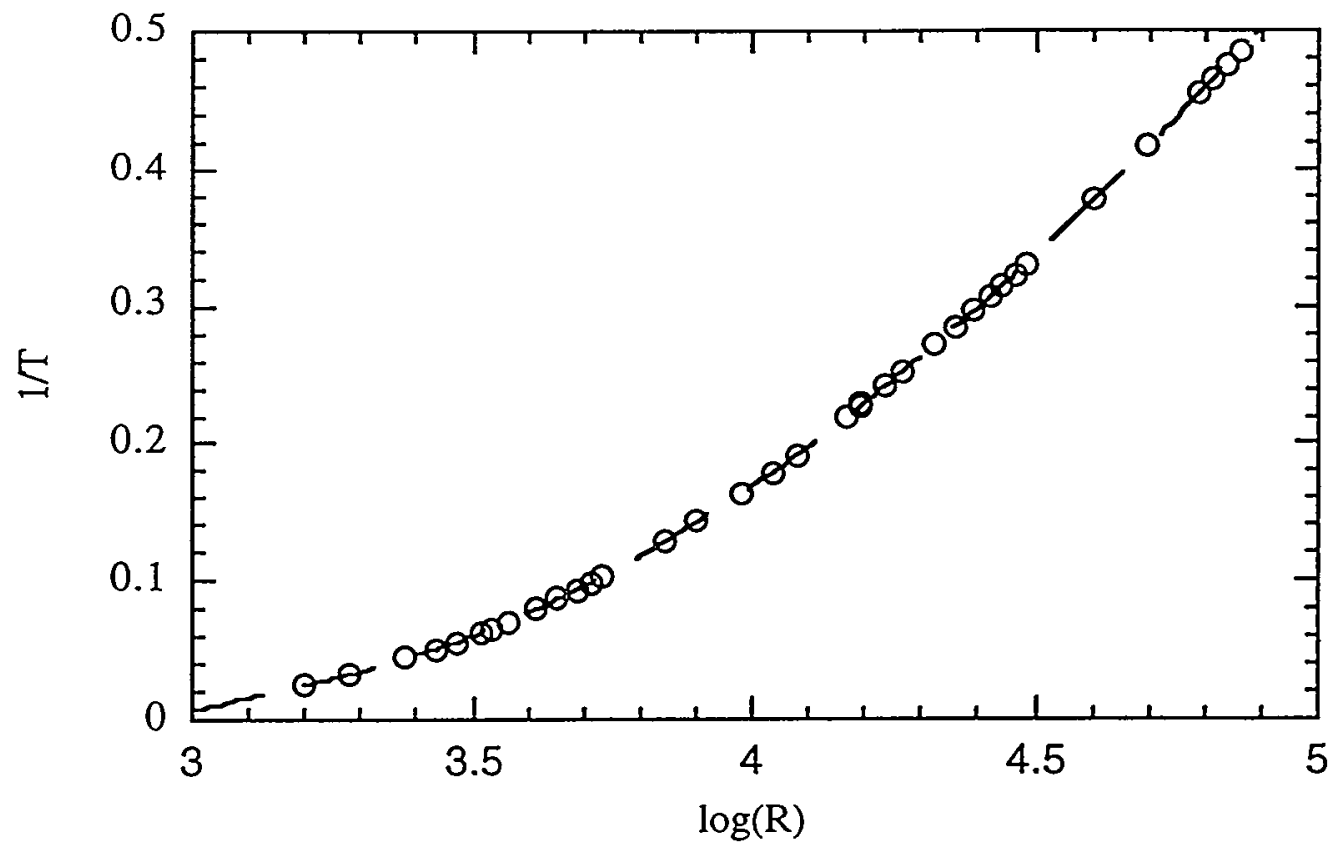

Figure 4.13. Calibration curve for Cernox thermometer.

\subsubsection{Calibration of Temperature Sensors}

Two types of calibration methods were used; one involved calibration against a commercially calibrated thermometer, the other calibration against the helium vapor pressure. The former method was used to calibrate from $3 \mathrm{~K}$ to $35 \mathrm{~K}$, while the latter method was used in the $\mathrm{He}$ II temperature regime. In a first case, a temperature calibration rig was used to simultaneously calibrate up to five thermometers. Thermometers were carefully mounted in the OFHC copper plate with either Crycon ${ }^{\mathrm{TM}}$ grease or GE varnish to insure good thermal contact. The copper plate was then installed on the calibration section, located in the vacuum can. A commercially calibrated carbon glass resistance temperature sensor was installed in the section as a standard thermometer. The whole system was immersed in a liquid helium bath. 
A Lake Shore DRC-91CA temperature controller was used to maintain various constant temperatures of the copper plate between $3 \mathrm{~K}$ and $35 \mathrm{~K}$. In most cases, depending on temperature, it took approximately five to ten minutes to reach a steady state. More than 20 data points were taken to generate the calibration curve for each thermometer.

For He II temperature region, thermometers were either directly immersed in a He II bath or mounted on an OFHC copper block which was cooled by He II. The He II bath pressure was varied from 10 Torr to 37 Torr. Approximately 13 data points were taken between above pressure region. A MKS Type 102A 100 Torr pressure transducer with reading error of 0.5 $\%$ was used to measure the helium bath pressure. The He II temperature was obtained from a fit to the vapor pressure,

$$
T=\sum_{i=1}^{8} b_{i} X_{i}
$$

where

$$
X_{i}=\frac{\ln (P)-A}{B}
$$

and $b_{i}, A$ and $B$ are coefficients based upon Rusby's results. 45

Figure 4.14 shows the thermometer sensitivity comparison for the three sensors. The CERNOX thermometer has about an order of magnitude higher sensitivity than the Allen Bradley and GRT. This makes the CERNOX sensors suitable for use above $10 \mathrm{~K}$. 


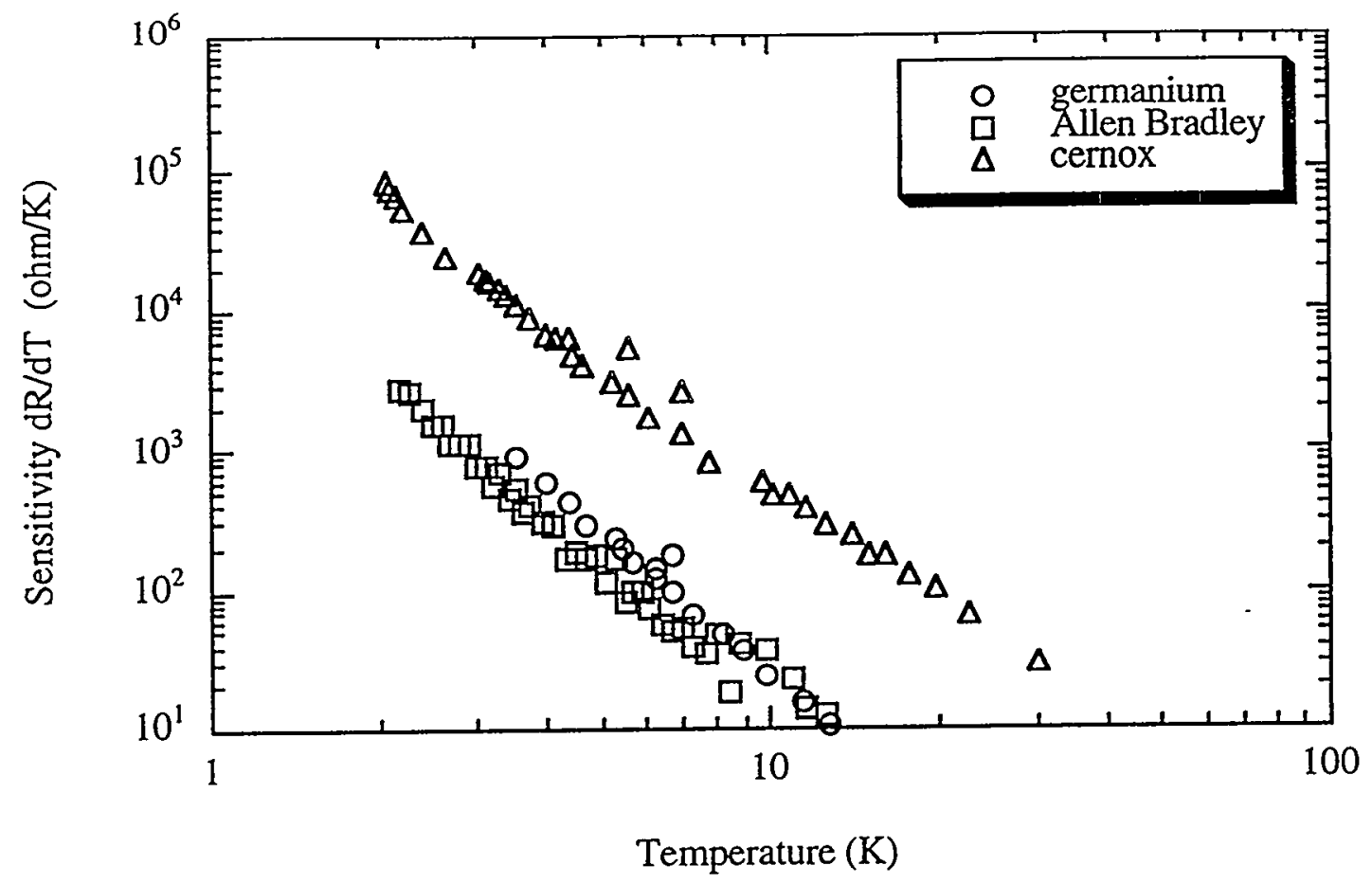

Figure 4.14. Sensitivity for each thermometers at different temperature.

\subsubsection{Variable Reluctance Pressure Transducer}

Validyne DP-10 series all welded differential pressure transducers were used to measure pressure drop across the CICC, venturi flow meter and Pitot tube. Although these pressure transducers are not designed for the liquid helium temperature environment, they produce reliable pressure drop readings and are helium leak tight at low temperature. The principle of operation mechanism of the pressure transducer is as follows. A magnetic stainless steel diaphragm symmetrically separates a pressure cavity with each space connected to a pressure port for differential pressure measurements. The diaphragm deflects as a result of pressure 
difference between two ports, which induces a magnetic reluctance change. These changes are monitored with two pick up coils, which are mounted just outside the cavity.

A 5V rms. energizes the two coils which are part of a bridge circuit. An AC output voltage from a coil is demodulated to a DC output voltage which is proportional to the pressure difference between two ports. These pressure transducers can withstand the $200 \%$ of its full scale pressure difference. However, since these pressure transducers are very expensive, a maximum allowable pressure drop has to be determined by the lowest full scale pressure transducer if several pressure transducers are used in the same test loop.

The calibration of transducers at low temperature is necessary. Figure 4.11 shows a pressure transducer calibration schematic.

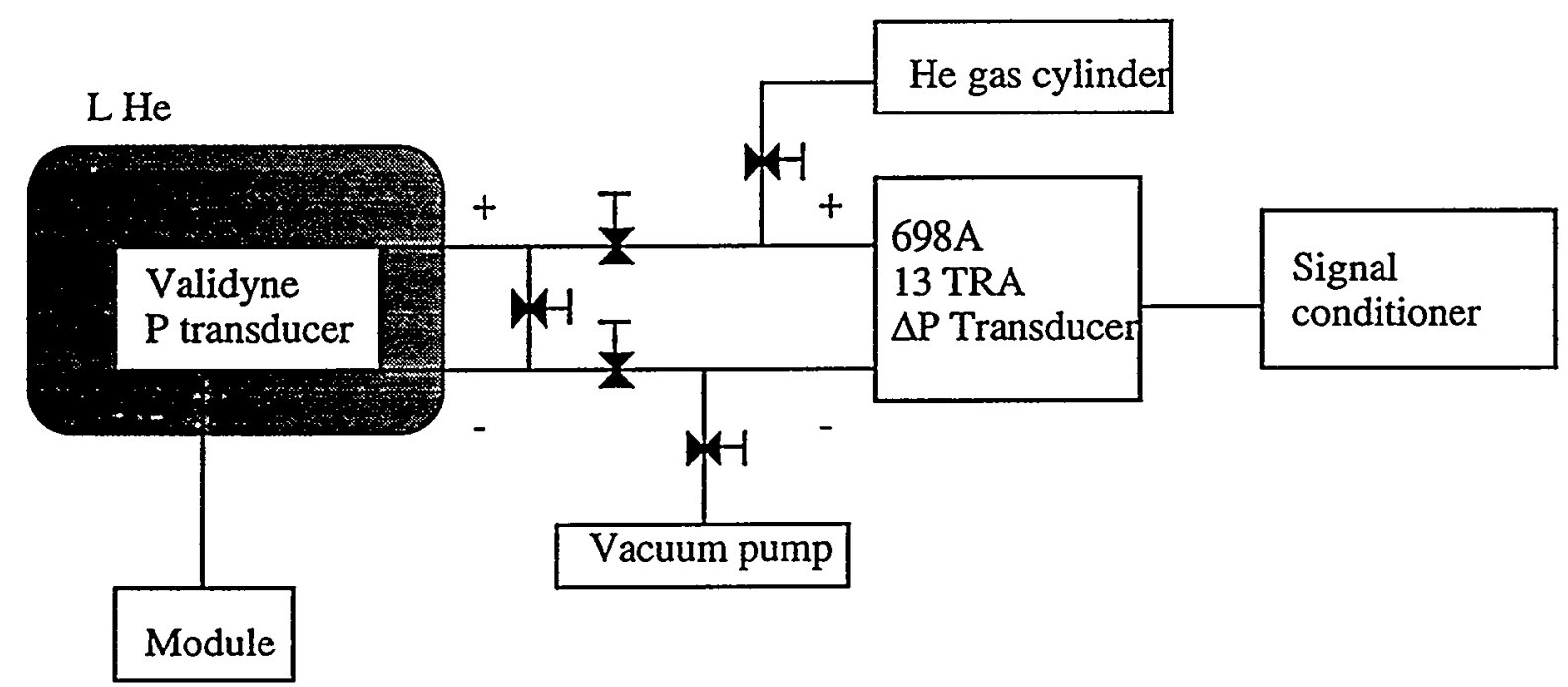

Figure 4.11. Diagram of a pressure sensor calibration. 
Two differential pressure transducers can be calibrated at the same time. To prevent over pressurizing transducers, the control valve is left open until the calibration. After liquid helium is transferred to the bath, the calibration can be performed by pressurizing a test port with helium gas. The test port pressure is controlled with a needle valve. A Baratron Model $698 \mathrm{~A}$ 13TRA 1000 Torr differential transducer with MKS Type 270 Signal Conditioner was used as a standard pressure which has $0.13 \mathrm{~Pa}$ resolution. The output from DP-10 differential pressure transducers are almost linear as a function of pressure.

\subsubsection{Cryogenic Pressure Transducer}

Pressure Systems Inc. (PSI) cryogenic series 4000 pressure transducers were used to measure static pressure of low temperature helium. These pressure transducers are specifically designed for cryogenic experiments. Their operating range is from $1.5 \mathrm{~K}$ to $400 \mathrm{~K}$. An all welded stainless steel housing structure provides the helium leak tight component. The transducer has a piezoresistive silicon pressure sensor inside the stainless steel housing. The accuracy of this sensor is approximately $0.5 \%$. Calibration of a pressure transducer is necessary at different temperatures since output from the sensor depends on operating temperatures. A fourth order polynomial equation is used to fit the calibration results,

$$
P(V)=a_{0}+a_{1} V+a_{2} V^{2}+a_{3} V^{3}+a_{4} V^{4}
$$

A $1 \mathrm{~mA}$ constant current power supply energizes pressure transducers with nominal power dissipation of $6 \mathrm{~mW}$. 


\subsubsection{Siemens KPY Pressure Transducer}

A Siemens KPY-45R pressure transducer was also used to measure static pressure of supercritical helium at low and room temperature. Although the signal output from KPY transducer is a linear function of pressure, the calibration slope changes with temperature. Therefore, it is necessary to calibrate them in situ. The calibration of sensors were conducted, using both Barocel Pressure Sensor with 1000 Torr range and a pressure dial gage on the regulator.

\subsection{Experimental Procedure}

\subsubsection{Counterflow Heat Transport Experiment}

After the experimental apparatus for the counterflow experiment was installed in the cryostat, the whole system was purged with high purity helium gas to prepare for precooling. Liquid nitrogen was then transferred to the liquid nitrogen jacket of a cryostat. At this point, the helium bath was filled and maintained with exchange helium gas at approximately $1 \mathrm{~atm}$. Liquid helium transfer was accomplished about 24 hours after a pre-cooling with exchange

gas. It took about $40 \mathrm{~min}$. to fill liquid helium in the bath. A $0.61 \mathrm{~m}$ long American Magnetics liquid helium level indicator monitored the helium level.

The helium bath was slowly pumped to the He II region ( $\mathrm{P}<37$ Torr ) in about an hour. The bath pressure was then automatically controlled with an MKS type 252A exhaust valve controller. After the bath pressure reached steady state or a specified He II temperature, a Minco film resistance heater was energized with a Kepco model ATE 100-1M power supply in constant voltage mode. Data were taken, once thermometer reading reached a steady state 
condition. Both thermometer outputs were monitored with a Linseis L7005, type 7045 chart recorder. A steady state temperature distribution usually took a few minutes. Measurements stopped when the heater power exceeded the peak heat flux and the thermometer output started to oscillate. Three different $\mathrm{He}$ II bath temperatures $(1.8 \mathrm{~K}, 1.9 \mathrm{~K}$ and $2.05 \mathrm{~K})$ were investigated in the experiment.

\subsubsection{Pressure Drop Measurements}

After installation of the CICC test loop in the LHFF, the entire loop was purged five or six times with high purity helium gas. At the same time, the inner and the outer isolation vacuum spaces were pumped. Liquid nitrogen was transferred to liquid nitrogen jackets of two vertical cryostats and the liquid nitrogen reservoir of horizontal cryostat after about a day of pumping. A refrigeration system for a $10 \mathrm{~K}$ radiation shield started after two days of precooling with liquid nitrogen.

The test loop was cooled to about $100 \mathrm{~K}$ with helium exchange gas in the inner space of the LHFF. Liquid helium was then transferred from a control valve through the test loop and to the helium bath. After the bath was filled with liquid helium, the heat exchanger bath was pumped down to He II ( $\mathrm{P}<37$ Torr $)$ to fill the loop with liquid. The control valve of the heat exchanger was left open during the above operations. After the temperature of the test loop reached about $1.9 \mathrm{~K}$, the control valve was closed to isolate the test loop from the helium bath.

The test loop pressure was adjusted to the desired value through a pressurization line with a pressure regulator attached to a high pressure helium gas cylinder. To eliminate any impurities in helium gas, liquid nitrogen was used as a cold trap. The centrifugal helium pump was run at a low frequency $(-25 \mathrm{~Hz})$ to keep constant temperature in the loop. It took about $45 \mathrm{~min}$. to pressurize the loop to approximately $0.3 \mathrm{MPa}$. 
As soon as supercritical helium in the loop reached a steady state condition, pressure drop measurements were carried out as a function of various pumping speeds. The experiments were conducted by increasing and decreasing pumping speed to study hysterisis. A differential pressure transducer on the CICC was monitored with a chart recorder during the measurements, which was used to determine when the flow reached a steady state as changing the pumping speed.

\subsubsection{Inductive Heater Transient Pressure Rise Experiment}

After steady state condition for either $\mathrm{He}$ II or supercritical helium was established in the test loop, the capacitor bank was charged to a certain value. Discharging the capacitor bank was initiated with the data acquisition program by sending a synthesized two analog square pulse that has $16 \mathrm{~ms}$ period with $\pm 5 \mathrm{~V}$ peak-to peak. Data were taken for $8 \mathrm{~ms}$ before sending the pulse. The sampling rate of data varied from 1 to $8 \mathrm{~ms}$ per points for each of 16 channels. Experiments were conducted as function of different mass flow rate, charged energy to the capacitor bank. It took about 5 to $10 \mathrm{~min}$. for the system to cool down to the original temperature so that each shot was taken at about $15 \mathrm{~min}$. interval.

\subsubsection{Transient Heat Transfer Experiment}

The precooling procedure was the same as for the counterflow heat transport experiment. For the supercritical helium experiment, the Nupro valve was closed and pressure within a short conductor sample adjusted to approximately $0.3 \mathrm{MPa}$ through a pressure regulator attached to a helium gas cylinder. The capacitor bank was energized to a desired value. Discharging of the capacitor was initiated by a Macintosh computer with Superscope II data acquisition software. 
The data acquisition period was easily adjusted to give different energy input to the conductor since higher energy input to the conductor took a longer time to reach steady state condition. The Nupro valve was opened to cool down the conductor after each shot. Different temperature sensors were monitored to verify the helium and the copper strand temperature returned to $4.2 \mathrm{~K}$. The same procedures were repeated once the conductor temperature reached $4.2 \mathrm{~K}$ for different energy input.

For the He II experiment, the liquid helium bath was pumped to approximately 12.4 Torr, corresponding to $\mathrm{T}_{\mathrm{b}}=1.8 \mathrm{~K}$. This experiment was carried out under saturated vapor pressure. To isolate the test section to the He II bath, the Nupro valve was closed just before discharging the capacitor. The Nupro valve was opened to cool down the conductor for the next shot. 


\section{Chapter 5. Results and Analysis}

The present chapter reports the experimental results and analysis for both NET and CEA Cable-in-Conduit Conductors. First, three helium flow area characterization methods are discussed: the computer image analysis; the counterflow heat transport results and the geometrical component calculation. Second, the results of pressure drop measurements are presented in terms of classical friction factor and Reynolds number relationship. Several correlations are used to fit the data. Further, the flow modeling in the CEA conductor is discussed in detail. The model appears to provide practical hydraulic characterization of the hybrid CICC. Third, calibration results of the inductive heater are described with its method and analysis, followed by the thermally induced flow and transient pressure rise results for the CEA conductor. The parameters which affect the thermally induced flow are discussed. Thermal hydraulic characteristics of the CEA conductor with reduced central channel area is also presented. This result indicates the central channel of the conductor dominates the overall hydraulic characteristics. Finally, the transient heat transfer measurements with static supercritical helium and $\mathrm{He}$ II are presented.

\subsection{Characterization of the NET and CEA conductors}

Two prototype Cable-in-Conduit Conductors were used for this work. The NET (Next European Torus) conductor is a conventional CICC which consists of 588 strands uniformly distributed in the rectangular stainless steel conduit. The CEA (Commissariat a l'Energie Atomique) conductor is a hybrid CICC consisting of a central cooling channel that is surrounded by six bundles. Each bundle is helically wrapped with a thin stainless steel strip 
and contains 144 copper strands. There is no tube or spring like coil to separate the central channel and bundles.

The present section provides three methods to characterize the CICC cross sections: computer image analysis; the nominal geometry component; and a counterflow analysis The first method uses a computer to analyze a photographic cross section. The analyses provide the flow areas and cooled perimeters. The nominal geometry component method is simply obtained by calculating those parameters based upon the nominal diameter of a strand. The third method utilizes the heat transport mechanism of $\mathrm{He}$ II to estimate the effective heat transfer area. It is crucial to determine the helium flow area within the conductor since it significantly affects the friction factor calculation. The difficulty in characterizing the cross section of the CICC is primarily due to its multistage strands construction and the number of strands occupied in the conductor. In general, the final stage of the strands consists of four or five sub-stages.

\subsubsection{Image and Geometrical Component Analyses}

The short CIC conductor sample was filled with epoxy to fix the strands and cut to expose the cross section. The cross section was then polished and a picture was taken. A Megavision image analysis computer transferred the photograph into a 1024 by 1024 pixel image. The image is then converted to a binary mode: white flow area and black strands area. The computer counts these pixels to determine the flow cross sectional area, strands area and cooled perimeter. Figure 5.1 shows the captured image for both NET and CEA conductors. 


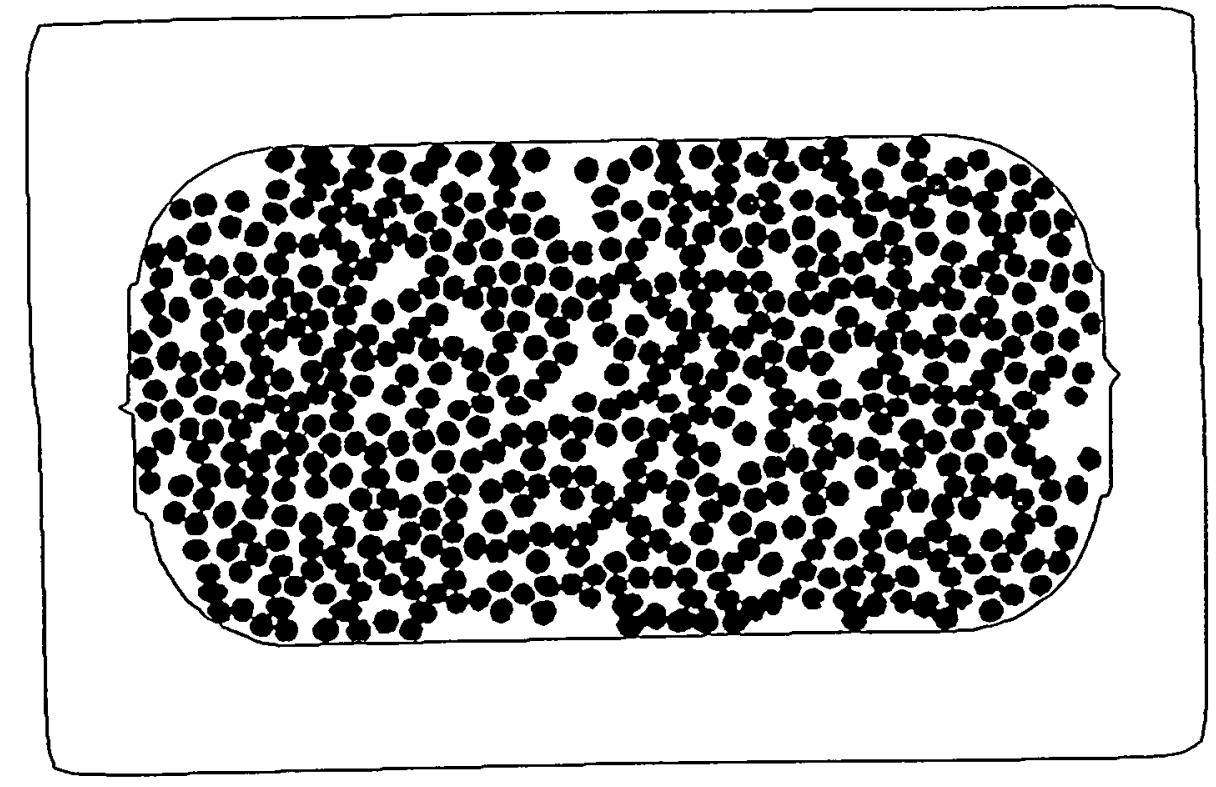

Figure 5.1. Binary image of NET conductor.

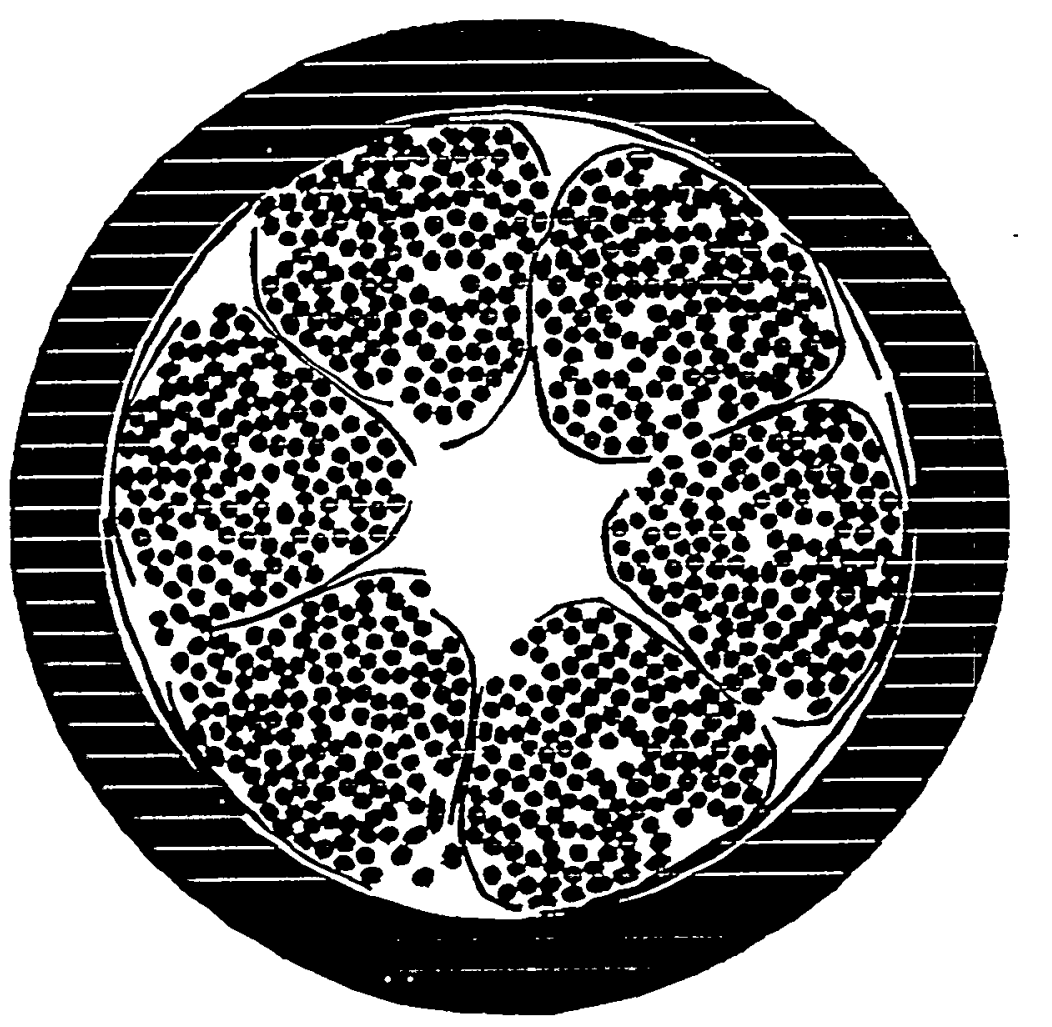

Figure 5.2. Binary image of CEA conductor 
The image analysis results for both NET and CEA conductors are shown in Table 5.1.

\begin{tabular}{ccc}
\hline & NET & CEA \\
\hline Strand diameter & $0.94 \mathrm{~mm}$ & $0.70 \mathrm{~mm}$ \\
Number of strands & 588 & 864 \\
Strands area & $410 \mathrm{~mm}^{2}$ & $336 \mathrm{~mm}^{2}$ \\
Flow area & $364 \mathrm{~mm}^{2}$ & $343 \mathrm{~mm}^{2}$ \\
Sheath area & N/A & $51.8 \mathrm{~mm}^{2}$ \\
Void fraction & $47 \%$ & $47 \%$ \\
Cooled perimeter & $1.68 \mathrm{~m}$ & $2.29 \mathrm{~m}^{2}$ \\
Cable pattern & N/A & $3 \times 3 \times 4 \times 4 \times 6$ \\
Hydraulic diameter & $0.87 \mathrm{~mm}$ & $0.60 \mathrm{~mm}$ \\
\hline
\end{tabular}

Table 5.1 The image analysis results with some other parameters.

The nominal geometric component analysis is calculating the conductor parameters based upon a nominal strand diameter. The calculation results are summarized in Table 5.2.

\begin{tabular}{ccc}
\hline & NET & CEA \\
\hline Strand diameter_n & $0.96 \mathrm{~mm}$ & $0.73 \mathrm{~mm}$ \\
Strands area_n & $426 \mathrm{~mm}^{2}$ & $362 \mathrm{~mm}^{2}$ \\
Flow area_n & $348 \mathrm{~mm}^{2}$ & $317 \mathrm{~mm}^{2}$ \\
Void fraction_n & $45 \%$ & $43 \%$ \\
Cooled perimeter_n & $1.89 \mathrm{~m}$ & $2.26 \mathrm{~m}$ \\
Hydraulic diameter_n & $0.73 \mathrm{~mm}$ & $0.56 \mathrm{~mm}$ \\
\hline
\end{tabular}

Table 5.2 Nominal geometric component calculation results. 


\subsubsection{Counterflow Analysis}

Internal convection theory of $\mathrm{He}$ II can be used to estimate the heat transfer area within a CICC. It is possible to estimate the area by solving the integration of the one dimensional turbulent heat transport equation:

$$
I\left(T_{b}\right)=q^{3}=\frac{1}{L} \int_{T_{b}}^{T_{\max }} \frac{1}{f(T)} d T
$$

where $f(T)$ is the same as Equation (2.11), $q$ is the heat flux, $L$ is the distance between thermometers and $T_{b}$ is the bath temperature. The evaluation of Equation (5.1) is equivalent to solving a first order differential equation with the initial condition $I\left(T_{b}\right)=0$. The fourthorder Runge-Kutta method was used to evaluate the equation.

Three different He II bath temperatures were used to estimate the area. Figures 5.3 (a), (b) and (c) show the experimental results of the temperature difference established along the conductor versus applied heat flux with the theoretical calculation. The fitting parameter between the experiment and the calculation results is the heat transfer area $A_{\text {fow }}$. 


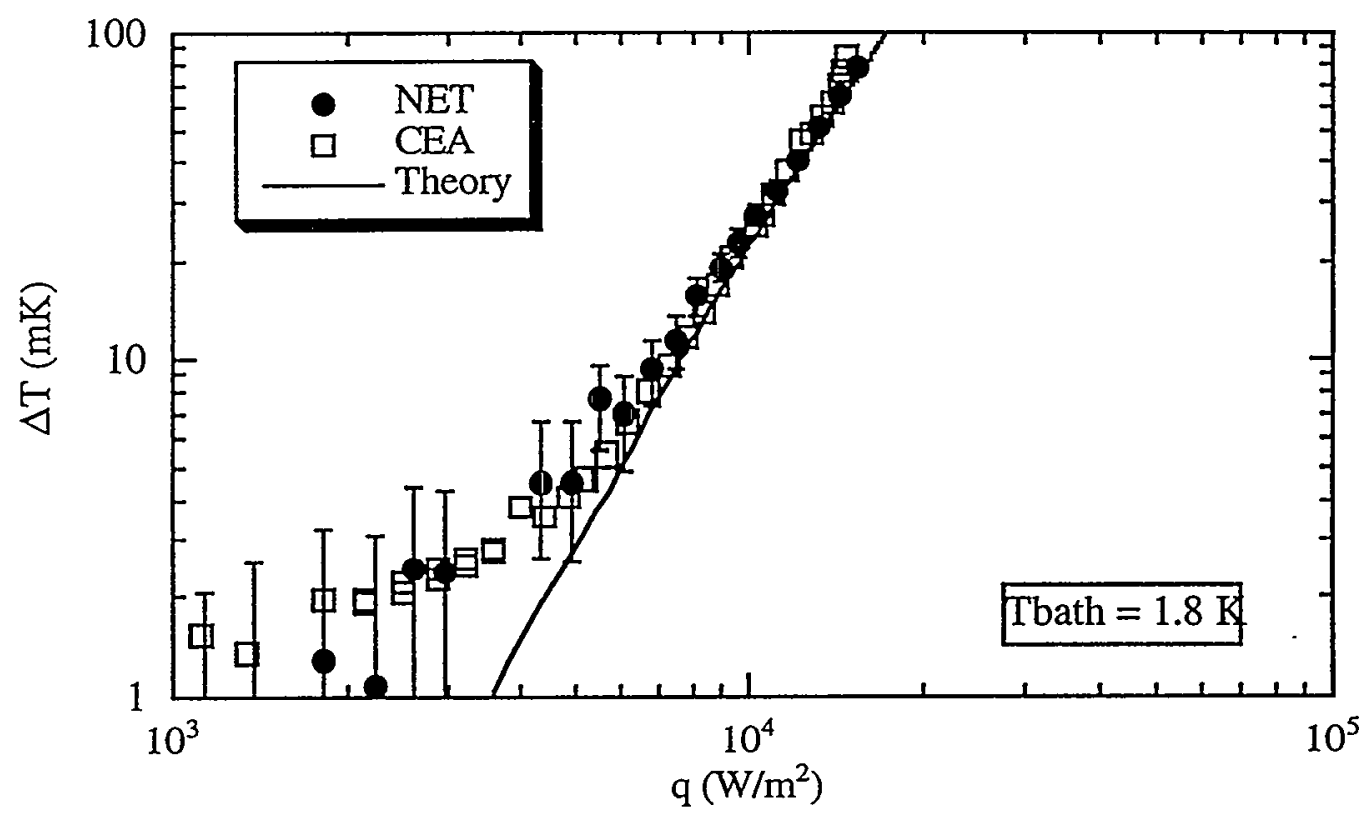

Figure 5.3a. The heat flux vs. temperature difference for $T_{b}=1.8 \mathrm{~K}$.

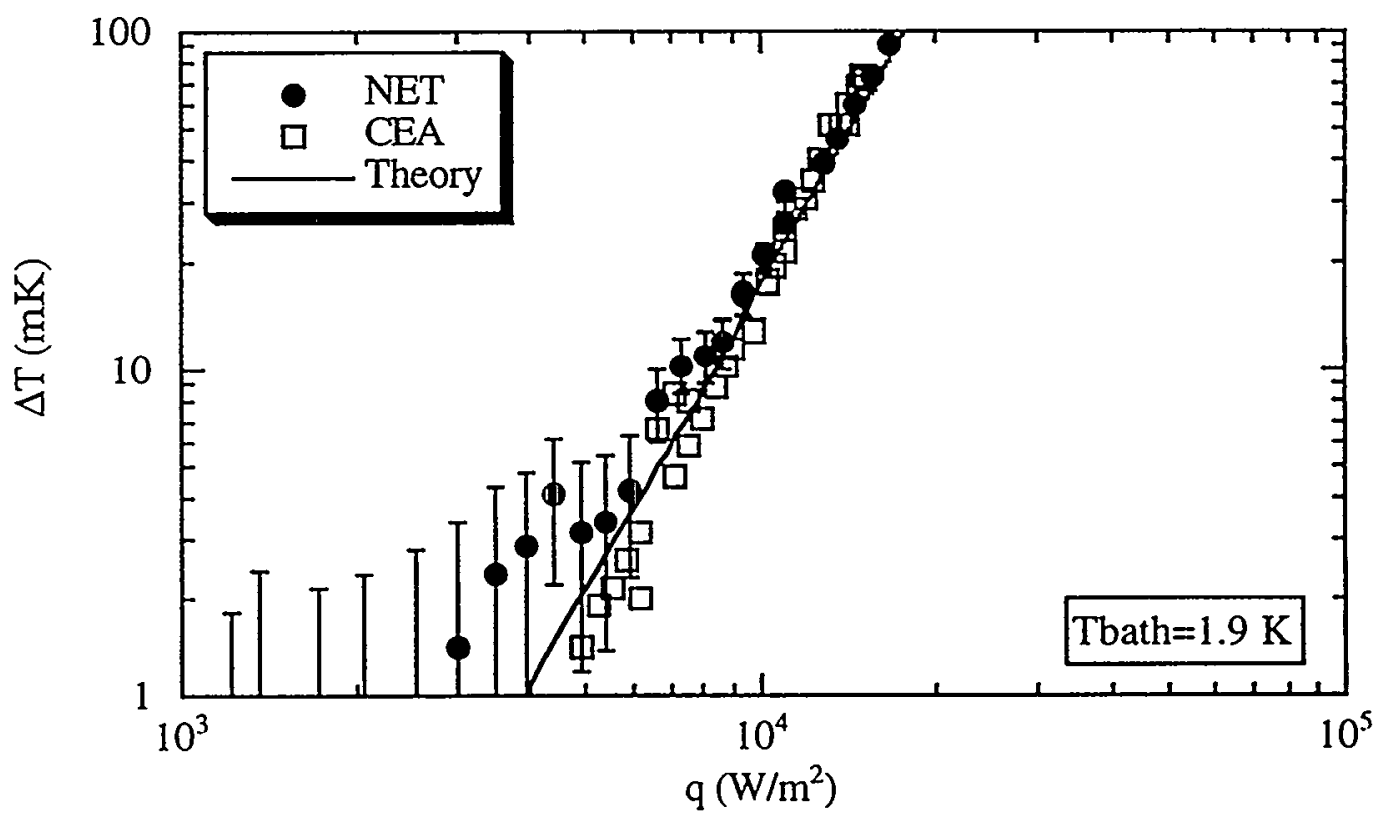

Figure 5.3b. The heat flux vs. temperature difference for $T_{b}=1.9 \mathrm{~K}$. 


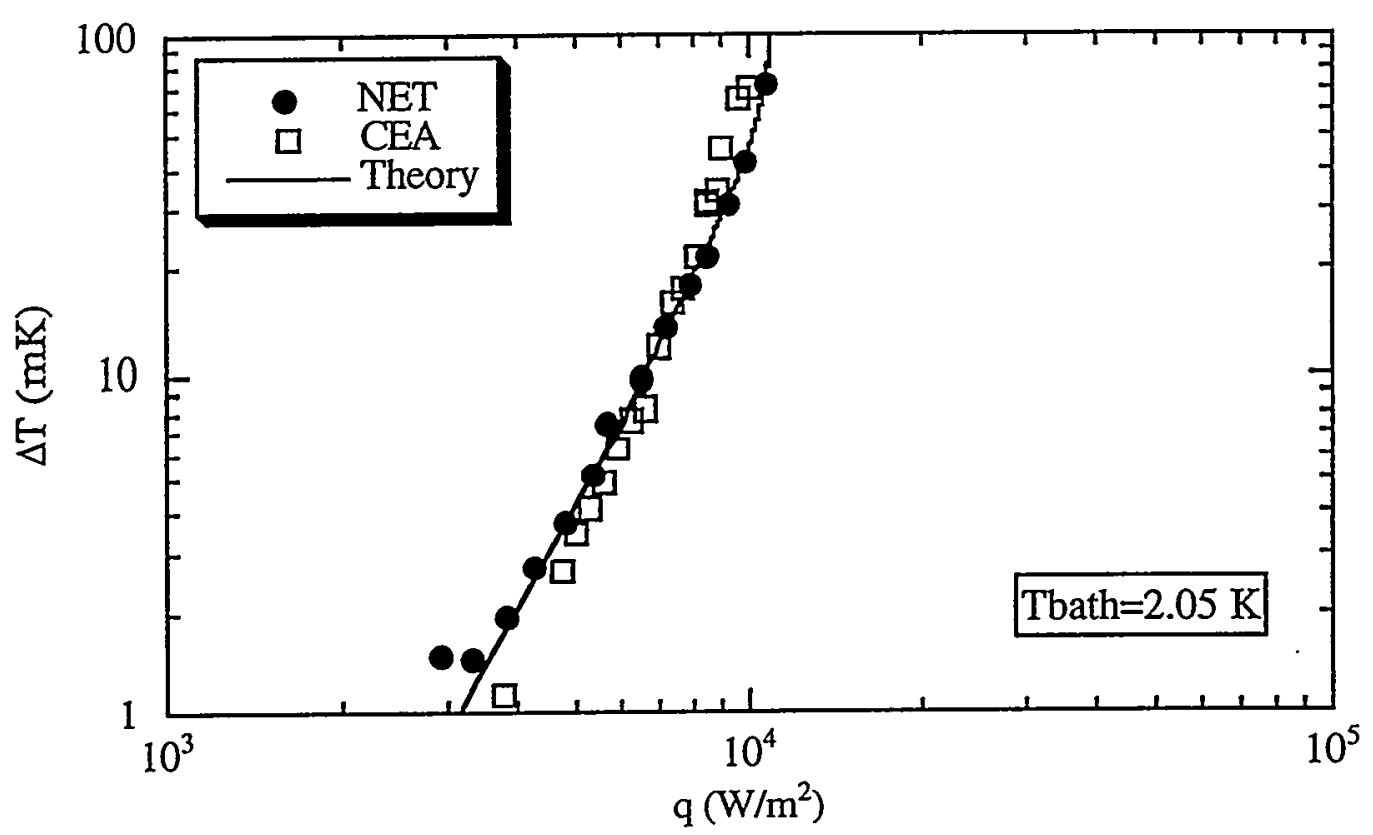

Figure 5.3c. The heat flux vs. temperature difference for $T_{b}=2.05 \mathrm{~K}$.

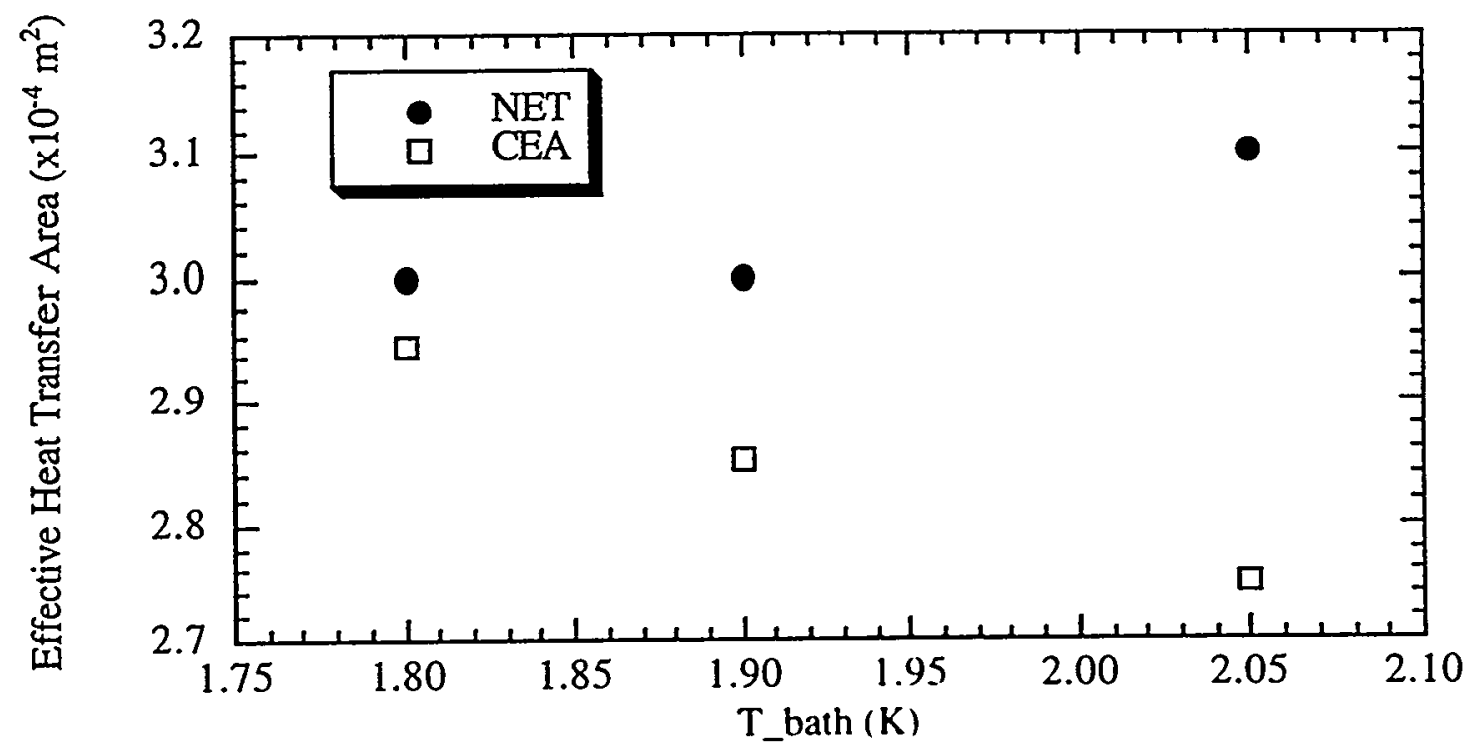

Figure 5.4. The effective heat transfer area as a function of He II temperature. 
Figure 5.4 shows the flow area estimated at different $\mathrm{He}$ II bath temperatures. The effective heat transfer area for both conductor shows different dependence to the bath temperature. However, the effective flow area at $1.8 \mathrm{~K}$ and $1.9 \mathrm{~K}$ is $18 \%$ smaller than the image analysis results. The effective heat transfer area for the CEA conductor is also shows $16 \%$ smaller value than that of image analysis. The heat transfer in He II has some specific geometrical dependence which is not related to the twist pitch or void fraction of the conductor. The reasons that the flow area measured by the counterflow method has a dependence on He II temperature is also unclear.

The flow area estimated by the image analysis and the geometric analysis shows a $10 \%$ difference. This suggests that the helium flow area can be estimated with the nominal strand diameter. However, it is still difficult to estimate the cooled perimeter especially for the CEA conductor which has a helically wrapped stainless steel foil. Although the estimated flow area differences are larger for the counterflow experiment, the maximum and minimum area provides a boundary for the friction factor variation.

\subsection{Pressure Drop Measurements}

Pressure drop measurements were carried out for both prototype NET and CEA Cable-inConduit Conductors, using different fluids to obtain a range of Reynolds numbers. Subcooled liquid nitrogen was used in the low Reynolds number regime. For the high Reynolds number regime, saturated or pressurized He II was used for the NET conductor, while supercritical helium was used for the CEA conductor. The total mass flow rate in the loop was calculated via the pressure drop measured at a venturi flow meter located at the pump outlet. Thermophysical properties of the fluids are estimated with the locally measured temperature and pressure. The experiments were conducted from January 1993 to February 
1994. The results are expressed as a classical correlation of friction factor and Reynolds number, and comparisons made with prominent correlation in turbulent and laminar flow. A model for the CEA conductor is developed based upon the geometry of the flow.passages. The factors which affect the pressure drop of the conductor are discussed.

\subsubsection{NET conductor}

Figure 5.5 shows a typical average He II temperature as a function of mass flow rate in the conductor during the pressure drop measurements. Although increasing the mass flow rate induces frictional heating, the $\mathrm{He}$ II temperature in the loop was maintained around $1.98 \mathrm{~K}$ because of its excellent thermal conductivity and the heat exchanger efficiency. Results of pressure drop measurements with different working fluids are shown in Figure 5.6. Pressurizing the $\mathrm{He}$ II increases the maximum pumping speed (mass flow rate) since cavitation is reduced within the centrifugal pump. The velocity of $\mathrm{He}$ II in the conductor was calculated by,

$$
v=\frac{\dot{m}}{\rho(P, T) A_{\text {flow }, i}}
$$

where $\dot{m}$ is the mass flow rate measured at the venturi, $\rho(P, T)$ is the average density of the fluid within the conductor and $A_{\text {flow, } i}$ is the flow area which was calculated based upon the image analysis result. Figure 5.7 shows the calculated velocity versus pressure drop. 


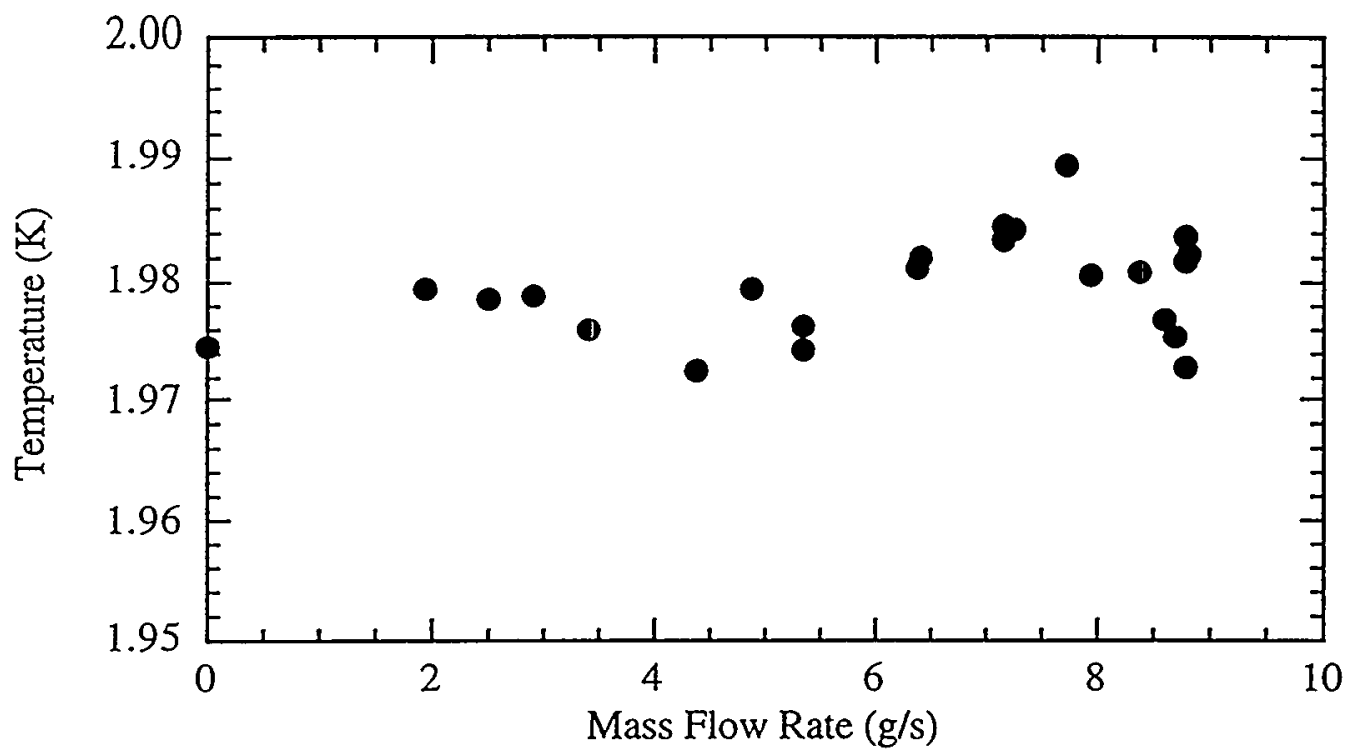

Figure 5.5. He II temperature vs. mass flow rate.

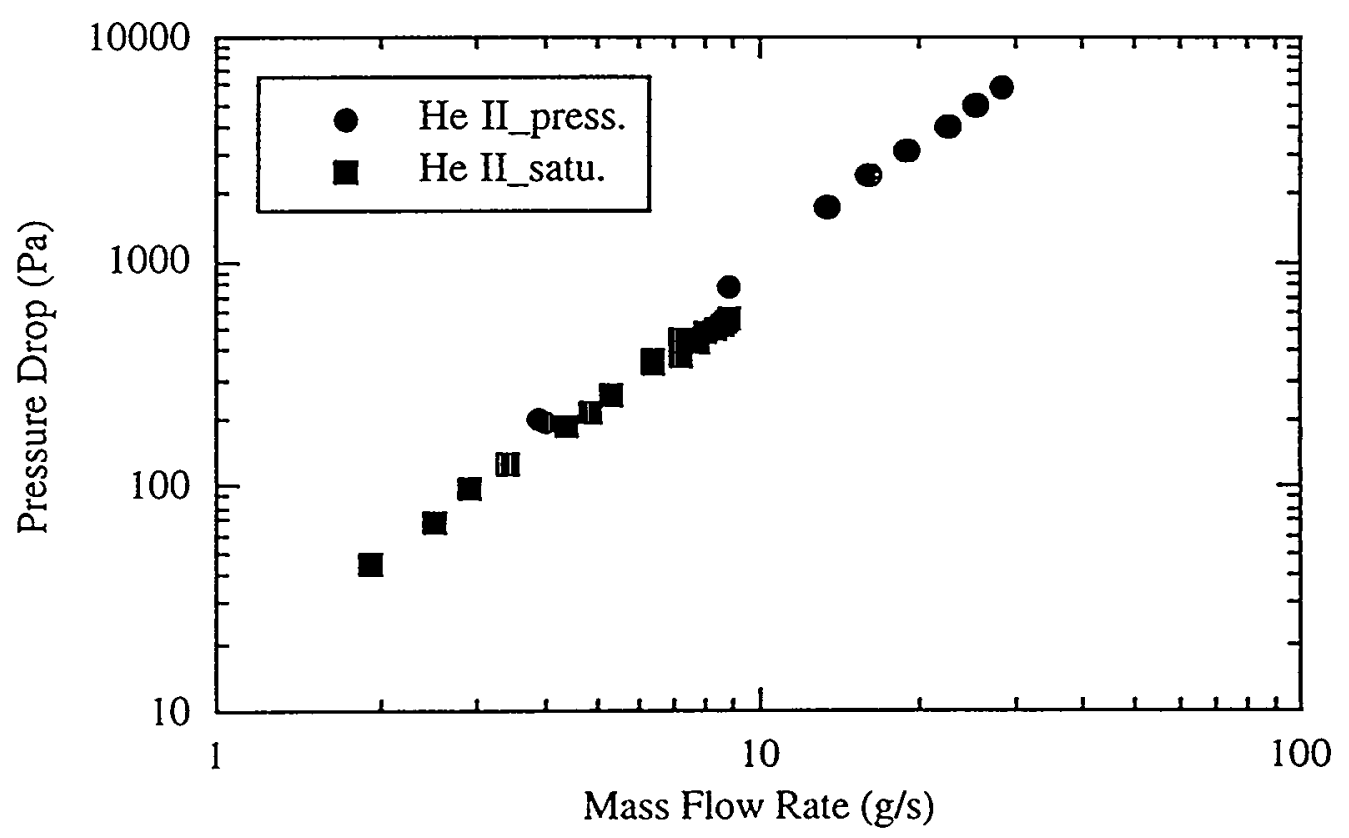

Figure 5.6. Pressure drop vs. mass flow rate 


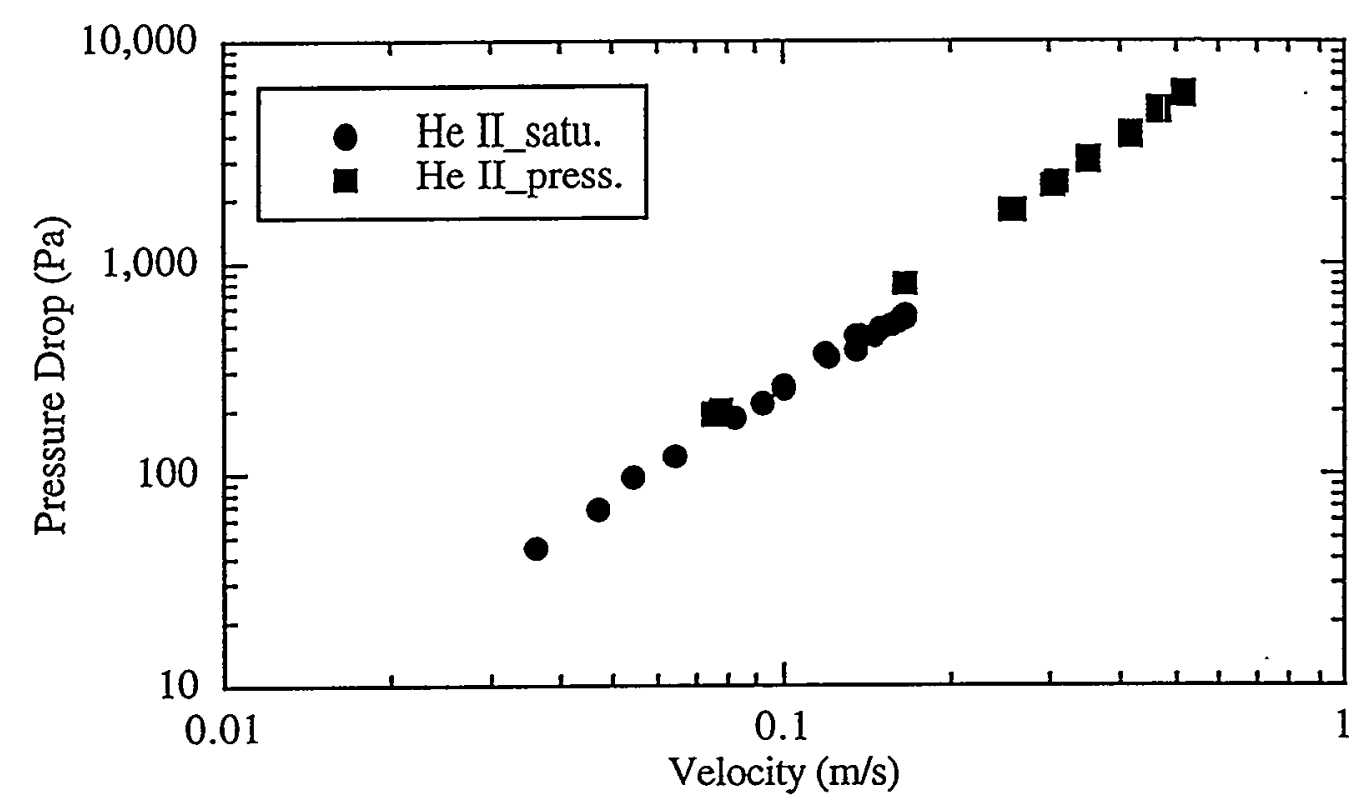

Figure 5.7. Pressure drop vs. average He II velocity.

To correlate the data with a classical relationship for Reynolds number and friction factor, the Reynolds number is calculated from the mass flow rate $\dot{m}$,

$$
R e=\frac{4 \dot{m}}{\eta(P, T) P_{\text {cooled }}}
$$

where $\eta(P, T)$ is the viscosity of the fluid and $P_{\text {cooled }}$ is the cooled perimeter of the conductor. The friction factor is also calculated with,

$$
f=\frac{2 \rho(P, T)}{P_{\text {cuoled }}} \frac{A_{\text {flaw }}^{3}}{\dot{m}^{2}} \frac{\Delta P}{L}
$$


where $L$ is the distance between the differential pressure transducer taps, located at both ends of the conductor.

Figure 5.8 shows the plot of friction factor versus Reynolds number for the conductor containing three different fluids: saturated and pressurized He II, sub-cooled liquid nitrogen. The general correlation for the laminar and turbulent flow for a smooth tube are also plotted. The friction factor of the conductor is higher than that of a smooth tube. Although there is a small gap at Reynolds number $\sim 2000$, no clear transition from laminar to turbulent is seen. This result is typical behavior for CICCs, where turbulent flow is assumed to develop at an early stage of the flow since many twisted strands are tightly packed in the conduit. The solid line represents the Katheder's correlation (3.5) for a void fraction of $47 \%$, which shows adequate agreement with experimental results.

As discussed in the previous section, the comparison of friction factor variation with different flow area analysis is shown in Figure 5.9. Although the computer image analysis gives accurate measurements in both helium flow area and cooled perimeter, the difference between the image analysis and the geometrical component calculation is permissible for engineering applications. The friction factor variation is approximately $6 \%$ at high Reynolds number regime. Thus, it is possible to analyze the flow area of the conductor by simple subtraction of strand area from total area within a conduit. The cooled perimeter can be also calculated by,

$$
P_{\text {coxled }}=\pi D(\# \text { of Strands })+(\text { circumference of the conductor })
$$




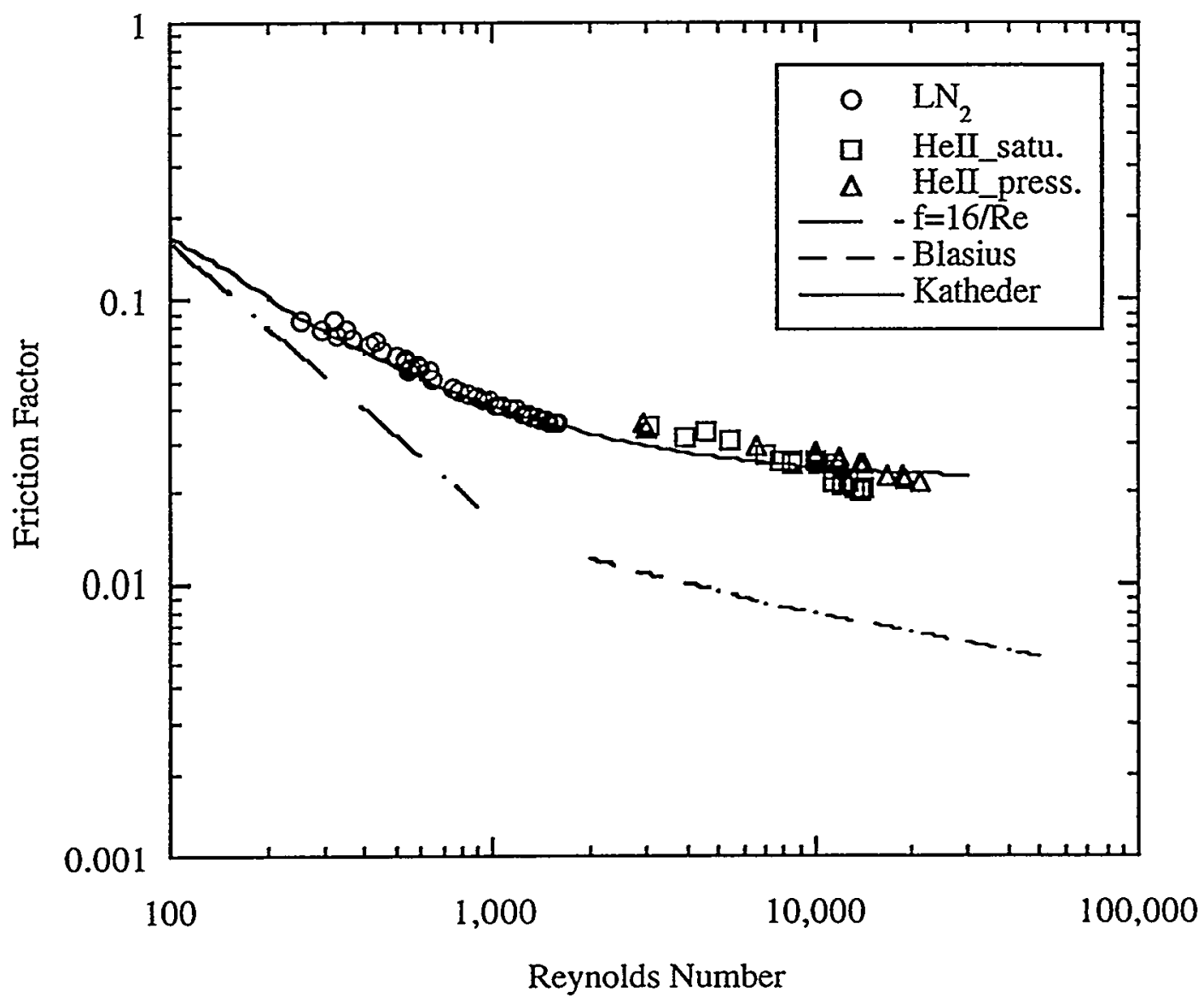

Figure 5.8. Friction factor vs. Reynolds number for the NET conductor. 


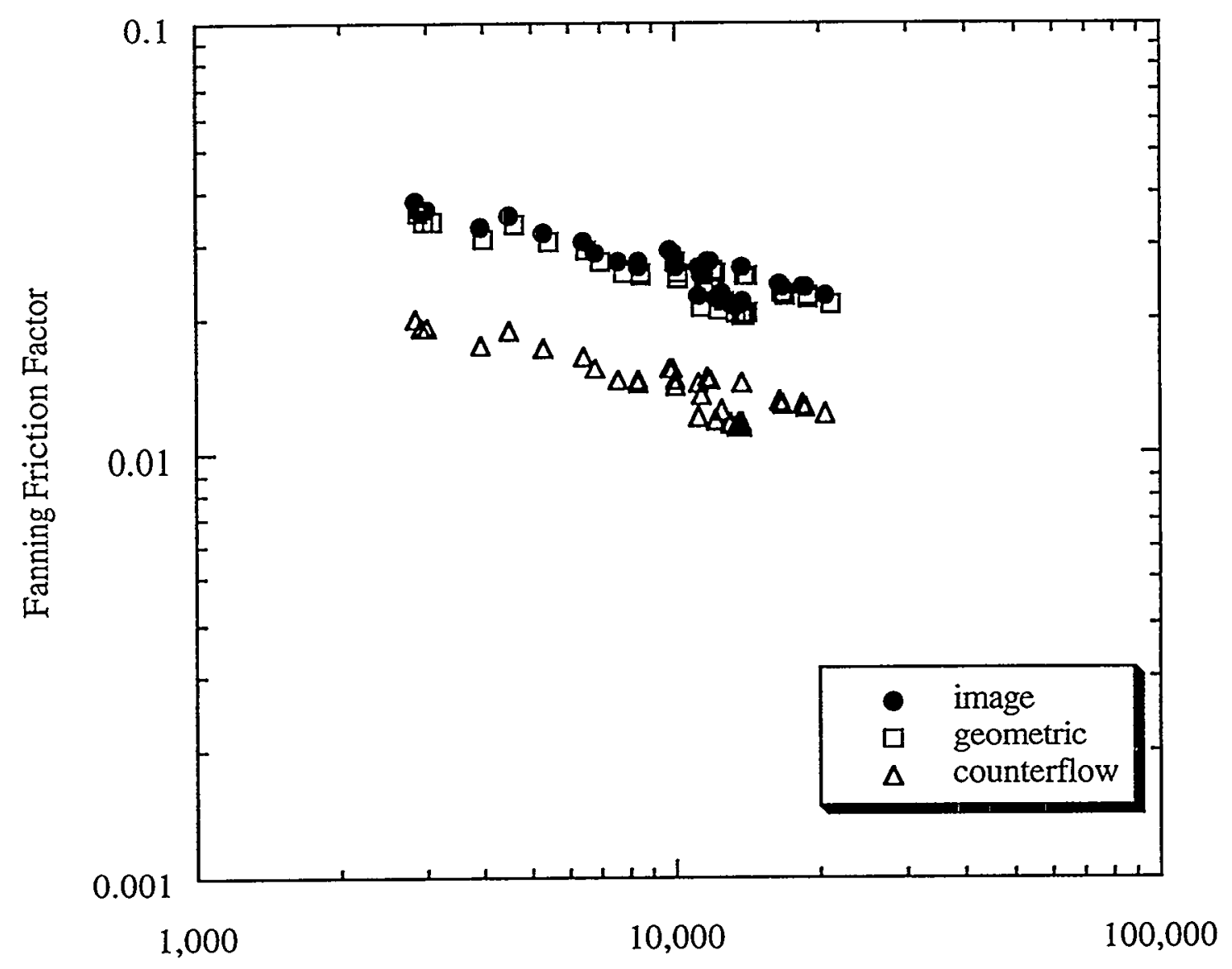

Reynolds Number

Figure 5.9. Comparison of friction factor variation with different flow area analyses. 


\subsubsection{CEA Conductor}

The same experimental procedures were carried out on the CEA conductor. In addition to the overall pressure drop measurements of the conductor, a Pitot tube was located at the down stream end of the conductor to estimate supercritical helium velocity in the central channel. The experiments were conducted in September of 1993 and in February of 1994.

Figure 5.10 shows the average supercritical helium temperature profile in the conductor during the pressure drop measurements.

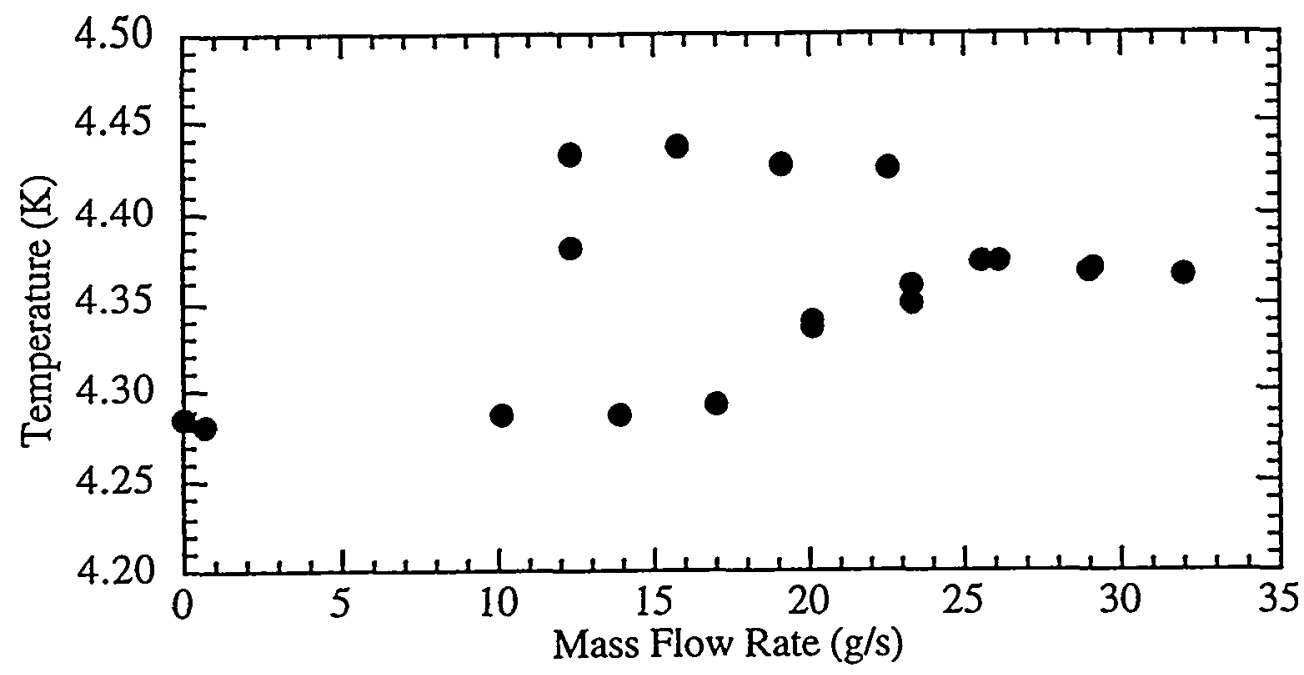

Figure 5.10. Supercritical helium temperature vs. mass flow rate.

The data were taken as a function of pump speed: $0 \mathrm{~Hz}$ to $65 \mathrm{~Hz}$ with $5 \mathrm{~Hz}$ interval and reducing the pump speed to $0 \mathrm{~Hz}$, to check for any hysterisis in the pressure drop measurement. At the beginning of the measurement, the temperature of supercritical helium is relatively high about $4.65 \mathrm{~K}$. This may be caused by the heat leak from the supporing structure of the conductor as well as poor heat transfer at the heat exchanger because of low mass flow rate of supercritical helium. However, increasing the mass flow rate above $20 \mathrm{~g} / \mathrm{s}$ 
apparently improves the heat transfer, and supercritical helium temperature is maintained around $4.3 \mathrm{~K}$. However, the temperature of supercritical helium increases as the mass flow rate exceeds $20 \mathrm{~g} / \mathrm{s}$ due to frictional heating. The same trend is seen in the static pressure in the loop as shown in Figure 5.11.

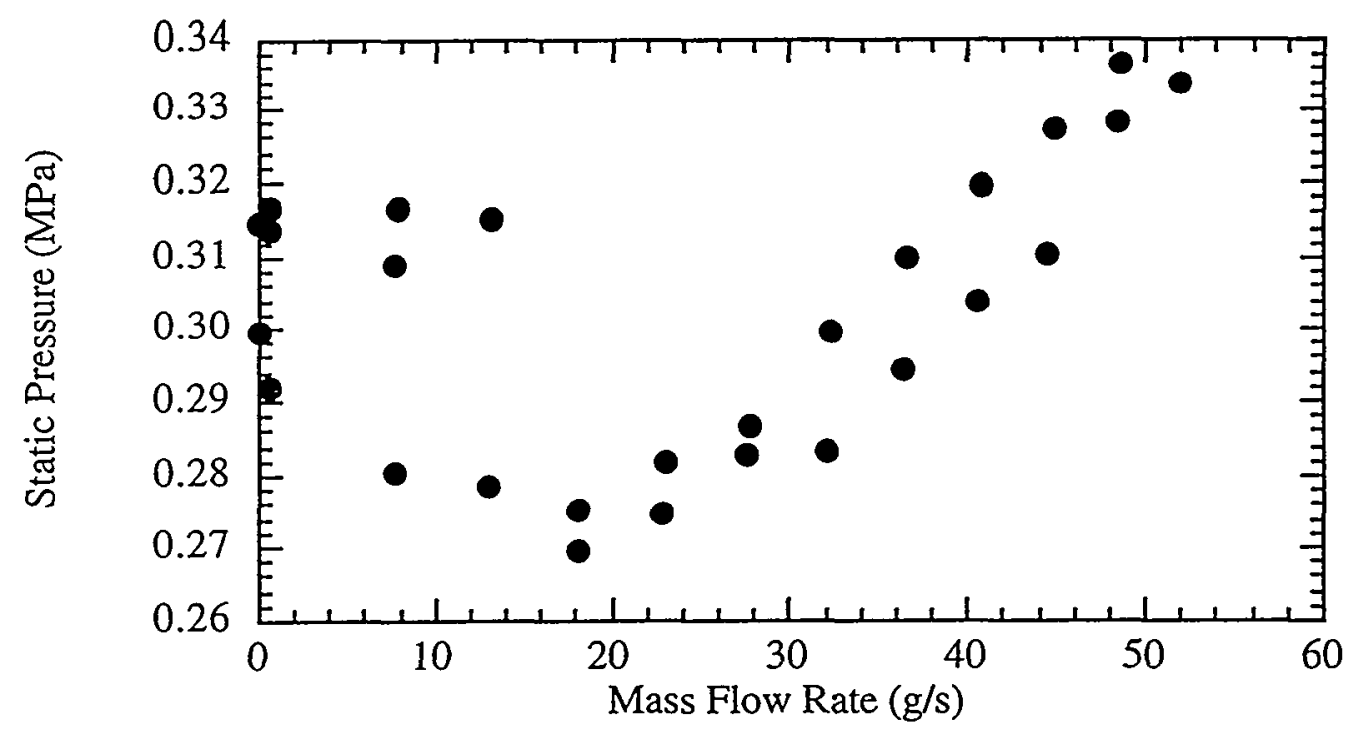

Figure 5.11. Static pressure of supercritical helium in the conductor vs. mass flow rate.

Figure 5.12 shows pressure drop measurements with supercritical helium and liquid nitrogen. The results indicate that the experimental data are reproducible: i.e. no significant error was introduced by the thermal cycling of the system. The velocity at the central channel is calculated from the measured pressure drop at the Pitot tube as,

$$
v=\left(\frac{2 \Delta P}{\rho(P, T)}\right)^{1 / 2}
$$


where the density of supercritical helium is estimated from measured temperature and static pressure at a place $0.02 \mathrm{~m}$ away from the Pitot tube.

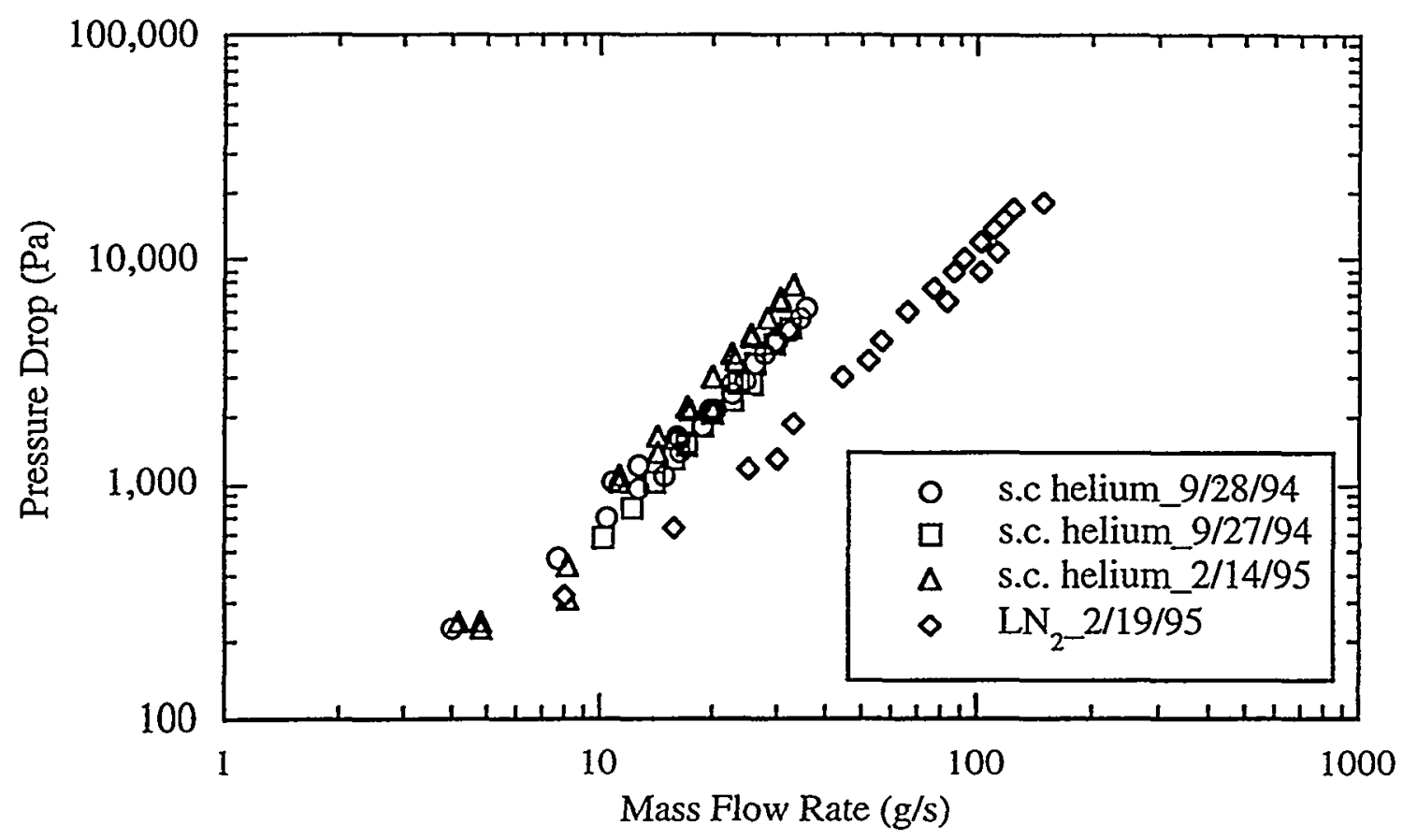

Figure 5.12. Overall pressure drop vs. mass flow rate for different runs.

The average and central channel velocities are plotted as a function of pressure drop along the CICC in Figure 5.13. The helium velocity in the central channel is approximately three times larger than the average velocity for cases where the overall pressure drop of the conductor exceeds $1 \mathrm{kPa}$. Thus, the central channel accomplishes its purpose, reducing the overall flow impedance of the conductor. 


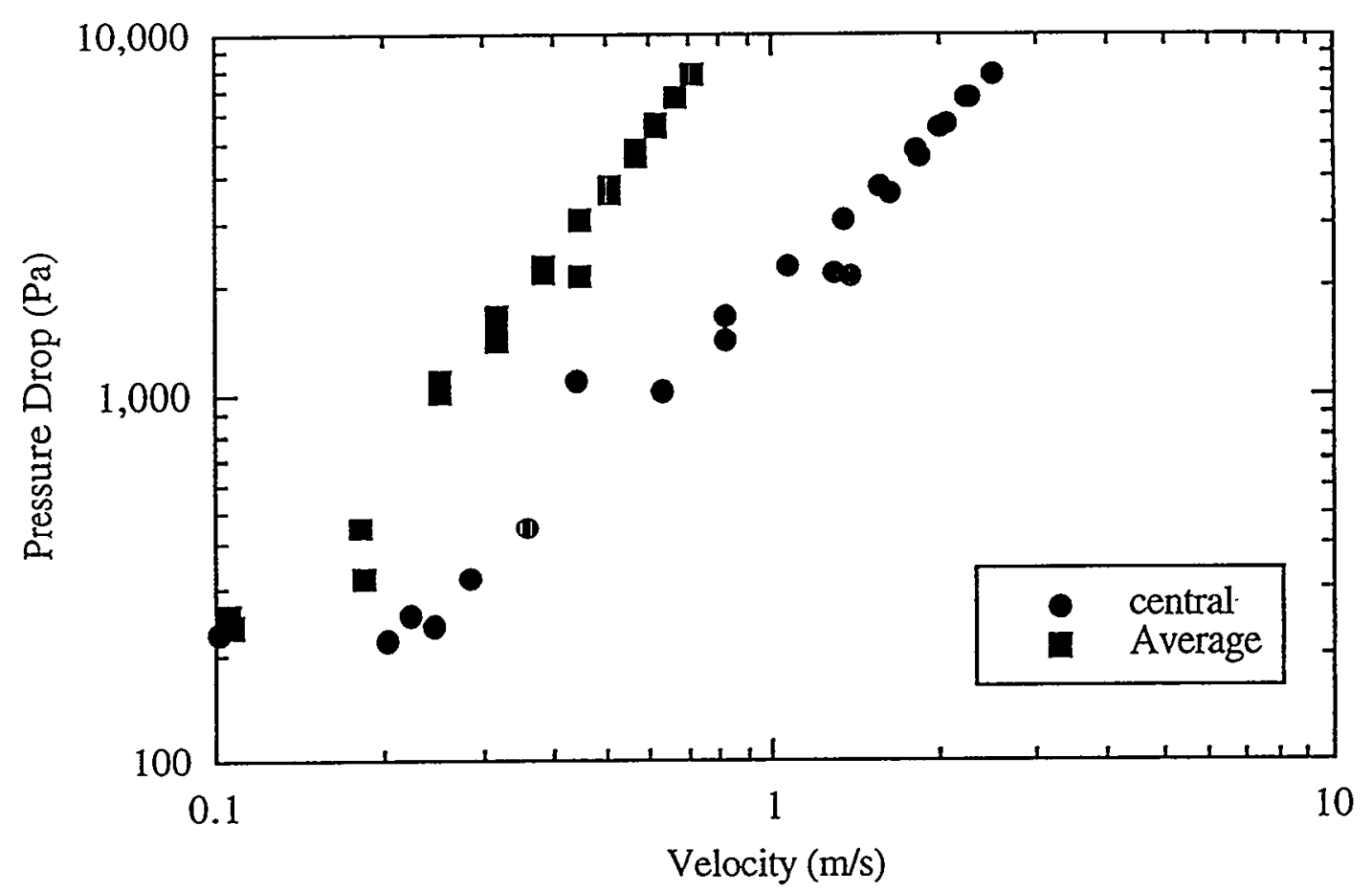

Figure 5.13. Pressure drop vs. supercritical helium velocity.

It is also interesting to plot the fraction of the total helium mass flow rate in the central channel, based on the relationship:

$$
\dot{m}=A_{\text {censral. } i}\left(2 \rho \Delta P_{\text {Pitot }}\right)^{1 / 2}
$$

where the central channel area is estimated by computer image analysis. Figure 5.14 shows fraction of mass flow rate in the central channel as a function of the overall pressure drop. It is clear that the distribution of mass flow varies with the total mass flow rate approaching a plateau when the pressure drops exceeding $2 \mathrm{kPa}$. Scatter in the data points at low mass flow rate regime may be caused by difficulty in making small pressure drop measurements. 


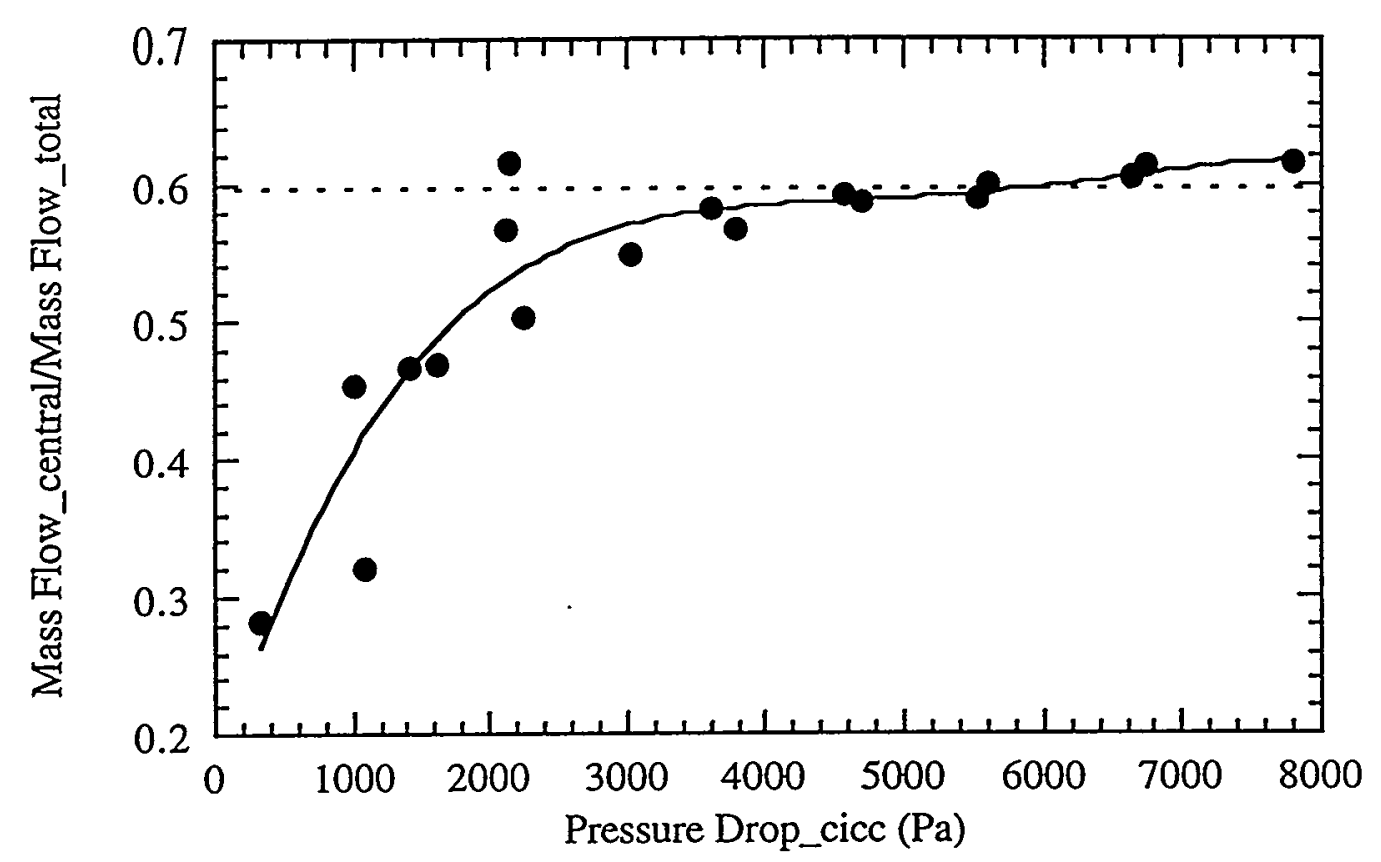

Figure 5.14. Fraction of mass flow rate in the central channel vs. overall pressure drop.

The average velocity in the bundle and external channels may be estimated from,

$$
v=\frac{\dot{m}_{\text {sotal }}-\dot{m}_{\text {central }}}{\rho\left(A_{\text {flow }}-A_{\text {central }, i}\right)}
$$

Figure 5.15 shows a comparison of the supercritical helium velocity in the central channel with there in the bundles and external channels. As the mass flow rate increases to its maximum value, supercritical helium velocity in the central channel is about an order of magnitude larger than the velocity in the rest of conductor. 


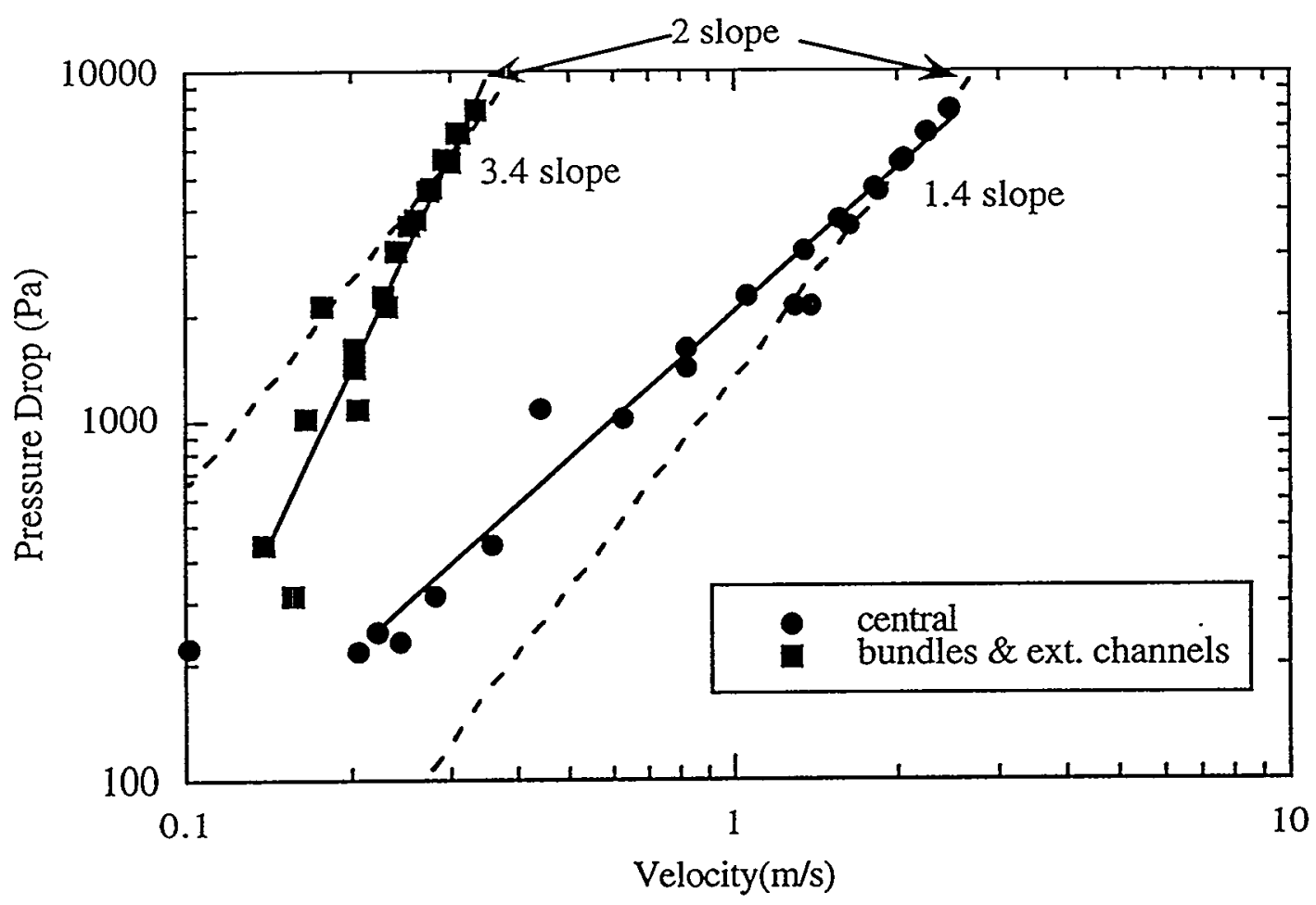

Figure 5.15. Comparison of supercritical helium velocity in the central channel and the rest of the conductor.

Note that the overall pressure drop is not proportional to the velocity squared in the central channel. The slope is close to 1.4 with including the low mass flow rate regime: $\Delta \mathrm{P}<2000$ $\mathrm{Pa}$. However, the slope becomes close to 2 with increasing the mass flow rate. This difference is primarily caused by the measuring a small pressure difference at the venturi flow meter as discussed in the fraction of mass flow rate in the central channel.

Although the area at the central channel is only $20 \%$ of the total flow area, approximately $60 \%$ of the total mass flow rate is through the central channel. A comparison of the hydraulic diameters for the total conductor and the central channel clearly indicates why the mass flow rate at the central channel is very high. The hydraulic diameter of central channel may be calculated from, 


$$
D_{h}=\frac{4 A_{\text {central }, i}}{P_{\text {cooled }, i}}
$$

where the cooled perimeter is estimated from the image analysis. Figure 5.16 shows calculated hydraulic diameter and the cross section for both the central channel and the total conductor. Although the total flow area of the conductor is much higher than that of the central channel, the hydraulic diameter is actually smaller than that of the central channel by almost an order of magnitude : $D_{h, \text { central }}=4.0 \mathrm{~mm}$ and $D_{h, \text { toral }}=0.6 \mathrm{~mm}$. This is due to the large cooled perimeter in the bundle and external channels.

Hydraulic Diameter for
the Central Channel

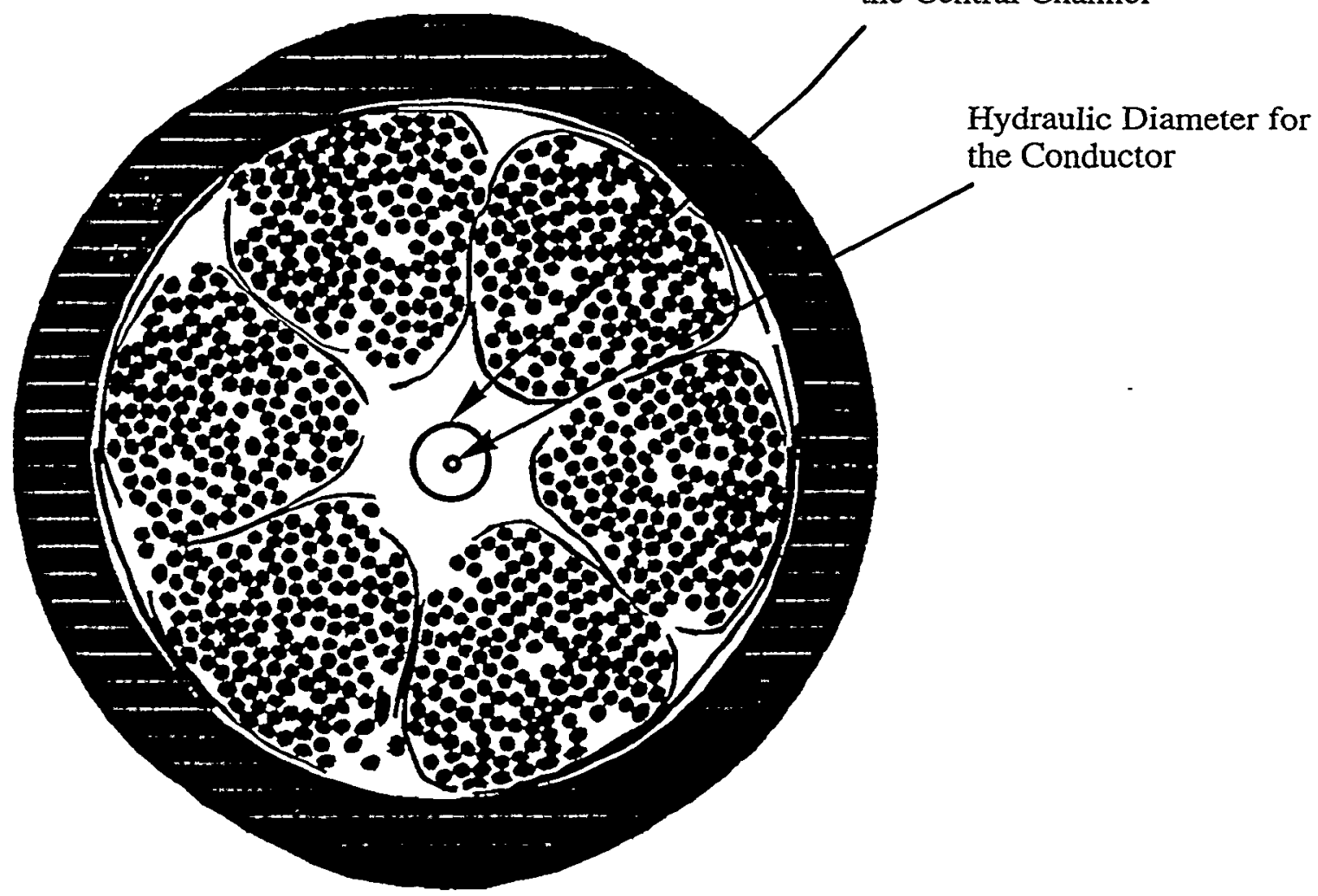

Figure 5.16. Hydraulic diameter of the central channel and the conductor.

The data shows that the central channel of the conductor has a large influence on the overall flow characteristics. The shape of the central channel is similar to the six point star 
duct shown in Figure 5.17. The hydraulic diameter of such a channel is smaller than the duct diameter because of the large cooled perimeter. This suggests that the friction factor for the duct may be smaller than that of an equivalent tube. Shih ${ }^{46}$ and Gunn and Darling 47 derived an analytic solution for the star ducts in laminar flow. The solution suggests that the friction factor at low Reynolds number can be expressed as,

$$
f=\frac{6.6}{R e}
$$

They also found an empirical friction factor correlation for the star ducts at high Reynolds number that is somewhat smaller than calculated from the Blasius correlation.

In the case of CEA conductor, the central channel has irregular boundaries. Thus, the Blasius correlation is used to estimate the friction factor variation of a central channel for modeling the overall conductor.

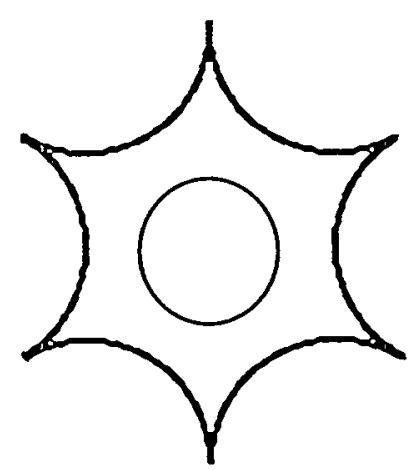

Figure 5.17. Six point star duct with a hydraulic diameter.

The Reynolds number and friction factor are calculated, using Equations (5.3) and (5.4). Figure 5.18 shows the friction factor versus Reynolds number for four sets of data. In addition, correlation for laminar flow in the star ducts and turbulent flow in the smooth tube are plotted. Friction factor variation for the turbulent regime is within $30 \%$ of the Blasius correlation. Since the hydraulic characteristics of the conductor are dominated by the central 
channel, one might expect clear transition from laminar to turbulent flow. However, the transition is apparently smoothed because the central channel is not isolated from the surrounding bundles. The non-uniformity near the boundary between the channels must have significant effects. Since there is a gap in the helically wound stainless strip at the interface, the flow in the central channel and in the bundle are mixed. Thus, the communication of the helium flow in the central channel and bundles induce the smooth transition of the flow. Having a central channel in the conductor largely reduces the flow impedance of the CICC and the hydraulic characteristics at high Reynolds number regime can be described by Blasius correlation.

A comparison of different flow area analyses is shown in Figure 5.19. The image analysis and counter flow analysis results give upper and lower bound of the friction factor variation. The variation with different flow area analysis is approximately $45 \%$. However, the variation between the image analysis and the simple calculation is only $12.5 \%$, which is adequate agreement for the engineering application. 


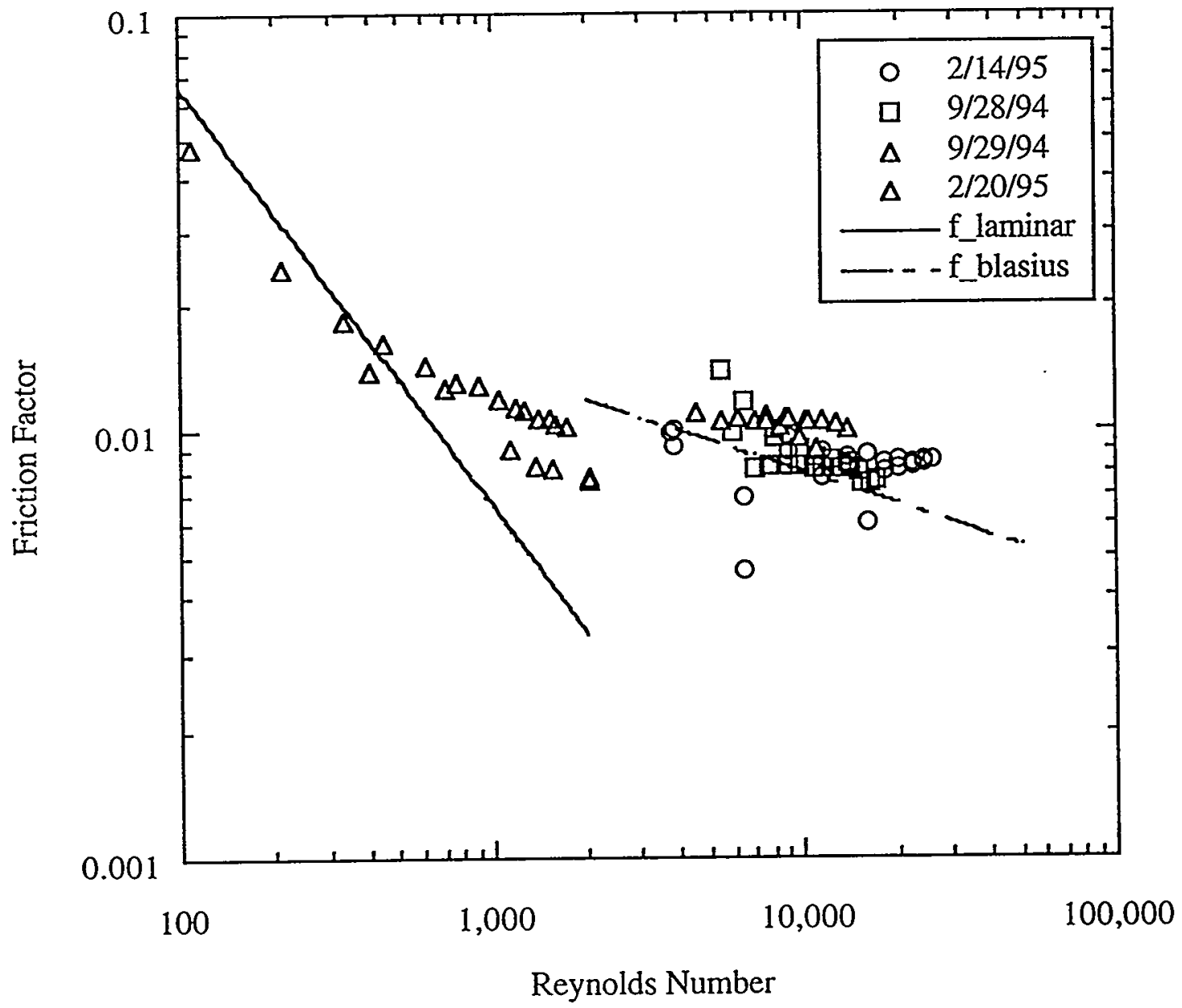

Figure 5.18. Friction factor vs. Reynolds number for the CEA conductor. 


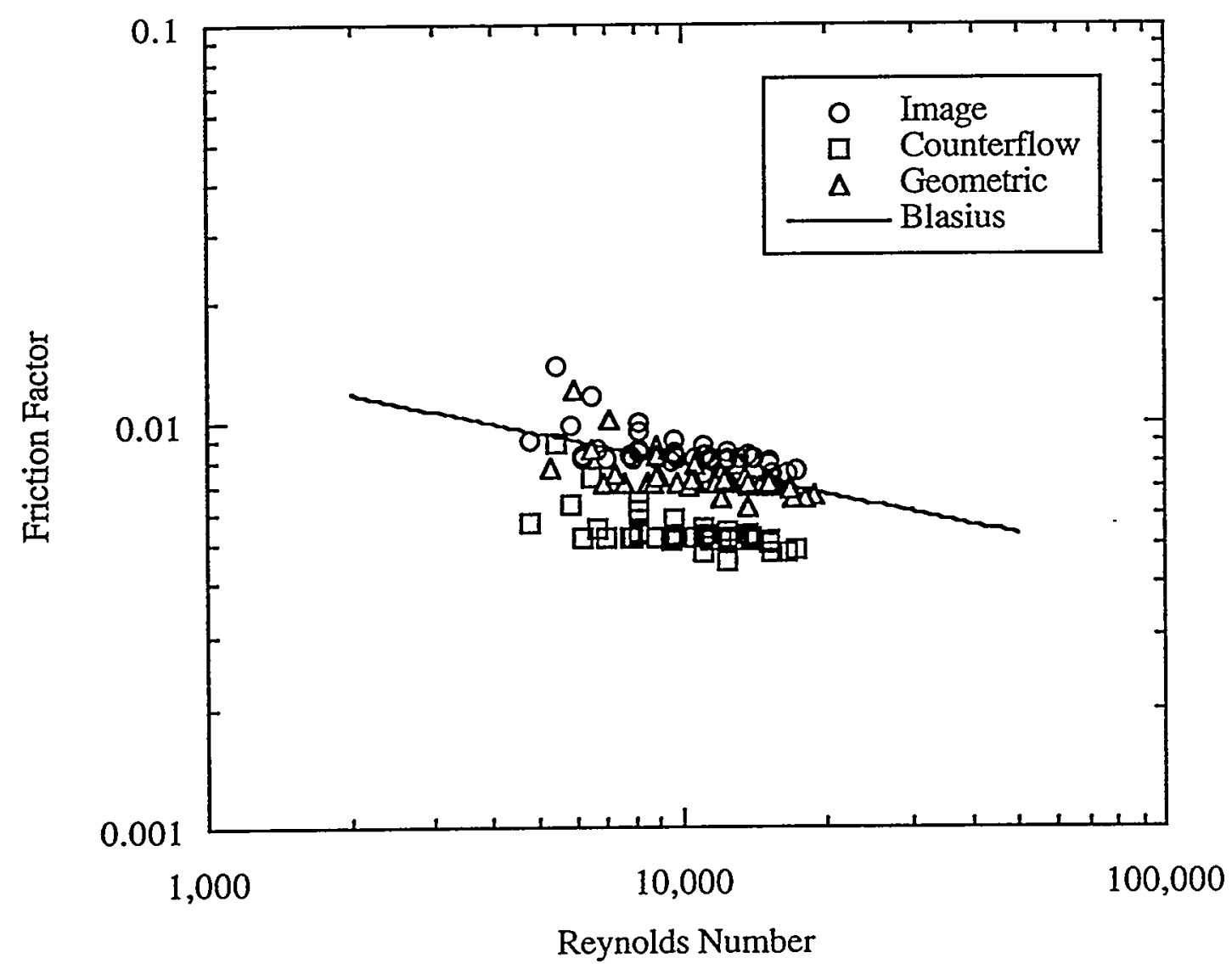

Figure 5.19. Comparison of different flow area analysis on friction factor variation.

\subsubsection{Modeling Flow in the CEA conductor}

A flow model for the CEA conductor is developed based upon parallel independent flow passages: a central channel, six bundles and six external channels. Computer image analysis estimates the flow area as well as the cooled perimeter for each channel. Figure 5.20 and Table 5.3 show the results. The total flow area is less than the flow area estimated by image analysis since each bundle is covered with imaginary stainless steel foil and small gaps 
between the conduit and outer stainless steel strip for the bundles are neglected in the model. On the other hand, total cooled perimeter is increased because some portion of the cooled perimeter overlaps with different flow passages.

\begin{tabular}{ccccc}
\hline Channel & Area $\left(\mathrm{cm}^{2}\right)$ & $\begin{array}{c}\text { Pcooled } \\
\text { w/o strands }\end{array}$ & $\begin{array}{c}\text { void fraction } \\
\text { image (\%) }\end{array}$ & $\begin{array}{c}\text { void fraction } \\
\text { geometric } \\
(\%)\end{array}$ \\
\hline Central & 0.580 & 5.902 & & \\
\hline bundle_1 & 0.954 & 3.623 & 41.1 & 36.8 \\
\hline bundle_2 & 0.975 & 3.794 & 42.6 & 38.2 \\
\hline bundle_3 & 0.916 & 3.544 & 38.9 & 34.2 \\
\hline bundle_4 & 0.950 & 3.591 & 41.2 & 36.6 \\
\hline bundle_5 & 0.943 & 3.675 & 40.8 & 36.1 \\
\hline bundle_6 & 0.945 & 3.601 & 40.8 & 36.2 \\
\hline ext_1 & 0.069 & 2.368 & & \\
\hline ext_2 & 0.066 & 2.623 & & \\
\hline ext_3 & 0.066 & 2.333 & & \\
\hline ext_4 & 0.040 & 1.622 & & \\
\hline ext_5 & 0.072 & 2.937 & & \\
\hline ext_6 & 0.040 & 1.569 & & \\
\hline strip & 0.576 & & & \\
\hline total & 7.162 & & \\
\hline
\end{tabular}

Table 5.3

The strands area for each bundle is calculated from the average strand diameter determined by image analysis.

$$
A_{\text {strands }}=\frac{\pi D^{2}}{4} \text { (\# of strands in the bundle) }
$$




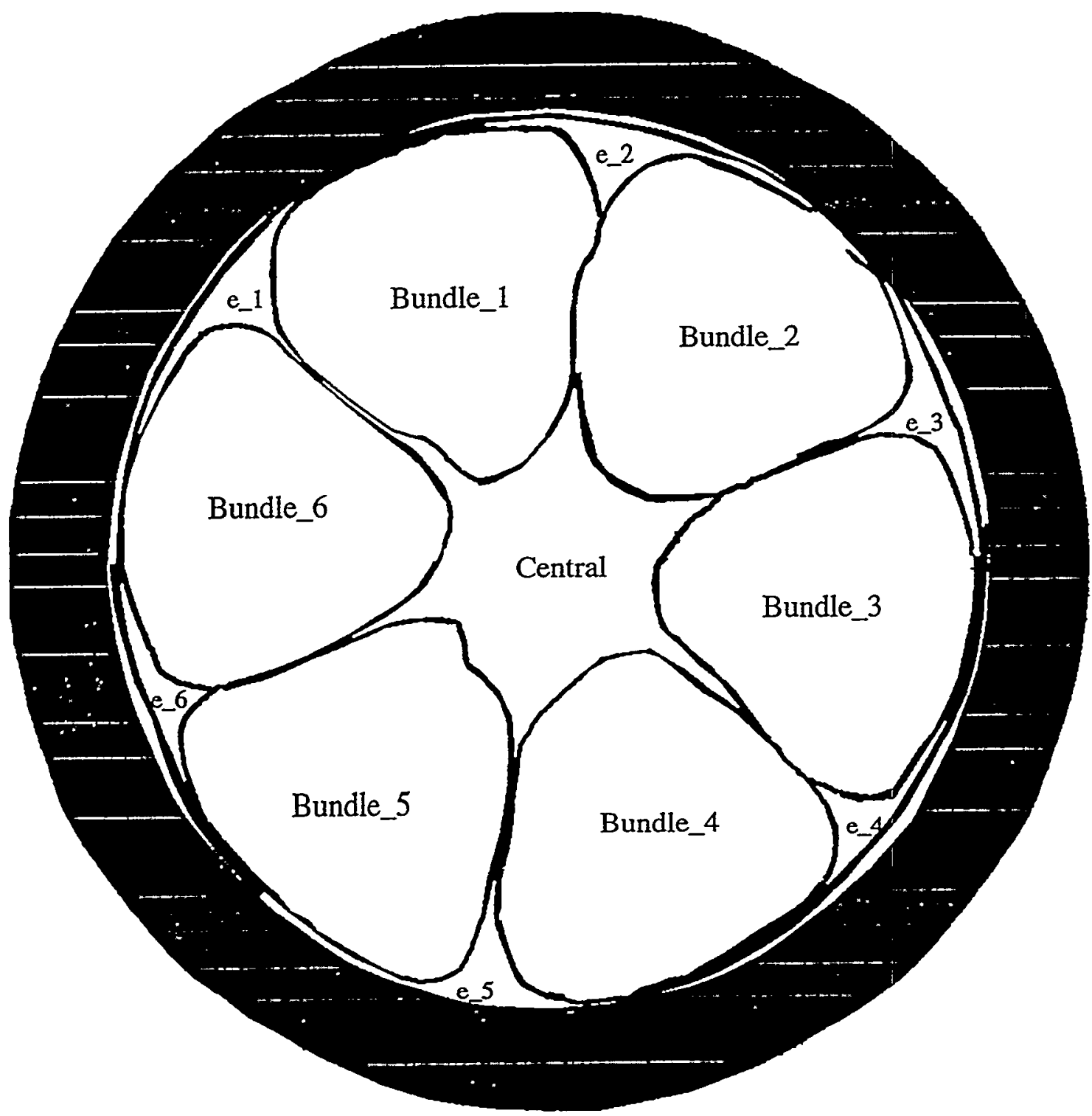

Figure 5.20. Cross section for the flow model of CEA conductor.

According to the image analysis, the average strand diameter is $0.703 \mathrm{~mm}$. The specification from CEA is for the nominal diameter of the strands to be $0.73 \mathrm{~mm}$. These two different diameters were used to calculate the void fraction as well as cooled perimeter of the bundles, and their impact on subsequent analyses is discussed below.

The flow along the conductor can be characterized by three different flow regions: a central channel; six bundles and six external channels. To calculate the friction factor for 
each channel, two different correlations were used. The Blasius correlation was used for the central channel and six external channels since these are some modification of the star ducts configuration, while Katheder's correlation was used to estimate the friction factor for the six bundles. With these two correlations, the friction factor can be calculated as a function of Reynolds number since the mass flow rate can be written as,

$$
\dot{m}=\left(\frac{2 \rho}{P_{\text {cooled }}} \frac{\Delta P}{L} \frac{A_{\text {flow }}^{3}}{f}\right)^{1 / 2}
$$

Modifying Equation (5.1) with Blasius correlation leads to

$$
\dot{m}=\left(\frac{2 \rho}{P_{\text {cooled }}} \frac{\Delta P}{L} \frac{A_{\text {flow }}^{3}}{0.079\left(\frac{4 m}{\eta P_{\text {cooled }}}\right)^{-0.25}}\right)^{1 / 2}
$$

Solving Equation (5.2) numerically as a function of the pressure drop gives the mass flow rate for each channel. The fitting parameter for each channel is the pressure drop across the channel since all channels have the same pressure drop for a given mass flow rate. Similarly, the mass flow rate for the six bundles are calculated by combined Equations (5.12) with Katheder's correlation (3.5),

$$
\dot{m}=\left(\frac{2 \rho}{P_{\text {couled }}} \frac{\Delta P A_{\text {flow }}^{3}}{L} / \frac{1}{4 v_{\text {void }}^{0.72}}\left(\frac{19.5}{\left(\frac{4 \dot{m}}{\eta P_{\text {cooled }}}\right)^{0.88}}+0.051\right)\right)^{1 / 2}
$$


All parameters in the above equations are calculated for each channel. For the mass flow rate calculation at the bundles, parameters are calculated based upon either image analysis or the geometrical component analysis.

Figure 5.21 shows the calculation results for the mass flow rate and pressure drop of different channels. The total mass flow rate in the conductor was determined by,

$$
\dot{m}_{\text {total }}=\dot{m}_{\text {central }}+\sum_{i=1}^{6} \dot{m}_{i, \text { bundle }}+\sum_{i=1}^{6} \dot{m}_{i, \text { ext_channel }}
$$

According to the results based upon the geometrical component calculation, approximately $65 \%$ of the total mass flow is through the central channel of the conductor. The image analysis calculation gives approximately $60 \%$ of the total mass flow rate in the central channel. The total mass flow rate in the bundle region varies ten percent difference between the calculation based on the image analysis and the geometric component analysis since the flow area in the bundle region is about ten percent larger for the image analysis result than in the geometric component analysis.

The total mass flow rate from pressure drop measurements is compared with two models as shown in Figure 5.22. The geometrical component analysis shows fairly good agreement with experimental results. Since the image analysis gives a larger total flow area than the geometric component analysis, the total mass flow rates are also higher. 


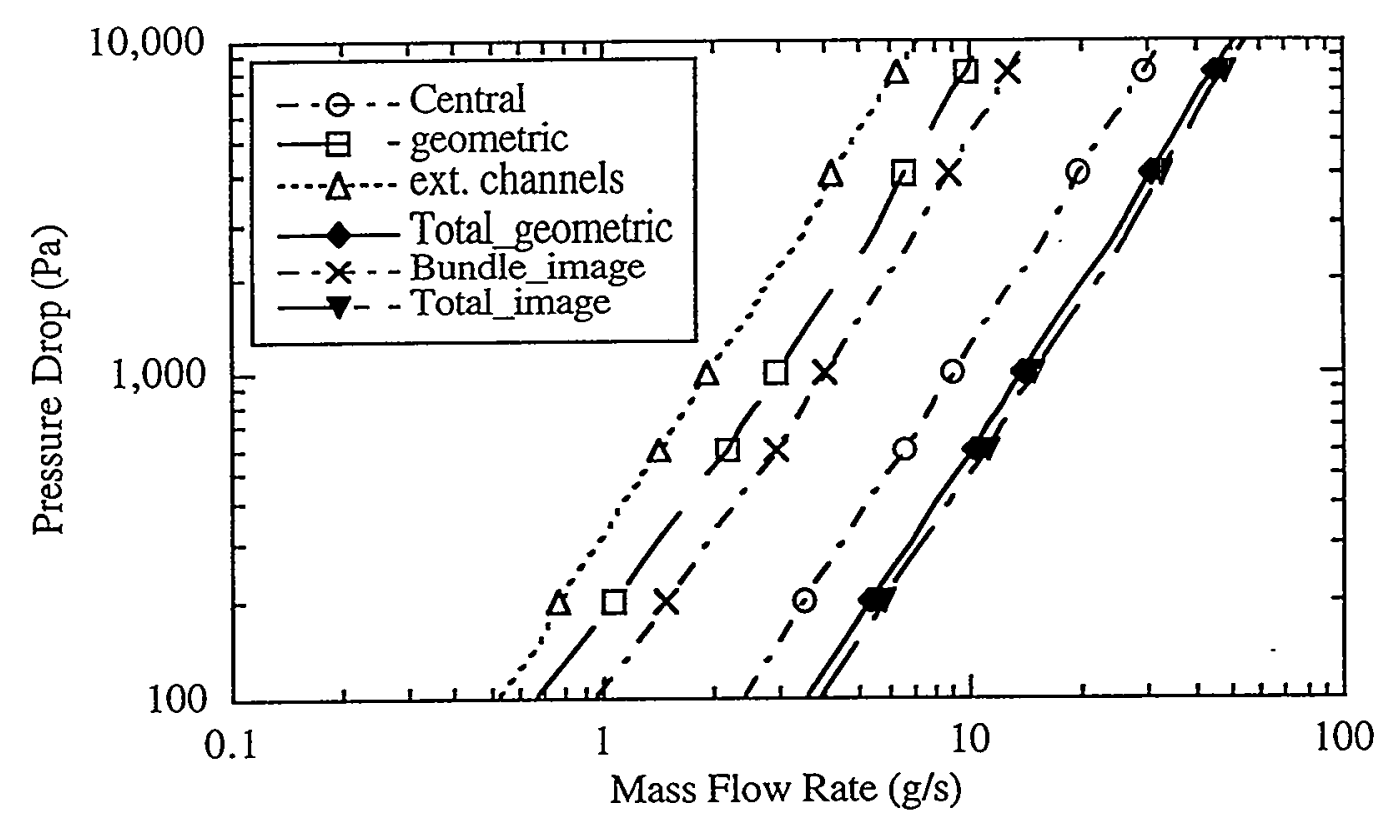

Figure 5.21. Comparison of two models (pressure drop vs. mass flow rate).

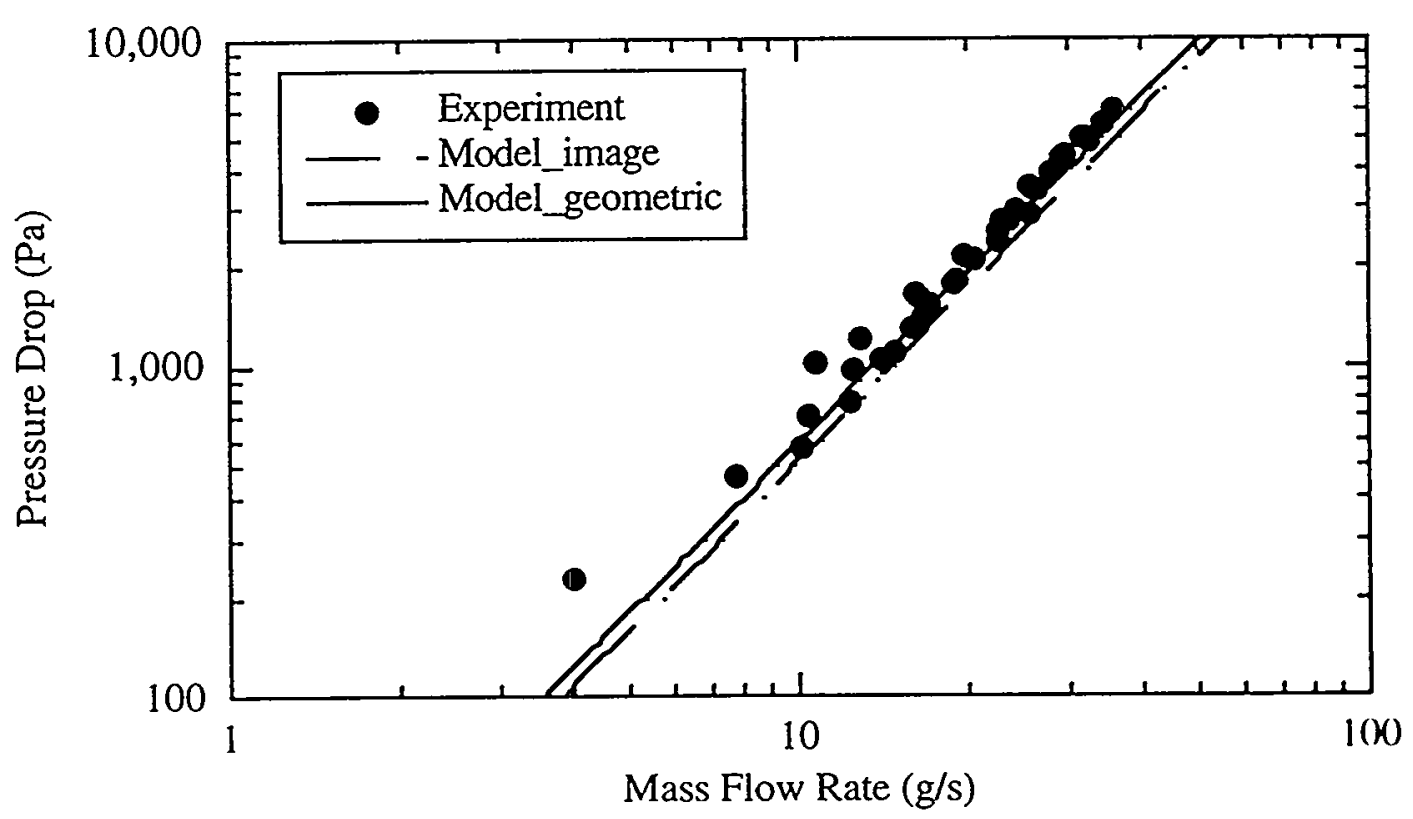

Figure 5.22. Pressure drop vs. mass flow rate (model and experimental results). 
The Reynolds number is calculated, using the total mass flow rate and cooled perimeter of the model,

$$
R e=\frac{4\left(\dot{m}_{\text {central }}+\sum_{i=1}^{6} \dot{m}_{i, \text { bundle }}+\sum_{i=1}^{6} \dot{m}_{i, \text { ext_clannel }}\right)}{\eta\left(P_{\text {cooled,_enural }}+\sum_{i=1}^{6} P_{\text {cooled,bundle }}+\sum_{i=1}^{6} P_{\text {cooled,ext_channel }}\right)}
$$

and the friction factor is calculated from,

$$
f=\frac{2 \rho}{P_{\text {cooled,otal }}} \frac{A_{\text {flow, total }}}{\dot{m}_{\text {lotal }}} \frac{\Delta P}{L}
$$

Figure 5.23 shows the variation of friction factor with Reynolds number for the two models. The friction factor based upon the image analysis shows much smaller Reynolds number than that of the Blasius correlation. On the other hand, the result based upon the geometric component calculation shows very close agreement with the Blasius correlation and with the experimental result.

The flow velocity in the central channel as a function of total pressure drop is shown in Figure 5.24. Apparently, the friction factor variation of the central channel is underestimated by the Blasius correlation. Even though a star duct channel shows lower Reynolds number than a smooth tube, the central channel of the conductor has higher friction factors than for a smooth tube. This is mainly caused by the non uniformity at the interface between the channels, which consists of helically wrapped stainless steel foils and strands. 


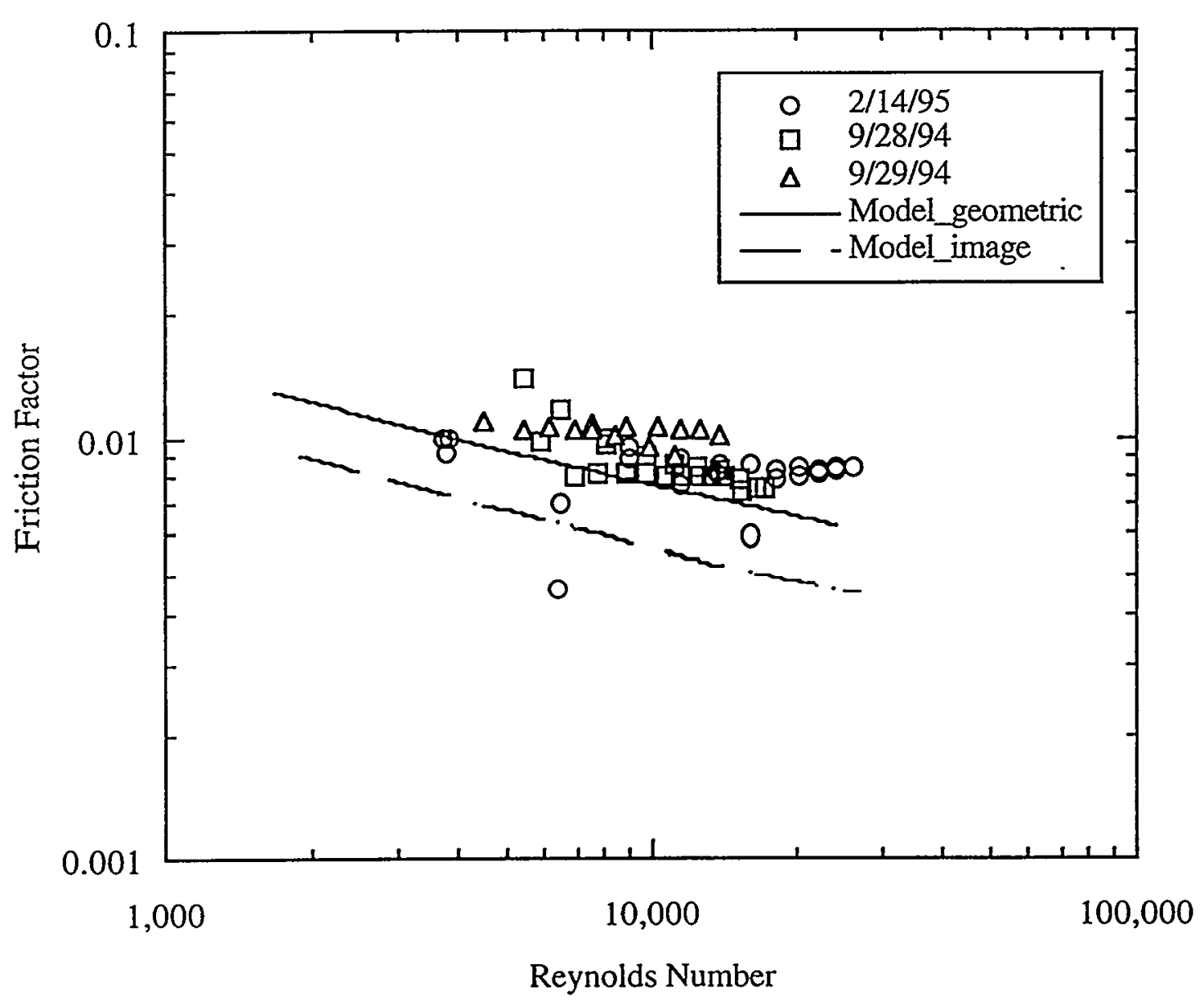

Figure 5.23. Comparison of a flow model with experimental results in the friction factor vs. Reynolds number. 


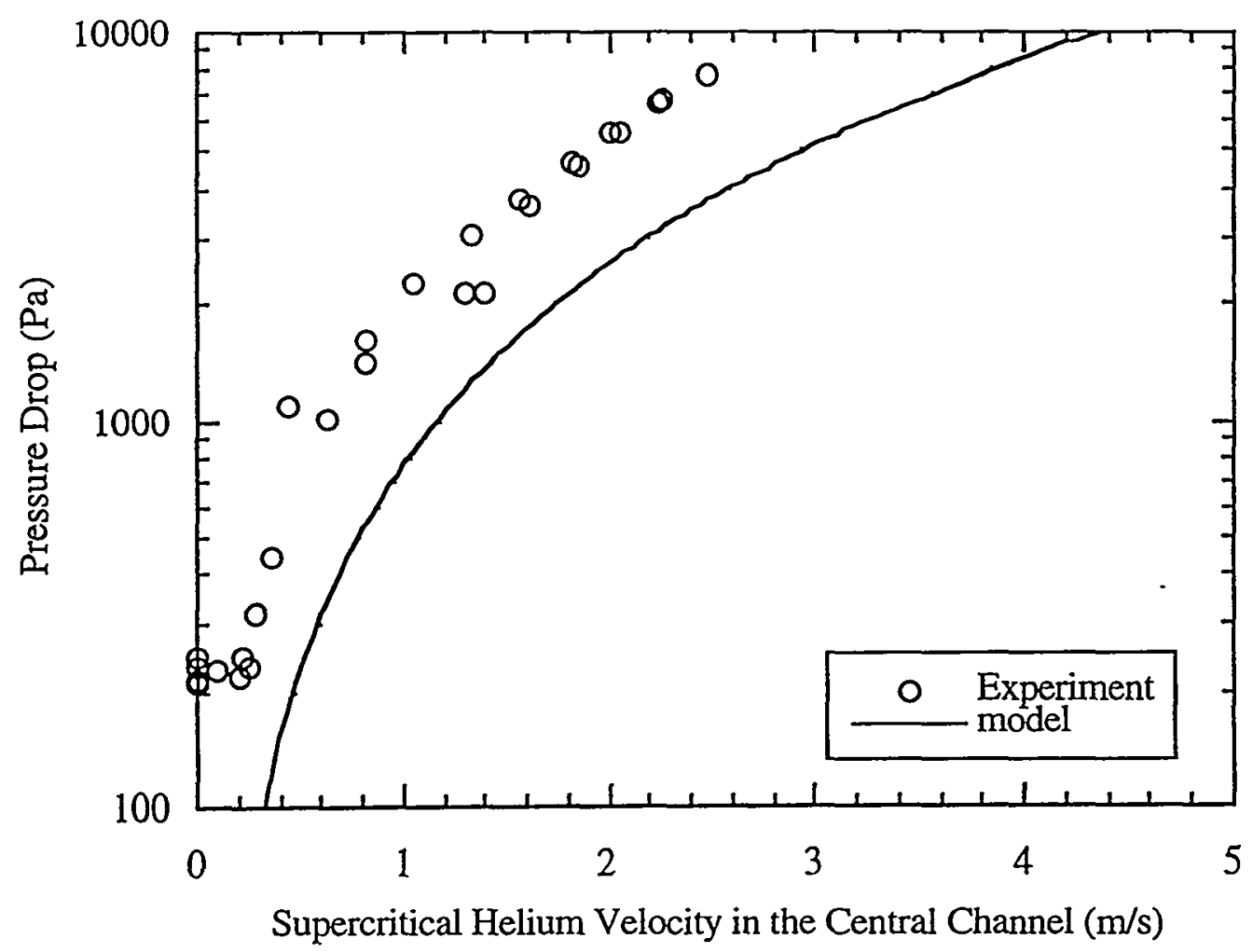

Figure 5.24. Comparison of the flow velocity in the central channel.

\subsubsection{Comparison of NET and CEA conductors}

Figure 5.25 shows friction factor variation as a function of Reynolds number for NET and CEA conductors. The flow area as well as cooled perimeter of each conductor was estimated by image analysis. First, the central channel of the CEA conductor proves to reduce the flow impedance of the CICC. The friction factor for the CEA conductor in high Reynolds number regime is approximately $40 \%$ lower than the NET conductor, which indicates the $\sim 40 \%$ of pressure drop reduction is accomplished by the hybrid cooling scheme. The reduction of flow impedance (or friction factor) of the conductor can improve: the frictional heating by 
supercritical helium, the maximum pressure rise of the helium during the quench of a superconducting magnet. It also greatly increases heat transfer between strands and the helium refrigeration system since the overall mass flow rate can be increased without significantly increasing the over all pressure drop. Further, the thermally induced flow velocity can be increased, which is mainly responsible for the excellent transient stability of the CICC type magnets.

Further study is necessary to understand the communication of supercritical helium between the strands region and in the central channel, which dominates the heat transfer mechanism of this new type of conductor. The ordinary parameters: hydraulic diameter and helium flow area, are not sufficient factors to characterize hydraulic behavior of the CICC. Based upon the hydraulic diameter of each conductor, the friction factor should show almost the same results for both conductors. Further, the NET conductor has larger flow area than that of the CEA conductor, however the friction factor does not reflect these differences. As discussed in previous chapter, the pressure drop depends on the geometry of the conductor but is not affected much by flow area or hydraulic diameter. Therefore, a new parameter is necessary for characterizing the hydraulic behavior of these new type CICCs which have a hybrid cooling channel. The pressure drop results however, shows that the hydraulic characteristics of the hybrid conductor can be described by those of the central channel: the six point star duct. This may be one way to characterize the new type Cable-in-Conduit Conductor. 


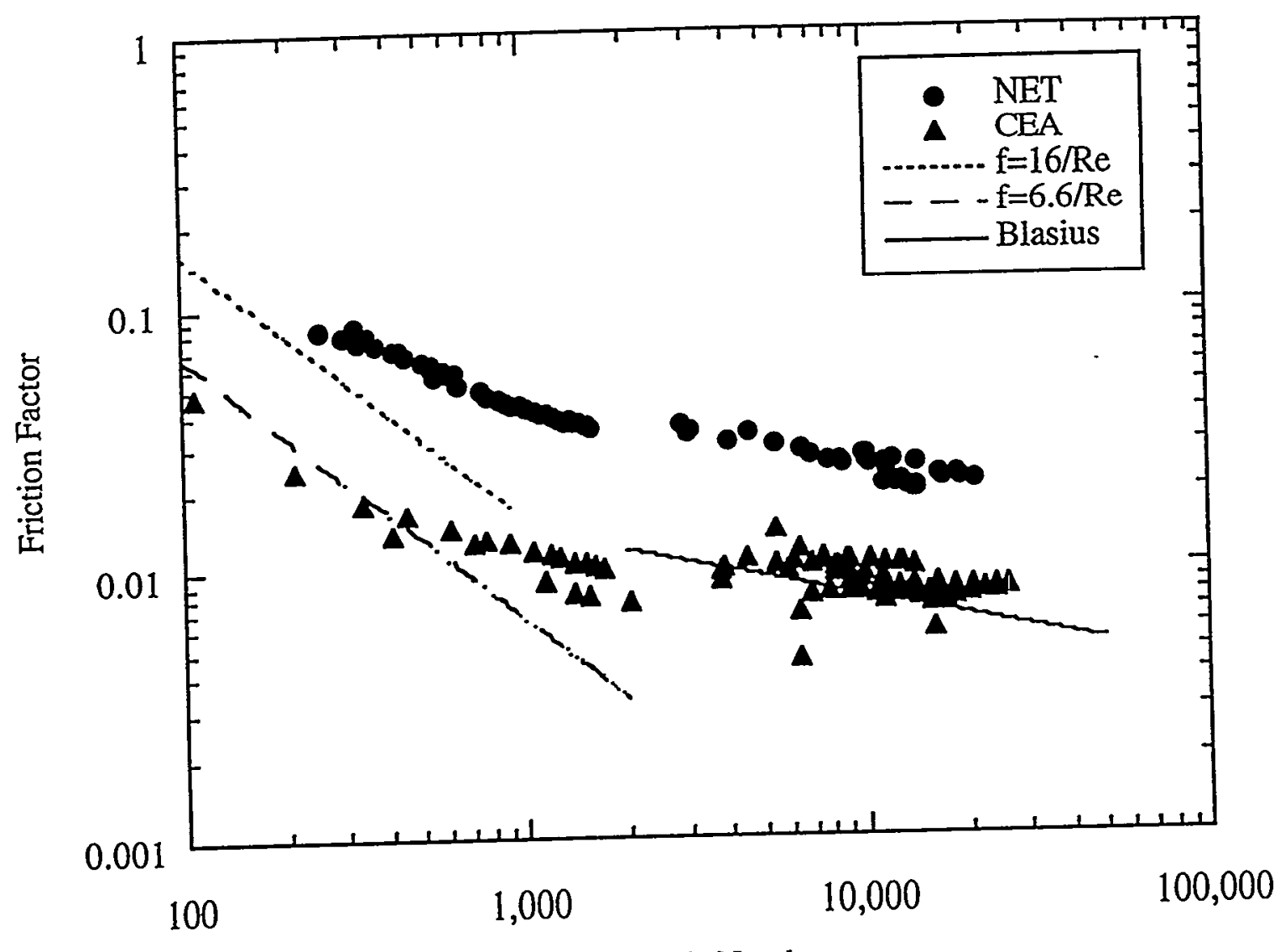

Reynolds Number

Figure 5.25. Friction factor vs. Reynolds number for NET and CEA conductor. 


\subsection{Inductive Heater Experiment}

\subsubsection{Calibration of the Inductive Heater}

The inductive heating method is the most practical way to deposit heat into CICCs, since the method does not affect an original configuration of the conductor. To accomplish this, it is essential to have an energy source which can generate a high current pulse with a time varying magnetic field. The capacitor bank system has been widely used as the energy source because of its flexibility and its efficiency. Figure 5.26 shows a basic electric circuit which generates the time varying magnetic field through the discharge of a capacitor bank. The secondary circuit shows a CICC, which simplifies the real circuit in the conductor. In general, the CICC consists of multistage strands which have different twist pitch in each stage, contact resistance of each strand as well as each stage are varies with location. The coupling loss of strands also varies at each stage. These factors strongly influence the eddy current calculation in the conductor. Therefore, experimental calibration of the inductive heater is required.
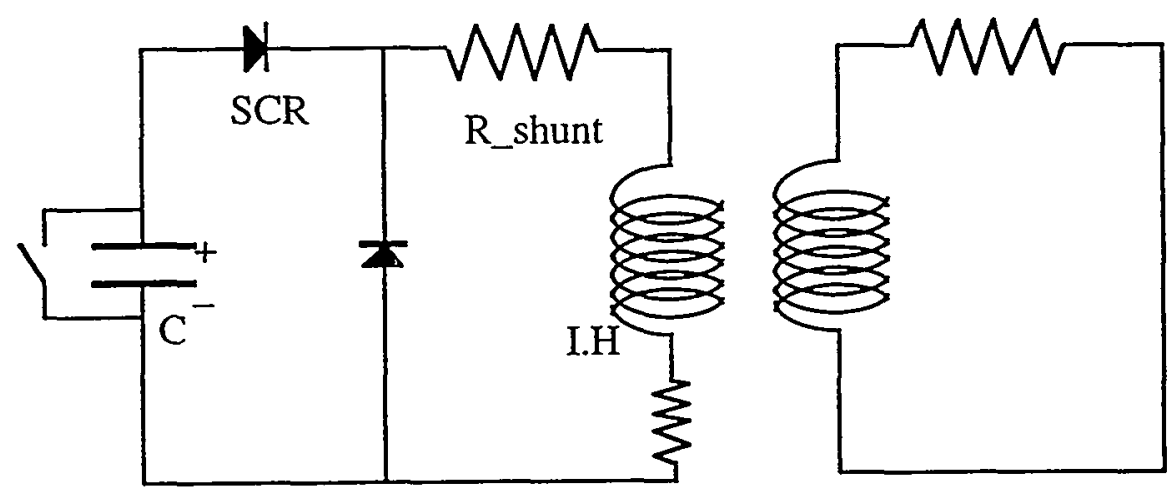

Figure 5.26. Equivalent electrical circuit for the inductive heating. 
The time varying field produced by the inductive heater induces eddy current in the strands. The heat is generated in the strands due to Joule heating, which is absorbed by the He II. The He II enthalpy change provides the information of energy deposit to the system. This calorimetric loss measurement was first employed by AC loss measurements of the SMES/CICC. 48

The calibration procedure is as follows. Liquid helium is first transferred to the helium bath and pumped to He II temperature: i.e. $\sim 1.8 \mathrm{~K}$. A press fit plug to the CICC, its controlling shaft located at room temperature, was used to isolate $\mathrm{He} I I$ in the conductor and the bath. Four Allen-Bradley thermometers monitor the He II temperature change in the central channel. The capacitor bank was charged up to a certain value and a computer sent an electric pulse to the SCR to discharge the capacitor bank. The He II temperature traces were stored in the computer. Figure 5.27 shows a typical temperature profile after the heat pulse. It took about $0.4 \mathrm{~s}$ to reach the isothermal condition.

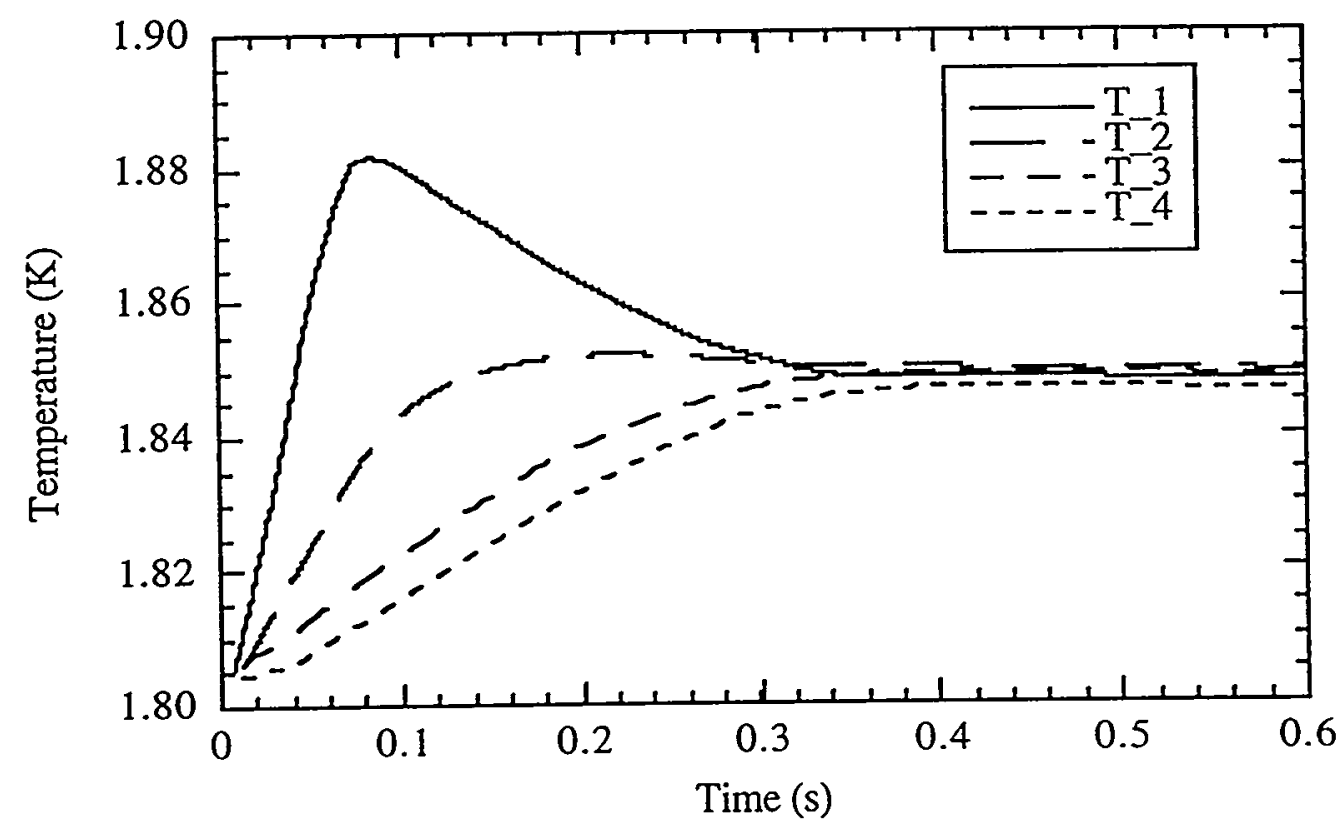

Figure 5.27. Typical calibration curve where stored energy was approximately $562 \mathrm{~J}$. 
Since the volume within the conductor is calculated based upon the image analysis, the He II enthalpy change gives the total energy deposit to the conductor as,

$$
\Delta h=\int_{T_{i}}^{T_{f}} \rho(T) C_{p}(T) d T
$$

where $\Delta h$ is specific enthalpy change $(\mathrm{J} / \mathrm{kg}), T_{i}$ and $T_{f}$ are initial and final temperature, respectively.

The inductive heater calibration results are shown in Figure 5.28. He II enthalpy change is a linear function of the stored energy in capacitor bank. The heater efficiency is very low; however, it is sufficient to deposit the energy starting from $\sim 250 \mathrm{~mJ} / \mathrm{cm}^{3}$ or more to the conductor which corresponds to the typical stability margin of the CICC.

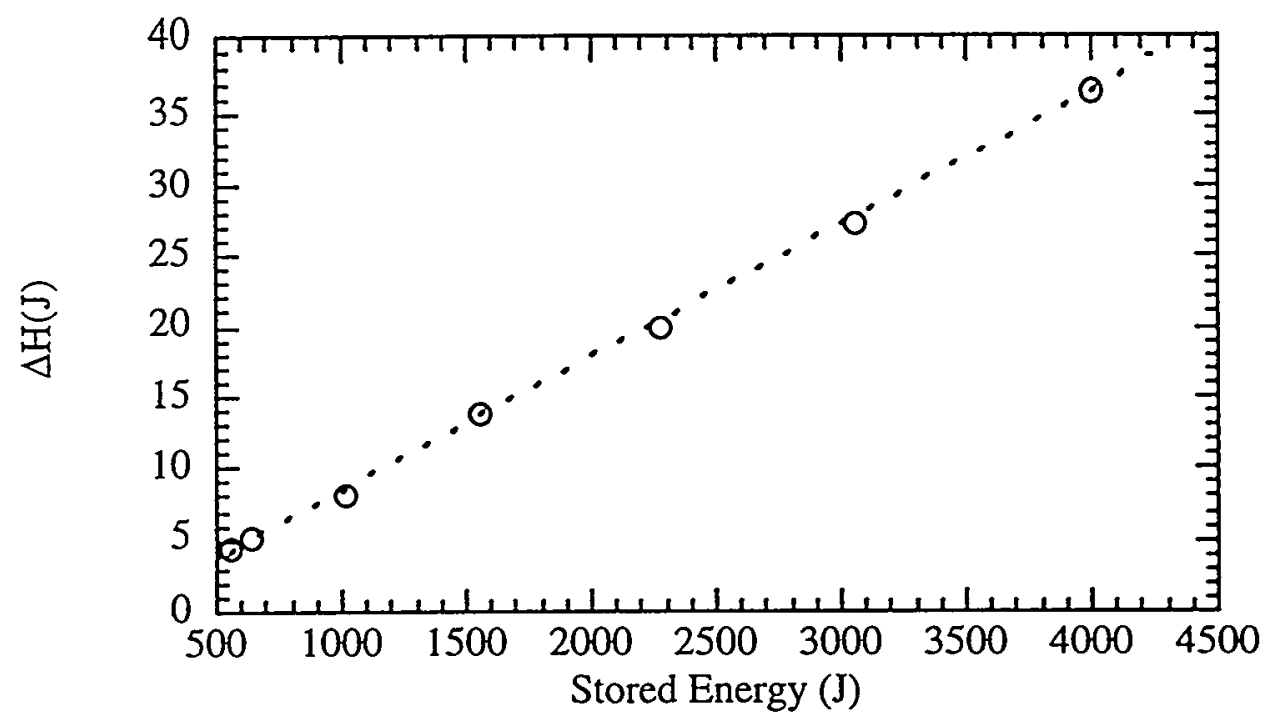

Figure 5.28. Inductive heater calibration results. 
Since the thermometers error is about $\pm 2 \mathrm{mK}$, this method provides a fairly accurate estimate of the energy input to the conductor. The error source is mainly the helium volume contained in the conductor, which is estimated to be around $10 \%$ or less.

Once the total energy input to the system is known, it is possible to calculate $Q(t)$. The simple way to estimate the $Q(t)$ is by eddy current generation in the strands, which is only dependent on the time varying magnetic field. The time constant of the CEA conductor is neglected for the calculation. The current through the inductive heater circuit is monitored by measuring a voltage across the shunt resistance. According to the Faraday's law,

$$
\oint E \cdot d l=\int \frac{\partial B}{\partial t} d S
$$

Eddy current generation can thus be expressed as,

$$
I(t)_{e d d y}=C \frac{E}{\rho_{e}}
$$

where $C$ is a numerical constant and $\rho_{e}$ is the electrical resistivity. The heat generated by the eddy current may thus be written as,

$$
Q(t)=I(t)_{\text {eddy }}^{2} R_{\text {strands }} \propto\left(\frac{d I(t)_{\text {magnet }}}{d t}\right)^{2}
$$

The integration of Equation (5.21) is equal to the He II enthalpy change. Note that $Q(t)$ is the total heat input to the conductor. Figure 5.29 shows the calculated $Q(t)$ from $I(t)_{\text {magnet }}$ and He II enthalpy change as function of time. 


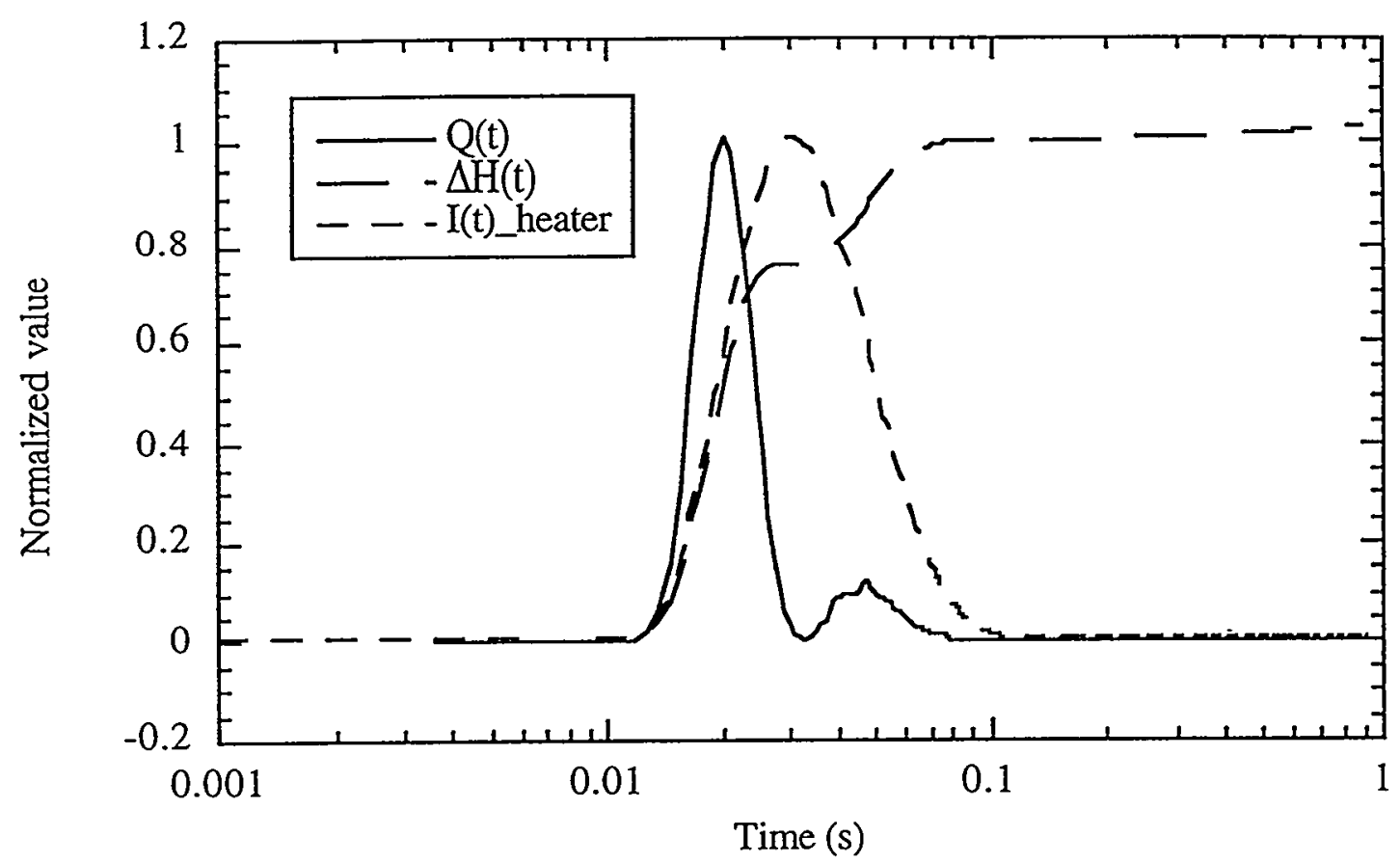

Figure 5.29. Estimated $\mathrm{Q}(\mathrm{t})$ and $\mathrm{H}(\mathrm{t})$ from measured voltage across the resistance.

\subsubsection{Thermally-induced Flow Experiment.}

Thermally-induced flow and pressure rise of the low temperature helium in the CEA conductor have been studied. The transient increase of both total mass flow rate and helium velocity in the central channel were measured as function of energy input and imposed mass flow rate. The applied heat input to the conductor varied from $200 \mathrm{~kJ} / \mathrm{m}^{3}$ to $\sim 1600 \mathrm{~kJ} / \mathrm{m}^{3}$, while the Reynolds number was adjusted between zero and about 8000 . Supercritical helium temperature was approximately $4.3 \mathrm{~K}$ and pressure was about $0.3 \mathrm{MPa}$. Saturated $\mathrm{He} \mathrm{II}$ was also used in the no flow condition: the He II temperature was about $1.8 \mathrm{~K}$ and pressure was about $1700 \mathrm{~Pa}$. This section presents the results and analysis of the thermally induced flow and pressure rise experiment. 


\subsubsection{Stagnant Supercritical Helium and He II}

Figures 5.30 (a), (b), (c) and (d) show the induced flow for different heat inputs to the conductor with supercritical helium. Both induced total mass flow rate and the helium velocity in the central channel are plotted in the same graph for a comparison. The time to reach the initial condition $\dot{m}=0$ was about one second for all heat inputs. As expected, increasing the heat input induces more transient flow to the conductor; however, in some cases the velocity profile is distorted as the peak in the central channel velocity occurred later than that in the total mass flow rate, see Figures 5.30 (c) and (d). This effect is mainly caused by the pressure wave propagation through the conductor. As shown in Figures 5.31 (a) and (b), the pressure wave propagates at the speed of sound to both ends of the conductor. A transient static pressure rise for shot \# 17 was twice as high as that for shot \#16. Therefore, the velocity profile in shot \#17 shows several noisy peaks from $0.1 \mathrm{~s}$ to $0.5 \mathrm{~s}$. Neither shot \#14 or \#15 showed significant pressure increase due to rather small heat inputs.

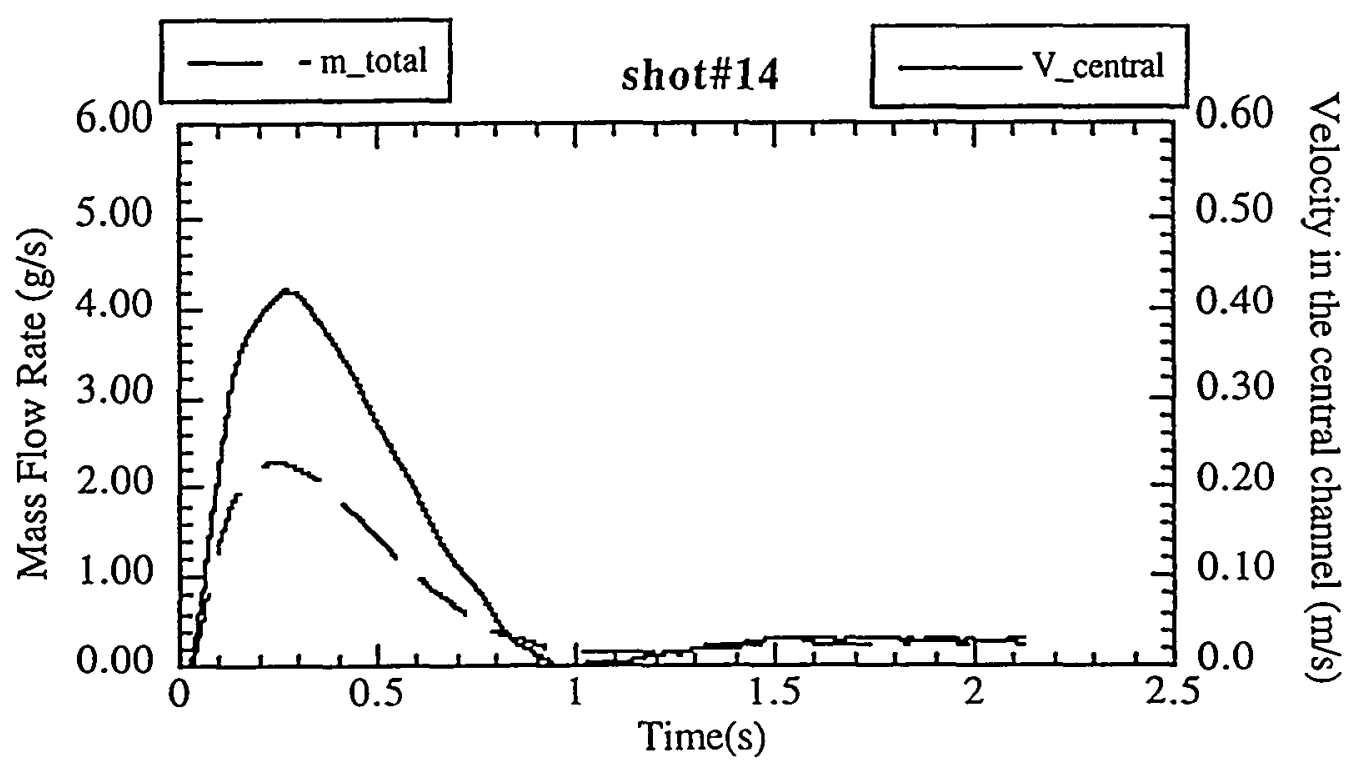

Figure 5.30a. Mass flow rate and v_central vs. time. 


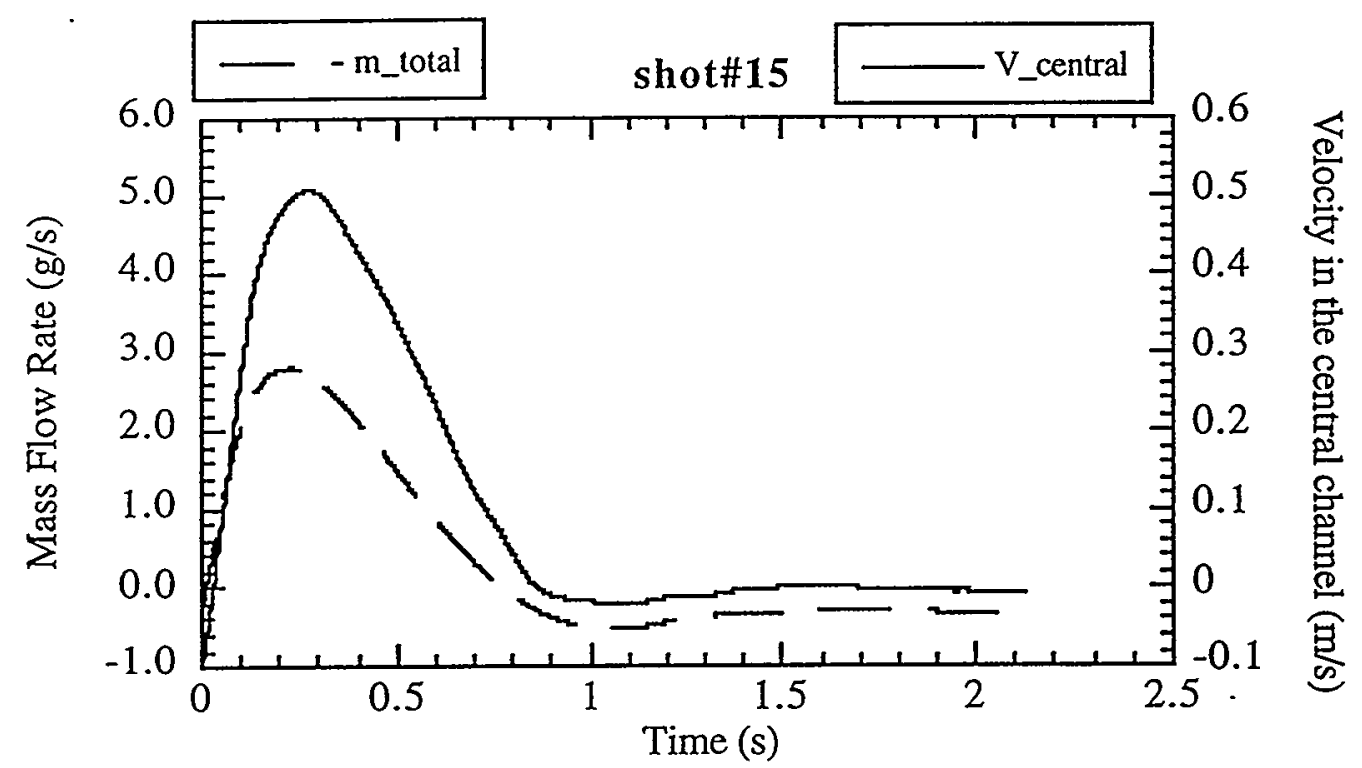

Figure 5.30b. Mass flow rate and v_central vs. time.

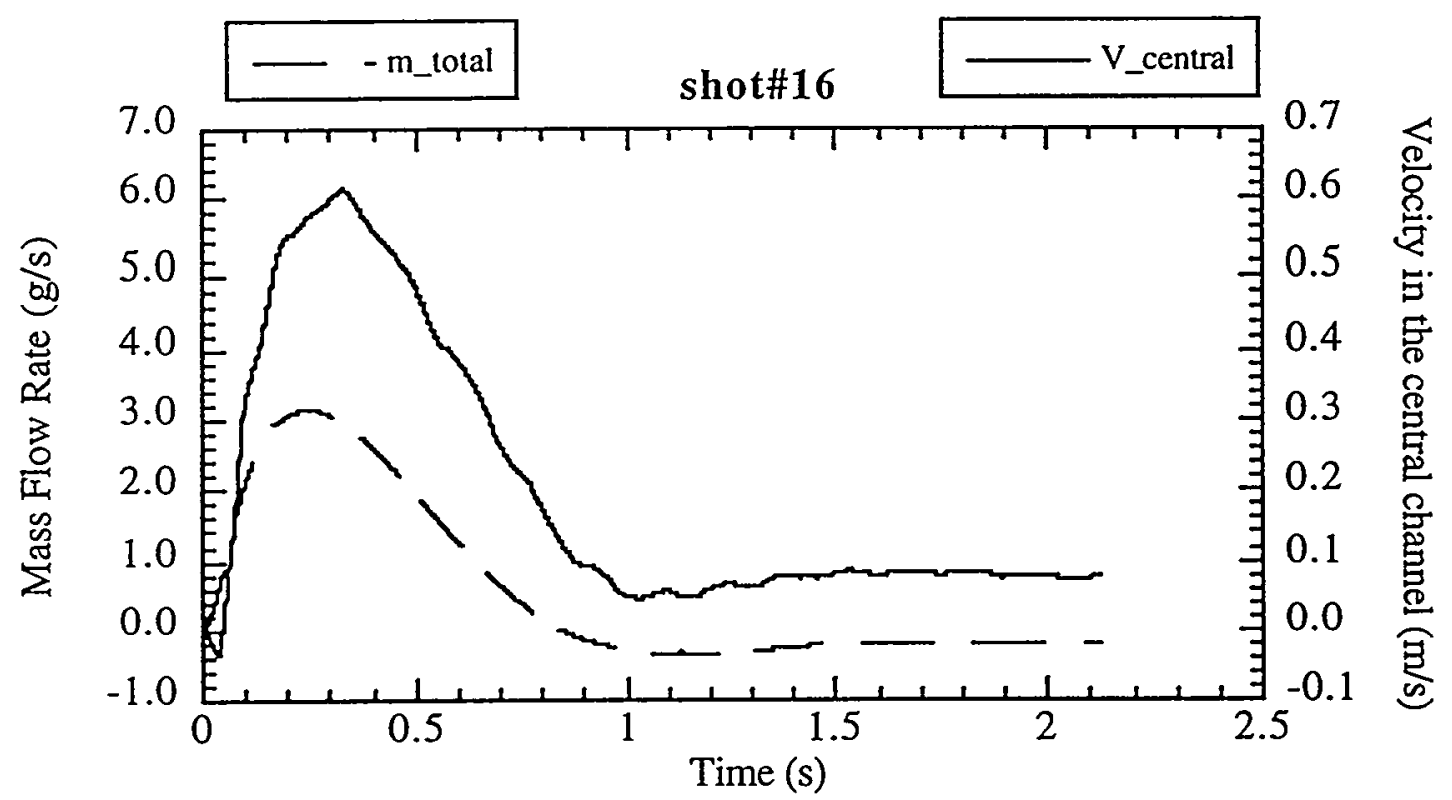

Figure 5.30c. Mass flow and v_central vs. time. 


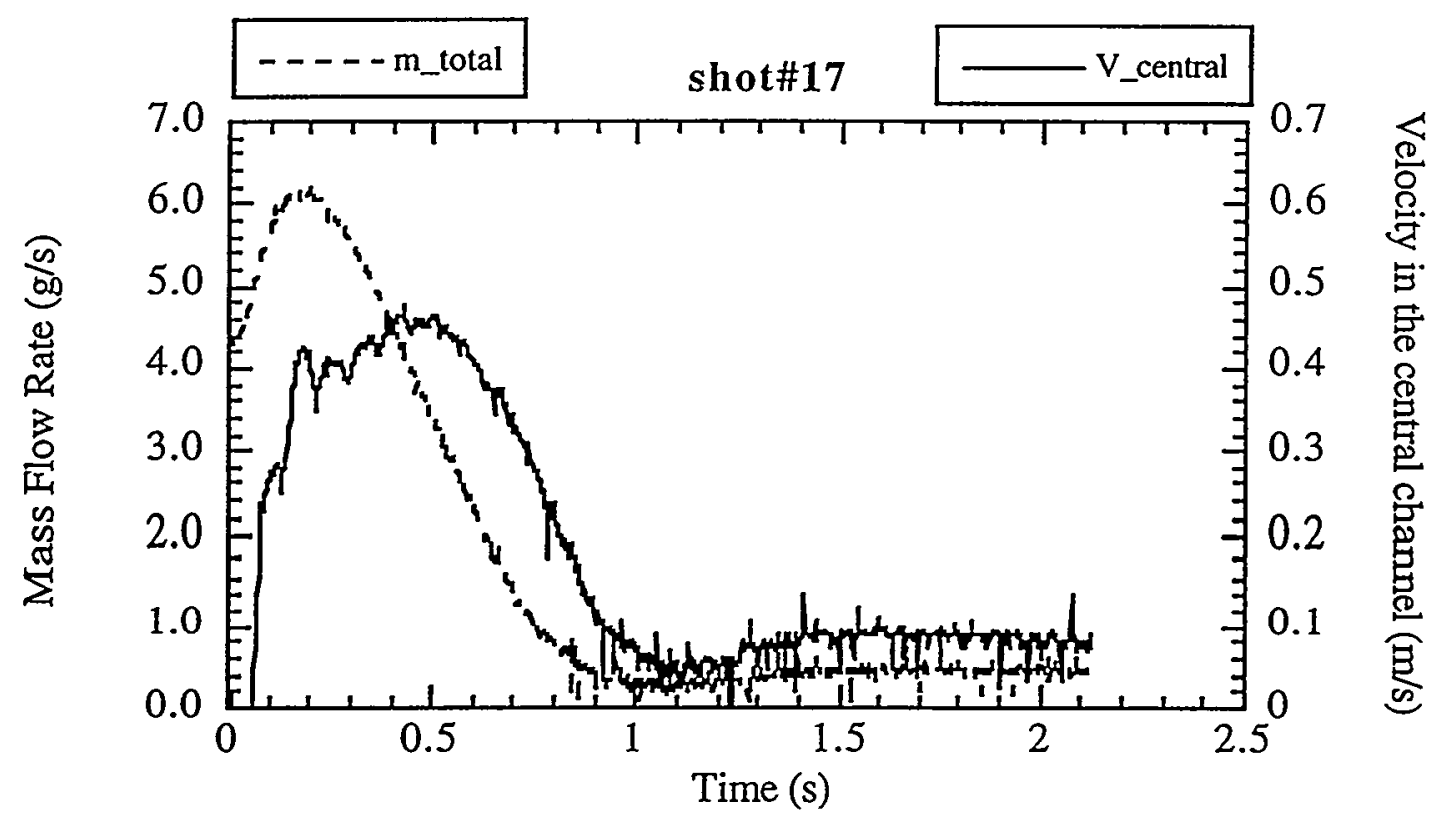

Figure 5.30d. Mass flow and v_central vs. time.

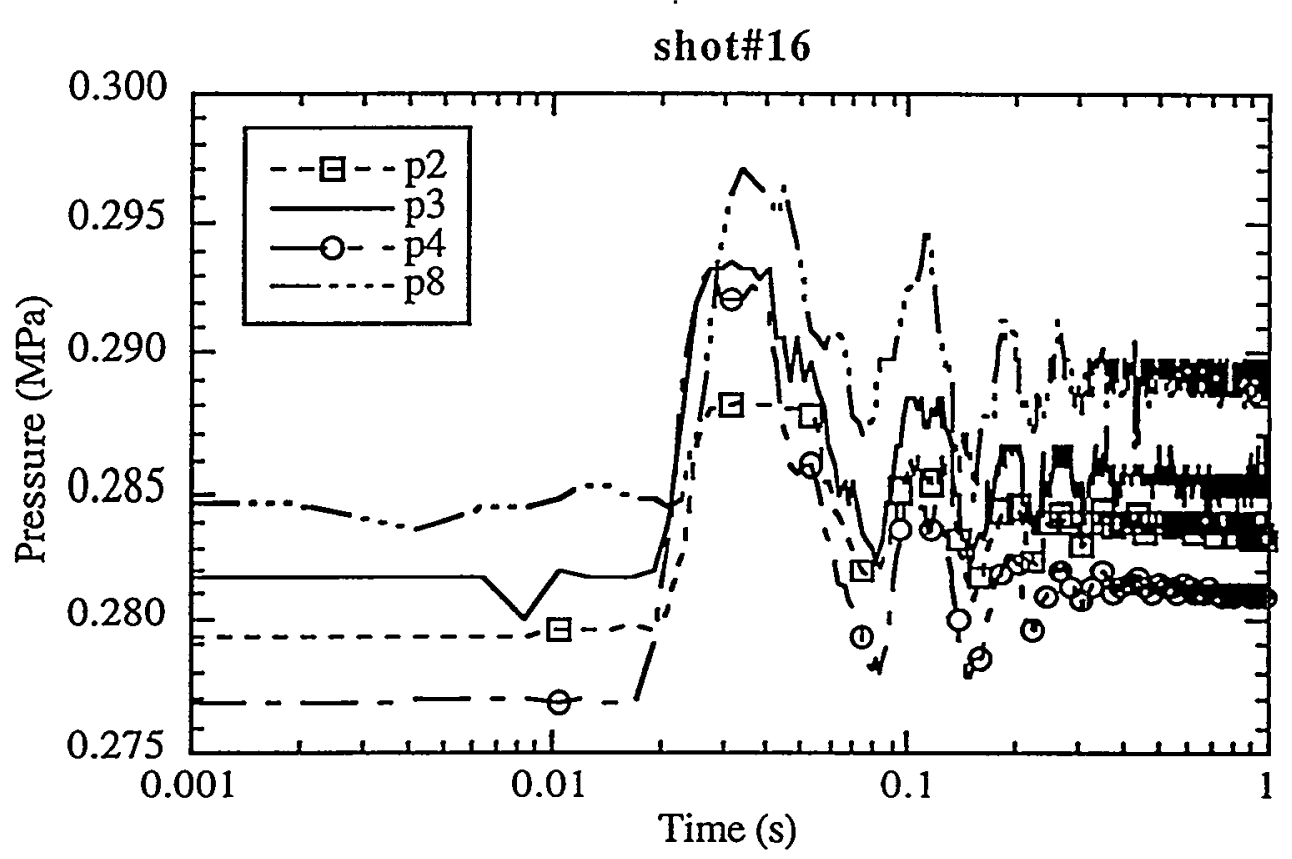

Figure 5.31a. Static pressure vs. time for shot \#16 


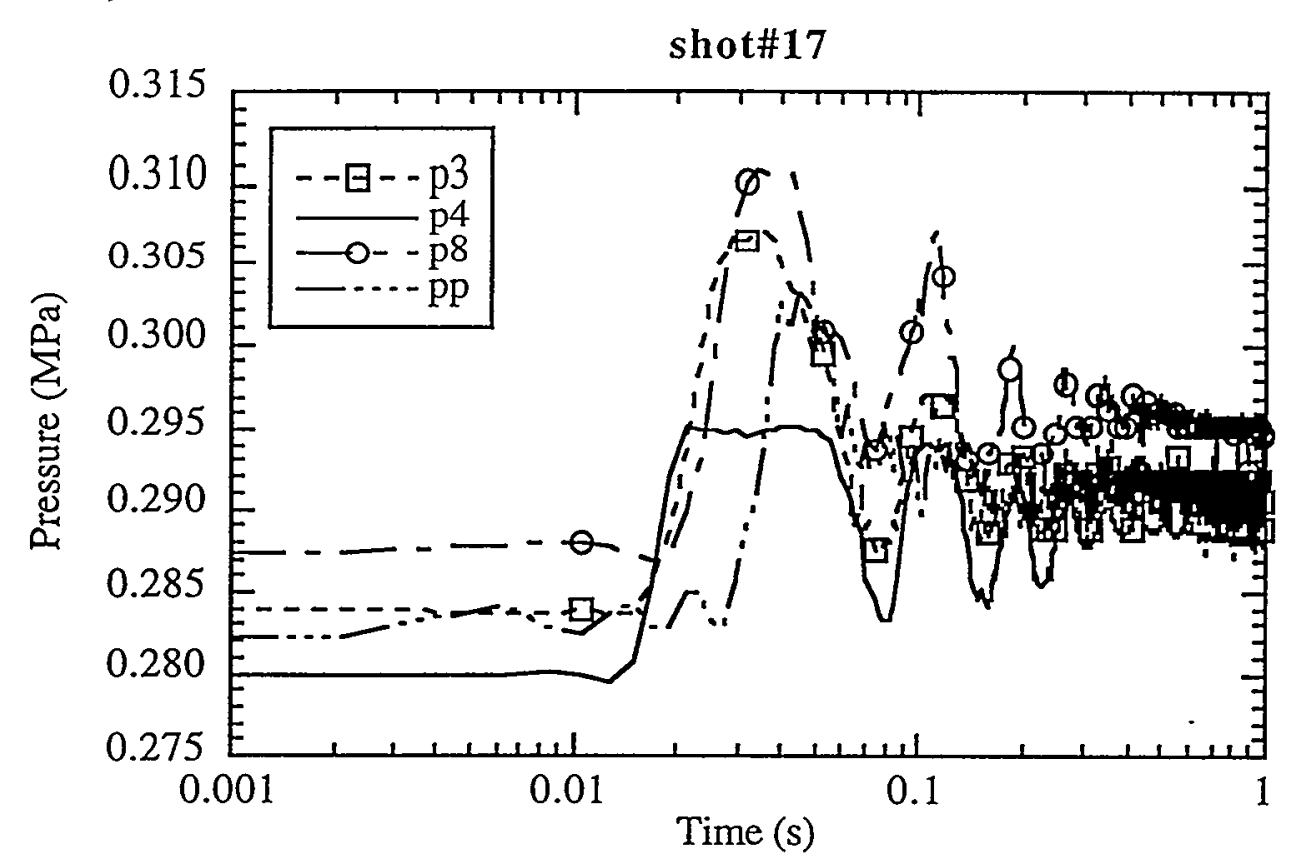

Figure 31b. Static pressure vs. time for shot \#17.

The induced mass flow rate in the central channel is calculated by,

$$
\dot{m}(t)=\bar{\rho} A_{\text {cenral }} v_{\text {induced }}(t)
$$

where $\bar{\rho}$ is unperturbed helium density. The calculation results are compared with the induced total mass flow rate as shown in Figure 5.32 (a), (b), (c), for shots \#14, 15 and 16. The calculated mass flow rate in the central channel is about $50 \%$ larger than the total mass flow rate. This may suggest that the flow is not fully established across the cross section of the conductor. Thus, the measured velocity in the central channel is the maximum velocity. In other words, the helium within the bundle region is assumed to be stationary. Although the pulse heat input to the conductor longitudinally expands the supercritical helium, the helium in the bundle regime loses its kinetic energy faster than in the central channel because 
of larger flow impedance and the heat transfer to the low temperature strands. Further, the helium flow area in the bundle region is approximately five times larger than in the central channel. Figure 5.33 shows the warm helium behavior in the conductor based on this assumption.

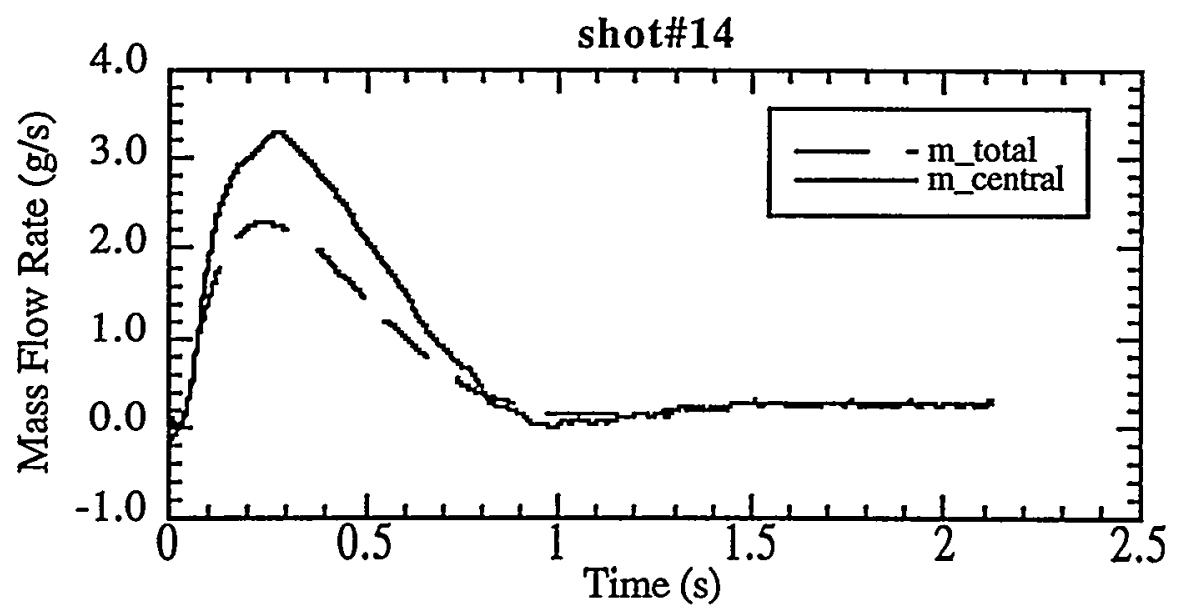

Figure 5.32a. Mass flow rate vs. time for shot \#14.

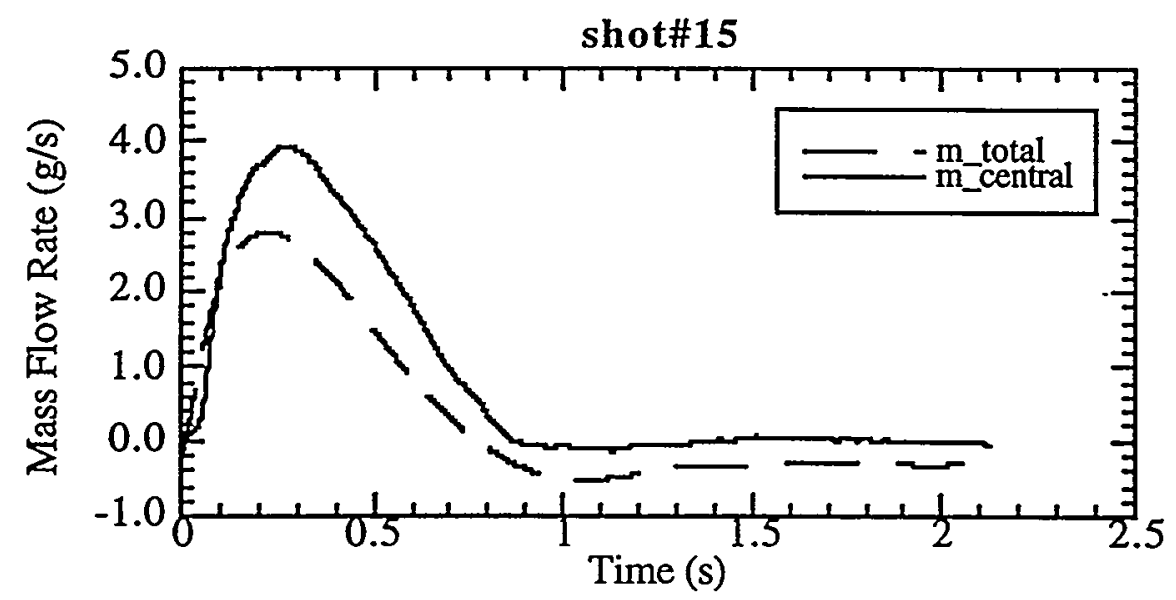

Figure 5.32b. Mass flow rate vs. time for shot \#15 


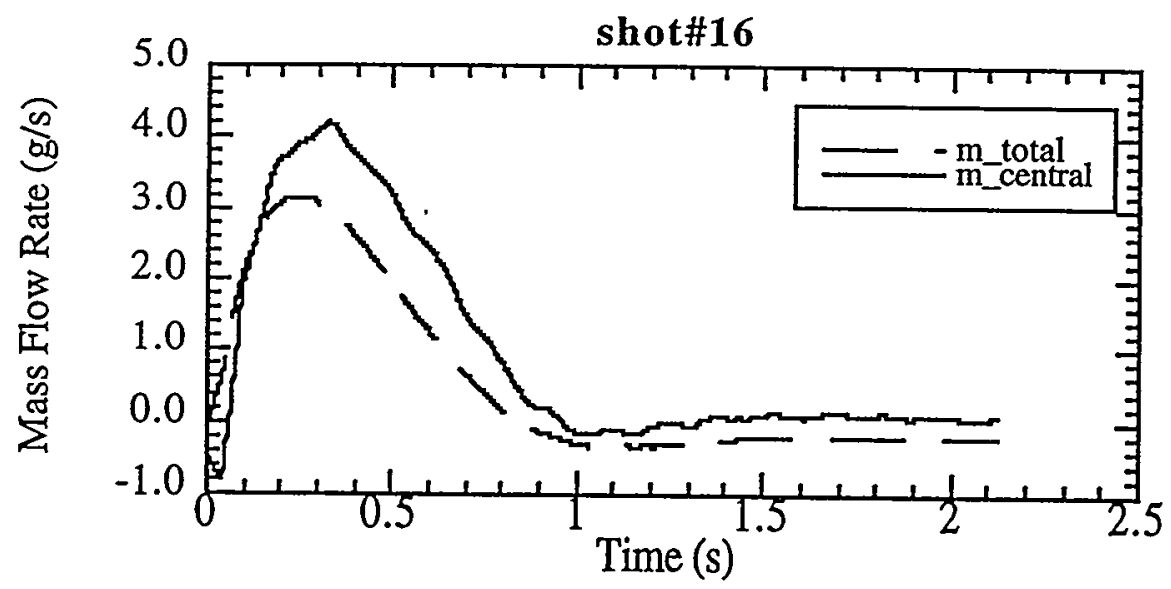

Figure 5.32c. Mass flow rate vs. time for shot \#16.
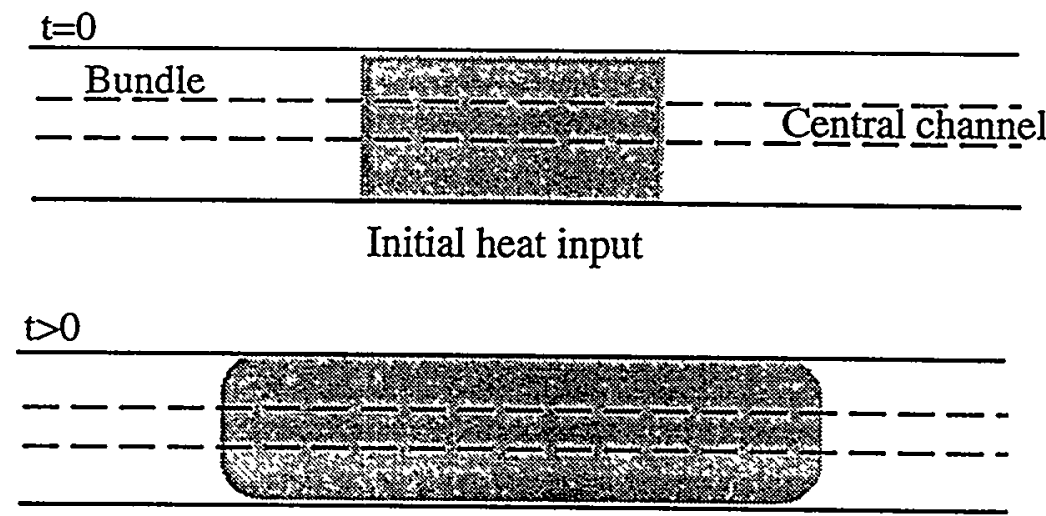

Diffusion process

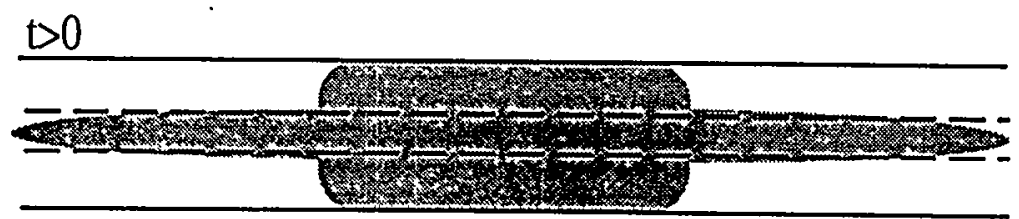

Hydraulic process

Figure 5.33. Thermal hydraulic behavior of supercritical helium in the conductor. (A shaded region shows the warm helium) 
Thermally induced flow of He II was also measured as a function of heat input. Figures 5.34(a) and (b) show the typical measured mass flow rate in the central channel and total mass flow rate. Unlike the supercritical helium measurements, the mass flow rate in the central channel is approximately $80 \%$ of the total. The flow velocity increases with increasing energy input. The mechanism of the induced flow in the He II is different from that in the supercritical helium since the He II vaporizes by absorbing the heat from the strands. Consequently, the vapor pushes the liquid to both ends. Further, the lower viscosity of $\mathrm{He} \mathrm{II}$ results in the smooth induced flow in the conductor. However, the mass flow rate in the central channel shows a higher value than a steady state. This may primarily be caused by the non steady state nature of the induced flow. Figure 5.35 shows the induced flow velocity change with energy input.

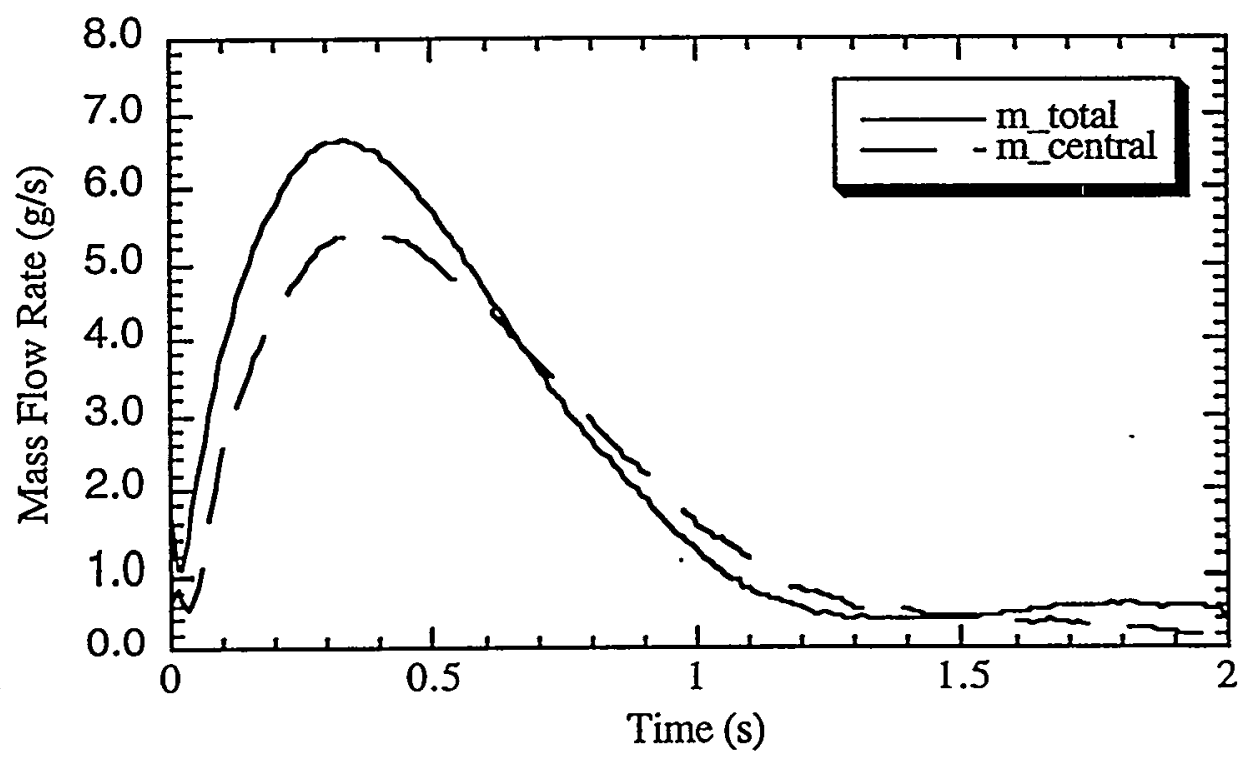

Figure 5.34a. Induced mass flow rate with heat input $36 \mathrm{~J}$ 


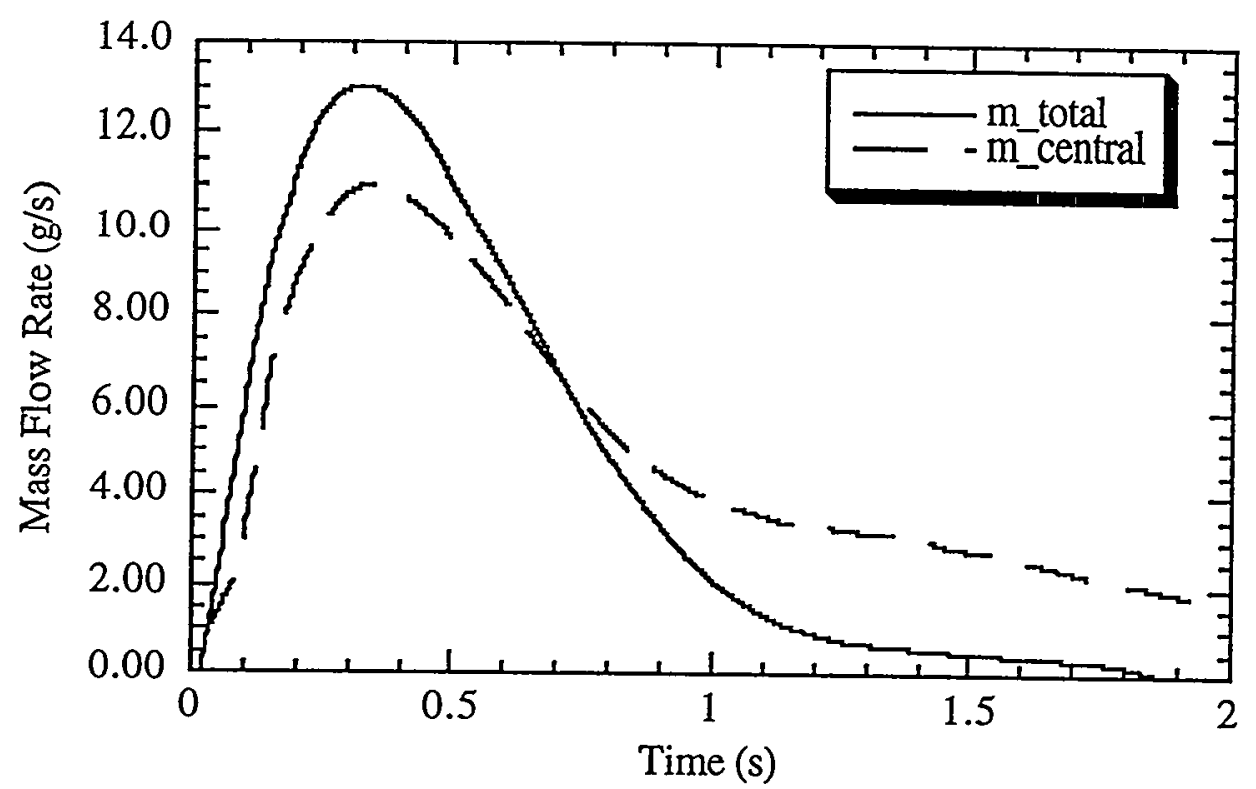

Figure 5.34b. Induced mass flow rate with heat input $58 \mathrm{~J}$.

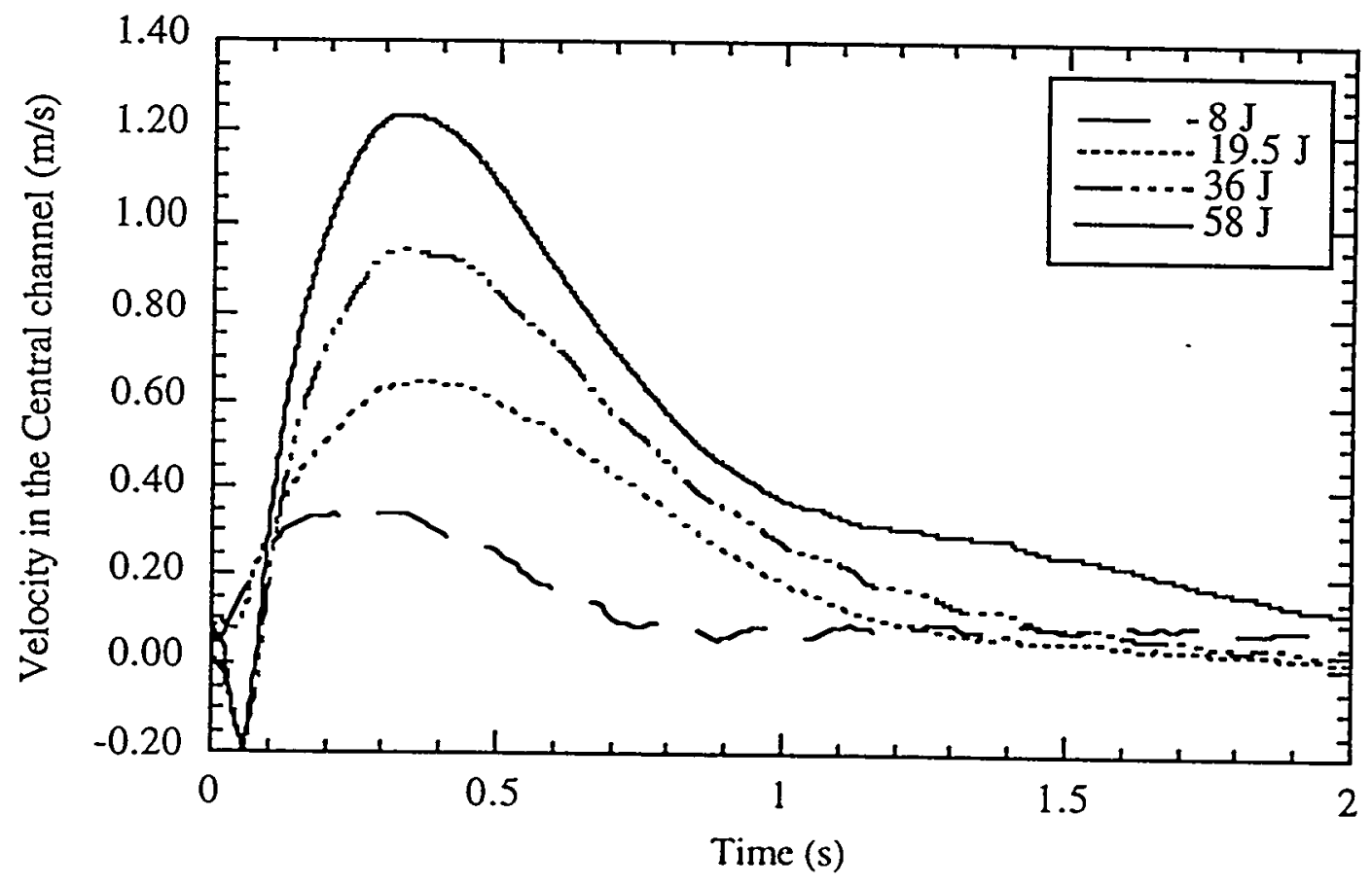

Figure 5.35. Induced flow velocity in He II with different energy input. 
The experimental results are compared with a Dresner's ${ }^{26}$ thermally induced flow formula,

$$
u_{\text {induced }} \sim \frac{\dot{q} P_{\text {cooled }} l}{A_{\text {flow }}} \frac{\beta}{\rho C_{p}}=\frac{\Delta H \beta}{A_{\text {flow }} \rho C_{p} \Delta t}
$$

where $\Delta H$ is a total heat input and $\Delta t$ is pulse duration. Figure 5.36 shows the calculation results compared with experiment.

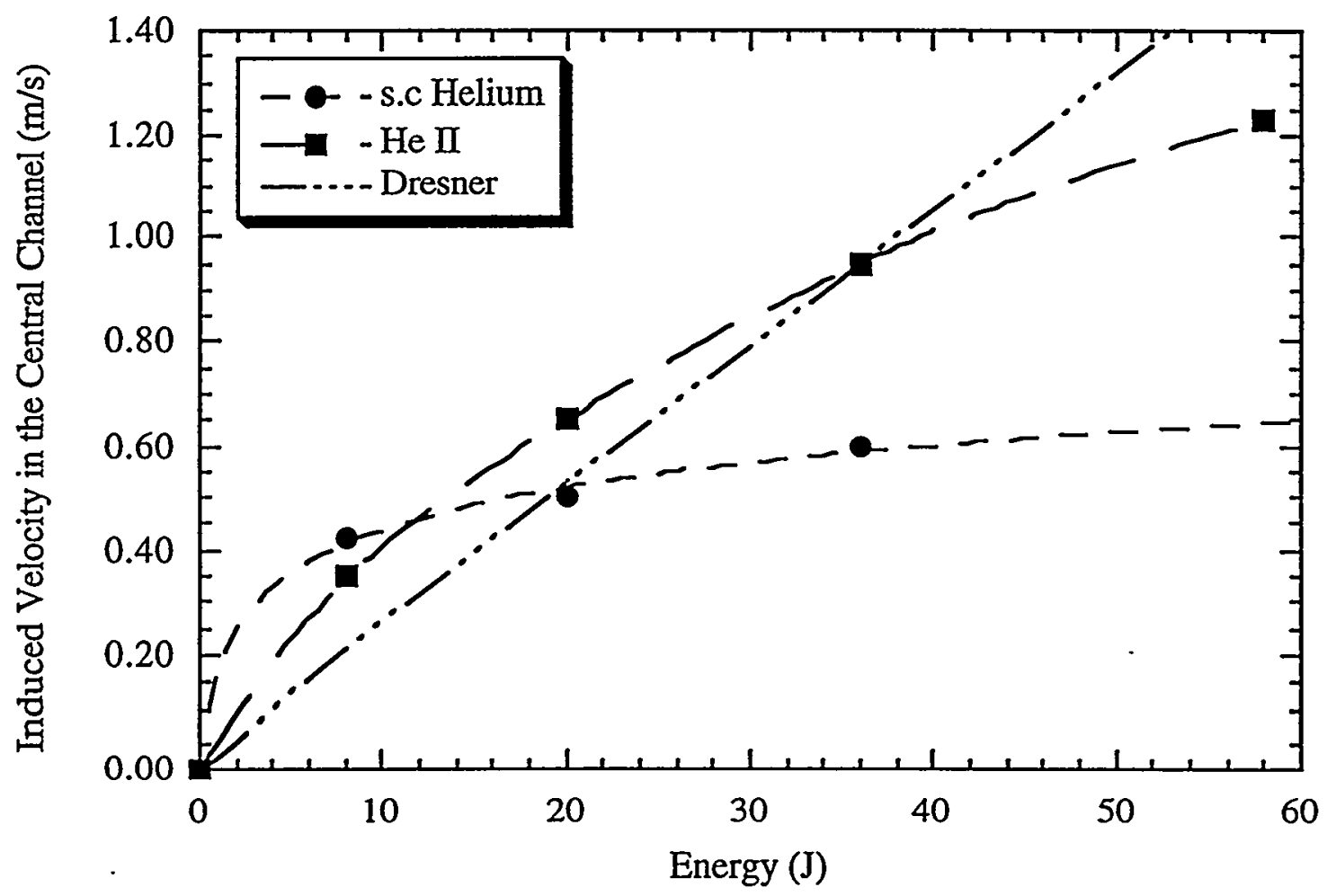

Figure 5.36. Comparison of thermally induced flow velocity with theory.

Dresner assumed a linear dependence of the induced flow, constant friction factor and thermophysical properties. In reality, the friction factor and unperturbed supercritical helium affect the flow condition so that the velocity increase becomes smaller as heat input 
increases. In supercritical helium, the thermally induced flow velocity saturates in the high energy input region. The induced flow in He II follows the same tendency as supercritical helium.

\subsubsection{Forced Flow Supercritical Helium}

Figures 5.37 (a), (b), (c) and Figures 5.38 (a), (b), (c) show the experimental results with imposed mass flow rates $\dot{m}_{i} \approx 10.5 \mathrm{~g} / \mathrm{s}, R e \approx 5100$ and $\dot{m}_{i} \approx 14.5, R e \approx 7050$, respectively. For each flow condition, energy inputs of $8 \mathrm{~J}, 19.5 \mathrm{~J}$ and $36.5 \mathrm{~J}$ were applied, . The total induced mass flow rates were not significantly changed with various energy inputs. However, the induced flow velocity in the central channel becomes steeper with increasing the heat inputs. These data suggest the thermally induced flow in the conductor is primarily generated in the central channel and that the transient disturbance introduced in the conductor is carried away by the helium in the central channel. This is mainly caused by the difference of hydraulic characteristics between the central channel and the bundle region.

The effects of heat input on the central channel velocity are seen in Figures 5.39 (a), (b) for different imposed flows. Although the velocity profile changes with increasing heat input, the induced flow velocity is suppressed because of the increased heat transfer coefficient with increasing helium velocity. Figure 5.40 shows the fractional increase of the induced flow velocity in the central channel as function of energy input with different imposed flow rates. In the figure, the number beside the legend corresponds to the initial Reynolds number. Reduction of the induced flow velocity is clearly associated with increasing the imposed mass flow rate. 


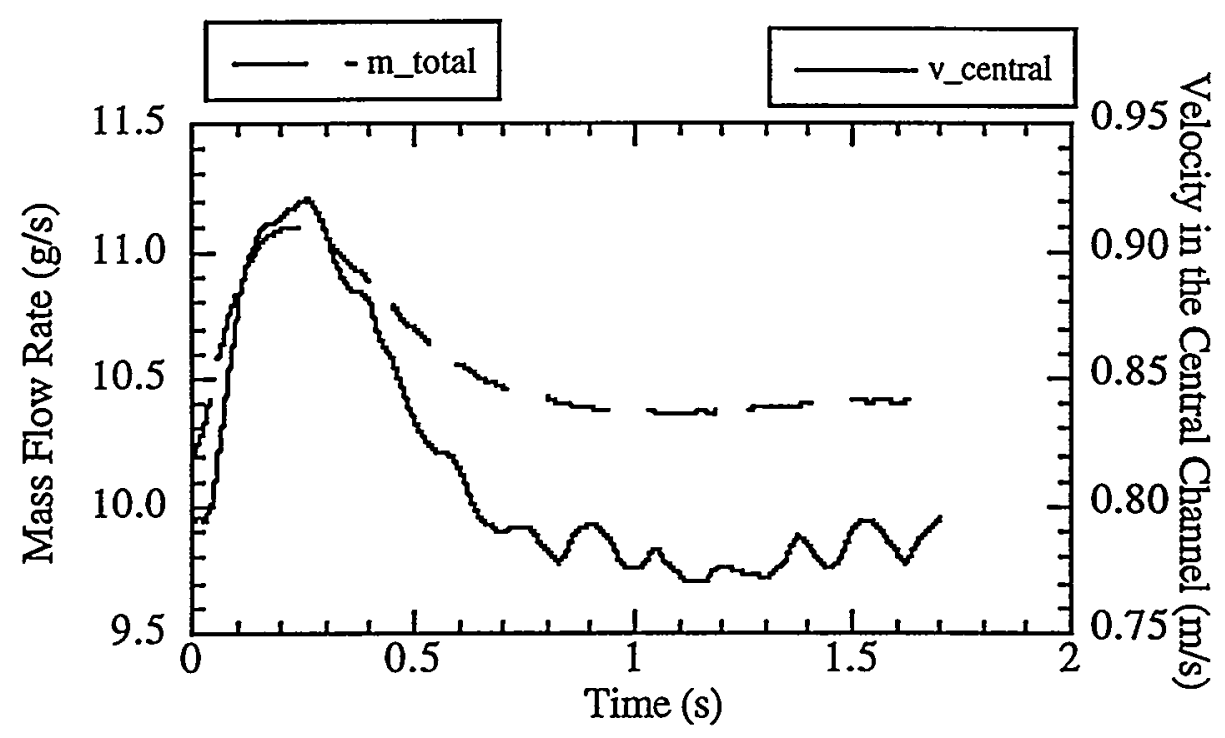

Figure 5.37a. Induced total mass flow rate and velocity in the central channel. $(8 \mathrm{~J})$ $\operatorname{Re}=5100$

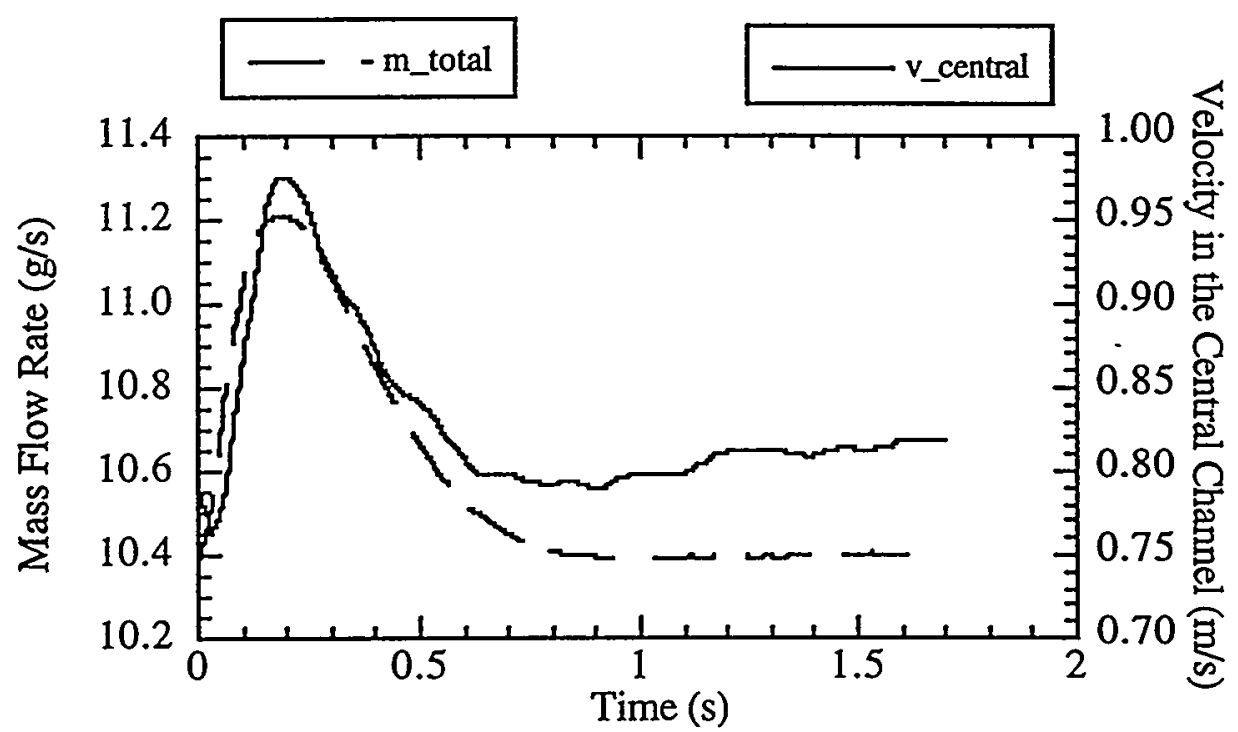

Figure 5.37b. Induced total mass flow rate and velocity in the central channel. (19.5 J) $\mathrm{Re}=5100$ 


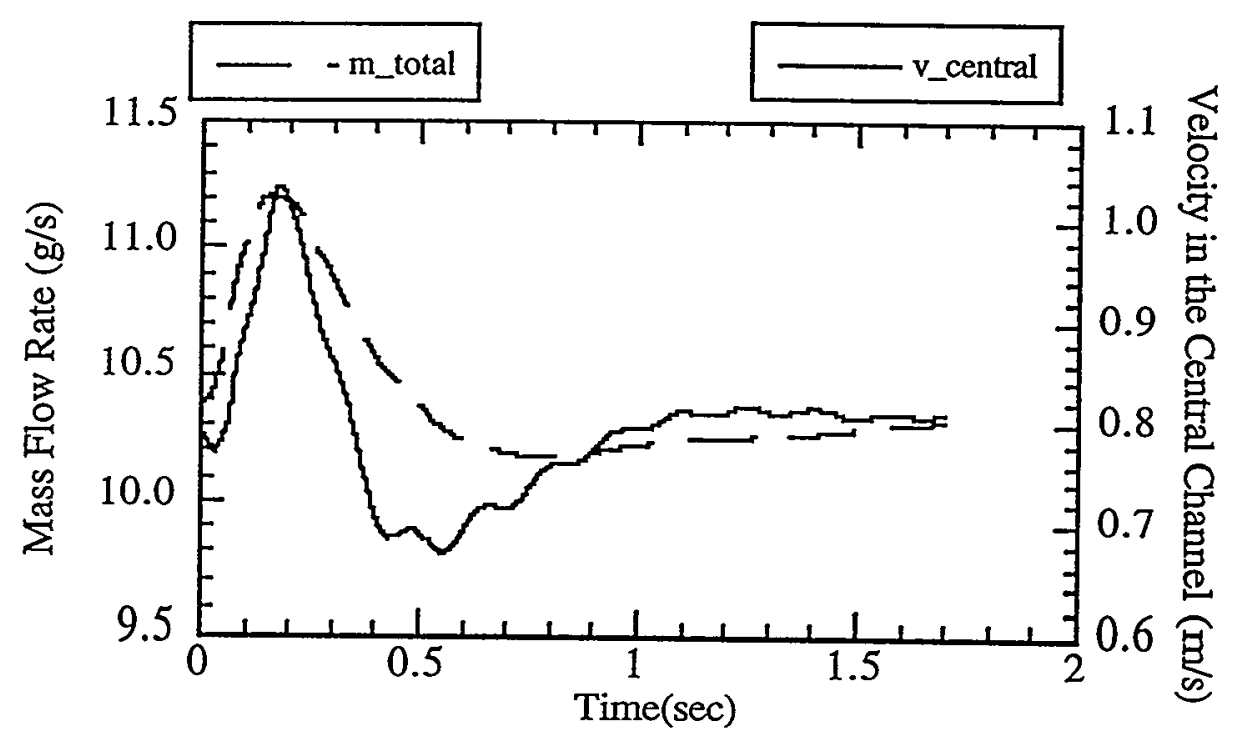

Figure 5.37c. Induced total mass flow rate and velocity in the central channel. $(36.5 \mathrm{~J})$ $\mathrm{Re}=5100$

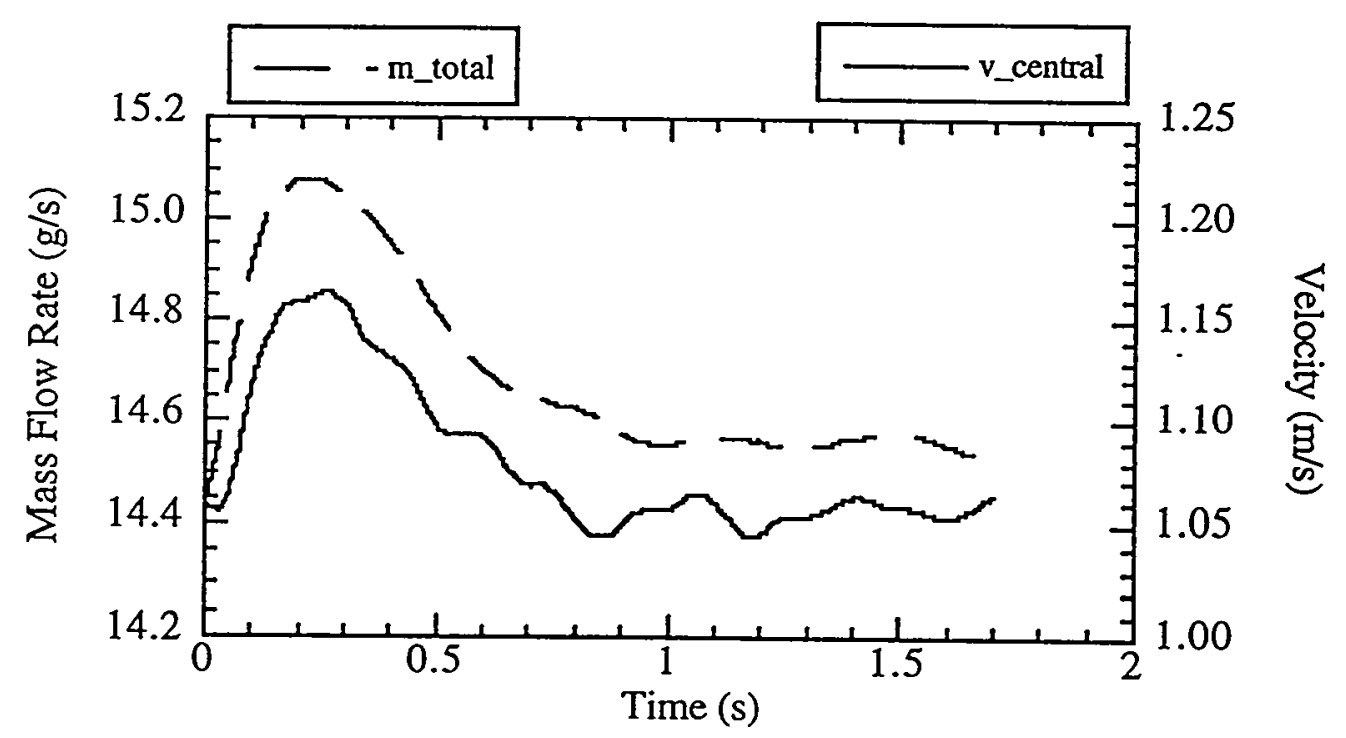

Figure 3.38a. Induced total mass flow rate and velocity in the central channel (8 J). $\mathrm{Re}=7050$ 


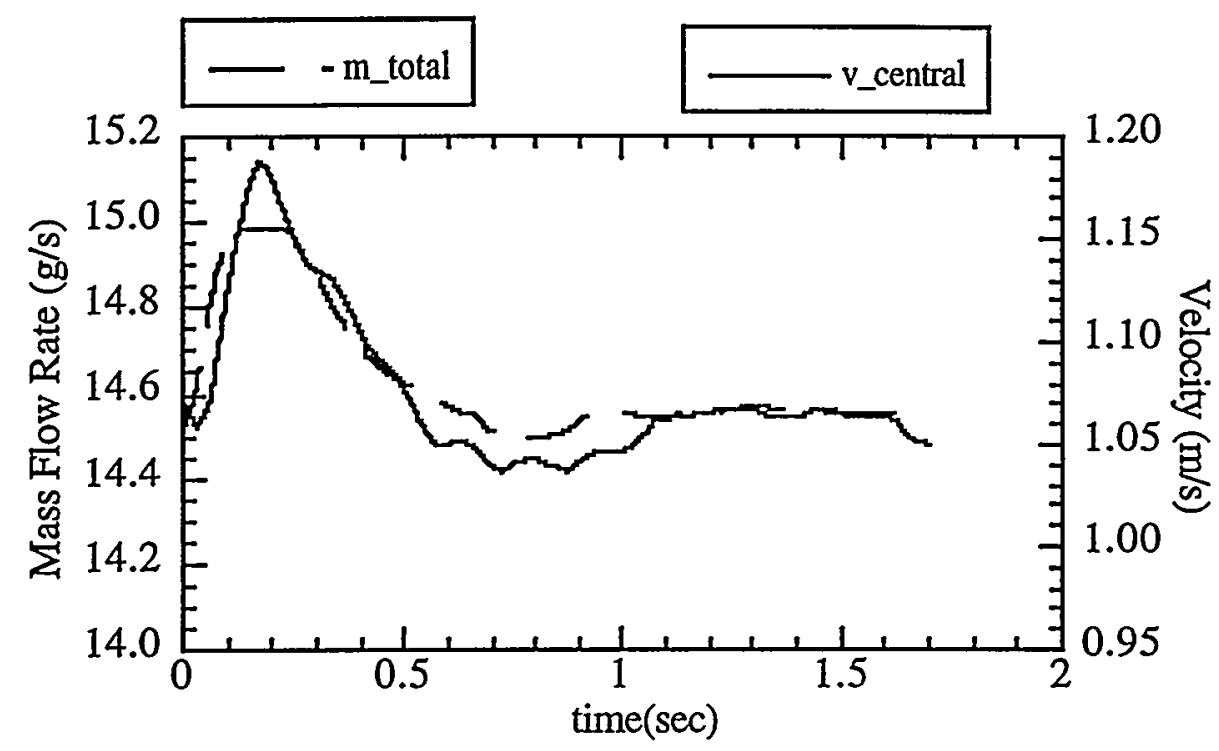

Figure 3.38b. Induced total mass flow rate and velocity in the central channel $(19.5 \mathfrak{J})$. $\operatorname{Re}=7050$

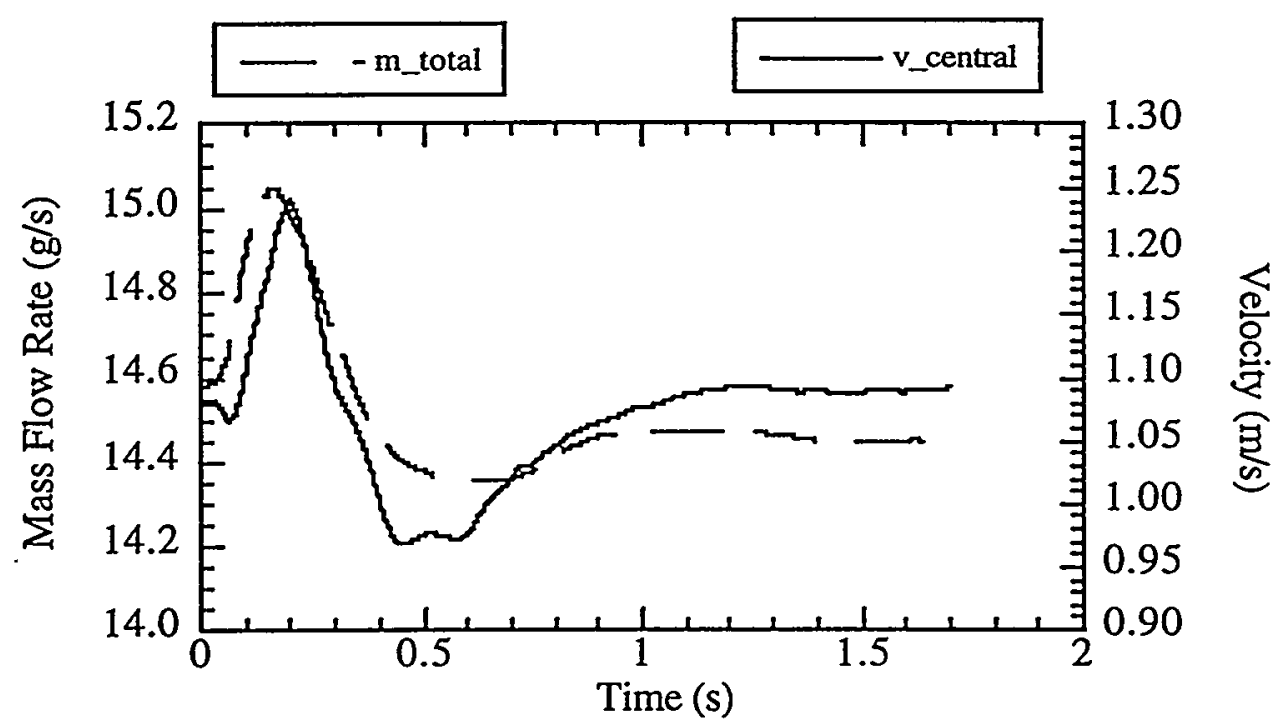

Figure 3.38c. Induced total mass flow rate and velocity in the central channel ( $36.5 \mathrm{~J})$.

$$
\mathrm{Re}=7050
$$




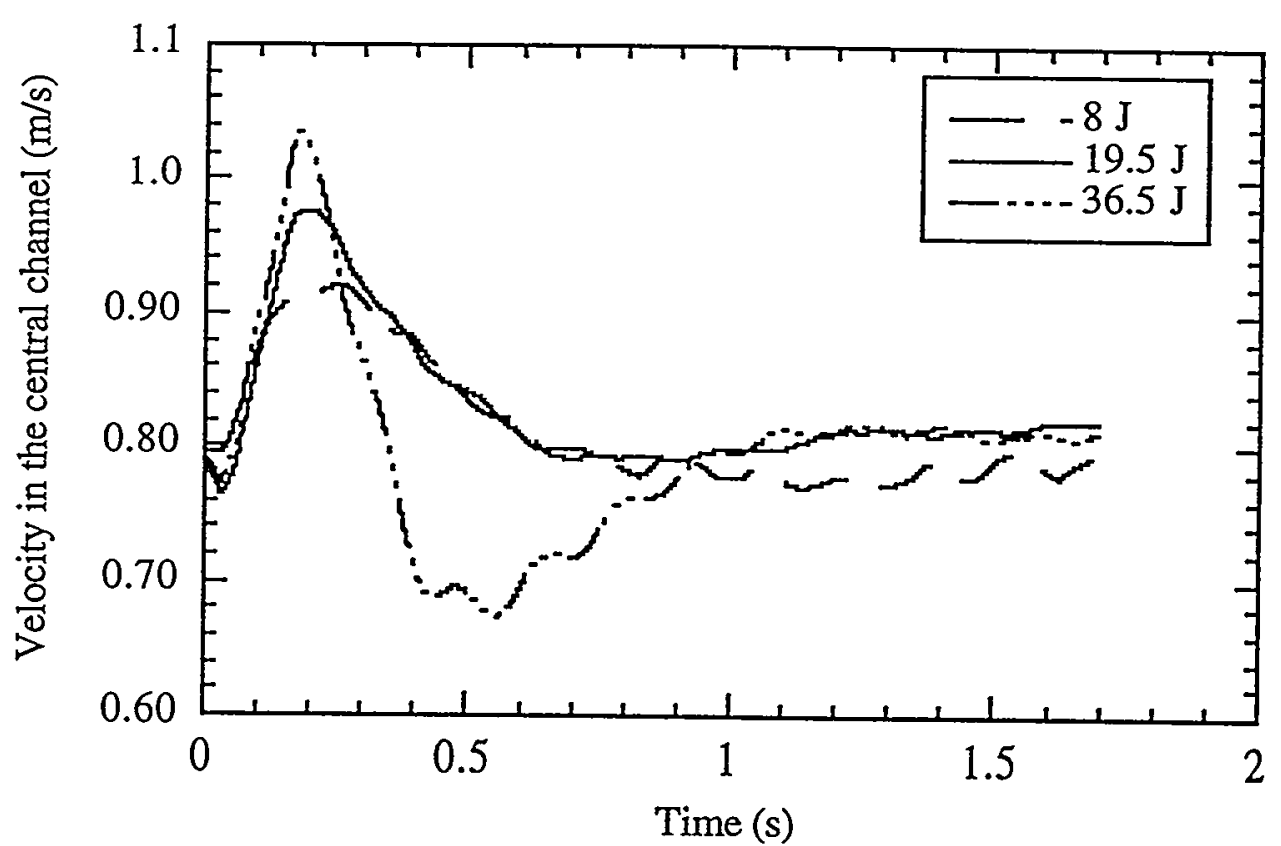

Figure 3.39a. Induced flow velocity in the central channel $(\mathrm{Re}=5100)$.

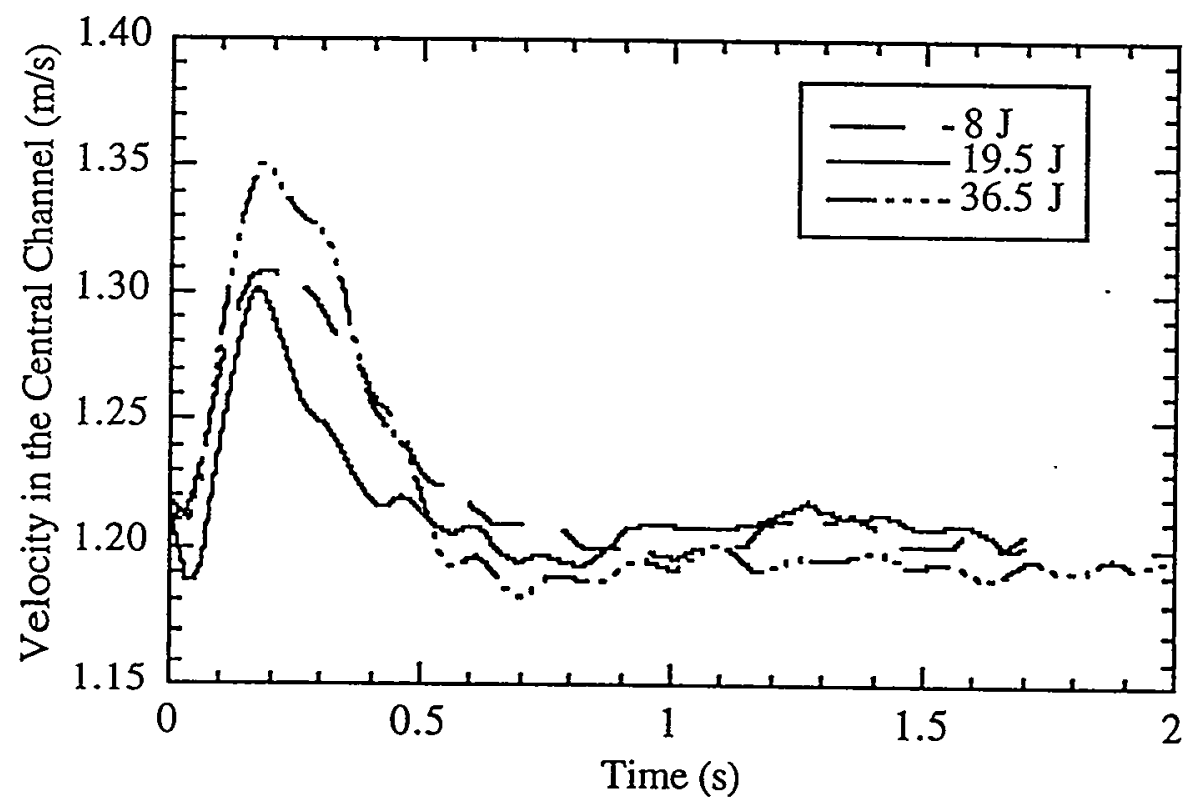

Figure 3.39b. Induced flow velocity in the central channel $(\operatorname{Re}=7050)$. 


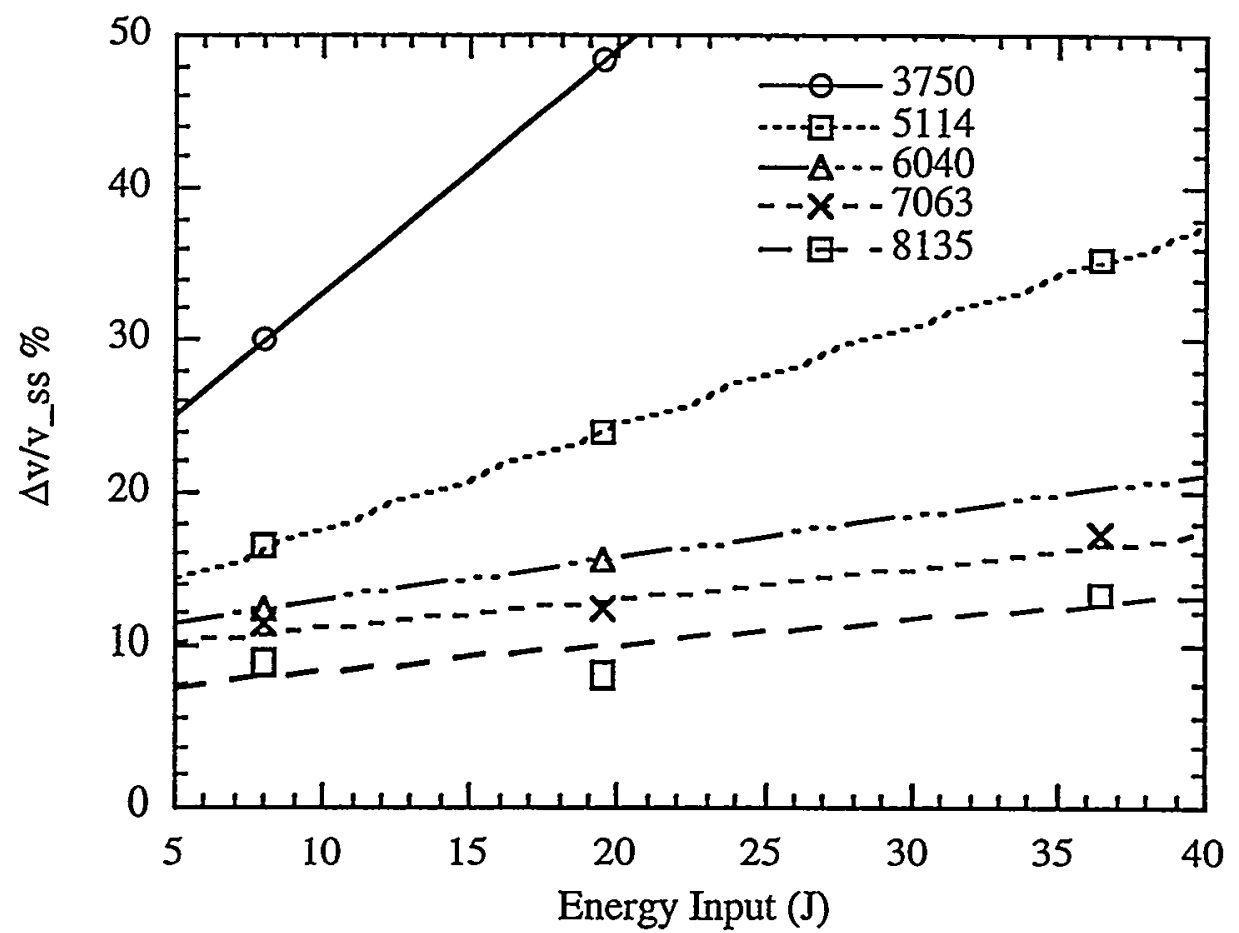

Figure 5.40. The fractional increase of induced flow velocity vs. energy input.

The mass flow rate in the central channel is calculated based upon Equation (5.22). Figures 5.41 (a), (b), (c) show the set of data with different heat inputs. The mass flow in the bundle region is seen to decrease with increasing heat input. It is unlikely that a flow stagnation or reversal is induced in the bundle since the helium expands as absorbing the heat from the strands. Figure 5.42 shows the velocity variation in the central channel and in the bundle. The rapid acceleration and deceleration of the induced flow in the central channel significantly affects the velocity profile in the bundle. Although the central channel mass flow rates were calculated with the measured velocity as an average, the velocity may be its maximum. In addition, since the induced flow is non-steady, the velocity profile may not reach the fully developed flow. The increasing central channel velocity distorts the 
equilibrium at the interface between the central channel and bundle which may also increase the drag forces to change the velocity profile in the channel.

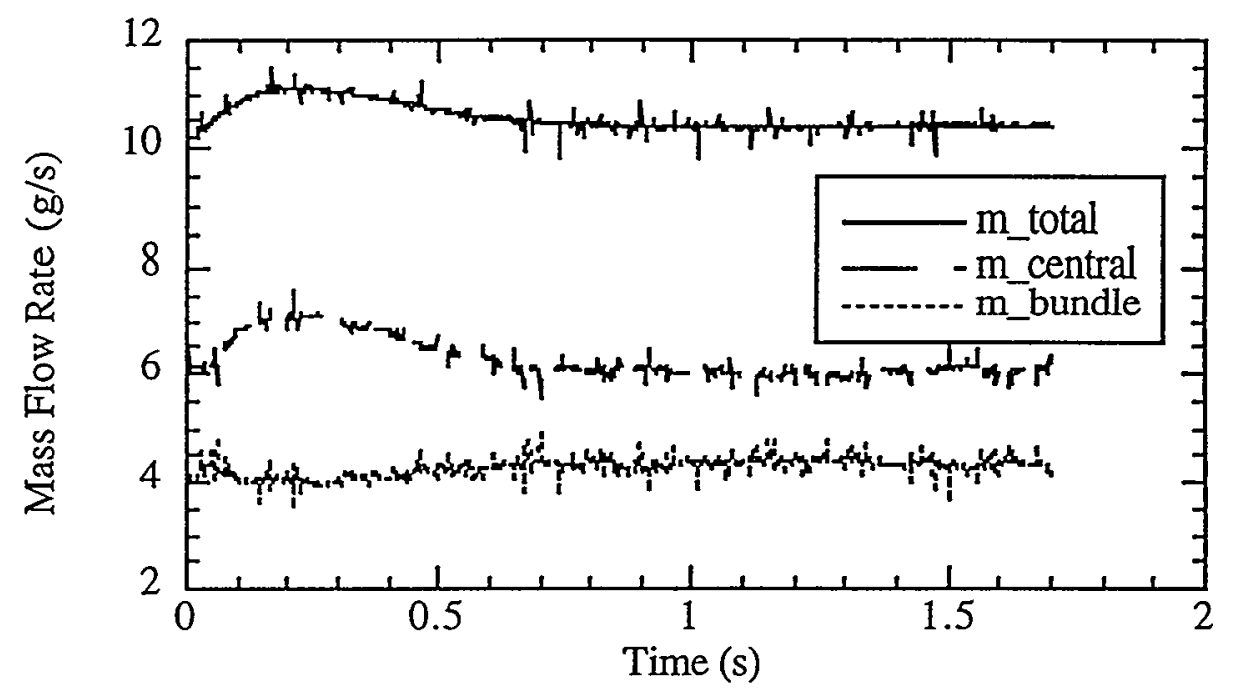

Figure 5.41a. Comparison of mass flow rate in each region (8 J).

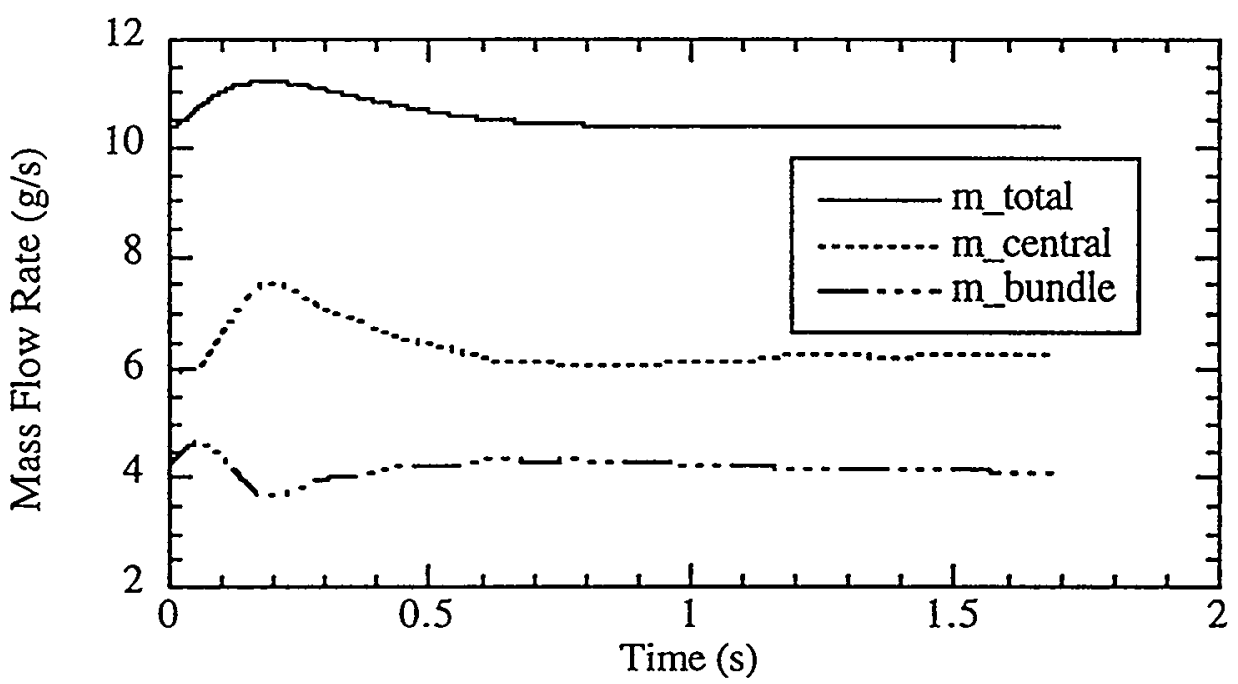

Figure $5.4 \mathrm{lb}$. Comparison of mass flow rate in each region (19.5 J). 


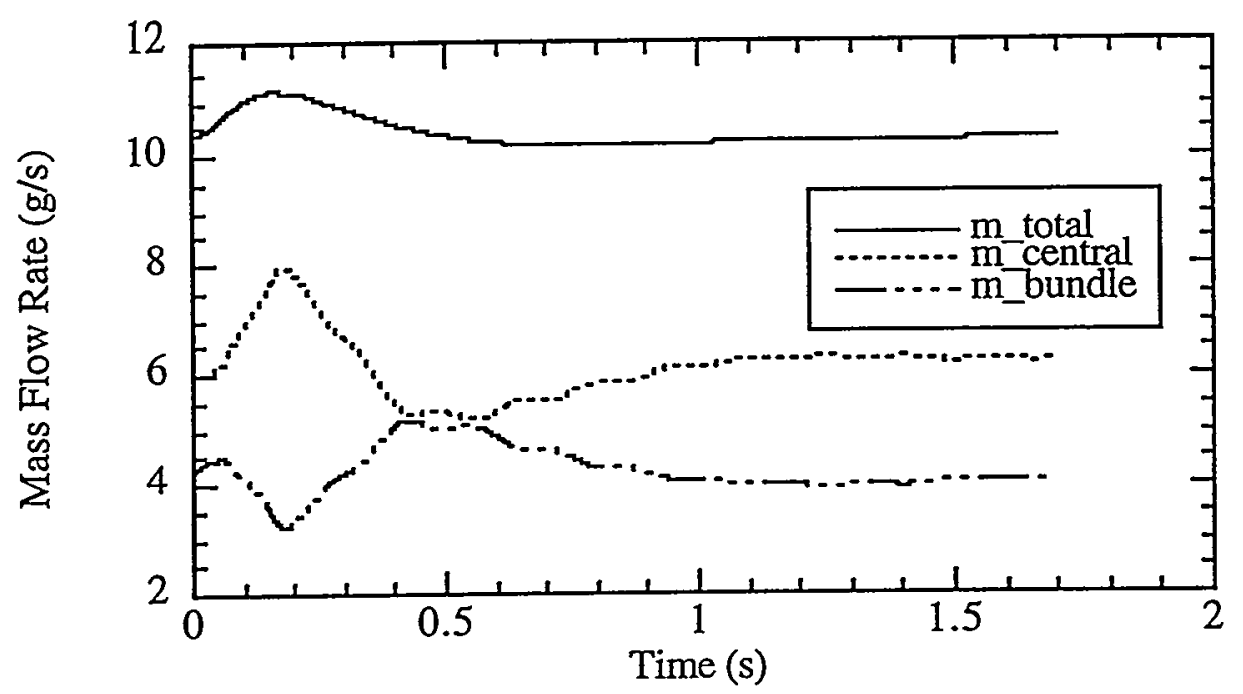

Figure 5.41c. Comparison of mass flow rate in each region (36.5 J).

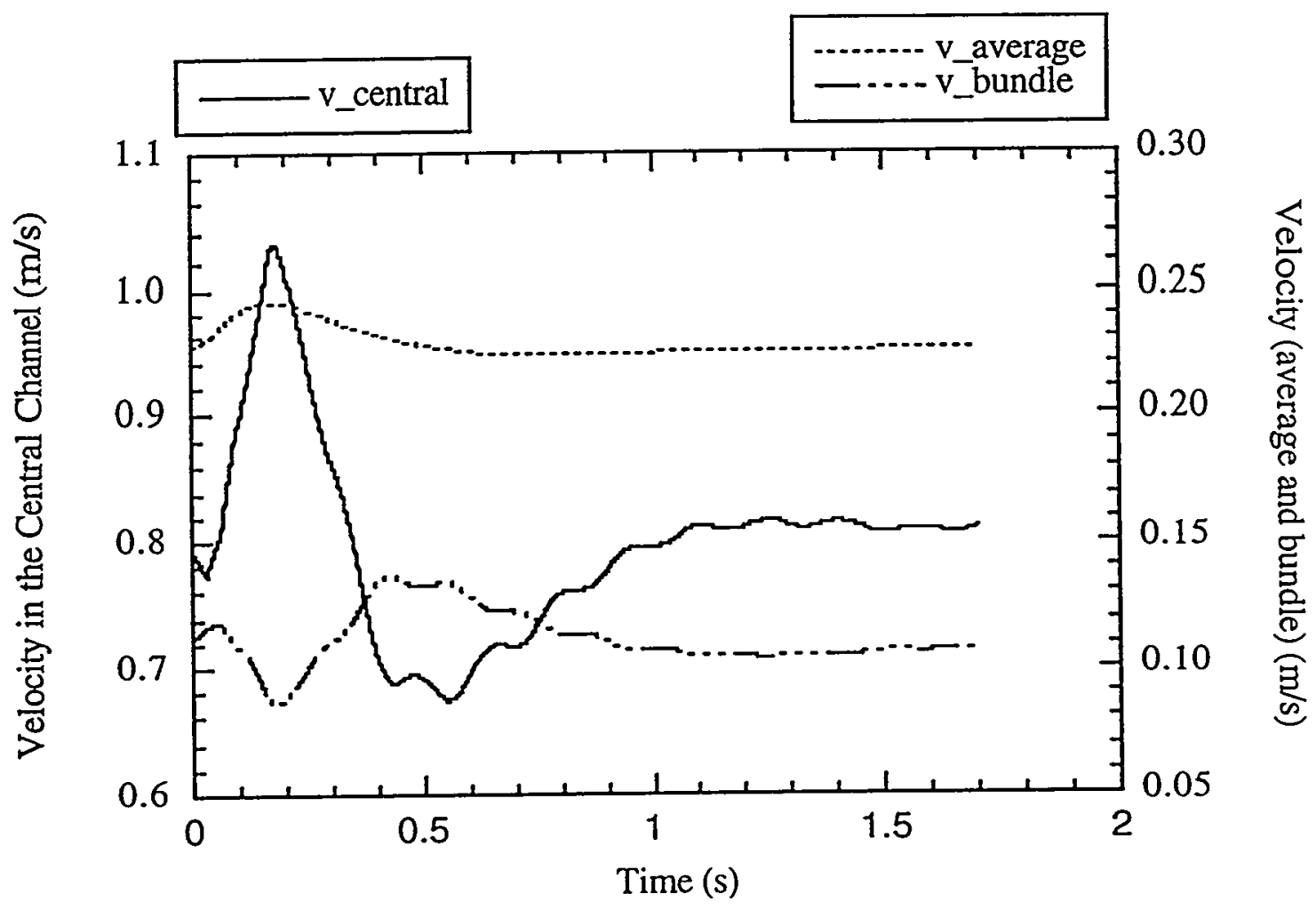

Figure 5.42. Comparison of velocity in each region (36.5 J). 
Pressure wave propagation was measured with five pressure transducers. As helium absorbs the heat, a pressure wave propagates at the speed of sound through the conductor. The pressure difference along the conductor induces bulk flow that has a square root dependence with pressure increase. Figures 5.43 (a) and (b) show the data with pressure increase proportional to the energy input. As pressure reaches a steady value, the induced flow velocity becomes zero.

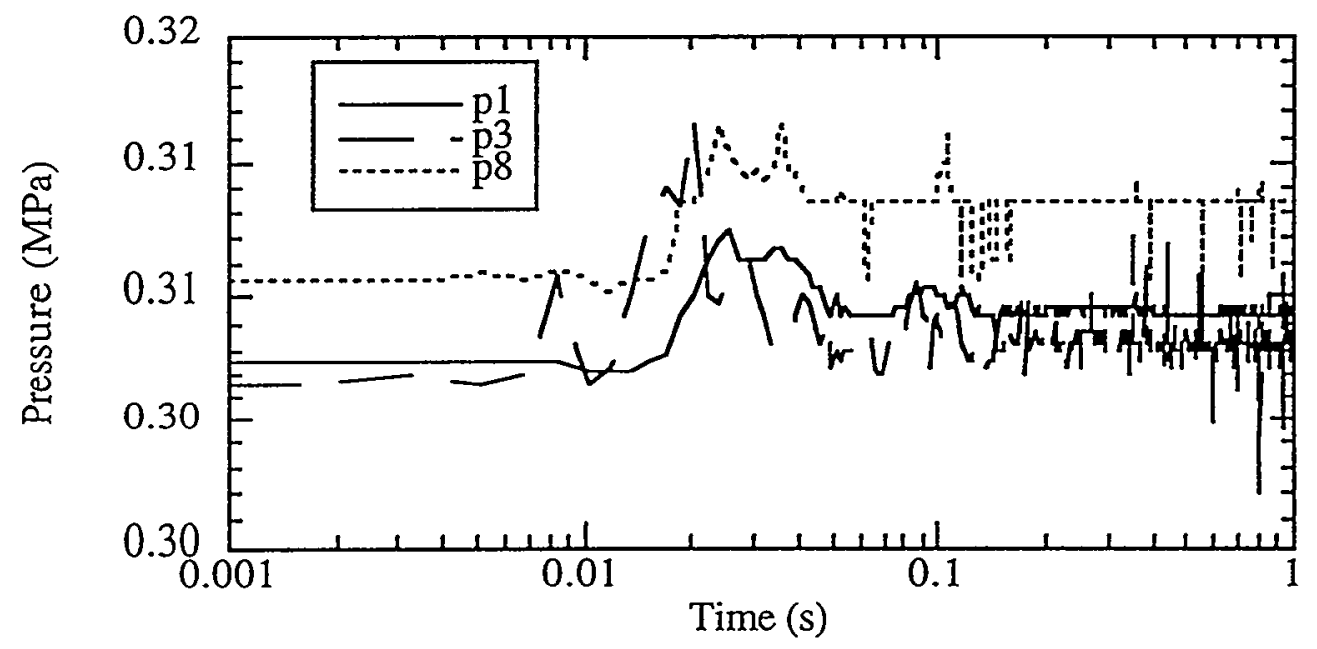

Figure 5.34a. Static pressure change along the conductor after heat pulse (19.5 J).

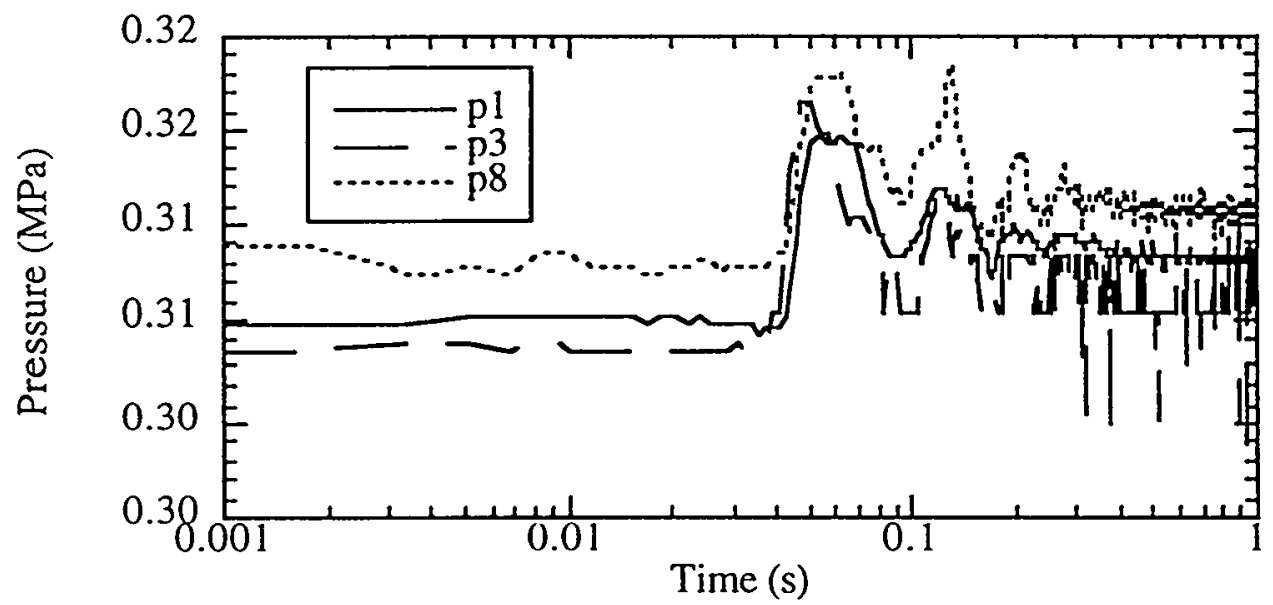

Figure 5.34b. Static pressure change along the conductor after heat pulse $(36.5 \mathrm{~J})$. 
Some reversal of flow in the central channel was recorded at the end of deceleration. This effect may be caused by the pulsating flow in the central channel; i.e. a superimposed periodic pulsation to the steady mean flow. The effect becomes significant as $d p / d x$ changes rapidly; large pressure increase at the heated section. S. Uchida ${ }^{49}$ solved the modified Navier-Stokes equation that shows some reversal of flow in oscillating pipe flow. Although the induced flow was driven by a single heat pulse, the helium expansion upstream of the conductor reduced the imposed flow. This may cause the reduction of flow for a short moment, which is similar to the reciprocating piston in the central part of the conductor. According to the experimental results, flow reversal becomes more significant with increasing heat input. This implies that the helium expansion upstream also affects the flow in the central channel.

Thermally-induced flow of supercritical helium was primarily generated in the central channel because of low flow impedance. The total mass flow rates were not significantly affected by the different energy inputs. Increasing the imposed mass flow rate reduced the drastic induced velocity change in the central channel since enhanced cooling lower the helium temperature increase. Some reversal of flow were recorded at the end of deceleration which is similar to the pulsation flow in the tube.

\subsubsection{Induced Flow with the Thermometer Probe in the Central Channel}

The supercritical helium temperature in the central channel of the CEA conductor was measured with a probe which consisted of eight thermometers in the $5 \mathrm{~mm}$ diameter PVIS06 insulating tubre. Although the probe significantly increases the flow impedance, the experiment simulates thermal hydraulic characteristics of a conductor with a small helium passage in the central channel. The experiments were performed with Reynolds number 
2000 17000. The supercritical helium temperature was about $4.5 \mathrm{~K}$ and pressure was $\sim 0.3$ MPa. The total heat applied to the conductor was varied; $36.5 \mathrm{~J}, 58.3 \mathrm{~J}$ and $82.0 \mathrm{~J}$.

Figure 5.44 shows a typical set of data: the total mass flow rate and average supercritical helium velocity in the conductor versus time. The number beside the legend indicate a total heat input to the conductor. The steady state Reynolds number was around 6000 . The results show the sinusoidal pattern of the induced mass flow. The difference between the maximum and the minimum induced flow becomes larger with increasing heat input from $36.5 \mathrm{~J}$ to $58.3 \mathrm{~J}$. The flow reduction is assumed to be caused by the helium expansion upstream. This effect becomes more significant with high imposed mass flow rate, see Figure 5.45. The phenomenon was not seen in the CEA conductor without having the thermometer probe in the central channel.

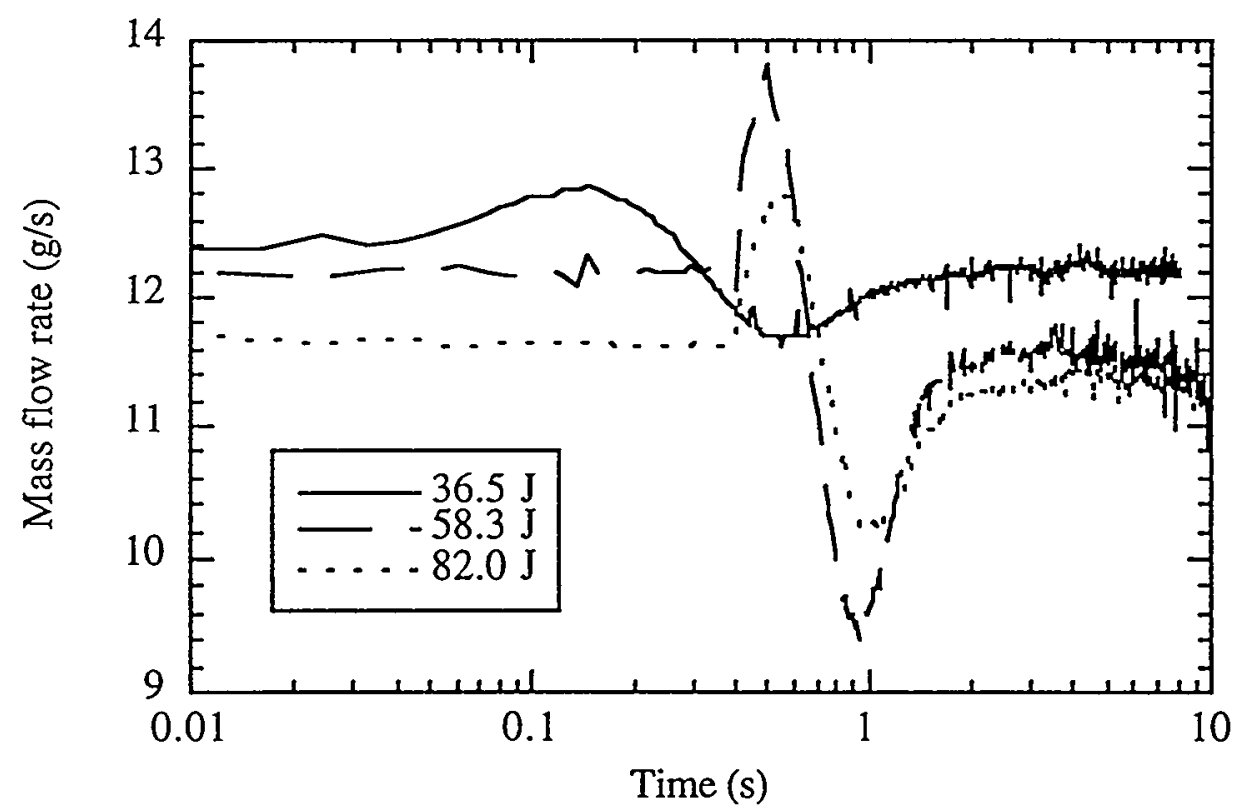

Figure 5.44. Induced mass flow with different heat input $(\operatorname{Re} \sim 6000)$. 


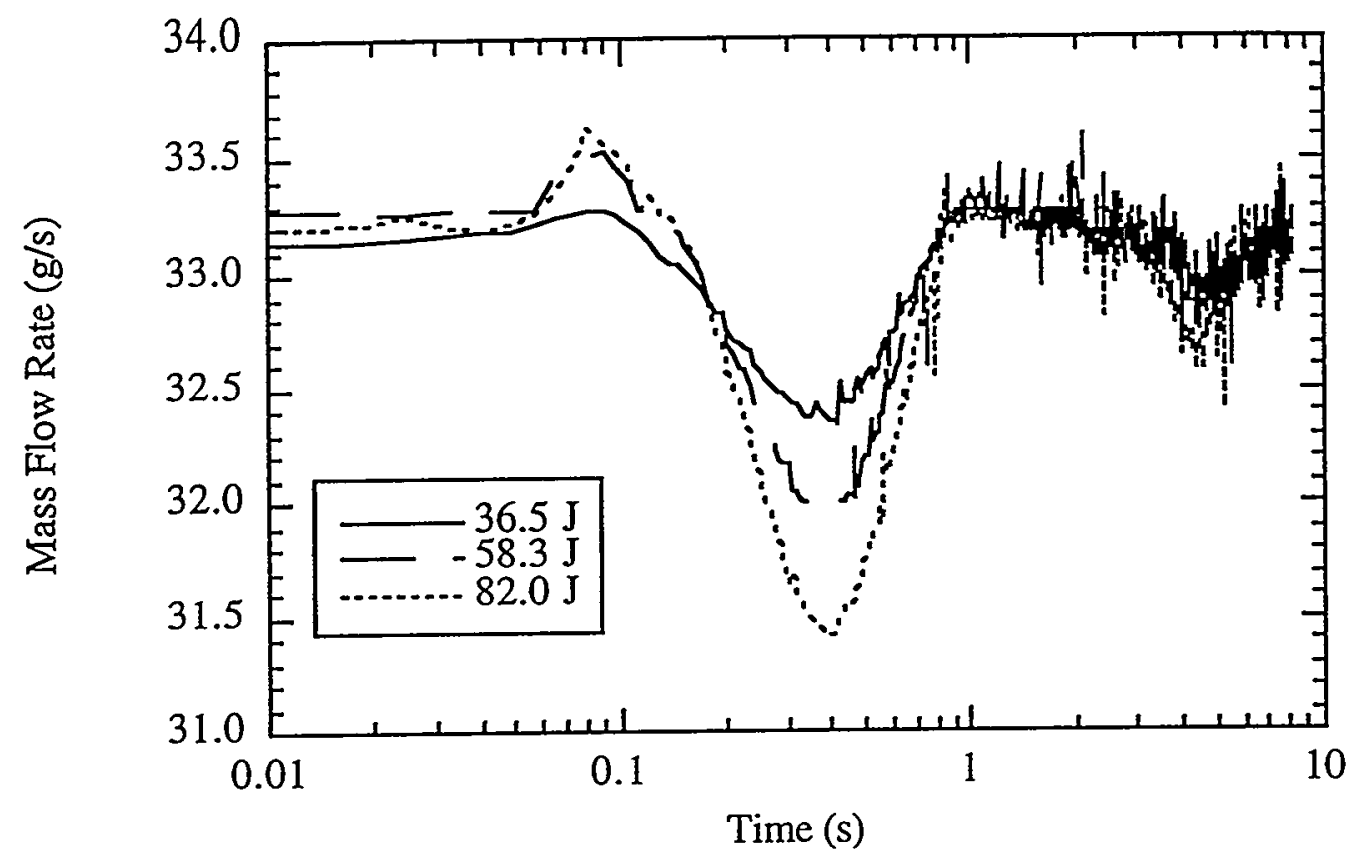

Figure 5.45. Induced mass flow with different heat input (Re 17000).

The basic scenario of this phenomenon is as follows: first, the thermally induced flow is not a linear function of a heat input but rather asymptotic as shown in Figure 5.46. When the heat is input to the conductor, the helium absorbs it and expands longitudinally in both upstream and down stream directions. As discussed in the previous section, the acceleration of fluid in the central channel increases with heat input. However, in this case, the central channel is mostly blocked with the probe, and the expanded helium can not flow smoothly to the down stream end because of high flow impedance in the bundle region. At the same time the expanded helium upstream creates a bulk flow resistance in the loop. Therefore, the flow resistance to the bulk flow is a combination of these effects. Consequently, it leads to the flow reduction of the system. 


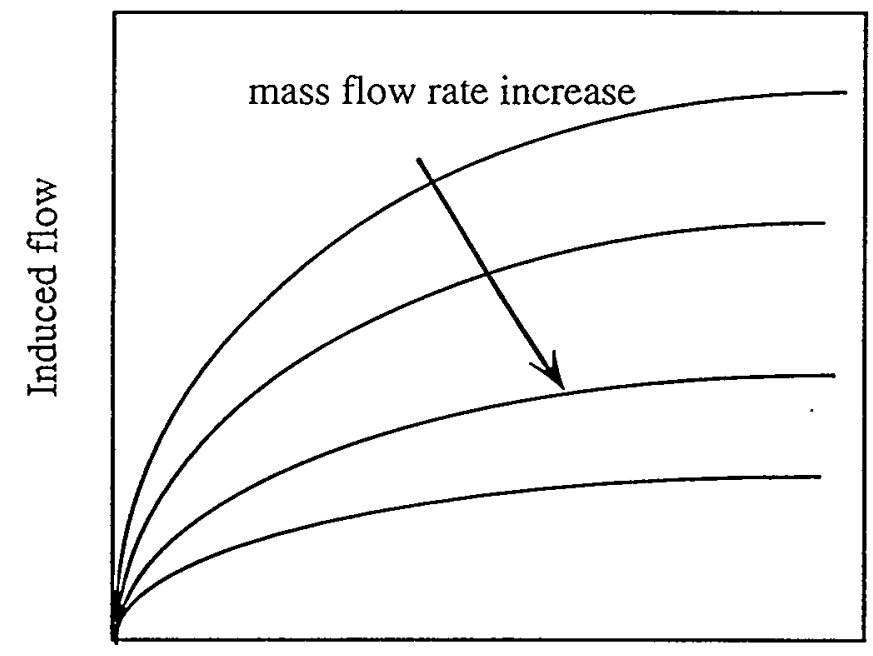

Energy input

Figure 5.46. The relationship between induced flow and mass flow rate.

Figure 5.47 shows the ratio of the maximum induced velocity and a steady state average velocity with different heat input and the mass flow rate. As Reynolds number becomes 5070 or higher, the ratio becomes close to unity. This suggests that the induced flow rate has a certain limit that is not significantly affected by the imposed flow rate. Figure 5.48 shows the ratio of flow reduction velocity and the average velocity. As flow rate increases, the flow reduction becomes smaller and is less dependent on the heat input. 


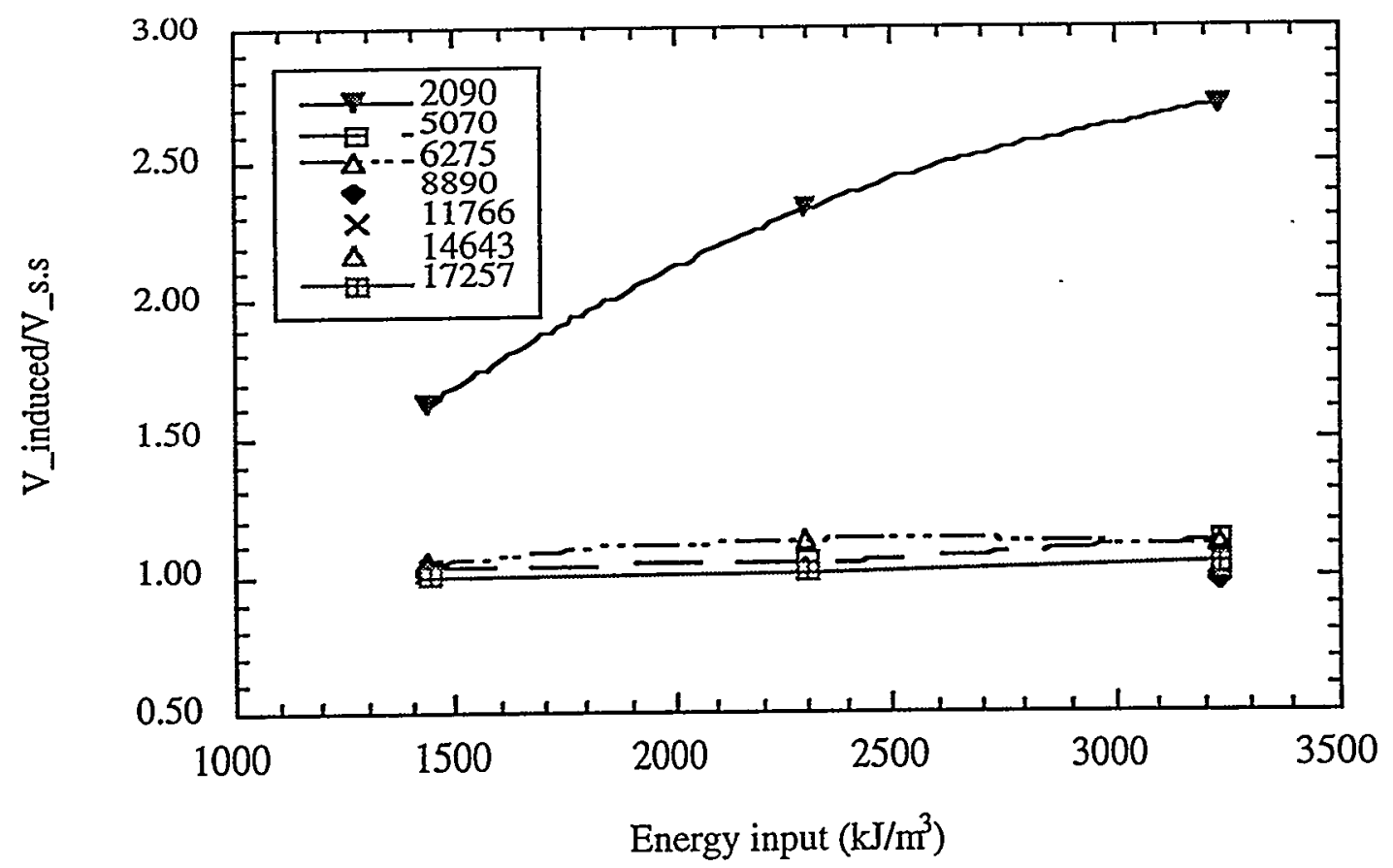

Figure 5.47. The relation of induced velocity to steady state velocity as a function of heat input and imposed mass flow rate.

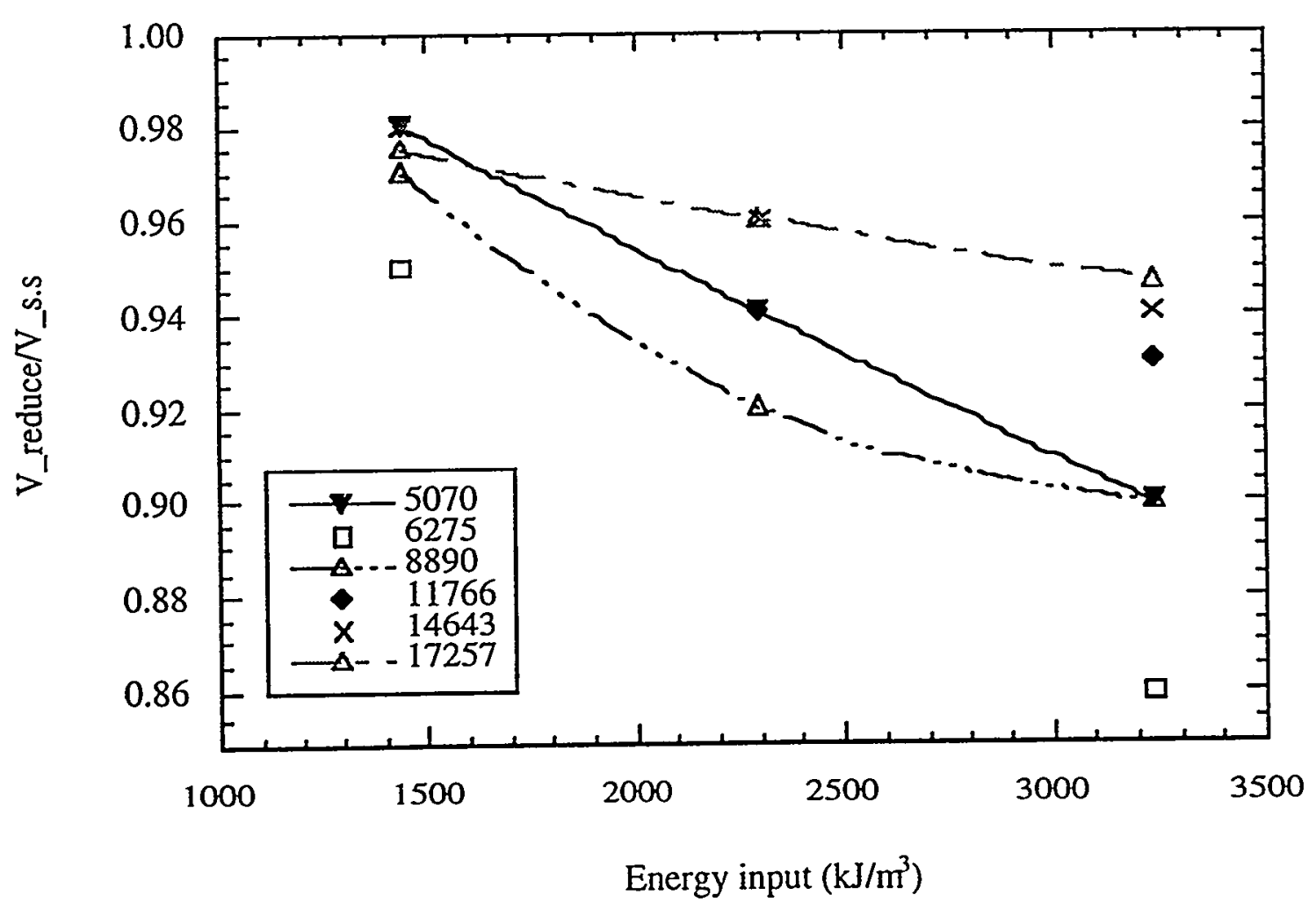

Figure 5.48. Fraction of flow rate reduction with different mass flow rate and heat input. 
Four thermometers are placed at about $0.38 \mathrm{~m}$ intervals except $\mathrm{T} 1$ which is placed about $0.26 \mathrm{~m}$ from the inductive heater. The temperature profile is shown in Figures $5.49 \mathrm{a}, \mathrm{b}$ and c with three different Reynolds numbers calculated in the unperturbed flow condition. Increasing the mass flow rate reduces the time to reach the steady state because of improved heat transfer. Although the pulse arrival time at the thermometer 1 is fast, the induced flow decelerates among position 1 and 3 and accelerates again from position 3 to 4 .

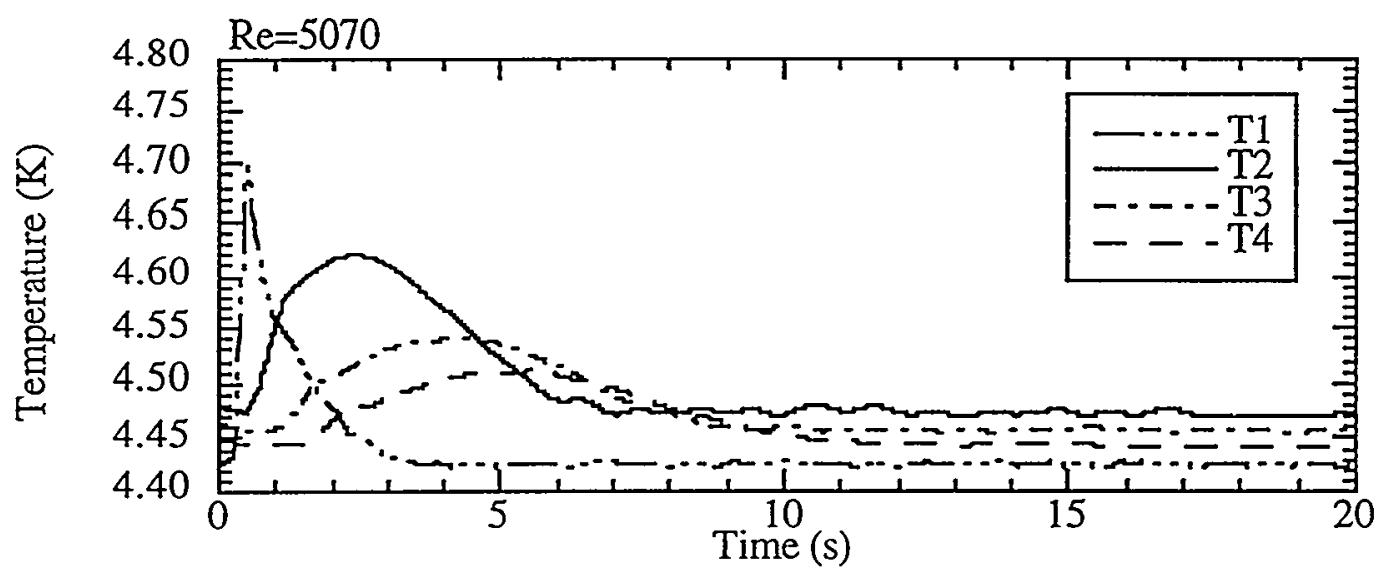

Figure 5.49a. Temperature profile in the central channel (36.5 J).

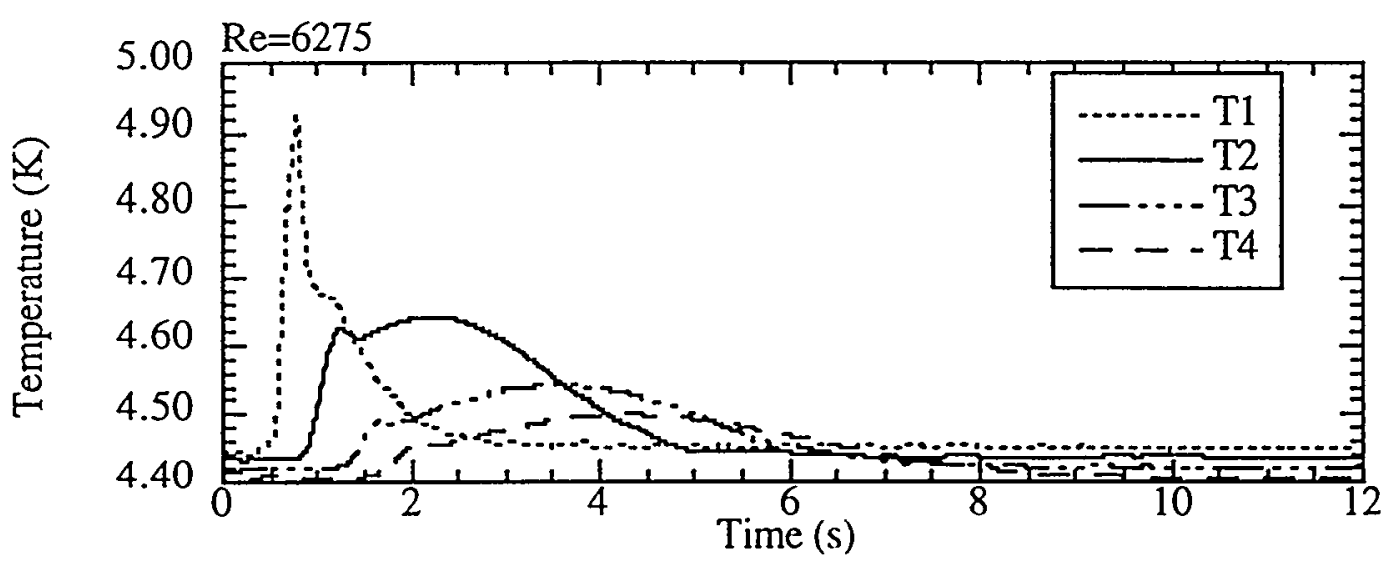

Figure 5.49b. Temperature profile in the central channel ( $36.5 \mathrm{~J})$. 


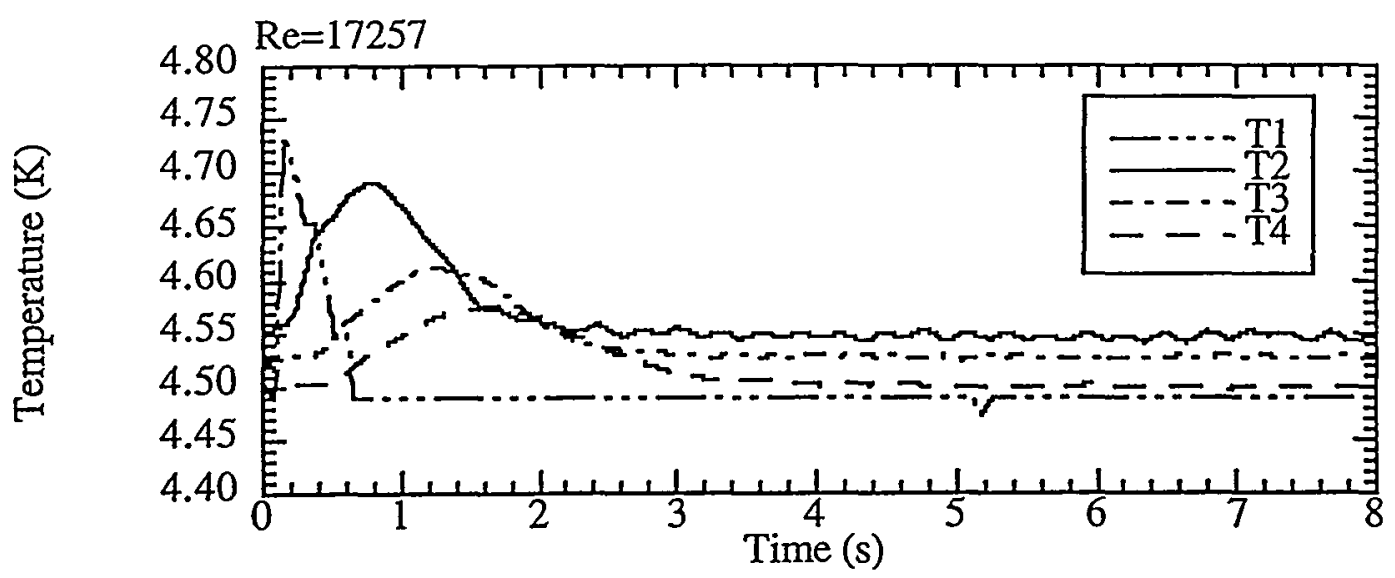

Figure 5.49c. Temperature profile in the central channel (36.5 J).

The induced helium velocity to the down stream was calculated by the time of flight method. Even though the temperature profile changes with traveling to the down stream, a rough estimation of flow velocity iss possible. The average helium velocity at each position were calculated as,

$$
v=\frac{l}{\Delta t}
$$

where $l$ is distance between thermometers and $\Delta t$ is the time difference at which the peak temperature registered at each thermometer. The induced flow velocity from the heater to the position 1 was calculated with Equation (5.2), see Figure 5.50. The calculated velocity with different heat input were approximately two time larger than the induced average velocity recorded at the venturi. Although most of the central channel is blocked by the probe, induced flow velocity in the central channel is still faster than average velocity. 


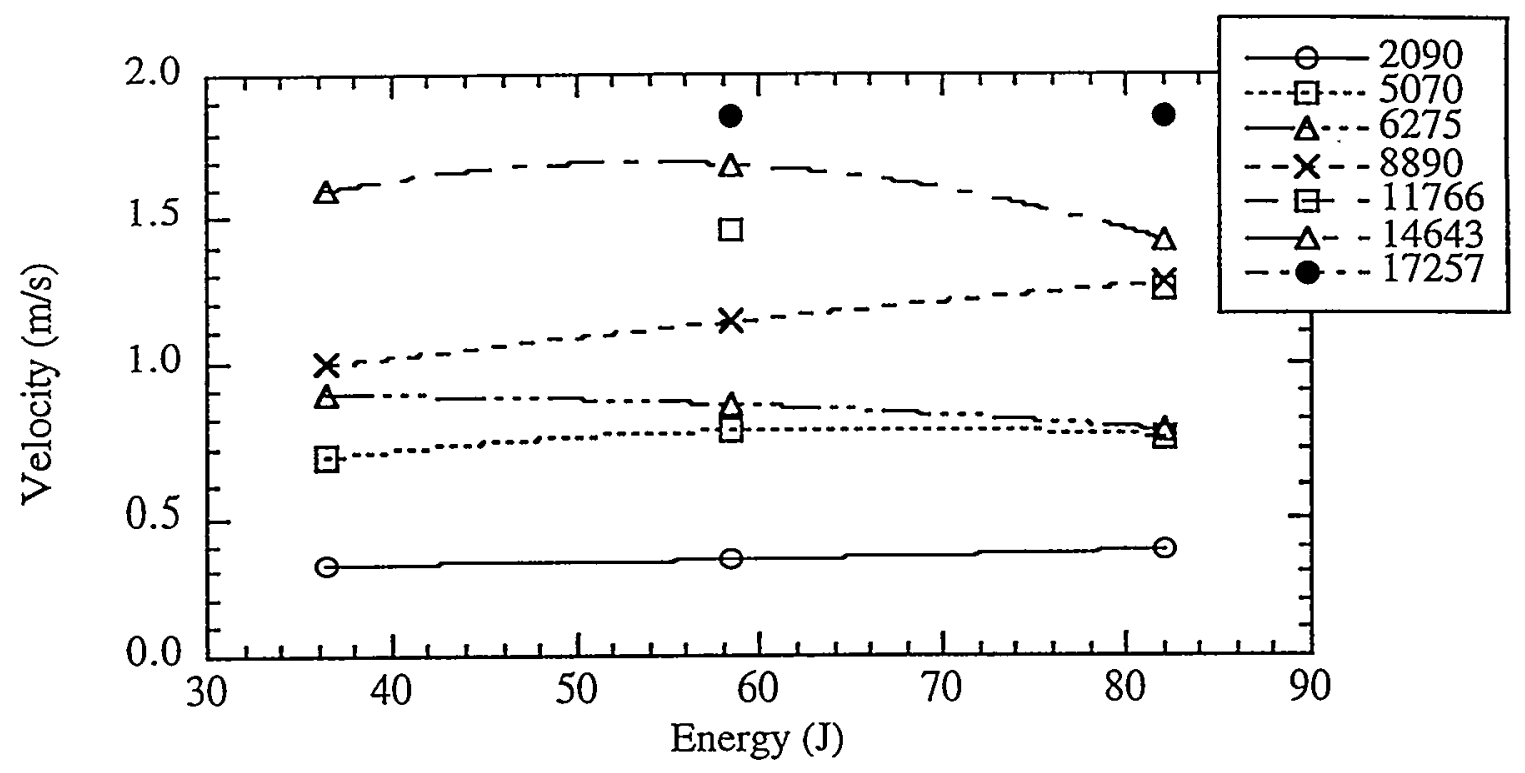

Figure 5.50. Induced flow velocity calculated by time of flight.

Figure 5.51 shows the calculation results for the helium velocity with three different Reynolds numbers and heat input. The average velocity at each position was divided by the maximum velocity which is recorded at position 1 . The results also indicate the flow reduction in the system. The velocity profile in the down stream is independent of mass flow rate and energy input with one exception recorded at the maximum imposed flow rate and the energy input. Even though the reduction of flow is high, the overall pumping power can recover its original state faster than at the lower mass flow rate. Thus the velocity at position 4 with $R e=17257$ shows its maximum value.

The thermal hydraulic characteristics of the hybrid conductor is significantly changed when the central channel area is reduced. The most affected quantity is the reduction of overall mass flow rate. In large system applications, this leads to the refrigeration capacity. In the case of superconducting magnet this can have devastating effects on the entire conductor performance. However, this comparison of the central channel effects to the 
thermal hydraulic characteristics of new type conductor adds credibility to the importance of the hybrid cooling passage.

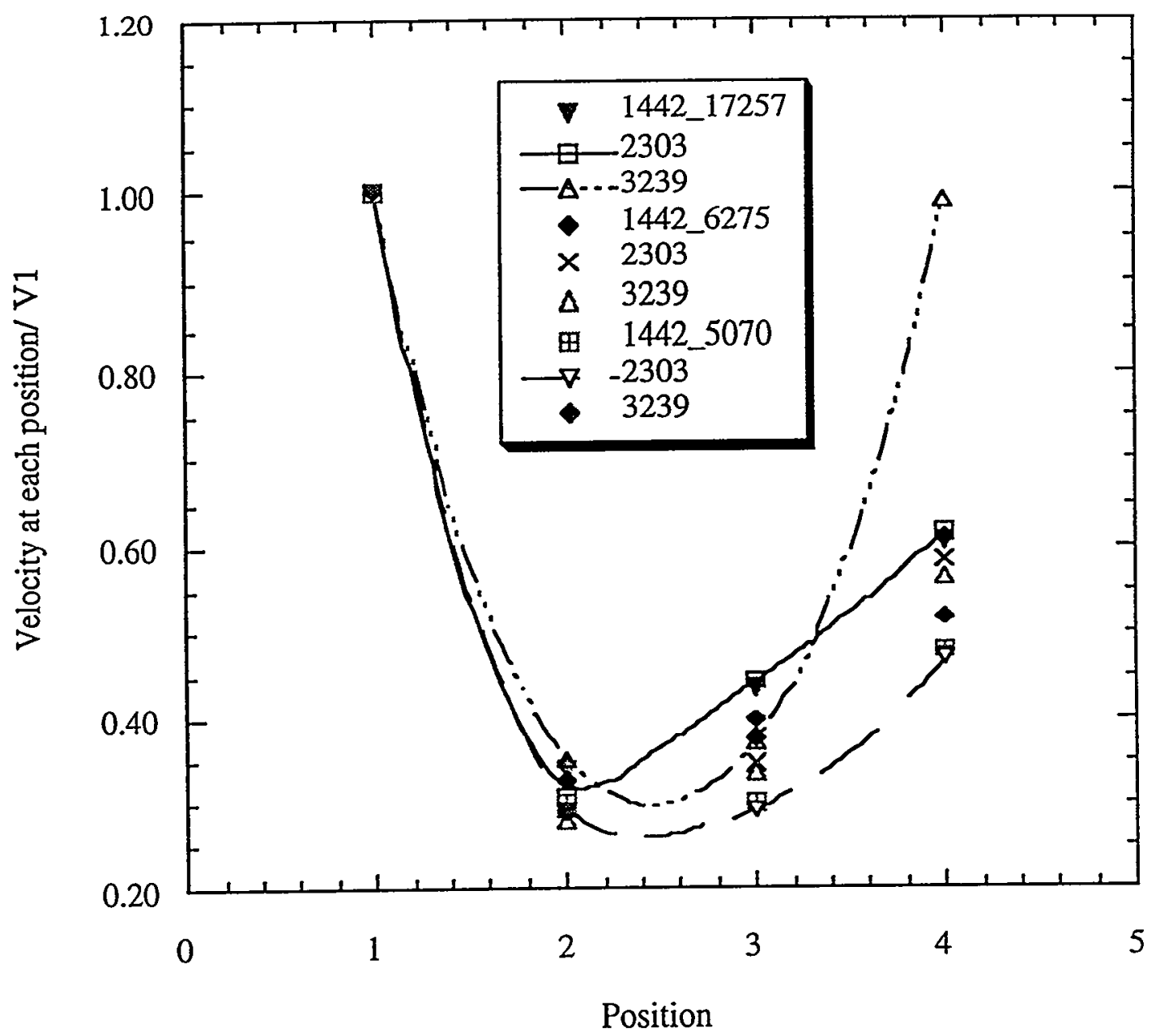

Figure 5.51 . Velocity along the conductor calculated by time of flight. 


\subsection{Transient Heat Transfer Experiment}

The transient heat transfer characteristics of the CEA conductor have been studied, using a short section of the conductor filled with either static He II or supercritical helium. The total heat input to the conductor was varied between $5.12 \mathrm{~J}$ to $27.35 \mathrm{~J}$ with the helium temperature

and pressure initially set at either $1.8 \mathrm{~K}, 1.7 \mathrm{kPa}$ or $4.2 \mathrm{~K}, 0.35 \mathrm{MPa}$. The average heat transfer coefficient is calculated by

$$
\bar{h}(t)=\frac{Q(t)}{A\left(T(t)_{\text {bundle }}-T(t)_{c e n t r a l}\right)}
$$

where

$$
A=\pi D *(\# \text { of strands }) * L_{\text {ind }}
$$

and $L_{\text {ind }}$ is the length of inductive heater. The heat input to the conductor is assumed to be confined within the region of the inductive heater, about $0.05 \mathrm{~m}$ long.

\subsubsection{Saturated He II}

Since He II has extremely high thermal conductivity, the test section must be isolated from He II bath just before the heat pulse. Figure 5.52 shows a typical result for the temperature profile in both the bundle region and central channel as the result of a heat pulse to the conductor. Note that the heat pulse starts at $0.09 \mathrm{~s}$. The bundle temperature starts to increase simultaneously with the heat input. However, T2o, which is located at $0.03 \mathrm{~m}$ away from the heater. does not show any changes until the end of first pulse. Similarly, the He II temperature is constant until the end of first heat pulse. This is primarily caused by the short 
time interval of the pulse $\sim 20 \mathrm{~ms}$. It takes about one second to reach the equilibrium condition.

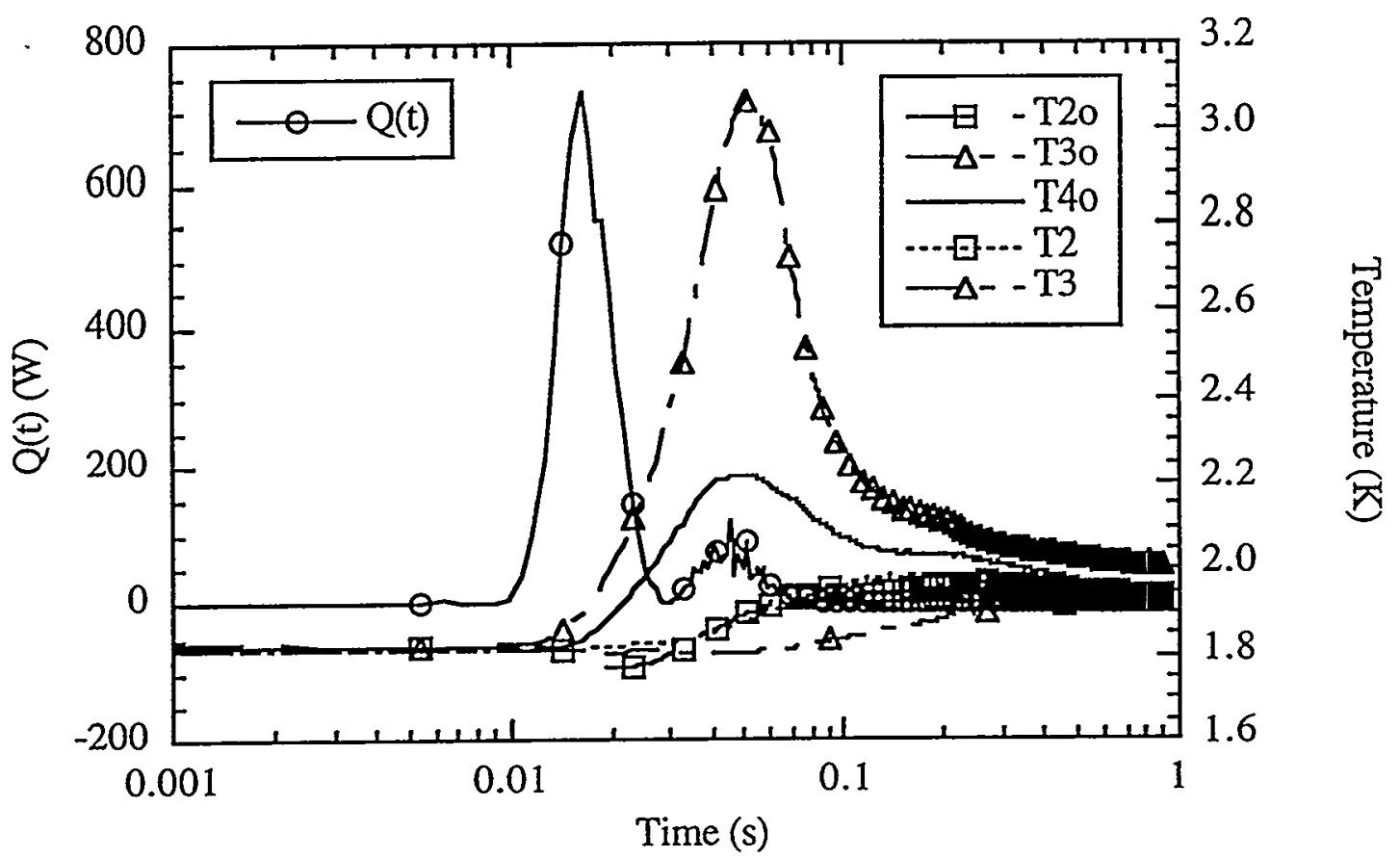

Figure 5.52. Temperature profile after the heat pulse (He II).

To calculate the heat transfer coefficient between He II and copper strands, several assumptions are made. The transverse temperature difference is taken to be negligible because of high effective thermal conductivity of He II. Also, the heat transfer area is calculated based upon the geometric analysis and the axial heat transfer is assumed to negligible because of short time scale. Further, the measured bundle temperature is assumed to be an average temperature of copper strands and He II. In general, the enthalpy change of copper is taking into consideration; however, it is negligibly small, less than $1 \%$, compared with total heat input to the system.

Based on these assumptions, the heat transfer coefficients are calculated, using Equation (5.22). Figure 5.53 shows the calculated heat transfer coefficient for three different heat 
inputs: $5.1 \mathrm{~J} ; 8.2 \mathrm{~J}$ and $12.4 \mathrm{~J}$. Although each heat transfer coefficient follows the same curve, the calculated value is orders of magnitude higher than that of Kapitza conductance for copper. This may be caused by underestimating the heat transfer area between the copper and He II since it is not an easy task to define how the time varying magnetic field penetrates the conductor. It is also possible that the temperature measurement of the strands is inaccurate.. However, the results show consistency with different heat input which may be used as a standard to compare with supercritical helium.

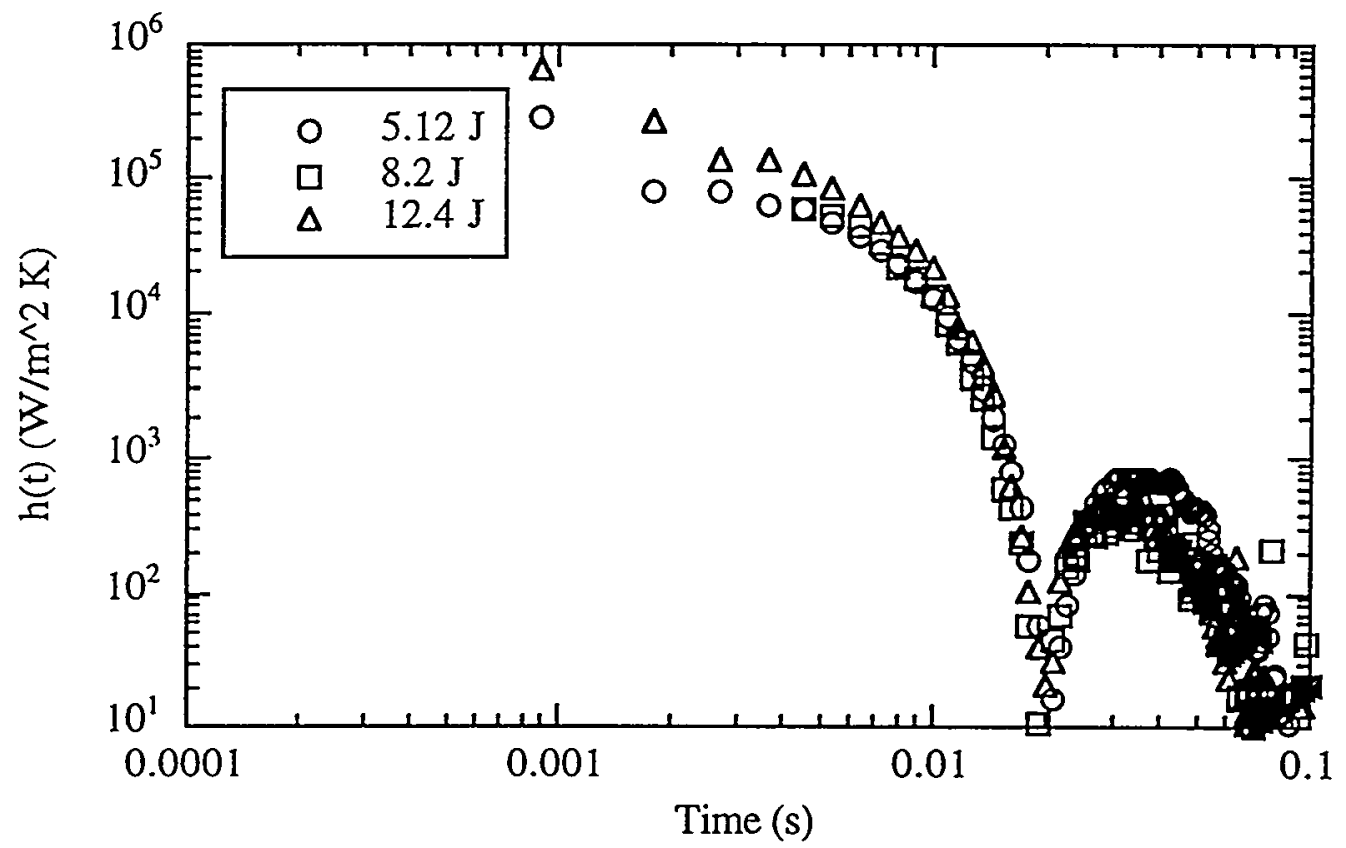

Figure 5.53. Heat transfer coefficient (He II).

\subsubsection{Supercritical Helium}

The same experiments were conducted with supercritical helium with total heat inputs: 13.3 $\mathrm{J} ; 19.7 \mathrm{~J}$ and $27.4 \mathrm{~J}$. Figure 5.54 shows a typical bundle temperature profile with $13.3 \mathrm{~J}$ heat input. First, T3o has two different sloped regions as it approaches the maximum 
temperature. This is caused by the transposed critical temperature of supercritical helium at which the product of density and specific heat has a maximum value. The remarkable thing is that $\mathrm{T} 4 \mathrm{o}$, located at $0.01 \mathrm{~m}$ away from the heater is seen to increase above T3o. Further, this temperature increase is independent of the heat input. This phenomenon is also seen at different heat inputs; however, T4o remains below T3o in He II.

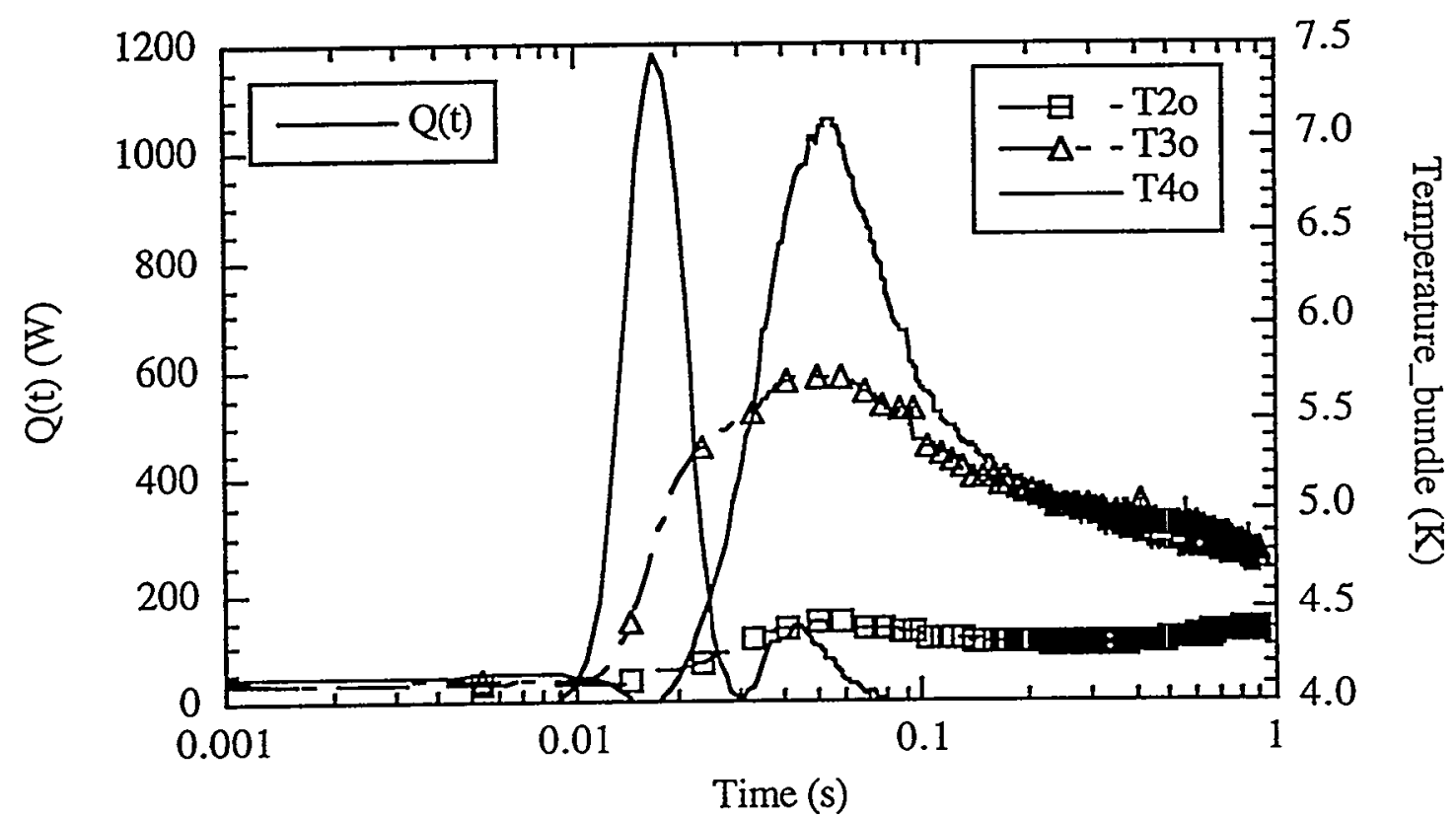

Figure 5.54. Strands temperature profile after heat pulse.

To understand this phenomenon, the supercritical helium temperature in the central channel is also plotted in Figure 5.55. Unfortunately, the figure does not reflect any sign of the highest temperature recorded at T40. Supercritical helium temperature in the central channel shows almost the same value along the conductor. At this point, the reasons for these observations are still unclear.

The average heat transfer coefficient is calculated using T3o. Figure 5.56 shows the result for three different heat inputs. The heat transfer coefficient curves show similar 
profiles to those of He II. The calculated heat transfer coefficient is also over estimated, orders of magnitude larger than the heat transfer coefficient calculated by diffusion heat transfer, as discussed in the section 2.3.3.

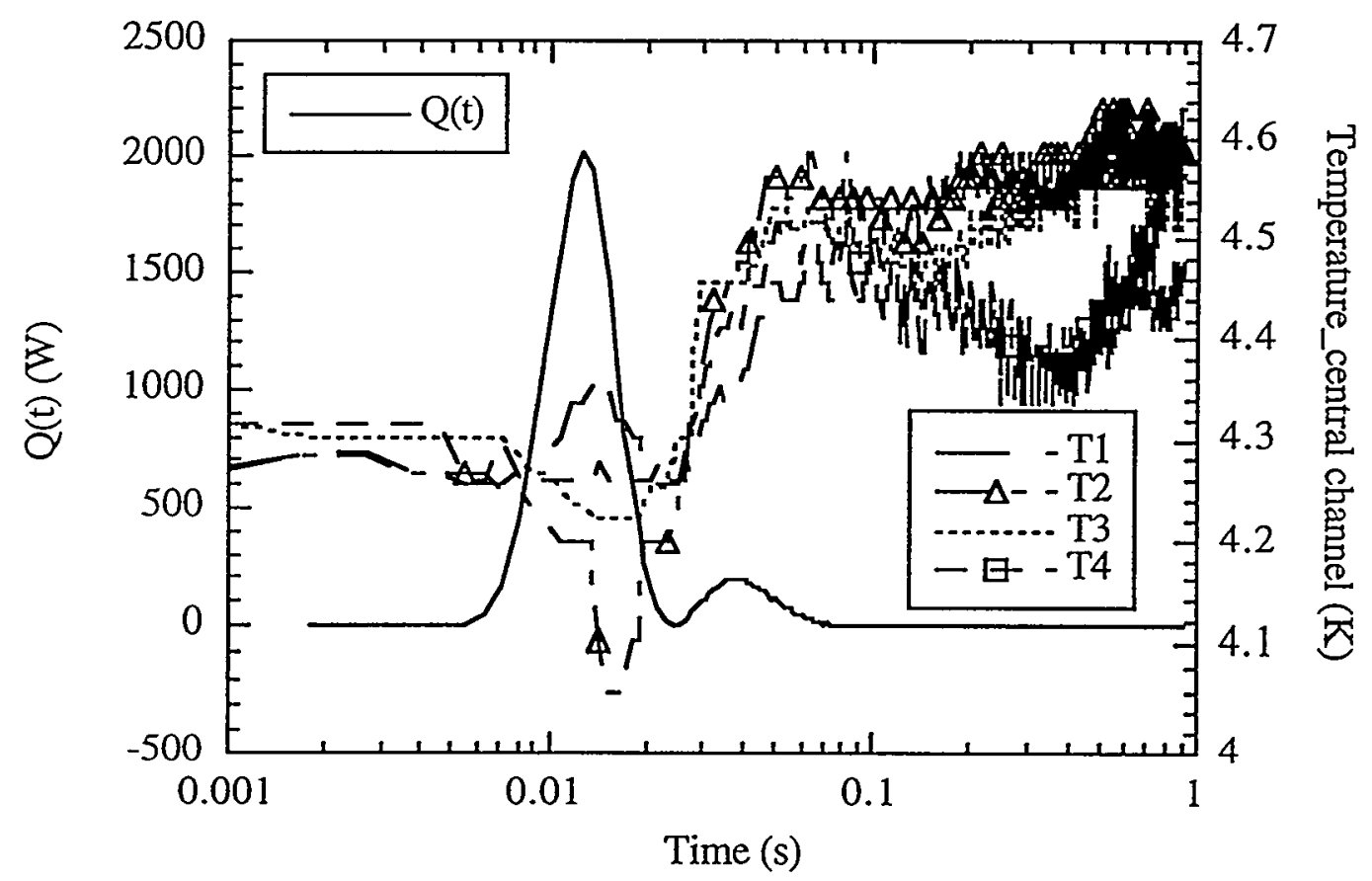

Figure 5.55. Supercritical helium temperature in the central channel.

Although T3o is used as the average strand temperature, the temperature distribution along the conductor is unclear because of behavior of T4o. It is unreasonable to assume that the time varying magnetic field causes the temperature increase at $\mathrm{T} 4 \mathrm{o}$ since the temperature increase does not correspond to the eddy current generation. In addition, the heating by conduction may be question since the temperature becomes higher than T3o. 


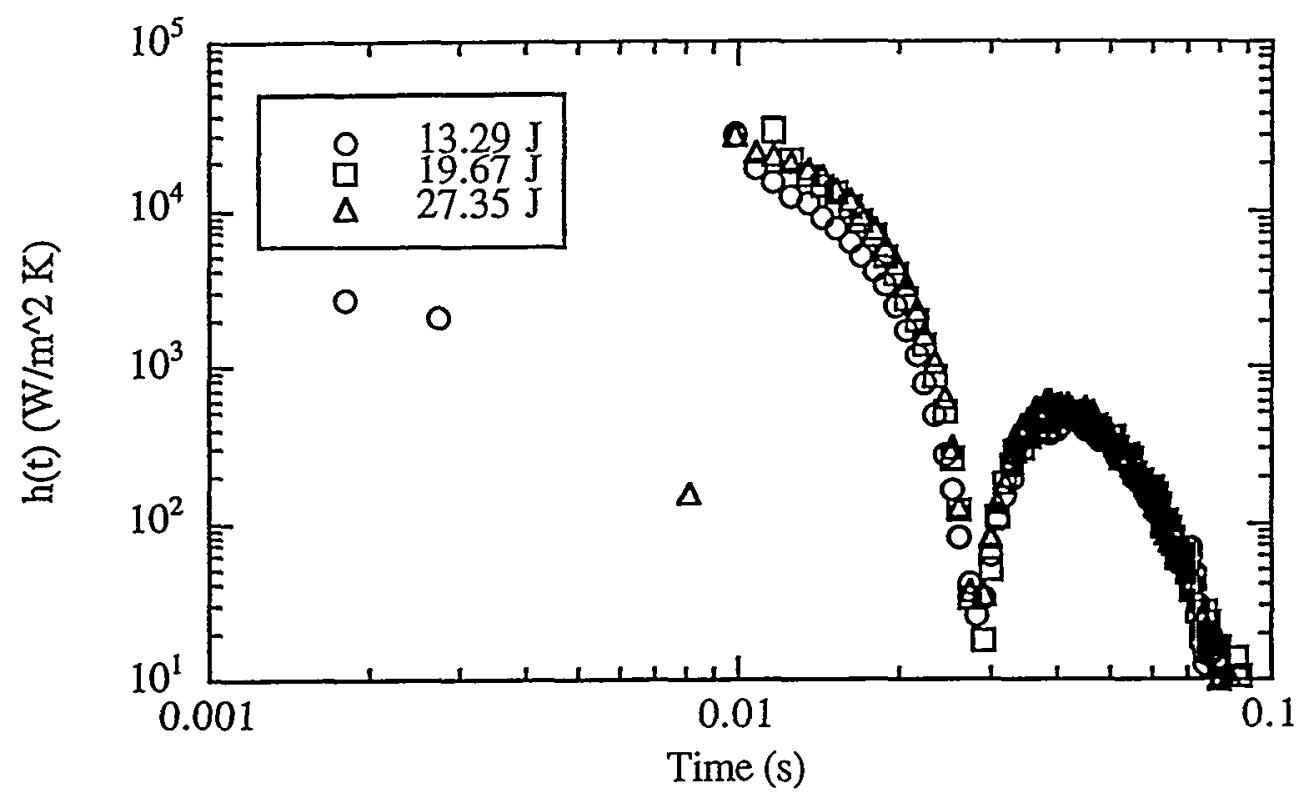

Figure 5.56. Heat transfer coefficient. (supercritical helium).

\subsubsection{Transverse Heat Transfer in the CEA conductor}

Since the conductor has distinct regions: bundle and central channel, the heat transfer between two regions can be modeled based upon the transverse temperature difference of supercritical helium. The scenario assumes that the heat input to the bundle region mostly increases the helium temperature since the specific heat of copper is much smaller than the helium. According to the conductor characterization, the helium volume in the heated region can be calculated. Thus, the enthalpy change of helium is obtained by integrating the product of specific heat and density. The time derivative of this quantity is subtract from the total heat input. The effective heat transfer area is assumed to be the central channel cooled perimeter times the length of the inductive heater. Therefore, the heat transfer coefficient can be obtained from, 


$$
\bar{h}(t)=\frac{Q(t)_{\text {total }}-\frac{d}{d t} H(t)_{\text {Helium }}}{A_{\text {effec }}\left(T_{\text {bundle }}-T_{\text {central }}\right)}
$$

where $A_{\text {effec }}$ is the effective heat transfer area and $\frac{d}{d t} H(t)_{\text {Helium }}$ is the helium enthalpy change.

Figure 5.57 shows the comparison of all heat transfer coefficients obtained by experiment with the calculated heat transfer coefficient,

$$
h(t)=k(\pi \alpha t)^{-1 / 2}
$$

The scatter of the points is primarily caused by the slope change of T3o before reaching the maximum temperature. In addition, the drastic change of helium property around the transposed critical temperature also affect the calculation. The heat transfer coefficient obtained by the model shows the highest values, even though the absolute value of heat transfer between bundle region and the central channel is reduced. This effective heat transfer area is much smaller than that of geometric calculation (about a $97 \%$ reduction),. which significantly affects the heat transfer coefficient calculation.

Although overall heat transfer coefficients obtained here are much larger than known value, see Figure 2.4, the difference in heat transfer coefficient between $\mathrm{He}$ II and supercritical helium is of the order. This suggests that the estimated heat transfer area may be much larger than previously assumed. In addition, the heat input to the conductor may be smaller than the calculation, since it neglects the time constant of the conductor. If time constant is larger, the heat input becomes longer and the maximum value becomes smaller. Some improvement of the effective heat transfer area estimation is also necessary. 


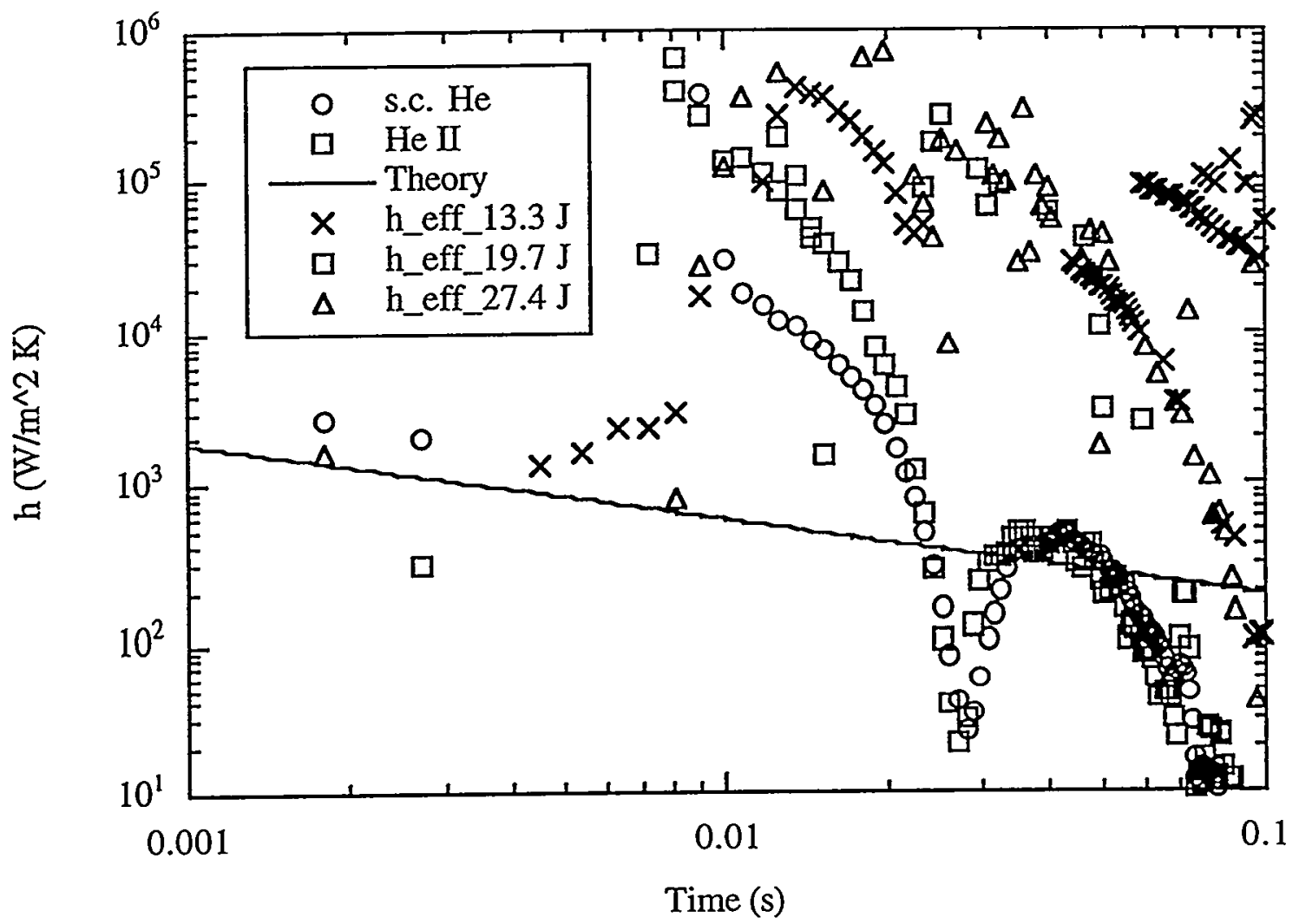

Figure 5.57. Comparison of heat transfer coefficient. 


\section{Conclusions}

\subsection{Hydraulic Characteristics of CICCs}

The reported pressure drop measurements clearly indicate the flow impedance improvement of the CEA conductor with a central channel. The measurements also supported the assumption that the hydraulic characteristics of this new type conductor are dominated by the central channel. Because of this, the classical Blasius turbulent equation for a smooth tube may be used to describe the friction factor and Reynolds number relationship of such a hybrid CIC conductor. It is also found that the fluid velocity distribution in the conductor is entirely different between the central channel and bundle region: the fluid velocity is approximately three times higher in the central channel in high Reynolds number regime.

Flow modeling in the CEA conductor was developed to describe the fluid flow in each region: central channel; six bundles; and six external channels. The hydraulic characteristics of the central channel appear to behave similarly to that of a smooth tube. Although the mixing of flow seems to occur at the interface between the central channel and bundles, the effects may be negligible in the steady state condition. Thus, it is possible to consider the conductor consisting of independent flow passages because of distinct conductor cross section. This type of modeling has not been applied to the conventional CICCs.

Different flow area characterization methods give a range to estimate the friction factor. The image analysis and counterflow analysis are used as an upper bound and lower bound of friction factor variation, respectively. Surprisingly, the nominal geometric calculation gives fairly accurate flow estimation for both NET and CEA conductor. This may suggest that the geometric calculation provides sufficiently accurate values of these parameters for the 
friction factor and Reynolds number calculation. However, it is better to have an image analysis for comparison, since CICCs have complicated configurations. It is not an easy task to estimate the cooled perimeter including a stainless steel strip or a coiled like spring in the central channel.

As a result of these measurements, it may now be possible to characterize the new type of CIC conductor with a central cooling channel, of which CEA is an example. Katherder's correlation seems to provide a reliable estimate based upon the void fraction for conventional CICCs. Although conventional CICCs have been characterized by their hydraulic diameter, that approach can not be applied to flow characterization of the hybrid conductor. Since the central channel has much larger hydraulic diameter than the conductor, it is contradictory to use a smaller diameter for the practical parameter. In addition, it is clear that the void fraction does not significantly affect the flow characteristics since both NET and CEA have almost the same void fraction. A rather important factor is the geometric configuration of the conductor. It is obvious that the central channel greatly reduces the overall pressure drop of the conductor and improves hydraulic characteristics.

\subsection{Thermally Induced Flow in the CEA conductor}

The inductive heating method provides good insight about the thermally induced flow behavior in the CEA conductor. It is evident that the thermally induced flow is mainly generated in the central channel. The acceleration of fluid in the central channel is enhanced with increasing heat input; however, the overall flow is not significantly affected by the heat input. It is found, by using supercritical helium and He II, that the induced flow increases asymptotically with increasing heat input. Therefore, Dresner's analysis may only be used to estimate the expulsion velocity at low heat input. It is also evident that the imposed flow 
suppresses the induced flow because of high heat transfer rate between copper strands and supercritical helium.

Transient pressure wave propagation through the conductor was also recorded as it relates to the induced flow in the conductor. Induced flow is symmetric at the heater since the pressure wave propagates at the speed of sound in both upstream and down stream directions.

At the end of deceleration of the fluid, a reversal of fluid motion is observed in the central channel, similar to that seen in smooth tubes with pulsating flow. This may also suggest that the hydraulic characteristics of the central channel are similar to the smooth tube.

Temperature measurements in the central channel provide convincing support for the significance of the central channel to overall hydraulic characteristics. Thermally induced flow shows entirely different behavior in the central channel with the thermometer probe inserted. Reducing the central channel area significantly increases the flow impedance, which results in total flow reduction in the loop. An imposed flow can not overcome the flow resistance created by both central channel blockage and thermal expansion of supercritical helium to the upstream. Although the flow reduction rates become smaller with higher imposed flow rate, in the case of large scale superconducting magnets, this effect will be crucial to the reliable operation of the magnet.

Throughout these two independent experiments, it is apparent that the locally transient disturbance to the CEA conductor is carried away by the fluid in the central channel. Therefore, the central channel provides significant improvement of both thermal and hydraulic behavior of CIC type superconductors. 


\subsection{Transient Heat Transfer}

The transient heat transfer experiment provides some insight of heat transfer mechanisms that occur in the CEA conductor. Although the calculated heat transfer coefficients are an order of magnitude larger than either the Kapitza conductance or a diffusion heat transfer calculation, the difference between He II and supercritical helium heat transfer coefficient is of the right order. The transverse heat transfer model showed some potential to understand the communication of the helium in the bundle region and in the central channel. However, the effective heat transfer area between the two regions has to be determined with more accuracy.

According to the model and the experiment, one may assume that the overall heat transfer coefficient is higher than the known empirical and theoretical value. The overestimate of the heat transfer coefficient is primarily due to the difficulty in measuring the strand temperature since the helium and strands have large contact area between them. It is also important to understand the coupling time constant of the conductor since it reduces the large heat input in a short time period.

\subsection{Suggested Future Work}

It may be worthwhile to change the size of thermometer probe to see if there is any impact on the over all thermal hydraulic characteristics of the conductor. The installation of another flow meter at the inlet of conductor will provide more information on the flow reduction caused by the transient disturbance.

Understanding the transverse heat transfer mechanism is very important to characterize the thermal hydraulic effects in hybrid conductors. Therefore, extending the transient heat 
transfer experiment to include forced flow condition should be very interesting. However, it is essential to understand the temperature distribution in the bundle region along the conductor with the inductive heat input. This can be done by mounting a series of thermometers along the conductor. Thermometry has to be improved to obtain the strands temperature. This should require extensive study and experiment since modifications to the bundle region affects the overall hydraulic characteristics. 


\section{References}

1. M.N. Wilson, "Stabilization, Protection and Current Density: some general observations and speculations", Cryogenics, Vol. 31, 499 (1991).

2. D.L. Martin et al. “ Reaction Treatment, Critical Current, Transition Temperature and Bend Properties of a Niobium-Bronze Process Multifilamentary Superconductor", IEEE Trans. on Mag., Mag-15, 185 (1979).

3. S.W. Van Sciver, "Helium Cryogenics", Plenum Press (1986)

4. P.L. Kapitza, Nature, London, vol. 141, 74 (1933).

5. J.F. Allen and A.D. Misener, Nature, London, 141,75 (1938).

6. W.H. Keesom and A.P. Keesom, Physica, Vol. 3, 75 (1938).

7. V.P. Peshkov, Journ. Physics, Moscow, 10, 359 (1936)

8. L.Tisza, Physics. Rev. 72,838 (1947).

9. C.J. Gorter and J.H. Mellink, "On the Irreversible Processes in Liquid Helium II", Physica, 15 , 285 (1949)

10. L. Dresner, "Transient Heat Transfer in Superfluid Helium-Part II", Adv. Cryo. Engr., Vol. 29, 323 (1984).

11. G.L. Pollack, "Kapitza Resistance", Review of Modern Physics, 48 (1964).

12. K.M. Khalatvikov, Zh. Eks. Theor. Fiz. Vol. 22, 687 (1952).

13. W.B. Bald, "Supercritical Helium Cooling of Hollow Superconductors", Adv. Cryo. Eng., Vol. 16, 368 (1970).

14. V. Arp, "Forced Flow, Single Phase Helium Cooling Systems", Adv. Cryo. Eng., Vol. 17, 342 (1972).

15. J.W. Dean, W. Stewart and J.K. Hoffer, "Temperature Profiles in a Long Gaseous Helium Cooled Tube", Adv. Cryo. Eng., Vol. 23, 250 (1978. 
16. P.J. Giarratano and V.D. Arp and R.V. Smith, "Forced Convection Heat Transfer to Supercritical Helium", Cryogenics, 385 (1971).

17. P.J. Giarratano and W.G. Steward, "Transient Forced Convection Heat Transfer to Helium During a Step in Heat Flux", Trans. ASME, Joun. of Heat Transfer, Vol. 105, 350 (1983).

18. W.B. Bloem, "Transient Heat Transfer to a Forced Flow of Supercritical Helium at 4.2 K", Cryogenics, Vol. 26, 300 (1986).

19. J.W. Lue, J.R. Miller and L. Dresner, "Stability of Cable-in-Conduit Conductors", J. Appl. Physics. Vol. 51, 772 (1980).

20. N. Mitchell, "Conductor Design for the ITER Toroidal and Poloidal Magnetic Systems", IEEE Trans. Mag., Vol. 30, 1602 (1994).

21. P.E. Phelan et al., "Transient Stability of a Nb-Ti Cable-in-Conduit Superconductor: Experimental Results", Cryogenics, Vol. 29, 109 (1989).

22. J.C. Lottin and J.R. Miller, "Stability of Internally Cooled Superconductors in the Temperature Range 1.8 to 4.2 K", IEEE Trans. on Mag., Vol. Mag-19, 439 (1983).

23. K. Agatsuma, "Influence of Mass Flow Rate and Hydraulic Perimeter on the Transient Stability Margin of a Forced Flow Cooled Superconductor", Cryogenics, Vol. 31, 551 (1991).

24. J.W. Lue and J.R. Miller, "Parametric Study of the Stability Margins of Cable-inConduit Superconductors: Experiment", IEEE Trans. on Mag. Vol. Mag-17, 757 (1981).

25. L. Dresner, "Heating-induced Flows in Cable-in-Conduit Conductors", Cryogenics, 653 (1979).

26. L. Dresner, "Parametric Study of the Stability Margin of Cable-in-Conduit Superconductors: Theory", IEEE Trans. on Mag. Vol. Mag-17, 753 (1981). 
27. S.R. Shanfield et al., "Transient Cooling in Internally Cooled Cabled Superconductors (ICS)", IEEE Trans. on Mag., Vol. Mag-17, 2019 (1981).

28. V. Arp, "Transient Helium Hydrodynamics in Forced-Cooled Superconducting Cables", presented ar the Superconducting MHD Magnet Design Conference, (1980).

29. L. Bottura and J.V. Minervini, "Numerical Analysis of Heating Induced Transient Flow in Supercritical Helium", Adv. in Cryo. Eng.., Vol. 35, 395 (1990).

30. J.W. Lue, J.R. Miller and L. Dresner, "Stability of Cable-in-Conduit Conductors", J. Appl. Physics. Vol. 51, 772 (1980).

31. L. Dresner, "Stability of Cable-in-Conduit Force-Cooled Conductors: Elementary Theory", ORNL/TM-6657, (1979).

32. C. Schmidt, Applied Physics Letter, Vol. 32, 827 (1978).

33. V. Arp, "Forced Flow, Single-Phase Helium Cooling Systems", Adv. Cryo. Eng. Vol. $17,342(1972)$.

34. J.W. Lue, J.R. Miller and J.C. Lottin, "Pressure Drop Measurements on Forced Flow Cable Conductors", IEEE Trans. on Mag., Vol. Mag-15, 53 (1979).

35. Y. Takahashi et al., "Experimental Results of JF-15 Forced-Cooled Superconducting Test Loop", 10th Intl. Cryo. Eng.. Conf., Helsinki, 87 (1984).

36. S. Shimamoto et al., "Development of a Large Forced-Cooled Pulse Coil for the Next Tokamak", IEEE 1 lth Symp. on Fusion Eng., 1017 (1985).

37. M. Sugimoto et al. "Test Results of the DPC-TJ: Thermal and Hydraulic Performance", Cryogenics, Vol. 33, 597 (1993).

38. H. Katheder, "A General Formula for Calculation of the Friction Factor for Cable in Conduit Conductors", The NET team, Internal note, N/R/0821/26/A.

39. M.A. Daugherty, “Helium Hydrodynamics of Internally Cooled Cabled Superconductors“, Ph.D Thesis, University of Wisconsin-Madison, 1991. 
40. M.A. Daugherty, Y Huang and S.W. Van Sciver, "Pressure Drop Measurements on Supercritical Helium Cooled Cable in Conduit Conductors", IEEE Trans. on Mag., Mag-2, No. 2, 1512 (1989).

41. Takahata et al., "Experimental Results of the R\&D Forced-Flow Poloidal Coil (TokiPF)", presented at Fusion Eng.. and Design North-Holland, (1993).

42. A. Martinez and B. Turck, "Supercritical Helium Cooling of a Cable in Conduit Conductor with an Inner Tube", internal note at CEA, (1993)

43. L. Bottura, "Thermohydraulics of CICC's with Central Cooling Passage", presented at the Applied Superconductivity Conference in Boston, (1994).

44. J.G. Weisend II, Y. Huang and S.W. Van Sciver, "Testing of Two Heat Exchangers in He I", ASME 7th Inter. Cryo. Sympo. (1989)

45. R.L. Rusby, “New Vapor Pressure Equations for ${ }^{4} \mathrm{He}$ and ${ }^{3} \mathrm{He}$ ”, Journ. of Low Temp. Physics, Vol. 58, 203 (1985).

46. F.S. Shih, "Laminar Flow in Axisymetric Conduit by a Rational Approach", The Canadian Journal of Chemical Engineering, Vol. 45.285 (1967).

47. D.J. Gunn and C.W. Darling, "Fluid Flow and Energy Loss in Non-Circular Conduits", Trans. Instn. Chem. Eng., Vol. 41, 12 (1963).

48. S.W. Van Sciver et al. "AC Loss Measurement of the SMES/CIC Conductor", Cryogenics, Vol. 34, 615 (1994).

49. S. Uchida, "The Pulsating Viscous Flow Superposed on the Steady Laminar Motion of Incompressible Fluid in a Circular Pipe", ZAMP 7, 403-422 (1950). 